\title{
Abstracts of 17th World Congress of the International Federation of Colposcopy and Cervical Pathology-IFCPC 2021
}

(C) Association of Gynecologic Oncologists of India 2021

\section{1: Basic Research in HPV and Cervical Cancer}

\section{Genomics of HPV Associated Disease}

\section{Abstract \#128: Epidemiology of Human Papillomavirus in Women Older than 30 Years}

Shubham Bidhuri, Saritha Shamsunder

Department of Obstetrics and Gynaecology, Vardhaman Mahavir Medical College \& Safdarjung Hospital, New Delhi, India

Objectives: To study the prevalence of HPV and its various strains in women more than 30 years of age in our population.

Methods: This observational study was carried out in a tertiary care hospital where women older than 30 years of age attending the gynecology outpatient department were enrolled. Cervical samples were sent for HPV DNA testing in a total of 1757 women, out of which 150 were positive for HPV. Positive women were further subjected to Pap smear test with colposcopy and directed biopsy if required and were further managed according to histopathology reports.

Results: The prevalence of HPV was $8.53 \%$. Among 150 HPV-positive women, HPV 16 was the most common type with prevalence of $54.1 \%$, followed by HPV 33 and 52 with prevalence of $8.78 \%$ and $7.8 \%$, respectively. Other HPV types found were HPV $18(6.8 \%)$, HPV 6 (4.8\%), HPV 45 (4.8\%) HPV 58 (4.8\%), HPV 39 (2.93\%), HPV $11(2.93 \%)$ and HPV $31(1.95 \%)$. The majority of women had infection with single type of HPV (70.67\%), while $29.33 \%$ women harboured infection with more than one type of HPV. The most common combination was found to be with HPV 16 and 33 (13.6\%). Conclusions: The prevalence of HPV infection found in women older than 30 years of age was $8.53 \%$. HPV 16 was the most common genotype. followed by HPV 33, 52 and 18 in decreasing order of prevalence. Phylogenetically related HPV tend to occur together in infection with more than one type of HPV strains.
Virus-Host interaction

Abstract \#83: Role of host cervico-vaginal microbiome in persistence of high-risk HPV infection

Shakuntala P N, Renuka Ramaiah

ESIC PGIMSR\& Medical College, Rajajinagar, Bangalore, Karnataka, India

\section{Objectives:}

1. To identify women with hrHPV (high-risk human papillomavirus) by using HYBRID CAPTURE 2 HPV DNA test.

2. To study the patterns and association of cervico-vaginal microbiome in the development of hrHPV infection.

Methods: Women underwent HYBRID CAPTURE 2 HPV DNA testing using BD SUREPATH. Then endocervical or vaginal swabs were obtained in all women to study the microbiome of the cervicovaginal area which were subjected to aerobic culture, candidal typing and lactobacilli colony count.

HrHPV-positive women were subjected to VIA/VILI and or colposcopy. If the modified Reid score was more than 4 , the cervix was biopsied. Histopathology was recorded. They were followed up and a repeat HYBRID CAPTURE 2 HPV DNA testing was done after 12 months.

Results: Fifty-three (13.25\%) were positive for high-risk HPV DNA testing out of 400 women tested. $20(37.75 \%)$ had repeat positivity for high-risk HPV DNA virus after 12 months. The microbiome pattern was found to be candidal infection being the commonest, followed by trichomoniasis in $5(9.43 \%)$ and E. coli in 4 (7.54\%). High-risk HPV DNA women had no or lower colony count which ranged from 0 to $1000 \mathrm{cfu} / \mathrm{ml}$.

Conclusions: Persistence of HPV was found in women who had low lactobacilli counts and associated recurrent candidal infections. However, parasitic infections like trichomoniasis did contribute, but in a lesser number of high-risk HPV-positive women. 
Abstract \#317: Sexual Risk Behaviors and Pattern of Multiple Sites Concordance of Human Papillomavirus Infections Among Sexually Active Nigerian Women

Imran O. Morhason-Bello ${ }^{1,2}$, Kathy Baisley ${ }^{3}$, Isaac F. Adewole ${ }^{1}$, Miquel Pavon ${ }^{4}$, Rasheed Bakare ${ }^{5}$, Silvia de Sanjosé ${ }^{4}$ Suzanna Francis $^{3}$, Deborah Watson-Jones ${ }^{6,7}$

\section{Affiliations}

1. Obstetrics and Gynaecology Department, Faculty of Clinical Sciences, College of Medicine, University of Ibadan, Ibadan, Nigeria.

2. Institute of Advance Medical Research and Training, College of Medicine, University of Ibadan, Ibadan, Nigeria

3. Department of Infectious Disease Epidemiology, Faculty of Epidemiology and Population Health, London School of Hygiene and Tropical Medicine, Keppel St, London WC1E 7HT United Kingdom

4. Institut Catalàd'Oncologia / Catalan Institute of Oncology, Cancer Epidemiology Research Program, Infections and Cancer Laboratory I Granvia de L'Hospitalet 199-203, 08908 L'Hospitalet de Llobregat, Spain I 932607123

5. Department of Microbiology, Faculty of Basic Medical Sciences, College of Medicine, University of Ibadan, Nigeria

6. Mwanza Intervention Trials Unit, National Institute for Medical Research, Mwanza, Tanzania

7. Clinical Research Department, Faculty of Infectious and Tropical Diseases, London School of Hygiene and Tropical Medicine, Keppel St, London WC1E 7HT, United Kingdom.

Background/Objective: Human papillomavirus (HPV) is primarily transmitted by unprotected sexual behaviors, and persistence of this virus is associated with HPV-related cancers of the cervix, anus, vulvar, oral cavity, and penis. Nigeria has one of the highest burdens of cervical cancer, but there is a paucity of data on other HPV-related cancers. This study was done to determine the prevalence of sexual risk behaviors and pattern of multiple sites and concordance of HPV infections among women aged 18-45 years in Ibadan, Nigeria.

Methods: The study was conducted as part of Sexual behavior and HPV Infections in Nigerians in Ibadan (SHINI). Randomly selected healthy sexually active women aged 18-45 years from two communities (peri-urban and urban) in Ibadan had face-to-face interviews including sexual behaviours and clinical examinations. A gendermatched nurse collected samples from the mouth, cervix, vulvar and anal sites and stored at $-80{ }^{\circ} \mathrm{C}$ prior to HPV genotyping at the Catalan Institute of Oncology, Spain, by the Anyplex ${ }^{\mathrm{TM}}$ II HPV28 assay. Analysis was performed with STATA 15 software.

Results: The results of 310 women enrolled were analyzed. The mean age was $29.0 \pm 7.0$ years. $147 / 310(47 \%)$ had multiple penile-vaginal sex partners, $35 / 310(11 \%)$ had ever given oral sex, while $36 / 310$ $(12 \%)$ had ever received oral sex in heterosexual relationship, and only 1 participant reported history of penile-anal sex. The prevalence of any HPV infection in the vulvar, cervical, anal and oral sites were $68 \%, 60 \%, 57 \%$ and $16 \%$, respectively. Any high-risk HPV infections was highest in the vulvar (51\%), followed by the cervix (44\%), anal $(39 \%)$, and oral $(11 \%)$ sites. A similar pattern was observed in the prevalence of multiple HPV genotypes infections $(\geq 2)$ across the sites - vulvar $(41 \%)$, cervix (33\%), anal cavity (34\%), and oral site (5\%). The concordance of any HPV, any high-risk HPV and any lowrisk HPV in the four anatomic sites were $31 / 310(10 \%), 19 / 310(6 \%)$ and 16/310 (5\%), respectively. The high-risk and low-risk HPV genotypes with the commonest concordance in the four anatomic sites were HPV-16/18 and HPV-42, respectively.

Conclusion: Sexual risk behaviors and HPV infections are common among sexually active Nigerian women. There is also demonstrable multiple sites concordance of HPV infections in these women. Further longitudinal studies are being planned to explore the incidence and clearance rates of multiple sites HPV infections in Nigeria.

Acknowledgements: IMB received grant support from the University of Ibadan, Ibadan, Nigeria for his $\mathrm{PhD}$ programme at the London School of Hygiene and Tropical Medicine, London, UK, and the conduct of the SHINI study in Nigeria.

\section{Transformation and Carcinogenesis and Other Basic Research}

Abstract \#142: The different expression of estrogen receptor in epithelial and stroma in different cervical lesions

Mingzhu Li, Chao Zhao, Yun Zhao, Jingran Li, Wei Lihui

Peking University People's Hospital, China

Objectives: The aim of this study was to investigate the expression of estrogen receptor (ER) in the epithelial and stroma tissues of normal (NILM), cervical intraepithelial neoplasia (CIN), and cervical cancer, as well as their relationship to clinical characteristics and the status of human papillomavirus (HPV) infection.

Methods: From 2008 to 2014, 86 cases from People's Hospital of Peking University were retrospectively analyzed, including 21 cases of normal pathological diagnosis, 9 cases of LSIL, 15 cases of HSIL, 36 cases of squamous cell carcinoma, and 5 cases of adenocarcinoma. Results: The median age of the patients with high ER stroma expression was $50.8 \pm 12.4$ years, and $45.7 \pm 8.8$ years in low or no expression group, the difference was statistically significant $(\mathrm{P}<0.05)$. In paraffin specimens, the epithelial expression of ER in CIN was significantly lower than that in NILM, and decreased to undetectable level in carcinoma $(\mathrm{P}<0.05)$. However, stroma expression of ER was expressed continuously in different cervical lesions. Among those with high expression of ER in epithelium, 77.8 (7/9) also showed high expression in the cervical stroma. Those with no ER stroma expression were also absent in the epithelium (14/14). Among the patients with no ER expression in epithelium, 64.5\% (20/ 31 ) had high ER expression in stroma, and $80 \%(16 / 20)$ of them were high-grade lesion and above (HSIL+). Clinicopathological characteristics of cervical cancer and the status of HPV infection all showed no correlation with stroma expression of $\mathrm{ER} \alpha(\mathrm{P}>0.05)$.

Conclusions: Stromal ER expression was independent of HPV infection and irrespective of the degree of cervical lesion and epithelium ER expression.

Abstract \#144: Role of cervical cancer biomarkers p16 \& Ki67 in abnormal cervical cytology smear

Mayank Jain, Meenakshi Gothwal, Pratibha Singh, Garima Yadav, Navdeep Kaur Ghuman, Aasma Nalwa

Department of Obstetrics and Gynaecology, All India Institute of Medical Sciences, Jodhpur, India

Objectives: To study the efficacy of immunostaining with p16/ Ki-67 in predicting the presence of significant lesion in cases of mild cytological atypia.

Methods: It was a prospective cross-sectional study for a duration of 12 months held at AIIMS, Jodhpur. Liquid-based cytology was done in patients $>21 \mathrm{yrs}$ of age attending the gynecology OPD. Smears with abnormal findings underwent Immunostaining for p16/Ki-67. Screen positive females were managed by the standard protocol of colposcopy-guided cervical biopsy, and thus histopathological correlation with cervical biopsy was done. 
Results: A total of 2134 LBC were done, out of which 46 cases had abnormal cytological findings. Out of these 46 cases, 38 smears underwent Immunostaining for p16/Ki-67.

Twenty-eight cases had shown co-staining for both p16 and Ki-67, suggestive of true HPV infection of the cells. In the ASC-US group, the sensitivity and specificity of the immunostaining in diagnosing CIN2 + lesions were $87.51 \%$ and in LSIL group, the sensitivity and specificity of the immunostaining in diagnosing CIN2 + lesions were $100 \%$. p16/Ki-67 positivity also increased with cytological severity which in turn corresponded with histological findings: It reached from $50 \%$ in ASC-US to $100 \%$ in both HSIL and SCC categories.

Conclusions: Immunostaining with p16 \& Ki67 can be a useful method in the triage of the ASC-US and the LSIL group as considering the high sensitivity and specificity value.

\section{Abstract \#169: Role of Host Cervico-vaginal Microbiome in Persistence of High-Risk HPV Infection in Women Attending Gynecology Outpatient Department in a Tertiary Hospital}

Shakuntala P N, Renuka Ramaiah, Rudresh SM

ESIC MC\& PGI MSR, Rajajinagar, Bangalore, India

Objectives: The objective of the study was to identify women with hrHPV (high-risk human papillomavirus) by using HYBRID CAPTURE II HPV DNA test and to study the patterns and association of cervico-vaginal microbiome in the development of hrHPV infection. Design: Descriptive analytical, prospective study

Methods: Women underwent HYBRID CAPTURE II HPV DNA testing using BD SUREPATH. Then endocervical or vaginal swabs were obtained in all women to study the microbiome of the cervicovaginal area which were subjected to aerobic culture, candida typing and lactobacilli colony count. hrHPV-positive women were subjected to VIA/VILI and or colposcopy. If the modified Reid score was more than 4, the cervix was biopsied. Histopathology was recorded. They were followed up and a repeat HYBRID CAPTURE II HPV DNA testing was done after 12 months.

Results: Fifty-three (13.25\%) were positive for high-risk HPV DNA testing out of 400 women tested. $20(37.75 \%)$ had repeat positivity for high-risk HPV DNA virus after 12 months. The microbiome pattern was found to be candida infection being the commonest, followed by trichomoniasis in $5(9.43 \%)$ and E. coli in 4 (7.54\%). High-risk HPV DNA women had no or lower colony count which ranged from 0 to $1000 \mathrm{cfu} / \mathrm{ml} .(32.5 \%)$

Conclusions: Persistence of HPV was found in women who had low lactobacilli counts and associated recurrent candida infections. However, parasitic infections like trichomoniasis did contribute, but in a lesser number of high-risk HPV-positive women.

\section{Abstract \#179: Expression of Calcium Activated Potassium Channels (KCNMA1) in Cervical Tissue as a Biomarker of Cervical Pre-cancer and Cancer: a Comparative Study}

Puja Kumari ${ }^{1}$, Bindiya Gupta ${ }^{1}$, Shalini Rajaram ${ }^{1}$, Priyanka Gogoi ${ }^{2}$, Rajarshi $\mathrm{Kar}^{3}$, Sandhya Jain ${ }^{1}$

${ }^{1}$ Department of Obstetrics and Gynecology,

${ }^{2}$ Department of Pathology,

${ }^{3}$ Department of Biochemistry UCMS \& GTB Hospital, New Delhi, India

Background and Objectives: Cervical cancer is the major cause of mortality in developing countries. Ion channels have shown promising result as a biomarker of cancers. KCNMA1 channel expression in cervical biopsies has shown differential expression, while non- cancerous cervix did not show any KCNMA1 protein expression, most of the tissues with cervical lesions displayed the presence of protein. The objective was to compare and correlate the mRNA and protein expression of KCNMA1 channels in normal, pre-invasive and invasive cervical cancer.

Methods: A total of 201 screen-positive women (VIA, VILI and Pap test) underwent colposcopy. Cervical biopsies were taken and stored for estimation KCNMA1 mRNA levels and protein expression. Women with Swede score of 4 or more $(n=92)$ were biopsied. Cervical biopsy taken in women with obvious cervical growth $(n=8)$, without prior colposcopy. For controls, 15 women were included in the study who underwent hysterectomy for benign gynecological indication with normal screening tests. From the above cohort, 60 women were recruited to the study and allocated equally $(n=15)$ into four groups based on histopathology, i.e., control (Group1), CIN1 (Group2), CIN 2/3 (Group 3) and invasive cervical carcinoma (Group 4).

Result: KCNMA1 mRNA level estimation was done by real-time RT-PCR. The mean KCNMA1 mRNA levels in Control (group1) were 0.2253 ( $\mathrm{SD} \pm 0.5798)$, while the mean mRNA level in CIN1 (group 2) was 271.40 (SD \pm 1050.21 ), mean mRNA level in CIN2/3 (group 3) was 298.84 (SD \pm 1153.33 ) and mean KCNMA1 mRNA level in cancer cervix (group4) was 326.545 (SD \pm 861.97). KCNMA1 protein expression was done by immunohistochemistry using anti-KCNMA1 rabbit polyclonal antibody against Maxi Potassium channel alpha. In the control group, all had negative KCNMA1 channel protein expression, while positive expression was seen in all cases $(100 \%)$ in the cancer cervix group. In CIN1, 34\% $(n=5)$ had positive expression, while $66 \%(\mathrm{n}=10)$ had negative expression. In CIN2/3, 80\% $(n=12)$ had positive expression and $20 \%(n=3)$ had negative expression.

Conclusions: KCNMA1 channel expression has a promising role as a biomarker of cervical precancer and cancer.

\section{Abstract \#181: The Role of Human Papillomavirus (HPV) In The Development Of Cervical Carcinoma And Its Early Detection By Molecular Method}

Sangeeta Pankaj, Jyotsna Rani, Kavya Abhilashi, Pratibha Kumari, Satya Kumari, Vijayanand Choudhary, Nidhi Prasad, Babban Jee

State Cancer Institute, Indira Gandhi Institute of Medical Sciences, Patna, Bihar, India

Objectives: Primary HPV DNA testing is now in wide practice replacing cytology in many countries. Fundamental information about the development and progression of HPV-associated cervical carcinogenesis is based on interaction of host proteins with HPV E6 and E7 oncoproteins. The aim of the present study is to evaluate the efficacy of E6/E7 mRNA assay (Aptima test) as alternatives or adjunctive test to cytology in the future.

Methods: A total of 106 women attending OPD of Gynecological Oncology, SCI at IGIMS, Patna, were recruited in this prospective study and underwent molecular tests (HPV DNA test and Aptima test for mRNA assay), cytological test, and colposcopy-guided biopsy. Performance of the molecular tests and cytology as screening test were evaluated considering histopathological diagnosis as gold-standard and confirmatory one, and the collected data were analysed statistically.

Results: A total of 56 out of 106 participants had abnormal screening results in the form of positive HPV DNA or E6/E7 mRNA with or without abnormal cytology or had histopathologically confirmed premalignant/malignant lesion.47.16\%(50/106) and 32\% (34/106) women were positive for HPV DNA and E6/E7 mRNA assay, respectively. 33\% (35/106) women had abnormal cytology and $39.6 \%$ (42/106) had histologically confirmed CIN and invasive cancer. Sensitivity, specificity, PPV \& NPV of cytology, and HPV DNA were 
calculated as $83.3 \%, 97.2 \%, 92.6 \%$ and $93.4 \%$ versus $83.8 \%, 68 \%$, $52 \%$ and $91 \%$, respectively. Aptima test was found more specific $(93.5 \%)$ and sensitive (95.5\%) with PPV \& NPV as $85.2 \%$ and $97.2 \%$, respectively.

Conclusions: Based on the findings of index study, molecular tests are found equally good in performance with advantage of being more objective than cytology. E6/E7 mRNA assay can be recommended as an adjunct to HPV DNA test or cytology for the purpose of cervical cancer screening at mass level and may be helpful in triaging to reduce the load for colposcopy.

\section{Abstract \#217: Role of P16 Ink4A, Ki-67 \& L1 Capsid Protein in Cervical Biopsy}

Usha Sarma $^{1}$, Gokul Ch Das ${ }^{2}$, Nirnay Sarma ${ }^{3}$

Department of Pathology ${ }^{1}$, Department of Obstetrics and Gynaecology $^{2}$, Guwahati Medical College hospital. Guwahati

${ }^{3}$ MBBS student, All India Institute of Medical Science, Guwahati, India

Objectives: To analyse the immunohistochemical expression of p16, HPV L1 capsid protein and Ki67 in CIN and cervical cancer, and their utility to determine the accuracy of histological diagnosis and prediction of biological behaviour of cervical lesion

Design: A retrospective cross-sectional study was carried out. The data and the tissue blocks were taken from archive of pathology Department, Gauhati Medical College, Guwahati.

Methods: In 110 numbers of cervical biopsy included 25 CIN1, 21 CIN2, 12 CIN3, 26 SCC and 01 adenocarcinoma and 25 non-neoplastic lesion. The tissue sections were stained withP ${ }^{16 I N K 4 a}, \mathrm{Ki}-67 \mathrm{~L} 1$ capsid protein.

Results: $\mathrm{P} 16^{\mathrm{INK} 4 \mathrm{a}}$ was expressed in $51.8 \%$ (1.8\% in non-dysplasia, and $50 \%$ in CIN of varying grade and invasive cancer). Ki-67 was expressed in $55.5 \%$ cases (3.6\% non-dysplasia and 51.8\% dysplasia / CIN of varying grade and invasive cancer). There was high correlation between the two variables P $16+$ ve $\& \mathrm{Ki}-67+$ ve cases with their histologic grade. L1 capsid protein is detected in $10.9 \%$ cases, out of which $8.2 \%$ CIN1 and $2.7 \%$ CIN2. It is not found in high-grade lesion. A significant association is observed between L1 capsid protein and the histologic grading $\left(\chi^{2}=24.77^{*}, \mathrm{p}<0.0005\right)$

Conclusion: Though histopathology remains the "gold standard" for the diagnosis of CIN, both low and high-grade, biomarkers like P16 ${ }^{\mathrm{INK} 4 \mathrm{a}}$, Ki-67 and L1 Capsid protein have emerged as helpful adjuncts. Their combined use may assist in the histopathologic classification of pre-invasive lesions and determines the behaviour of the cervical lesion.

\section{Abstract \#219: Role of Cervical Cancer Biomarkers P16 \& Ki67 in Abnormal Cervical Cytological Smear: A Study from Tertiary Care Center of Western Rajasthan}

Meenakshi Gothwal, Pratibha Singh, Aasma Nalwa, Garima Yadav, Charu Sharma Department of Obstetrics \& Gynecology, All India Institute of Medical Sciences, Jodhpur, Rajasthan

Introduction: Cervical cancer is the most common cancer in India. Screening of cervical cancer helps in marked reduction in invasive cervical cancers. The low sensitivity of Papanicolaou cytology (Pap smear) and high-risk human papillomavirus (HR-HPV) in excluding high-grade intraepithelial lesion (ASC-H) leads to unnecessary referrals to colposcopy-guided biopsy. The combined cervical cytology screening and HR-HPV has its own limitations and still need further standardization. Using additional biomarkers like staining with p16 and Ki-67 might help in triaging abnormal pap smear.
Objective: To know the efficacy of immunostaining with p16/Ki-67 in predicting the presence of significant lesion in cases of mild cytological atypia

Methods: A prospective, cross-sectional study. Pap smears conventional and LBC along with P16, Ki-67 and available biopsies were correlated.

Results: Liquid base cytology (LBC) was done in 2134 cases, out of which 46 cases showed abnormal cytological findings. Immunostaining with p16 and Ki-67 was performed on 38 cases of abnormal cytological smears. Out of 38 abnormal cytology cases, 28 cases had shown co-staining for both p16 and Ki-67. Of the 38 cases, 07/14 ASCUS, 06/06 HSIL, 07/08 ASC-H, 05/05 Squamous cell carcinoma and 02/02 Adenocarcinoma also showed dual positivity for p16 and $\mathrm{Ki}-67$. In the ASC-US group, the sensitivity and specificity of the immunostaining in diagnosing CIN2 + lesions were $87.51 \%$ and in LSIL group, the sensitivity and specificity of the immunostaining in diagnosing CIN2 + lesions were $100 \%$.

Conclusion: This immunostaining with p16 \& Ki67 can be a useful method in the triaging of the ASC-US and the LSIL group as considering the high sensitivity and specificity values.

\section{Abstract \#260: The Relationship Between Serum Vitamin D Levels and Cervical Intraepithelial Neoplasia}

Michael C. Ezeanochie ${ }^{1}$, Olasimbo Peter ${ }^{1}$. Anthony Okonkwo ${ }^{1}$

${ }^{1}$ Department of Obstetrics and Gynecology, University of Benin Teaching Hospital, Benin City, Edo State, Nigeria

Objective: Vitamin D administration in women with cervical intraepithelial neoplasia (CIN) 1 has resulted in its regression. This study compared the serum levels of Vitamin D among women diagnosed with CIN to those without CIN to determine if differences exist at the time of diagnosis.

Design: A matched case-control study in 2019 at the University of Benin Teaching Hospital, Nigeria.

Method: The cases comprised 30 women with CIN while the controls were 30 women with normal Pap smear results. Vitamin D levels, sociodemographic and clinical details were compared between both groups and with CIN 1. CIN 2 and CIN 3, respectively. Statistical analysis was with SPSS version 22. Differences in mean values were compared with the $t$ test and ANOVA test, while categorical variables were compared with Chi-square or Fishers exact test. Statistical significance was at $\mathrm{p}<0.05$.

Results: There was no significant difference in the sociodemographic details of both groups. There was no significant different in the mean serum Vit D levels between the cases and the controls $(94 \pm 82.81$ versus $88.62 \pm 53.72 \mathrm{ng} / \mathrm{ml}$, respectively, $\mathrm{p}=.67$ ). Vit $\mathrm{D}$ level in women with normal cervix compared to subgroups of CIN 1, CIN 2, and CIN 3 did not reveal any significant difference $(94.00 \pm 82.81$ versus $\quad 90.15 \pm 56.34$ versus $103.34 \pm 49.96$ versus $63.82 \pm 41.03 \mathrm{ng} / \mathrm{ml}$ respectively, $\mathrm{p}=.61$ )

Conclusions: There is no significant difference in the mean serum Vitamin D levels in women without CIN and those with CIN. Further studies are required to explore the relationship between it and CIN in the control of cervical cancer.

\author{
Abstract \#276: ROC-analysis derived Immunohistochemical p53 \\ cut-off scores for diagnostic compartmentalization of cervical \\ lesions \\ Ayushi Shukla ${ }^{1}$, Rekha Sachan ${ }^{1}$, Malti Maurya ${ }^{2}$, Uma Singh $^{1}$ \\ ${ }^{1}$ Department of Obstetrics and Gynecology, ${ }^{2}$ Department of \\ Pathology, King George's Medical University, Lucknow, India
}


Objective: To compare IHC (immunohistochemical) p53 expression in spectrum of cervical lesions: CC (Chronic cervicitis), CIN (Cervical Intraepithelial Neoplasia) and SCC (Squamous Cell Carcinoma of Cervix) and propose p53 cut-off scores using ROC analysis for better discrimination of these groups.

Method: Prospective study where immunohistochemical analysis of biomarker p53 was performed on 100 cervical histology samples: 15 CC serving as control, $40 \mathrm{CIN}$ and 45 SCC obtained from biopsy (colposcopy aided where indicated) of symptomatic women attending gynecological outpatient department. Nuclear immunoreactivity was assessed using semi-quantitative scoring system. Mean p53 score in each group was compared using Kruskal-Wallis test. ROC curves, AUC, sensitivity, and specificity were calculated to obtain cut-off scores best differentiating the three groups.

Results: Mean p53 score showed graded increment with increasing severity of lesion $(\mathrm{p}<0.001)$. Selected cut-off scores reflected good discriminating power between non-malignant (CC) and premalignant lesions (CIN) (AUC-0.714, Specificity-100\%) and between premalignant (CIN) and malignant (SCC) lesions (AUC-0.781, specificity$88.9 \%$ ). Intra-CIN differentiation was reasonably achieved with following parameters: CIN 1 vs 2 (AUC-0.621, specificity-85.7\%) and CIN 2 vs 3 (AUC-0.615, specificity-100\%). Proposed p53 scoring and immunoscore cut-offs model has an overall diagnostic accuracy of $61 \%$ compared to previous arbitrary p53 IHC interpretations cut-offs ( $>10 \%$ positive nuclei or immunoscore cut-off 2 ).

Conclusion: p53 expression can be utilized as a clinically useful biomarker in differentiating cervical non-malignant, premalignant, and malignant disease and aiding histopathology in a better diagnostic categorization of CIN group. ROC-derived cut-off scores can provide much needed objectivity and optimal decision thresholds to IHC interpretation.

\section{Abstract \#296: Circulating HPV DNA as a biomarker in patients with HPV-related cervical carcinoma}

S Bryan, J Lee, R Gunu, A Jones, A Olaitan, A Rosenthal, S Bhide

University College London Hospital, UK

Objectives: In persistent HPV infections that lead to cancer, the tumor breaches the basement membrane releasing HPV DNA into the bloodstream. A next-generation sequencing (NGS) assay for detection of plasma HPV cDNA has been developed and demonstrates $88 \%$ sensitivity and $100 \%$ specificity in patients with locally advanced cervix cancers undergoing radical chemoradiation. We investigated this expanded panel assay in pre-invasive and invasive cervical lesions. We hypothesize HPV cDNA is detectable in invasive cancers but not pre-invasive lesions.

Methods: Two cohorts were recruited: those undergoing treatment of high-grade CIN and those with early invasive cervix cancer (1A-1B). A blood sample was taken immediately prior to treatment and again at follow-up. DNA extraction followed by NGS was used for detection of HPV cDNA.

Results: We recruited 52 patients: 40 with high-grade lesions and 12 with invasive tumors. Sequencing was performed on 50 baseline samples. None of the patients with pre-invasive lesions were positive for HPV cDNA. Two of the invasive samples were excluded due to high stage. Of the remaining 10 invasive tumors, 1 reached the threshold of positivity for HPV cDNA. Recalculating the thresholds for positivity did not increase the detection of HPV cDNA in plasma in early stage $(<=1 \mathrm{~B})$ tumors.

Conclusion: HPV cDNA is absent in high-grade CIN. In early cervical tumours, there was very low detection of HPV cDNA, using even the most sensitive of currently available technologies, i.e., NGS.
More sensitive assays are required before HPV cDNA can be used as a biomarker in this setting.

\section{Abstract \#300: The effect of demethylation on proliferation and signaling pathways of cervical cancer cells}

Mingzhu Li, Chao Zhao, Yun Zhao, Jingran Li, Wei Lihui

Peking University People's Hospital, Xicheng District, Beijing, P.R. China

Objective After treatment with 5-Aza-CdR, the proliferation of Hela, Siha, C33A, HPV16, HPV18E6 mRNA levels of cervical cancer cell lines and the expression of $\mathrm{p} 21$ and $\mathrm{p} 53$ proteins were observed. Methods After intervention with different concentrations of 5-AzaCdR in cervical cancer Hela, Siha and C33A cell lines for 7 days, the proliferation changes of the three kinds of cells were detected by CCK8. Changes in HPV16 andHPV18 E6 expression were detected by RT-PCR. Protein expression of p21 and p53 was detected by Western blot.

Results The methylation level of PAX1 was expressed from high to low in Siha, Hela and C33A, respectively, while the methylase level of the three cell lines was on the rise vice versa, with statistically significant differences $(\mathrm{P}<0.05)$. With the increase in 5-Aza-CdR concentration, the proliferation inhibition of Hela, Siha and C33A cells increased, showing a dose-dependent relationship. After demethylation, the levels of HPV16E6 in Siha cells and HPV18 E6 in Hela cells showed a downward trend, while the levels of TERT mRNA in Hela, Siha and C33A cells all showed a downward trend. After demethylation, p53 and p21 protein contents in Siha, Hela and C33A cells of cervical cancer were significantly increased.

Conclusion: There were differences in the levels of PAX1 methylation and related methylase in different cervical cancer cell lines with different HPV states. Methylation inhibitors inhibit TERT mRNA levels by activating p53 and p21 signaling pathways, thereby inhibiting HPVE6 levels and inhibiting cervical cancer cell growth.

\section{Abstract \#321: To study the expression of epithelial-mesenchymal transition markers (E-cadherin and vimentin) in premalignant and malignant lesions of cervix}

Purnima Singh ${ }^{1}$, Sunita Malik ${ }^{1}$, Charanjeet Ahluwalia ${ }^{2}$

${ }^{1}$ Department of Obstetrics and Gynecology, ${ }^{2}$ Department of Pathology. VMMC and Safdarjung hospital, New Delhi, India

Objective: Epithelial-mesenchymal transition (EMT) is accompanied by changes in expression of differentiation markers from epithelial to mesenchymal. There is an increase in mesenchymal markers (vimentin) and decrease in epithelial markers (E-cadherin) as the cancer progresses from preinvasive to invasive state.

To study the expression of EMT markers (E-cadherin and vimentin) in premalignant and malignant lesions of cervix.

Design: Observational cross-sectional study.

Methods: Women attending gynecology OPD with various complaints were screened by Pap smear/VIA and recruited if found screen positive or if obvious growth is seen. Colposcopy-directed biopsy was done in these cases and those who were positive for cervical intraepithelial neoplasia (CIN) and Cancer (CA) cervix on histopathology examination underwent immunohistochemical (IHC) staining for EMT markers.

Results: Out of total 600 women screened, 54 were biopsy-proven for $\mathrm{CIN}$ and carcinoma cervix. Among these E-cadherin was found to be positive in $9(41 \%) \mathrm{CIN}$ and $2(6.2 \%) \mathrm{CA}$ cervix, whereas vimentin 
was found to be positive in $1(4.5 \%) \mathrm{CIN}$ and 10 (31.2\%) CA cervix which is statistically significant.

Conclusions: As the grade of CIN increases E-cadherin positivity increases and vimentin decreases, whereas in staging of CA cervix, as the stage of cancer increases vimentin positivity increases and E-cadherin decreases.

\section{HPV Testing and HPV Vaccination}

\section{HPV testing}

\section{Abstract \#17: Performance of Molecular Hybrid capture 2 test in presence of co-infection with common Reproductive tract infections}

Kavita Anand, Sharmila Pimple, Gauravi Mishra, Surendra Shastri, Atanu Bhattacharjee

Tata Memorial Hospital, Mumbai, India

Objectives: Molecular Hybrid Capture 2 test $(\mathrm{HC} 2)$ is an accepted screening modality for cervical cancer in developed countries as the test demonstrates a good sensitivity (average 96\%) to detect highgrade cervical intraepithelial neoplasms (CIN2 + lesions). Presently, the issue with the HPVDNA HC2 test in implementing as a primary screening test in developing countries is its low sensitivity reported (Average $80 \%$ ) for diagnosing CIN2 + lesions. The multicentric study from India reported the $\mathrm{HC} 2$ sensitivity of $68.2 \%$ which was substantially lower as compared to developed countries. India has a huge burden of untreated reproductive tract infections (RTIs). The aim of the present study was to study whether the presence of clinical cervicitis /or co-infections with laboratory-diagnosed RTIs interfere with the test result of HPV DNA by HC2 method.

Methods: This is a case-control study. A total of 508 women, 254 women with clinical cervicitis and 254 asymptomatic women without cervicitis were enrolled as cases and control, respectively. A baseline cervicovaginal swabs for RTIs and $\mathrm{HC} 2$ test samples were collected for all women enrolled in the study. All women in the case arm received syndromic treatment for cervicitis. A repeat cervicovaginal swabs and HC2 test was collected for all women enrolled in the study after 7-14 days.

Results and Conclusion: The study demonstrated that the overall detection rates of HPV to improved by $\mathrm{HC} 2$ test by $4.5 \%$ among women treated for mucopurulent cervicitis. On controlling the biological and behaviour determinants by modelling, the study demonstrated influencing role of mucopurulent discharge associated with cervicitis on the test results of $\mathrm{HC} 2$ test.

\section{Abstract \#67: Effect of Intervention on Awareness of HPV Vaccination and Its Acceptance Among Postpartum Women}

Jyothi G S, Priyanka Sinha

Ramaiah Medical College and Hospitals, Bengaluru, India

Objectives: To assess the changes in awareness levels regarding HPV vaccination following intervention among postpartum women attending maternal health services and to assess the acceptance levels of HPV vaccination among the study subjects and enumerate reasons for non-vaccination.

Methods: All women nearing to term pregnancy, not received HPV vaccination, coming for ANC check-ups to the OPDs of Ramaiah Hospitals, Bengaluru, were given pretest to assess the knowledge of HPV vaccination followed by counseling. Post-tests were given postdelivery. Participants willing for HPV vaccination were given the first dose before discharge and called for subsequent doses during their post-natal visits.

Results: In the baseline survey, $38.7 \%$ were aware of cervical cancer. $20.68 \%$ knew that HPV was the main cause of cervical cancer. $5.33 \%$ were aware of HPV vaccination. After the health education, $93.33 \%$ became aware of cervical cancer and $97.33 \%$ of HPV vaccination. 90\% came to know that HPV was the main cause of cervical cancer. After educational intervention, $70 \%$ received first-dose HPV vaccination before discharge. $86.63 \%$ women completed all three doses and felt that it was worthwhile to receive the vaccine during postpartum. Among 30\% who were not willing for vaccination; $46.66 \%$ cited high cost and $22.22 \%$ were not allowed by the family members. Conclusion: Health education played a major role in increasing the awareness of cervical cancer and HPV vaccination. Recommendations from the physician and healthcare personals also showed high levels of acceptance for the HPV vaccination in postpartum women

\section{Abstract \#68: High-Risk Human Papillomavirus Infections among Young Women in HPV Vaccine Catch-up Cohort}

Supriti Ghosh, Elia Alcañiz Boada, Chris Robertson, Kate Cuschieri, Ramya Bhatia, Seonaidh Cotton

Manipal School of Life Sciences, Manipal, India

Objectives: To determine the human papillomavirus (HPV) genotypes and presence of persistent infections among women who were offered HPV bivalent vaccine through catch-up.

Methods: As a catch-up campaign, young girls up till the age of 18 years were offered the bivalent HPV vaccine in 2008. A total of 100 paired liquid-based cytology samples (LBCs), collected at a median interval of 12 months, were obtained from the Scottish HPV Archive, subjected to DNA extraction (QIAGEN, Germany) and HPV genotyping was performed using the EUROArray HPV kit (EUROIMMUN $^{\mathrm{TM}}$, Lubeck, Germany).

Results: The overall proportion of vaccine cross-reactive HPV types (HPV-16, 18, 31, 33, 45) decreased slightly from 51 to 47\% [95\% CI $-9.7 \%$ to $17.5 \%$ ] in the second smear. Proportion of samples with multiple HPV infections (including non-vaccine HPV types) were observed in over $75 \%$ of the samples. Single infections with the vaccine-HPV types were more persistent than multiple infections (26\% vs $7 \%$ ). Among the vaccine-HPV types, HPV-16 and 31 demonstrated higher persistence compared to acquisition and regression.

Conclusion: High persistence rates of both vaccine and non-vaccine HPV types suggest the need for regular HPV surveillance among immunised women, which would help to determine the efficacy of the HPV vaccine and to explore the natural history of infection among immunised and unimmunised individuals.

\section{Abstract \#75: Comparison of HPV DNA detection in Self- collected vaginal and physician-collected cervical samples in women from South India}

Santhosh Kuriakose

Government Medical College, Kozhikode, India

Objectives: To evaluate the efficacy of self-collected vaginal samples for high-risk HPV in a group of histologically confirmed cervical cancer patients by molecular assays.

Methods: A cross-sectional study was carried out comparing HPV DNA detection by Polymerase Chain Reaction (PCR) in paired selfcollected vaginal samples procured from histologically confirmed cervical cancer cases. The study was carried out among patients 
attending Gynecologic-Oncology Division at Government Medical College, Kozhikode, Kerala, between March 2017 and April 2019. Virology workup was done at Manipal Institute of Virology, Manipal, Karnataka. Self-collected vaginal samples were obtained from consenting patients, followed by cervical sample collection by the clinician. The paired samples were transported at $4-8{ }^{\circ} \mathrm{C}$ to the laboratory. Amplification of LCR/E6/E7 regions of the HPV genome was done by polymerase chain reaction (PCR). The agreement level between paired samples was assessed by the Kappa index.

Results: Amongst the samples collected from 114 cervical cancer cases, HPVDNA was tested positive in cervical samples of 89 (78.1\%) (95\% confidence interval [95\% CI: $69.2 \%-85 \%)$ and selfcollected vaginal samples of $88(77.2 \%)(95 \%$ CI $68.7 \%-83.9 \%)$. The agreement between the two sampling methods was $93.9 \%$ and the kappa value was $0.82(\mathrm{P}<0.001)$ indicating an almost perfect agreement for HPVDNA detection between self-collected and physician-collected samples. The sensitivity of HPV detection using urine samples was 98.9 (95\% CI 93.9\%-99.8\%) and the specificity was $100(95 \%$ CI $86.7 \%-100 \%)$ with cervical sampling as the gold standard.

Conclusion: Self-collection of vaginal samples is an optimum method of detection of HPV DNA in women from third-world countries such as India and ensures equity of cervical cancer screening.

\section{Abstract \#81: Primary Screening for Cervical Cancer by Molecular HPV DNA testing with genotyping-first experience from India}

Vijayalakshmi Ramshankar, Komathy Arun, Soundarya Ravindran, Kanchana Albert

Cancer Institute (WIA), Guindy, India

Objectives: India contributes to $20 \%$ of the global cervical cancer burden and screening is often plagued with lower coverage of the targeted screening population. The utility of partial genotyping on a high throughput automated platform has not been explored in India before. In the current study, the first experience in the implementation of HPV DNA testing with partial genotyping in an ongoing community-based cervical cancer screening program from India is discussed.

Methods: The program aims to offer high-risk HPV DNA testing with genotypes using real-time PCR on a fully automated platform (Cobas 4800, Roche) to 30,000 eligible women (30-65 yrs) for a period of 5 years. Results: We tested community women $(n=3863)$ by qPCR followed by pap and colposcopy.

Results: The prevalence of HR HPV in Indian community women is 6.2\%. Genotype distribution showed 21.2\% HPV 16, 5.4\% HPV 18, $63.9 \%$ other HRHPV and $9.5 \%$-mixed infections. There was a statistically significant association between age groups and HR HPV infection. We found significant mixed infections $(52.2 \%)$ in the age group of 46-50 years age group. About $30 \%$ of the abnormal pap cytology was associated with HPV 16, 3.3\% with HPV 18 and mixed infections and $63.3 \%$ were associated with other HR HPV infections. Genotyping as an objective test can be used for risk stratification of clinically relevant infections requiring additional intervention to help in early detection of cervical cancer in India.

Conclusions: The primary HPV DNA screening using molecular testing is feasible and can help in risk stratification.
Abstract \#240: Predictive value of VIA, HPV genotyping and Colposcopy for CIN in opportunistic cervical screening by primary HPV testing

Harshitha K, Saritha Shamsunder, Sunita Malik, Vijay Zutshi, Anoop Kumar, Rashmi Arora

Department of Obstetrics and Gynaecology, VMMC and Safdarjung Hospital, Delhi

Objective: As the highly sensitive HPV test is adapted in opportunistic cervical screening, it is vital to define the method to best predict high-grade CIN in screen positive women to ensure timely and appropriate treatment.

Methods: We present the results of a cross-sectional observational study of women $\geq 30$ years attending the gynaecology clinic at a referral teaching hospital in New Delhi, India, who had opportunistic cervical screening by primary HPV testing by PCR. Women detected screen positive had HPV genotyping, visual inspection with acetic acid (VIA), colposcopy and biopsy sequentially. The sensitivity, specificity at $95 \% \mathrm{CI}$ and AUC for predicting CIN $2+$ were compared using histopathology as the gold standard.

Results: The HPV prevalence rate in the 1606 women screened was $8.5 \%$. The sensitivity of HPV 16,18 genotyping was $82.1 \%$ (95\% CI $63.11 \%$ to $93.94 \%)$ for VIA $71.43 \%(51.33 \%$ to $86.78 \%)$ and colposcopy was $96.43 \%$ ( $81.65 \%$ to $99.91 \%)$. The specificity was $13.04 \%$ (95\% CI $6.93 \%$ to $21.68 \%$ ) for HPV 16,$18 ; 43.48 \%$ ( $33.17 \%$ to $54.22 \%$ ) for VIA and $30.43 \%$ (21.27\% to $40.90 \%)$ for colposcopy. ROC area under curve for HPV 16,18 was 0.48 (95\% CI 0.38 to 0.57 ), VIA was 0.57 (95\% CI 0.48 to 0.66 ) and colposcopy was 0.63 (95\% 0.54 to 0.72 ). Colposcopy missed one case of CIN 2+, HPV 16,18 missed 5 and VIA missed 8.

Conclusion: Direct colposcopy should be considered in women detected screen positive at the health facility to minimize loss to follow-up and detect and effectively treat high-grade CIN.

\section{Abstract \#163: A New Trend In HPV Diagnosis With Truelab® Point Of Care (POC) micro PCR}

\section{J. Manjula, Chandrasekhar B Nair, Manoj MN, Vinaya R, Amit J}

Bigtec Labs (Subsidiary R \& D of Molbio diagnostics), Rajajinagar, Bangalore, India

Objective: To Provide a Point of Care (POC) microPCR test for detection of HPV (Types 16,18,31 \& 45) infections

Design: Nucleic acid testing is highly sensitive and specific technique for HPV testing but usage is limited due to the need for skilled manpower and elaborate infrastructure. With a point of care platform (POC) system like Truelab®, diagnosis of high-risk HPV is simplified. The Truelab ${ }^{\circledR}$ is a rapid, simple, robust and user-friendly realtime PCR platform offering "sample to result" within an hour. The system is battery operated and has the capability to operate under resource-limited settings. The Truelab ${ }^{\circledR}$ work station comprises of Truelab ${ }^{\circledR}$ real-time quantitative microPCR analyser, Trueprep ${ }^{\circledR}$ Auto Sample prep Device and room temperature stable and disposable Truenat ${ }^{\mathrm{TM}} \mathrm{HPV}, H R$ microPCR tests and Trueprep ${ }^{\circledR}$ Auto sample prep kits. The same platform can be used to develop further tests for other types of HPV if required.

Method: The nucleic acid from the respective swab specimen is isolated with Trueprep ${ }^{\circledR}$ Auto Sample prep device followed by PCR on Truelab ${ }^{\circledR}$ micro-PCR system. At the end of the test run HPV-HR detected or not detected result is displayed.

Results: This test has been validated with Digene HC2 and Sacace kits and has shown a sensitivity of $97.7 \%$ and specificity of $98.9 \%$. 
Conclusions: Overall Truelab ${ }^{\circledR}$ is a handy platform for quick and sensitive detection of high-risk HPV infection.

\section{Abstract \#204: HPV Self Sampling During the Covid Pandemic \\ Vidya K, Sweety, Nalini, Barathi Priya, Sridhar, Latha Balasubramani Department of Oncology, G. Kuppuswamy Naidu Memorial Hospital, Coimbatore, India}

Objectives: To study the feasibility of cervical cancer screening using human papillomavirus (HPV) self-sampling methods during the COVID pandemic.

Design: This was an observational study conducted between March 2020 to January 2021 during the Covid pandemic in women between 30 and 59 years of age in rural Coimbatore.

Methods: During the pandemic, it was impossible to conduct community cancer screening programmes due to social distancing norms. To continue cervical cancer screening, self-sampling technique was adopted. The CARE HPV kit was used for self-sampling. The sampling technique was explained to the participants with the aid of a pictorial representation. The self-obtained samples were then collected by the healthcare workers for analysis.

Results: During this pandemic, a total of 409 women underwent cervical cancer screening using HPV self-sampling methods. Primary HPV screening was done for 368 women. We also used self-sampling as a method to follow 41 women who had been HPV positive 12 months ago. Thirty-one of the 368 women screened primarily were HPV positive (8\%). Eleven of the 41 women who were followed up after 12 months were HPV positive (26\%).

Conclusions: Continued cervical cancer screening in women was possible during the Covid pandemic only by self-sampling. HPV selfsampling provides a safe technique for screening with a reduced risk of exposure to the participant and healthcare personnel. This method was feasible and readily accepted by women.

\section{Abstract \#213: Is An HPV Negative Test by Self Sampling Truly Negative?}

Thasneem P, Nalini C, Sweety Selva Rani, Bharathipriya J, Sridharan V, Vijayalakshmi R, and Latha Balasubramani

G kuppuswamy Naidu Memorial Hospital, Coimbatore, India

Objectives: Self-sampling using the HPV test is being increasingly used as a means of increasing cervical cancer coverage. Various studies have found that women find self-sampling convenient and satisfied with it as a screening option. Self-sampling can either be a sample of cervical cells but more often are of vaginal cells. To determine the compliance of women to the instructions given prior to obtaining a self-sample and to determine the adequacy of the selfsampling technique.

Methods: A cohort of seventy women who attended HPV screening was divided into two equal groups by simple randomization; one group was subjected to self-sampling with cobas ${ }^{\circledR}$ HPV kit, and the second group had cobas ${ }^{\circledR}$ HPV test performed by the healthcare worker. The choice of cobas ${ }^{\circledR}$ was made as this has an inbuilt quality control mechanism which would denote whether no cervical/vaginal cells were made out on the cervical brush that was sent for analysis. Results: Among 70 women screened with cobas ${ }^{\circledR}$ HPV test, 5 (3 out of 35 self-samples and 2 out of 35 healthcare worker collected samples) were HPV positive, and none of the sample was invalid. Out of 5 positive cases, 2 were HPV-16, 1 was HPV-18, and 2 were other hrHPV positive.
Conclusions: This simple study shows that self-sampling provides adequate cellular material for HPV DNA testing. Self-sampling is an efficacious technique that has to be included in cervical cancer screening programmes to increase screening coverage rates. It is an ideal screening mechanism, especially during the Covid pandemic.

\section{Abstract \#214: The Use of HPV Self-Sampling to Increase Follow- Up Rate: A Feasibility Study}

Thasneem P, Nalini C, Sweety Selva Rani, Bharathipriya J, Sridharan $\mathrm{V}$, and Latha Balasubramani

G Kuppuswamy Naidu memorial hospital, Coimbatore, India

Objectives: This study investigated the potential improvement in cervical cancer screening follow-up by outreach program and HPV self-sampling. Participant's perceived belief towards cervical cancer screening was also studied using a validated questionnaire.

Design: A prospective, observational study was conducted on women screened HPV positive from October 2017 to December 2019, in the rural and urban areas of Coimbatore, India.

Methods: Women who were HPV positive on initial screening were invited to attend the health centre for their 12-month follow-up. Those who did not respond were traced and offered self-sampling with Care HPV at their residence to study improvements in the follow-up.

Results: Among the 2977 women who were screened with HPV test, $210(7.05 \%)$ were HPV positive. Only 23\% underwent colposcopy, while others were lost to follow-up and did not respond to repeated calls. When self-sampling was offered as a follow-up option, $60 \%$ complied with $27.8 \%$ showing a repeat positive test. Conducting colposcopy in the community showed improvement in follow-up from $23 \%$ to $45.2 \%$. The time interval between two positive HPV tests was an average of 14.9 months.

Conclusions: This is one of the few studies reporting follow-up of HPV-positive women by outreach program and by providing selfsampling as a mean to reduce the default rate. Offering self-sampling found to improve the follow-up rate, which is equally efficacious as the clinician sample.

\section{Abstract \#298: The clinical value of HPV genotyping in triage of women with high-risk HPV-positive self-samples for cervical cancer screening in China}

LI Jingran, Ruifang Wu, Ling LI, Zhixin Ling, Zhijun ZHANG, Rong LIU, Yilan SHA, Jihong DENG, Ruifang AN, Songling ZHANG, Bei LIN, XiaofengZHAO, HuiDU, Chao ZHAO, Mingzhu LI, Yun ZHAO, Lihu WEI.

Peking University People's Hospital, Xicheng District, Beijing, P.R. China

Objective: To investigate the clinical value of high-risk HPV-positive triage in the mode of vaginal self-sampling for cervical cancer screening.

Methods: 19,485 women aged $30 \sim 59$ years with vaginal selfsampling HPV testing as primary cervical cancer screening on internet-based was performed from September 2018 to December 2019 in 13 provinces, municipalities, and autonomous regions of China, which organized by Peking University People's Hospital and Peking University Shenzhen Hospital. The women who participated in the screening registered, read, and signed the informed consent on the cervical cancer screening website (http://47.106.227.241/). A unique $\mathrm{QR}$ code was obtained for taking the sampler. The specimens were taken by women themselves from the vagina, where at the screening site or mail sampler to them. 14 types of high-risk HPV 
(HPV16, 18, 31, 33, 35, 39, 45, 51, 52, 56, 58, 59, 66, 68) were tested and positive patients were referred to colposcopy with multi-spot biopsy, and the detection of high-risk HPV for cervical high-grade lesions (HSIL) were analysed. A questionnaire was conducted to investigate the self-sampling acceptance and sensation after sampling. Results: A total of 19,485 women were enrolled, $29.83 \%$ of them were never screened, $15.24 \%$ with a primary school education or below. A total of 19,485 vaginal self-samples were collected, 23 cases $(0.12 \%)$ unqualified samples, and 19,452 (99.83\%) qualified samples, 10 cases were barcode error. In questionnaire data, $95.27 \%$ of women felt self-collection was easy to operate; $82.32 \%$ had no discomfort when using a self-sampling brush; $64.11 \%$ women were more likely to choose self-sampling for cervical cancer screening in the future. Overall high-risk HPV prevalence was $13.70 \%$ in this group with 13 provinces of China. The highest prevalence of high-risk HPV positive was $21.75 \%$ in Inner Mongolia Autonomous Region, and the lowest rate was $8.89 \%$ in Guangdong Province. Among 1,983 women undergone colposcopy-directed biopsy, 157 cases $(7.92 \%, 157 / 1983)$ of HSIL + lesions including 5 cases $(0.25 \%, 5 / 1983)$ with cervical cancer, and 521 cases $(26.27 \%, 521 / 1983)$ with LSIL were detected. The sensitivity of self-sampling HPV testing for detecting HSIL + was $99.36 \%$, with a $90.21 \%$ of specificity. Detecting value of different HPV genotyping combinations in triage of HPV-positive women for HSIL + is shown in Table 1.

Table 1 Strategies for triage of HPV-positive women for detecting HSIL+

\begin{tabular}{lllllll}
\hline & $\begin{array}{l}\text { Sensi- } \\
\text { tivity } \\
(\%)\end{array}$ & $\begin{array}{l}\text { Speci- } \\
\text { ficity } \\
(\%)\end{array}$ & $\begin{array}{l}\text { Missed } \\
\text { diagnosis } \\
(\%)\end{array}$ & $\begin{array}{l}\text { Misdiag- } \\
\text { nosis } \\
(\%)\end{array}$ & $\begin{array}{l}\text { PPV } \\
(\%)^{\#}\end{array}$ & $\begin{array}{l}\text { NPV } \\
(\%)^{\#}\end{array}$ \\
\hline HPV16/18 & 56.69 & 82.91 & 43.31 & 17.09 & 22.19 & 95.70 \\
HPV16/18/33 & 67.52 & 78.92 & 32.48 & 21.08 & 21.59 & 96.58 \\
HPV16/18/33/58 & 78.34 & 66.10 & 21.66 & 33.90 & 16.58 & 97.26 \\
$\begin{array}{l}\text { HPV16/18/33/58/ } \\
\text { 31 }\end{array}$ & 83.44 & 61.83 & 16.56 & 38.17 & 15.82 & 97.75 \\
\hline
\end{tabular}

\# PPV: positive predictive value; NPV: negative predictive value

Conclusion: The mode of cervical cancer screening with vaginal selfsampling HPV detection is feasible, with high screening efficiency and acceptability, and combining HPV16/18 with HPV33 and HPV33 might be used as a triage method in China.

\section{Abstract \#302: Analysis of the agreement between self- and clinician-collected samples for the human papillomavirus detection}

QihuiFeng, Yue Wang, Keyong Bao, Peng Zuo, Mingzhu Li, Chao Zhao, Jingran Li, Na Wang, Yi Zhu, Lihui Wei

Peking University People's Hospital, Xicheng District, Beijing, P.R. China

Objective: The aim of the study was to compare the agreement between the self-sampling and clinician-sampling for HPV test and the possible factors influencing the agreement.

Methods: A total of 1083 women aged 25-65 were recruited. All the participants were asked to complete a case report form first. Then they were taught to collect specimens for HPV test by themselves, followed by the clinician-collected samples for HPV test (using four different materials) and cytological analysis. Participants whose HPV and TCT results meet the indications were referred to colposcopy examination.
Results: Among the 1083 women aged 25-65 years the prevalence of HPV using Roche Cobas 4800 HPV DNA test or fluorescence quantitative PCR was $15.2 \%(165 / 1083)$ and $17.1 \%(185 / 1083)$. The percent positive for self-collected samples using fluorescence quantitative PCR was $18.2 \%$. The overall agreement of the self- and clinician sampling was $94.7 \%$. The agreement of the different subtype of HPV between self- and clinician-collected samples was moderate to excellent (Kappa: 0.664-0.969), among which the 18 subtype was the highest. The sensitivity to find high-grade lesion with self- and clinician-collected samples are both $100 \%$.

Conclusion: The agreement in detecting HPV between the self- and clinician sampling is good. It is a feasible way to make the cervical cancer screening and more convenient and widespread.

Abstract \#303: Study on the value of Human papillomavirus test and 16/18 typing detection in the cervical cancer screening

Yun Zhao, Mingzhu Li, Jingran Li, Chao Zhao, Yu Wang, Ruijun Tang, Lihua Ren, Yanyan Li, Chunguang Li, Xiuhua Zhao, Jianliu Wang, Lihui Wei

Peking University People's Hospital, Xicheng District, Beijing, P.R. China

Objective: To evaluate the value of HPV testing and HPV16/18 genotyping in the primary screening of cervical cancer.

Methods: HPV testing and Pap test were performed on 11,846 women in Sichuan Province, Zhejiang Province, Beijing and Inner Mongolia, China, among them 528 women underwent colposcopy. The sensitivity and specificity of HPV test were analysed according to the pathological result $\geq \mathrm{CIN} 2$ as the gold standard for the cancer screening.

Results: Among the 11,846 women who were screened, the positive rate of HPV test was $14.20 \%(1685 / 11846)$, and the positive rate of HPV 16 and/or 18 infections was 2.47\% (293/11846). There were 79 women with $\geq$ CIN2, 0.67\%(79/11846) incidence rate. Among them, HPV-positive infection rate was $93.67 \%$ (74/79), and HPV16 and/or HPV18-type positive rate was $62.03 \%$ (49/79). The sensitivity of HPV-positive screening was $93.67 \%$, and the sensitivity of HPV16/18 screening was $90.74 \%$, which was higher than that of TCT screening by $81.01 \%$. The highest incidence rate of $\geq$ CIN2 was $0.12 \%(14 /$ 11846), among the age 50-54 years, followed by the 35-39 age group, which was $0.11 \%(13 / 11846)$. The incidence rate of $\geq$ CIN2 stage of Yi nationality in Sichuan hospital was $1.14 \%$ (8/700), higher than that of Han nationality $0.74(16 / 2161)$, and the incidence rate of CIN2 stage of Manchu nationality in Mentougou was $3.57 \%(2 / 56)$ higher than that of the Han nationality $0.71 \%(21 / 2938)$. There was no significant difference between the Mongolian incidence rate $0.80 \%(2 /$ $251)$ and that of the Han nationality $0.617 \%(1536 / 2454)$ in Inner Mongolia.

Conclusion: HPV detection and 16/18 typing can be applied for the cervical cancer initial screening and improve the screening sensitivity.

\section{HPV Vaccination}

Abstract \#108: A study done in tertiary care center to survey barrier to HPV vaccine acceptance

Pratibha Kumari, Sangeeta Pankaj, Satya Kumari, Kavya Abhilashi, Jyotsna Rani

Indira Gandhi Institute of Medical Sciences, Patna, Bihar, India 
Objectives: Cervical cancer is the second largest cause of cancer deaths in women worldwide. It takes decades to develop from persistent infection with high-risk types of human papillomavirus (HPV). Thus, prophylactic vaccination against HPV is an attractive strategy required to prevent development of cervical cancer. This study was conducted to study barriers in acceptance of HPV vaccination among medical staffs by running a HPV vaccination Program.

Methods: This study was conducted in a tertiary care hospital among medical staffs children. The HPV vaccination programme was started in May 2018 till January 2020. Bivalent Cervarix vaccine was used and targeted girls were from 9 years till 26 years of age. A total of 61 girls were vaccinated through this programmme.

Result: HPV Vaccine uptake was found to be high in routine population girls around $93 \%$ receiving all doses of vaccine than girls of catch up group.

Conclusion: Lack of information, education, fear of adverse reaction and high cost related to HPV vaccination among medical staffs can jeopardise HPV vaccine uptake among their children. If medical staffs children are vaccinated they are more likely going to recommend vaccination to their patients, relatives and their nearby ones. Thus, it is essential to take time and actively listen and answer their questions and concern related to vaccination. We forget that they are also part of public and making them aware about HPV vaccination will directly help to clear all doubts of general population. So prophylactic vaccination against HPV is an attractive strategy required to prevent development of cervical cancer in Indian women.

\section{Abstract \#109: Safety and acceptance of HPV vaccine: A hospital- based survey at tertiary care centre}

\author{
Manju LataVerma, Uma Singh, Prachi Rai, Rekha Sachan, Pushp \\ Lata Sankhwar
}

Department of Obstetrics and Gynaecology, King George Medical University, Lucknow, India

Objectives: To evaluate the safety and acceptance of HPV vaccines in northern India

Methods: A pilot intervention study was done from June 2016 to June 2017 in the department of Obstetrics and Gynecology of King George Medical University, Lucknow, Uttar Pradesh, India. First acceptability was determined by filling questionnaire by taking face-to-face interview and subjects who accepted for the vaccines, were provided vaccines (Cervarix). Various side effects in terms of pain at injection site, erythema, induration, fever, myalgia, etc. were assessed after every injection. Ethical approval was obtained from the Institutional Ethical Committee of the KGMU, Lucknow, Uttar Pradesh, India. (1689/Ethics/R cell/17). For continuous variables, mean with standard deviation was used. For categorical variables frequencies and percentages were used. IBM SPSS statistics applied.

Results: A total of 302 cases were face-to-face interviewed, amongst which, 70 cases $(23 \%)$ showed acceptability. Total cervarix doses given were 196. The most common side effect was pain at injection site followed by induration $3.5 \%$ and $1.5 \%$, respectively.

Conclusions: HPV vaccines are quite safe and safety is not a major concern for nonacceptability of HPV vaccines.

\section{Abstract \#211: Overcoming the Barriers of HPV Vaccination During Covid-19 Pandemic at Delhi State Cancer Institute In India}

Vinita Kumar Jaggi, Pradeep Kumar Tiwari, Manoj Kumar

Delhi State Cancer Institute, Delhi, India
Objectives: India alone bears $23 \%$ of the global cervical cancer burden. Cervical cancer is the first cancer to be shown to be $100 \%$ attributable to a virus; oncogenic human papillomaviruses (HPV), particularly types 16 and 18 . In India, the most common oncogenic types are HPV 16 and 18 accounting for more than $90 \%$ cases. In India, population-based cervical cancer screening is largely non-existent in most regions due to competing healthcare priorities, insufficient financial resources and a limited number of trained providers. Hence, most of the cases present in advanced stages of the disease, thus leading to increased mortality and reduced survival.

Methods: Introduction of HPV vaccination (Cervarix) in Delhi State Cancer Institute offers great promise to reduce the burden of cervical cancer in conjunction with regular screening methods. Two doses of HPV vaccine (Cervarix) given free of cost at DSCI Delhi to adolescent girls aged 9 to 13 years. Even during the COVID-19 pandemic situation, the HPV vaccination program does not hampered, adolescent girls were highly motivated and vaccinated in large numbers at DSCI.

Results: During COVID-19 pandemic, over 4000 adolescent girls were vaccinated at DSCI, Delhi, in which around 2800 girls received first dose and 1200 received their second dose of vaccine. This becomes possible through regular community and school-based awareness campaigns run by DSCI.

Conclusion: Prevention of cervical cancers with two-dose HPV vaccination and early detection of precancerous cervical lesions of the eligible population through screening and their appropriate treatment with a single-visit 'screen-and-treat' approach appear to be promising for low-middle-income countries including India.

\section{Abstract \#227: Has The Human Papillomavirus (HPV) Immunization Programme Improved Obstetric Outcomes In Spontaneous Delivery? An Ecological Study.}

Xiaoqian $\mathrm{Xu}^{1,2}$, Andrea Woolner ${ }^{1}$, Sohinee Bhattacharya ${ }^{1}$, Seonaidh Cotton $^{3}$, Fanghui Zhao ${ }^{* 2}$, Margaret Cruickshank ${ }^{* 1}$

${ }^{1}$ Aberdeen Centre for Women's Health Research, University of Aberdeen, Aberdeen, United Kingdom, ${ }^{2}$ Department of Cancer Epidemiology, National Cancer Center/National Clinical Research Center for Cancer/Cancer Hospital, Chinese Academy of Medical Sciences and Peking Union Medical College, Beijing, China, ${ }^{3}$ Health Services Research Unit, University of Aberdeen, Aberdeen, United Kingdom

Objectives: To measure the rates of adverse obstetric outcomes in spontaneous delivery in a population of young women with high uptake of the bivalent human papillomavirus (HPV) vaccine.

Study Design: This was a population-based ecological study with data from the Aberdeen Maternity and Neonatal Databank, UK. All women born between 1986-1996 with spontaneous singleton live birth at age 20 to 30 years were included for analysis. Exposure was defined according to maternal year of birth and HPV immunisation eligibility: pre-immunisation cohort (1986-1990), catch-up immunisation cohort (1991-1994) and routine immunisation cohort (1995-1996). Outcome were defined as spontaneous preterm birth (PTB), low birth weight (LBW) and pre-labour preterm rupture of membranes (PPROM). Generalized estimating equation models were applied, adjusted for deprivation, smoking status, marital status, body mass index, parity, maternal age and year of infant delivery.

Results: A total of 6515 spontaneous singleton live birth were included in final analysis. Compared with the pre-immunisation cohort, the catch-up cohort showed an adjusted odds ratio (a OR) of 0.64 (95\% confidence interval, 0.40-1.03) on spontaneous PTB. The aOR was $0.71(0.28-1.77)$ in the routine cohort. The corresponding $\mathrm{aOR}$ in the catch-up and routine cohorts were $0.88(0.54-1.45)$ and 
$0.51(0.16-1.62)$ for spontaneous LBW and $1.62(0.58-4.54)$ and 1.51 (0.21-11.01) for spontaneous PPROM.

Conclusions: The potential reduction in PTB among spontaneous singleton live birth may be associated with a high coverage of bivalent HPV immunization programme. However, this additional benefit to cancer prevention needs further demonstration in the emerging fully immunised cohorts.

\section{Abstract \#292: Challenges of Audio-Visual Consenting Process: Experience From HPV Vaccine Trial in India}

Parul Jaiswal, Swati Tomar, Jayashree Natarajan, Shachi Vashist, Rajesh Kumari, Anju Singh, Neerja Bhatla

Department of Obstetrics and Gynecology, All India Institute of Medical Sciences, New Delhi, India

Objective: To describe the procedure and difficulties of audio-visual consenting process during a phase II/III HPV vaccine trial among healthy children between 9-26 years.

Design: Retrospective cross-sectional single-centre study

Method: Audio-visual consenting procedure was conducted for male and female subjects aged 9-26 years, or their parents (wherever applicable), recruited in a phase II/III quadrivalent HPV vaccine trial in New Delhi from March 2019 to January 2021. Initially, some critical component or other was omitted. A checklist was prepared of the salient features required to be captured. The camera was set up in a designated sound-proof room. Data were extracted from the archived trial database.

Results: Out of 350 subjects enrolled, 80 (22.87\%) declined to participate due to refusal from parents and the COVID-19 pandemic, while $51(14.57 \%)$ were excluded from the trial on account of concomitant medical illness. Of the 219 consents analyzed (participation rate $=73.24 \%), 139(63.47 \%)$ were females, and $80(36.53 \%)$ were males; 75 subjects $(34.25 \%)$ were accompanied by their mothers, 42 $(19.18 \%)$ by their fathers, while rest were $>18$ years. Most preferred language was Hindi $(142 / 219,64.84 \%)$. Mean \pm SD duration of the interview was $26.58 \pm 9.19 \mathrm{~min}$. Medical social workers were the major motivators $(111 / 219,50.68 \%)$ who facilitated the consent procedure by providing background information to subjects. Twentyfour pairs $(13.71 \%)$ of siblings could be interviewed together.

Conclusion: Audio-visual consenting is a necessary skill for conducting clinical trials. Messages have to be conveyed crisply and succinctly in easily understandable language, while dealing with individual concerns of subjects.

\section{Abstract \#299: Effort to promote the use of HPV vaccine in China Lihui WEI \\ Peking University People's Hospital, Xicheng District, Beijing, P.R. China}

Objective: Cervical cancer is a major threat to women's health in China. Eliminating cervical cancer in China is a huge challenge, among them the application of HPV vaccine is an important part.

Methods: There are currently four HPV vaccines in China, E-coli bvHPV vaccine (made in China, qv-HPV (GSK) and 9v-HPV vaccine (MSD). To observe the immunogenicity, efficacy and safety of four vaccines after vaccination in China

Results: At 7 months after vaccination, all vaccinated subjects had the same immunogenic response to either HPV16 or HPV18, ranging from 96 to $100 \%$, and antibody production in girls aged 9-14 years was 2-3 times higher than that in adult women. The efficacy of four vaccines of against CIN2 + was $87.3 \% \sim 100 \%$, the prevention of
HPV-associated infection reached $96 \% \sim 97 \%$ at 12 months, also safe in women aged $18 \sim 45$ years in two kinds of bv-HPV and qvHPV vaccine of clinical trail in China. Clinical trials of 9v-HPV vaccine are underway. Now HPV vaccination is voluntary and selfpaid in China. In order to promote the application of HPV vaccine in China, we have developed a "Chinese expert consensus on clinical application of human papillomavirus vaccine".

Conclusions: In clinical study HPV vaccine showed excellent efficacy, safety and immunogenicity in Chinese women. We will continue to strengthening screening, and pushing forward with HPV vaccination

\section{Abstract \#311: Feasibility and acceptability of quadrivalent HPV vaccine for adolescent girls in rural West Bengal}

Sreeya Bose ${ }^{1}$, Manisha Verneker ${ }^{1}$, Dipanwita Banerjee ${ }^{1}$, Krishnendu Gupta $^{2}$, Ranajit Mandal ${ }^{1}$

${ }^{1}$ Department of Gynaecological Oncology, Chittaranjan National Cancer Institute, Kolkata, ${ }^{2}$ Department Of Obstetrics and Gynaecology, Vivekananda Institute of Medical Sciences, Kolkata, India

Objective: To evaluate the feasibility, acceptability and safety of two doses' of HPV vaccination in adolescent girls. Another objective was to evaluate the awareness about the cervical cancer and its vaccine in the community.

Design: Prospective cross-sectional study

Method: The eligible girls, between 9-14 years of age were recruited after their parents signed a consent form and the participants had signed an assent form. Vaccine doses were recommended at 0 and 6 months. To estimate the feasibility, successful completion of two doses by at least $80 \%$ of the girls will be measured. To measure the acceptability, either of the parents will be called along with their daughter at 7 th month and will be asked to fill up a questionnaire.

Results: The project was started in July 2017 and is ongoing. Total 1667 children were vaccinated till February 2021. The respondents were asked to report any side effects in the next $24 \mathrm{~h}$. All the children and their parents were contacted on Day 7 and Day 30 post-vaccination period and were asked about any side effects and their level of satisfaction. Second dose was completed for 1567 children by March 2021. After their second dose, participants with their parents will visit Chittaranjan National Cancer Institute, Kolkata, to fill a questionnaire, hence recording their level of satisfaction.

Conclusion: By establishing these evidences from such pilot projects, HPV vaccination programmes could be made more sustainable within the context of a comprehensive approach to cervical cancer prevention and control as developed by WHO (World Health Organisation).

\section{Molecular Markers}

Abstract \#110: Role of Human Papillomavirus oncoproteins E6 \& E7 in screening and diagnosis of Cervical carcinoma

Sangeeta Pankaj, Vijayanand Choudhary, Jyotsna Rani, Pratibha Kumari, Kavya Abhilashi, Satya Kumari

Indira Gandhi Institute of Medical Sciences, Patna, India

Objectives: Persistent high-risk human papillomavirus is an established cause of cervical cancer in $99.7 \%$ cases. In most of the cases, integration of HR-HPV genome gives rise to fusion transcripts comprising of viral oncogenes E6/E7 and adjacent cellular sequences. To study genotypes of HPV in cervical pre-cancer and cancer amongst women of Bihar \& observe expression of oncogene E6 \& E7 
for evaluation of the effectiveness of molecular method in screening and diagnosis of cervical cancer.

Methods: This prospective observational study enrolled 100 subjects including 50 patients with cervical neoplasia confirmed by histopathology and 50 as controls. All patients underwent co-testing and levels of E6/E7 oncoproteins were determined. The diagnostic value of HPV E6/E7 protein assay was compared with the clinical diagnosis from cytology \& the gold standard of cervical biopsy histopathology.

Results: A total of 46 of 50 cervical cancer patients reported HR HPV positive and 17 of them were positive for E6/ E7 oncoproteins. Amongst controls, 44/50 with normal cervical morphology followed with HPV report and 30 of them showed HR HPV positivity. E6 was found in 23/30 subjects while E7 was positive only in 2. On histology, 1/44 patient was diagnosed with CIN3 and none of them had invasive carcinoma. The sensitivity of the HPV E6/E7 assay was observed lower than HR-HPV testing but with a higher specificity. HPV testing \& E6/E7 assay had higher sensitivity than cytology reporting.

Conclusion: HPV E6/E7 oncoprotein levels is a novel biomarker for cancer cervix with higher specificity.

\section{Abstract \#124: Role of p16-An Aid in Management of CIN2 in Women of Childbearing Age}

Anannya Kar, Padma Eedarapalli, Victoria Perera, Rachana Dwivedi

St. Mary's Hospital, London, United Kingdom

Objectives: There is increasing evidence that cervical excisions $>10 \mathrm{~mm}$ in depth are associated with increased risk of secondtrimester miscarriage/pre-term birth, necessitating an approach to determine management options to balance against risk of progression of pre-malignant disease. Aim of study was to determine outcome of management options of CIN2 in women of childbearing age using P16 as a triage tool. Outcome measures were follow-up results at 6 months including HPV test-of-cure, recurrence or persistence of high-grade lesion and additional treatment.

Methods: Retrospective study of women of childbearing age with CIN2 diagnosed on histology over 5-year period between 2014-2019 was conducted. Primary standard was all histology showing CIN2 is stained for p16. Criteria for conservative management were based on recommendations by NIHR regional guidelines for management of CIN2 in women of childbearing age.

Results: Of 300 patients, a preliminary analysis showed demographics of $70 \%$ to be $<35$ years and $40 \%$ smokers. $95 \%$ had histological staining for p16. Amongst patients with p16-positive staining, 54\% underwent cold coagulation, 34\% underwent large-loop excision of transformation zone (LLETZ). 52\% had excision depth $>10 \mathrm{~mm} ; 10 \%$ had $>15 \mathrm{~mm}$. Colposcopic opinion-histology correlation matched for $51 \%, 98 \%$ had $>=2$ biopsies taken indicative of increased diagnostic accuracy. All p16-negative cases were discussed in MDT for consideration of conservative management.

Conclusions: p16 staining is useful in guiding management of women with CIN2. In this sample, majority of histology stained positive for p16 who were managed with either cold coagulation or LLETZ. With increasing routine application of p16 staining, patients with CIN2 and negative p16 staining may be considered for conservative management via MDT if certain criteria are met to reduce obstetric morbidity, enable patient counselling and informed decisionmaking.
Abstract \#176: P16/K167 Dual Staining In Low-Grade Cervical Smears For Detection Of High-Grade Lesions.

Saloni Chadha, Gauri Gandhi

Department of Obstetrics \& Gynaecology, Maulana Azad Medical College and Lok Nayak Hospital, New Delhi, India

Objectives: To triage low-grade cervical smears (ASCUS/LSIL) by dual staining and to find the sensitivity and specificity of this method for detection of high-grade cervical intraepithelial neoplasia.

Design: Prospective cross-sectional study

Methods: In this study, we evaluated a total of 89 women with lowgrade smears (54 ASCUS,35 LSIL) recruited from Lok Nayak Hospital and ICMR-NICPR from November 2017 to March 2019. All patients underwent Colposcopy and guided cervical biopsy. Histopathology was used as gold standard. All were subjected to P16/ Ki67 Dual staining (excluding 4 with inadequate sample) using Roche ${ }^{\circledR}$ kit. We then used this method to detect high-grade cervical lesions. Data was entered in excel and analysed using SSPS version 22. The sensitivity, specificity, positive predictive value, negative predictive value, and accuracy of the triage test were calculated with histopathology as a gold standard.

Results: Overall in all low-grade smears sensitivity, specificity, and accuracy of dual staining, was found as $66.7 \%, 84.8 \%$ and $83.5 \%$, respectively; $(\mathrm{p}=0.01)$. The positive predictive value and negative predictive value for the test was estimated to be $25 \%$ and $97.1 \%$, respectively.

Conclusions: Overall in all low-grade smears, the specificity and accuracy of the test was higher than sensitivity which was low. It was thus concluded that it is an effective triage method.

\section{New Technologies in HPV Testing}

Abstract \#139: Comparison between conventional cytology and a new molecular genetic test in a high Andean population at 3400 m.a.s.l.

Enrique Alvirez Freites, Michael Dean, Fernando Miranda, Heidi Santos, Luis Felipe Condori, Lisa Garland

\section{UNIVERSIDAD ANDINA DEL CUSCO, Peru}

Objectives: Ccasacunca, Cusco, Peru a peasant community at 3400 masl, with 640 inhabitants. They practice sexual and reproductive activity among themselves, which makes them a population of interest to study HPV. In order to compare cytological findings with a new molecular genetic screening test a cross-sectional descriptive study was established.

Methods: Pap smears and DNA samples from uterine cervix were collected prior informed consent. A high-throughput next-generation sequencing whole-genome method was applied. DNA was used for overlapping amplicon sequencing of the genome. Subsequently, a descriptive analysis was performed.

Results: The average age is $50.96 \pm 16.92 .13$ settlers ever had Papanicolaou, being the first evaluation at $37.54 \pm 13.57 \mathrm{y} / \mathrm{o}$. The average number of Pap smears is $1.46 \pm 1.13 .95 .8 \%$ never used oral contraceptives. First sexual intercourse at $18.09 \pm 2.23$. the average number of sexual partners being $1.09 \pm 0.43$. By cytology $58.33 \%$ are negative for intraepithelial injury, $8.33 \%$ with atypical glandular cells, $20.83 \%$ moderate inflammation, $4.17 \%$ severe inflammation/vaginosis and $4.17 \%$ with atypical squamous cells. $46 \%$ of patients are positive for Hybribio test, having 33,33\% low risk $(6,53$, $66,91,61,55,67,90)$ and $12.5 \%$ high risk $(16,68 \mathrm{~B}, 31) \mathrm{HPV}$.

Conclusion: Sociodemographic factors, sexual and reproductive behavior seem to play a role in the prevalence of HPV, this being the first experience in this community. Molecular genetic testing was 
much more specific and sensitive to detect patients at risk for cervical cancer lesions. Additional studies are necessary to compare viral banks in other high Andean populations.

\section{Cervical Cancer Screening}

\section{Innovations in Cervical Cancer Screening}

Paper number 309: Attitude of Female Doctors Towards Pap Smear for Cervical Cancer Screening in a Tertiary Hospital: A Nigerian Study

Dr. Eli Sukarime, Ekiyor C2, Kalio DGB3, Briggs NCT3, Ela G3.

Mother, Baby And Adolescent Care Global Foundation.

CEO RAHI Hospital. ${ }^{2}$

Department of Obstetrics And Gynaecology, Rivers State UniversityTeaching Hospital. ${ }^{3}$

Department of Community Medicine, Rivers State UniversityTeaching Hospital. ${ }^{4}$

Correspondence: Dr Kalio DGB (MB BS, FWACS, FICS)

Email: kaliodango@yahoo.com

\section{Abstract:}

Background: Doctors at all levels of care are directly or indirectly involved in the counselling and screening of patients with regards to prevention of cervical cancer. Despite the fact that Pap smear screening modalities have reduced the incidence of mortalities from cervical cancer some female medical doctors are indifferent when it comes to accepting this screening method.

Aim: To ascertain the attitude of female doctors towards Pap smear screening modalities at the Rivers State University Teaching Hospital (RSUTH).

Method: It was a cross-sectional study amongst female doctors randomly selected at the Rivers State University Teaching Hospital over a 1-year period. Informed consent was obtained from the subjects. The information obtained was coded and analysed using SPSS version 25.

Results: Three hundred questionnaires were distributed and 280retrieved. The mean age from the study was 31.1 years. The maximum number of children was 5 . Two hundred and sixty-nine $(96.1 \%)$ of the female doctors accepted pap smear, of which 94 (33.5\%) had had Pap smear in the past, while $185(66.1 \%)$ had not had Pap smear screening for cervical cancer. One hundred and forty $(50 \%)$ of the female doctors preferred an alternative to pap smear.

Conclusion: This study revealed that the acceptance of pap smear for the screening of cervical cancer by female doctors at the RSUTH was $96.1 \%$., though those that had pap smear screening for cervical cancer in the past were below average. Half $(50 \%)$ of the female doctors preferred alternative to Pap smear. Effort should be made by the government and stake holders to make other screening methods for the prevention of cervical cancer especially amongst female doctors available.

Keywords: Attitude, Female doctors, Pap smear, Cervical cancer, RSUTH.

\section{Abstract \#4: Triage of HPV-positive screen results for optimization of colposcopy}

Jeff Andrews, Alice McGee, Theresa Freeman-Wang, Deirdre Lyons, Julie Bowring, Jennifer Byrom

Women's Health \& Cancer, BD Life Sciences and Global Health, United Kingdom
Objectives: An analysis of published science about the clinical value of triage options after HPV-positive screen results.

Methods: MedLine was searched from 2001 through 2020 for relevant studies, supplemented by hand-searching of retrieved article reference lists. Eligible studies included prospective studies of women and retrospective studies of residual specimens from women that were tested using HPV genotyping tests. Outcomes were CIN2/CIN3 or CIN2 + /CIN3 + or invasive cervical cancer. The comparator was cytology as a triage for HPV-positive results.

Results: Reporting genotyping provides discrimination of both current and future CIN3 + risks. Genotyping combined with cytology improves risk discrimination for Bethesda categories NILM, ASCUS, LSIL. Improvements in referral rates may be achieved. Immunohistochemical staining with p16 and Ki67 has good evidence for triage of HPV-positive screening results. Methylation of viral HPV and host markers has fair evidence for triage of HPV-positive screening results and may be applicable to self-collected samples. Surrogate estimates of viral load may provide additional risk discrimination.

Conclusion: Based on quality-evaluated studies that met inclusion criteria, genotyping combined with cytology discriminates risk and supports risk-based clinical action steps by the principle of equal management for equal risk. Viral load could be used to augment genotyping risk discrimination. P16/Ki67 staining could be used as an adjunct to cytology or to replace cytology. Methylation tests could be utilized as an adjunct triage test with cytology, or to replace cytology as triage. Models for different management paradigms are described.

\section{Abstract \#12: The Performance of Real-time Optoelectronic Device (TruScreen (8) for Early Diagnosis of Cervical Cancer and Precancerous Lesions: A Multi-center Evaluation}

Fei Chen; Yuqian Zhao; Wen Chen; Tingyuan Li; Jun Liu; Jiandong Wang; Hongyan Guo; Yu Zhang; Jinghe Lang

\section{Truscreen Pvt. Ltd, Australia}

Objective: The TruScreen is a real-time device differentiating cervical tissue by electrical and optical signals, which might be an option for regions that are difficult to build laboratory-based screening system for cervical cancer. To assess the clinical performance of TruScreen in detecting high-grade cervical lesions in Chinese women. Methods: During May 2017 and August 2018, women visited the gynaecology clinics at 5 top hospitals for cervical cancer screening or for colposcopy were invited to participate. The voluntary women all underwent TruScreen examination, liquid-based cytology and hrHPV DNA testing, and colposcopy. Biopsy was performed when cytology was LSIL or worse, or the findings of TruScreen or colposcopy were positive. Histopathology diagnosis was the golden standard.

Results: 1,022 women were included for data analysis. $62.4 \%$ of the women were cytological ASC-US +, 91.3\% were high-risk HPV positive. TruScreen test was abnormal in $41.5 \%$ women. Among the 1022 women, 152 women were diagnosed with CIN2 and 99 were diagnosed with CIN 3 + lesions. The CIN2 + and CIN 3 + yields were much lower for positive HPV with negative TruScreen, compared with NILM cytology (CIN2 + : $4.4 \%$ vs.13.4\%; CIN3 + : $1.0 \%$ vs. $4.0 \%, \mathrm{P}<0.05)$. The sensitivity and specificity for HPV testing and TruScreen co-testing for CIN2 + and CIN3 + was $99.6 \%$ and $9.4 \%, 100.0 \%$ and $7.9 \%$, respectively. The strategies of triaging HPV-positive women with TruScreen in detecting CIN2 + or CIN3 + showed high sensitivities as $89.3 \%$ or $91.9 \%$.

Conclusion: TruScreen test as a simple and rapid real-time method for early diagnosis of cervical cancer and precancerous lesions is very promising. The combination of hrHPV DNA testing and TruScreen, or the strategies of triaging HPV-positive women with TruScreen, 
also demonstrated a better accuracy than that of hrHPV and cytology co-testing.

\author{
Abstract \#33: Combined Pap smear and Visual Inspection \\ with Acetic Acid-Based Screening for Cervical Cancer \\ in a Tertiary Care Hospital \\ Payal Keswarpu \\ Dalrada Health, Bangalore, India
}

Objectives: To evaluate if combined screening with Pap smear and VIA had any added benefit in a tertiary care setting.

Methods: This is a cross-sectional study involving a total of 100 cases who would be recruited in the outpatient department when presenting with various gynecological complaints and fulfilling the study inclusion criteria. An experienced gynecologist conducted the procedure. Brief clinical history followed by visual inspection with acetic acid using a disposable kit and Pap smear test were done on all cases for comparison of results. Biopsy was done if VIA was positive or Pap smear showed any abnormality.

Results: VIA was negative most of the time, approximately $96 \%$ of cases. And this result was concordant with Pap test showing inflammatory smears with no intraepithelial lesions and biopsy being negative. About $30 \%$ of cases had discharge clinically consistent with candidial infection; however, only few pap smears showed budding yeast.

Conclusion: Involving a specialist might reduce the false positivity of VIA. Not all erosive unhealthy-looking cervix which bleeds on touch was found VIA/PAP positive. PAP might not pick up all candidial infections. Combining both tests will reduce the number of visits to the hospital as a VIA-positive case can come with biopsy report and PAP report at the same time. The sensitivity and specificity of both tests shall be updated on completion of the study.

\section{Abstract \#34: Novel Endocervical \& Ectocervical Spatula for Collection of Sample For Cervical Cytology}

Radhika N. Joshi ${ }^{1}$, Tanvee S. Kulkarni ${ }^{2}$

${ }^{1}$ Krishna Institute of Medical Science, Malkapur, Karad, Maharastra, India, ${ }^{2}$ Jeevan pathology laboratory, Khari corner, Kolhapur, Maharashtra, India.

Objectives: The cancer of uterine cervix is still the most prevalent cancer in rural India. Many group checkups and camps are regularly arranged in rural areas for early diagnosis, but still the sensitivity rate is very low for conventional pap smears. The most likely reason for this is the unsatisfactory rate due to widespread use of conventional Ayres spatula alone.

Methods: A novel spatula is devised to solve this problem keeping the cervical anatomy into consideration. The advantages of this new spatula are-

- No need for separate endocervical brush to take endocervical sample

- Spatula is wooden and gives good scrapes without trauma from all areas of cervix

- Customizable to small-, large- or irregular-sized cervix sizes

- It is economical \& reusable after high-level disinfection

Results: A project on cervical cancer screening at PHC was completed using this spatula with excellent results. A patent has been awarded to Krishna Institute of Medical Sciences, Karad from Indian Patent Office for this device on 25th November 2019.
Conclusion: This spatula can be an excellent alternative to conventional Ayres spatula and endocervical brush to take satisfactory pap smears in a resource-limited setting.

\section{Abstract \#42: Fluorescein sodium (FNa) during colposcopy-A study of diagnostic test accuracy}

Priya Bhati, Abraham Peedicayil, Rachel Chandy, Anitha Thomas, Ajit Sebastian, Vinotha Thomas

Christian medical college, Vellore, Tamil Nadu, India

Objectives: To assess diagnostic test characteristics of FNa compared to the definitive histopathological diagnosis of pre-malignant /malignant lesions of the cervix. To describe the correlation of FNa with VIA, VILI and colposcopy.

Methods: Seventy patients, with abnormal screening tests /symptoms, who were referred for colposcopy, were enrolled. In addition to the usual acetowhite and Lugol's iodine used in the colposcopy procedure, FNa was also used. Abnormal areas look fluorescent green when seen under UV light in a dark room. Biopsies were taken from the abnormal areas highlighted after staining and the histopathology report was the gold standard. For analysis, disease threshold for Biopsy was "CIN2 + ". For the index tests, positive result for acetowhitening taken as "distinct staining"; for lugol's iodine "distinct yellow" and for Swede score "score $>5$ ", and for FNa "distinct fluorescence".

Results: The sensitivity of FNa staining was 0.52 , lower than both acetowhite and lugol staining. The specificity was higher at 0.867 . Accuracy was 0.743 .

The prevalence of CIN2 + was 35.7. The PPV was 0.684 and, NPV was 0.765 . Positive likelihood ratio was 3.9 , and negative likelihood ratio was 0.55 .

The kappa coefficient for acetowhite and FNa was 0.538; and for Lugol's iodine and $\mathrm{FNa}$ was 0.54 ; both suggesting moderate agreement.

Conclusions: The sensitivity of fluorescent sodium was lower, but specificity was quite good with an accuracy of $74 \%$. FNa is low cost and easy to perform as well. It appears to have enough potential to deserve further study.

\section{Abstract \#61: Development of a urine preservation buffer for HPV DNA preservation}

Siddharth Anand, Shatakshi Ranade, Kunal Patil, Mridula Rani, Karthik Ganesan, Meenal Agarwal

Gene Path Diagnostics India Pvt Ltd, India

Objectives: The detection of human papillomavirus (HPV) DNA from urine can potentially increase the coverage of cervical cancer screening in women as it circumvents the need for cervical sampling. Urine, however, contains nucleases that degrade HPV DNA. Therefore, we aimed to develop a buffer that can preserve DNA in urine, thereby facilitating accurate and non-invasive detection of HPV.

Methods: HeLa cells were used to perform proof-of-principle experiments. DNA was extracted from urine spiked with a known number of HeLa cells with or without our buffer or commercially available urine preservation buffers stored at ambient temperature at different time points; appropriate controls were set up. Quantitative polymerase chain reactions (qPCRs) targeting a human housekeeping gene (RNaseP) and the L1 region of HPV were performed on the extracted DNA.

Results: The developed urine buffer protected DNA from degradation more effectively than did the commercial buffers. Additionally, the 
buffer enhanced DNA extraction even at the 0-day time-point. DNA in the control (urine $+\mathrm{HeLa}$ cells) degraded rapidly after day 3 , whereas the tube containing urine + HeLa cells + GPDx urine buffer showed a stable level of RNaseP over the 7 days.

Conclusions: The developed DNA preservation buffer offers a viable and cost-effective method for the storage and transport of urine at ambient temperatures. This is especially helpful in a developing country like India where there is a social stigma attached to cervical sampling. Noninvasive samples like urine along with appropriate HPV testing may help overcome logistical and social issues for cervical cancer screening programs.

\section{Abstract \#84: Challenges at designing of Easy Cervical Optimiser (ECO) device}

Shyamala Guruvare, Vidya Kudva, Keerthana Prasad

Kasturba Medical College, Manipal Academy of Higher Education, Manipal, India

Objectives: Recently, HPV testing is taking precedence in cervical screening, almost replacing the Papanicoloau smear. However, in resource-poor settings, visual inspection after acetic acid (VIA) still remains the screening method. The paper introduces a new device with inbuilt artificial intelligence (AI) system designed for cervical screening using VIA.

1. Description of the ECO device

2. Challenges faced during the project

Methods: The work on ECO device was funded by CAMTech India innovation project. The two main features of the device are 1. Image capturing and storing component 2 . Inbuilt image analysis and interpretation algorithm. The challenges faced during different phases of the project such as image acquisition, development of image analysis and interpretation algorithm are listed.

Results: The device: Image capturing application was optimized to have specified details of the screened woman, provision for capture before and after VIA. Analysis and interpretation application consisted of defining cervix region of interest after removing the specular reflections and a hybrid transfer learning technique for analysis. Setting the gold standard for comparison, obtaining varieties of VIA positive and negative images for training the algorithm, were the main challenges faced.

Conclusion: Details on how the challenges faced at designing ECO device were addressed are discussed.

\section{Abstract \#92: Never modalities under research for diagnosing early cervical cancer lesions}

Anshu Patodiya

Meera Hospital, Jaipur, India

Cervical cancer is one of its type of cancer where there is a long phase of pre-invasive stage and an easy approach to screening. Inspite of these facts, we have yet to find out a test which can be easily done, widely available with high sensitivity and cheap.

Till now Paps, VIA \& VILLI are being used to detect and look for changes associated with pre-malignant leisons of cervix. Because of cost requirement, trained staff and poor sensitivity, we are missing out on opportunities to screen women more so in rural areas.

This presentation is to bring to your notice an Artificial Intelligence System being developed that can outperform human experts in identifying Cervical Pre-Cancer called automated visual evaluation.
Abstract \#99: Can field workers be trained to use transvaginal colposcope for point of care diagnosis \& management of precancerous cervical lesions

Jayashree Natarajan, Nimmi Ramanujam, Marlee Krieger, Shachi Vashist, Swati Tomar, Neerja Bhatla

All India Institute of Medical Sciences, New Delhi, India

Objectives: Screening programs in low-middle-income countries are not able to reach the target population. Portable colposcopes can decrease costs-cum-referral rates. To compare the quality of images captured with a transvaginal colposcope (TVC) by a field worker $(\mathrm{FW})$, and to assess the image adequacy for determination of colposcopic diagnosis and management decisions.

Methods: Women aged 21-65 years with abnormal screening report/ cervical symptoms were enrolled. FW reported visual inspection with acetic acid (VIA) test results, captured images with TVC. The physician performed cervical assessment with colposcopy by both TVC and Digital video colposcope, calculated Swede scores, and obtained biopsy from all lesions. Images captured by the FW were assessed by colposcopist for adequacy for decision-making and management. Next, the ability of the FW to identify patients for referral was assessed, agreement between the Swede score from both sets of images was calculated.

Results: Among 125 subjects, 96 (76.8\%) were VIA positive and 12 $(9.6 \%)$ had CIN 2 + lesions. Of the images captured by the FW 122 $(97.6 \%)$ were adequate for interpretation. Swede score calculated from the images captured by both FW and physician showed perfect agreement $(\mathrm{K}=1.0)$. At Swede score cut-off of $>5$ to detect CIN2 +, images collected by both had sensitivity, specificity, PPV and NPV of $83.3 \%, 73.3 \%, 30.3 \%$ and $97.4 \%$, respectively. Images were reviewed by FW and colposcopist. FW and colposcopist estimated that 98 (78.4\%) and $34(27.2 \%)$ subjects need referral.

Conclusions: TVC can be used by FW to capture cervical images which can be reviewed by colposcopist to reduce referral rates.

\section{Abstract \#102: To Evaluate the role of P16ink4a immunostaining} in VIA screen positive women for detection of CIN2 +

\section{LalitaVerma, Saritha Shamsunder}

Department of Obstetrics and Gynaecology, Vardhman Mahavir Medical College and Safdarjung hospital, New Delhi, India

Objectives: To evaluate the role of p16Ink4a immunostaining for detection of CIN $2+$ in women who had a positive screening test using visual inspection with acetic acid (VIA).

Methods: Opportunistic screening of women (30-50 years) coming to gynecology clinic by VIA was performed; the screen positive women were included in the study which had the IRB approval. A cytology slide for p16 Ink4a immunostaining and biopsy were then performed sequentially. The sensitivity, specificity, PPV \& NPV of p16 Ink4a immunocytochemistry was evaluated with histopathology as the gold standard.

Results: p16 Ink4a positivity showed a linear correlation with increasing grade of CIN. p16 Ink4a positivity was seen in $6 \%$ of CIN $1,80 \%$ of CIN $2,100 \%$ of CIN 3 and SCC. The sensitivity and specificity of p16 Ink4a immunocytochemistry for detecting CIN 2 or more was $87.5 \% \quad(95 \% \mathrm{CI} \quad 61.65-98.45)$ and $97.06 \% \quad(95 \% \mathrm{CI}$ 84.67-99.93).

Conclusion: With high sensitivity and specificity, p16Ink4a immunocytochemistry could be a viable option for triaging VIA positive women. 
Abstract \#134: A Pilot Project: Agreement Level of Direct VIA, 8 Megapixels Smartphone Camera, and 12 Megapixels Smartphone Camera

Tania Chandra, Mohammad Nailul, Fahmi Yodi, Mahendra Dhata

Faculty of Medicine, Public Health, and Nursing-Universitas Gadjah Mada, Indonesia

Objectives: To measure the agreement level between direct and indirect interpretation of VIA test using digital image taken with smartphone camera for cervical cancer screening.

Methods: 208 women aged 20-60 years who attended the cervical cancer screening program held by Jogjakarta Health Department in Sanden Primary Health Center, Indonesia, agreed to participate in this study. The screening was done by applying acetic acid to the cervix (VIA). After application, about 1-2 min (s), the absence or appearance of acetowhite lesion was observed. Images of the cervix then taken using 8 megapixels and 12 megapixels smartphone cameras. Direct interpretation of VIA test was done at the screening location, while indirect interpretation was done away from the screening location by 3 experienced obstetrics and gyncecology doctors through the digital images taken with the smartphone cameras. Agreement level between direct and indirect VIA interpretation was measured. Furthermore, agreement level between 8 and 12 megapixels camera and agreement among raters who did the indirect VIA interpretation was also measured.

Results: Agreement levels between direct and indirect VIA interpretation are 0.303 (95\%CI: $0.063-0.542)$ and 0.471 (95\%CI: 0.193-0.748) for 8 megapixels and 12 megapixels camera consecutively. Agreement level between 8 megapixels camera and 12 megapixels camera is 0.580 (95\%CI: 0.336-0.824). The inter-rater agreement levels are 0.408 (95\%CI: $0.243-0.571)$ and 0.293 (95\%CI: 0.070-0.502) for 8 megapixels and 12 megapixels camera consecutively.

Conclusions: Smartphone camera is may increase the efficiency of VIA test for cervical cancer screening.

\section{Abstract \#138: Feasibility and Triage Study of HPV Genotyping of Self-Sampling in Cervical Cancer Screening on Internet-Based in China}

LiJingran Wu, Lihui Wei, Zhijun Zhang

Peking University People's Hospital, China

Objectives: To evaluate the feasibility of vaginal self-sampling for cervical cancer screening on Internet-based, and the triage value of high-risk HPV genotyping.

Methods: Totally 10,056 women aged $30 \sim 59$ years with selfsampling were recruited for cervical cancer screening between September 2018 and May 2019 in 8 provinces, and 984 had histological diagnosis. The 14-HPV testing was performed by real-time PCR assay. Women registered online (http://47.106.227.241/) and signed online informed consent. Self-sampling by themselves from the vagina. The feeling and acceptability of self-sampling were investigated after sampling.

Results: Of the 10,056 attenders, the success rate of self-sampling was $99.86 \%$. HPV infection rate is $15.56 \%(1565 / 10,056)$ among 8 provinces. The positive proportion of the top $5 \mathrm{HPV}$ genotypes were HPV52, $-16,-58,-39,-56$. Among the $14 \mathrm{hrHPV}$ involving HSIL + cases, HPV16, $-33,-52,-58$ were most likely to develop HSIL + . If HPV16/18 combined with HPV33, detection rate of HSIL + increased by $52.3 \%$, colposcopy referral only increased by $4.2 \%$. In addition, $92.3 \%$ women feel that it is easy to master selfsampling, $86.4 \%$ have no discomfort, and $60.8 \%$ attenders are more likely to choose self-sampling for cervical cancer in the future.
Conclusions: The self-sampling cervical cancer screening on internet-based is a simple, convenient and very promising screening model with a more higher acceptability of self-sampling in the future in China. Extensive genotyping could identify some more high-risk genotypes for detecting HSIL + , apart from HPV 16/18, HPV33 showed a higher prevalence in detection of HSIL + lesions, combine with 3 types may be as a triage way.

\section{Abstract \#195: Are Novel Methods Of Cancer Cervix Screening Prudential? A Literature Review}

\section{Sufia Athar}

Department of Obstetrics and Gynecology, Al-Wakra Hospital, Qatar

Objective: Comparative analysis of diagnostic accuracy and costeffectiveness of conventional and novel methods of cancer cervix screening.

Design: A literature review.

Method: An internet-based literature search for all the available methods of cancer cervix screening in the PubMed/MEDLINE, Scopus, Cochrane, and EMBASE databases. Studies published between 1990-2020 were evaluated. 52 studies were included. Relevance screening, data extraction, risk of bias analysis, and quality assessments were conducted. The sensitivity and specificity of screening methods and their cost-effectiveness were evaluated.

Results: In comparison to conventional methods (pap smear, HPV DNA, VIA, VILI), Care HPV assays and Hybrid Capture 2 test have higher sensitivity $91.1 \%$ and specificity $83.7 \%$. They provide logistical and economic advantages but lack sensitivity as compared to HPV DNA. Detection of TERC predicted progression of CIN1/2 to CIN3 after a follow-up of two months to three years with $100 \%$ sensitivity and $70 \%$ specificity. Cost and need for technical skills are the limitations. The sensitivity and specificity of p16/Ki-67 dualstaining for CIN2 + were $74.9 \%-90.9 \%$ and $72.1 \%-95.2 \%$, respectively. This method is useful in the unnecessary follow-up of HPV positive and is cost-effective for HSIL. Optical methods of screening are emerging as non-invasive tests with high sensitivity $90-98 \%$ but low specificity for HSIL lesions. They may be considered as the firstline screening and triage during the same medical visit, thus, significantly reducing the cost.

Conclusion: The novel methods are cost-effective in women with HSIL and those who are HPV positive with better sensitivity as compared to the conventional methods.

\section{Abstract \#224: Pap Smears to Digital Darwinism}

\section{Puneet Chandna}

MD and Board member, Ace Probe Technologies India Pvt Limited

It should not come as a surprise that technology, despite the dramatic way in which it has altered our ability to image the body, measure and monitor its molecular structure, can also fail just as badly as humans fail. The Fourth Industrial Age cantered on artificial intelligence (AI), robotics, and Big Data, ushers in a profound transition that can already be seen in how we live and function, and maybe even in how we think about ourselves as humans. It has a lot of potential not only to support, but also to hurt, by exacerbating the deep divide that already exists between those who have a lot and those who have less with each passing year. It should not come as a surprise that technology, despite the dramatic way in which it has altered our ability to image the body, measure and monitor its molecular structure, can also fail just as badly as humans fail. 
ARTIFICIAL INTELLIGENCE in medicine is expected to provide integrated, panoramic views of individuals' medical data to avoid mistakes; to aid in treatment; and treatment decisions and interpretations; and tests; and to minimize misdiagnoses and falsepositive results. Useful[preoperative data]intelligence from colposcopy for preoperative data, the population has been segmented into three categories: Extreme Dysplasia, Cervical Intraepithelial Carcinoma (CIC), and Invasive Cervical Cancer (IC). Methods for applying deep learning to colposcopy image classification may be preliminary, but it suggests that clinicians and researchers who are not specialists in artificial intelligence or machine learning can make use of deep learning.

AI will be used as a promising tool in gynaecology and obstetrics to solve a number of longstanding challenges; and AI could still serve as a means to increase knowledge and help clinicians to make decisions in a range of fields in obstetrics and gynaecology.

\section{Abstract \#242: A Novel Approach In Cervical Cancer Screening N. Hephzibah Kirubamani}

Saveetha Medical College, Mylapore, Chennai, India

Objective: Fluorescence spectroscopy measures fluorophores which might change according to pre-neoplastic and neoplastic tissues. The aim was to assess performance of fluorescence spectroscopy in the diagnosis of different grade of squamous intraepithelial cervical lesions (SIL). The objectives were to compare fluorescence spectroscopy findings with colposcopic findings, Papanicolaou smear and histopathology report.

Method: Prospective observational study was conducted after ethical clearance and informed consent. 65 women between 25 to 60 years attending the outpatient clinic of Saveetha Medical College Gynaecology Department between June2019to Dec 2019 with discharge per vaginum, low backache, irregular bleeding and post-coital bleeding were included. After gynaecological workup, Pap smear and colposcopy and. cervical biopsy were done. Fresh tissue was sent for Fluorescence spectroscopy. Tissue was excited using a filtered shortarc xenon lamp at 375-460 nm and a power output of $200 \mathrm{~mW}$. Spectral measurements were performed. Same biopsy tissue was confirmed by the final histopathological diagnosis. Changes in the fluorescence properties of cervical squamous epithelium that are associated with the development of neoplastic disease were assessed. Results: At $380 \mathrm{~nm}$ excitation of the tissue, high SILs can be differentiated from low SILs with sensitivity of $79 \%$ specificity $74 \%$, PPV $81 \%$, respectively. At $460 \mathrm{~nm}$ excitation, low SILs can be differentiated from normal with an average sensitivity, specificity and positive predictive value of $82 \%, 78 \%$ and $74 \%$, respectively, which is similar to that of colposcopy. There was $100 \%$ correlation of fluoroscopy report with HPE.

Conclusion: Spectroscopic methods are potentially a reliable, fast method and can be an alternative to other screening methods. Spectroscopic signatures can be used for diagnosing SILs with a handy device.

\section{Abstract \#253: PAX-1 Methylation Levels in Cervical Scrapings as Diagnostic Biomarker in Pre-invasive and Invasive Cervical Cancer}

Bhavya Prasad $^{1}$, Shalini Rajaram ${ }^{1}$, Bindiya Gupta ${ }^{1}$, B.D. Banerjee ${ }^{2}$, V.K. Arora ${ }^{3}$, Sandhya Jain ${ }^{1}$

${ }^{1}$ Department of Obstetrics and Gynaecology, ${ }^{2}$ Department of Biochemistry, ${ }^{3}$ Department of pathology, University College of Medical Sciences and GTB Hospital, Delhi, India
Objective: The objective of the study was to quantify and compare the levels of methylated PAX1 gene in normal, cervical intraepithelial neoplasia2/3 and invasive cancer.

Design: Observational analytical study.

Method: In this experimental study 201 screen positive women (VIA, VILI and Pap test) underwent colposcopy and 94 women with Swede score of 5 or more were biopsied. On histopathology 55 had chronic cervicitis or squamous metaplasia, 13 had diagnosis of CIN 1, 20 had CIN $2 / 3$ and 6 had invasive squamous cell carcinoma. 14 women with obvious growth were also recruited. Cervical scrapes taken, were sent for PAX 1 methylation level. 60 women were recruited to the study and allocated into three groups, i.e. Benign cervices (Group1; $\mathrm{n}=20$ ), CIN 2/3 (Group 2; $\mathrm{n}=20$ ) and invasive cervical carcinoma (Group; $\mathrm{n}=20$ ).

Results: The mean PAX1 methylation levels in benign lesions, CIN $2 / 3$ and invasive cancer were $9.58 \%(\mathrm{SD} \pm 2.37 \%), 18.21 \%(\mathrm{SD} \pm$ $2.67 \%$ ) and $24.34 \%$ (SD $\pm 4.09 \%$ ), respectively, with $\mathrm{p}$ value of $<0.001$. The ROC curve for analysis of PAX 1 methylation percentage in cancer versus CIN 2/3 showed the best cut-off point was $21.8 \%$ (sensitivity $=76.67 \%$ and specificity $=100 \%$. $(\mathrm{P}<0.001)$, while analysis in CIN 2/3 versus normal showed the best cut-off point as $12.38 \%$ ( sensitivity and specificity $=100 \%$ ).

Conclusions: PAX 1 methylation levels have a promising role in identifying CIN2 and worse lesions. Addition of DNA methylation testing in screening programs will need validation with larger multicentric trials.

\section{Abstract \#312: Digital and Artificial intelligence-based examination for cervical abnormalities using SmartScope®}

Veena Rahatgaonkar-Joshi, Vijay Nidhi Dhasmana

Department of Obstetrics and Gynaecology, Deenanath Mangeshkar Hospital and Research Centre, Erandwane, Pune, India

Objective: Feasibility assessment of Smart Scope ${ }^{\circledR}$ (SS) coupled with Artificial intelligence-based assessment (AI-A) as a potential cervical cancer screening method.

Design: Observational, single arm study conducted at Deenanath Mangeshkar Hospital, Pune, India.

Method: Study was conducted in two phases; 509 in first and 246 women in second phase were enrolled. In the first phase, naked eye visual screening (NE), cytology and SS aided visual assessment (SS$\mathrm{VA}$ ) of cervix for detection of precancer and cancer were compared considering histopathology as gold standard. In the second pilot phase, a set of cervical images captured by SS was assessed (blinded) by expert health provider as well as by AI-A and were compared with histopathology as gold standard. The AI assessed cervical images in 3 categories; Green (normal), Amber (benign) and Red (precancer and cancer).

Result: In first phase, SS-VA demonstrated $100 \%$ sensitivity and NPV. In second phase, SS-VA by an expert could detect all confirmed precancer cases but over-estimated the lesions in 8 women. AI-A underestimated one precancerous lesion as benign but reduced the overestimation of precancerous cases.

Conclusion: SS-VA has great potential as a primary screening test in a low-resource setting due to its better sensitivity and NPV. The AIbased assessment for detection of cervical abnormalities may help the health providers for immediate risk classification of cervical abnormalities and reduce burden on tertiary centers for colposcopy. This simple single visit test if used widely may help to increase the participation of women in screening program. 


\section{Colposcopy}

Abstract \#3: Correlation of Swede and Reid Colposcopic Indices for Predicting Premalignant Lesions of Cervix

S Murali, Avantika Gupta, Arthi Thangavel

Department of Obstetrics and Gynaecology, JIPMER, India

Objectives: To evaluate the efficacy of Swede and Reid Colposcopic Indices to predict high-grade lesion of cervix and to assess the strength of correlation between these two Indices.

Methods: In this retrospective study, 160 women aged 30 to 69 years with abnormal screening results were included. In all women who underwent colposcopy, both Reid Colposcopic index and Swede index were routinely calculated in our clinic and biopsy taken from all abnormal areas. Performances of both the scores were calculated and compared using our colposcopy register data.

Results: A total of 49 (30.6\%) CIN 2 + lesions were detected. Reid Colposcopic index at a cutoff of 5 had sensitivity, specificity, positive predictive value, and negative predictive value for detecting CIN2 + lesions of 95.9\%, 93.7\%, 87\%, and 98.1\%, respectively. Using Swede index at a cutoff of 8 , sensitivity, specificity, positive predictive value, and negative predictive value were $44.9 \%, 100 \%$, $100 \%$, and $80.43 \%$, and with a cutoff of 5 , these were $100 \%, 85.6 \%$, $75.38 \%$, and $100 \%$, respectively. The correlation coefficient (R2) was 0.91 by Spearman rank correlation coefficient.

Conclusion: Swede index has a good correlation with Reid Colposcopic index. Swede score of 8 or more has $100 \%$ specificity and can be used for screening-and-treating in the same setting to decrease the overtreatment rate.

\section{Abstract \#52: An audit on Colposcopy at a tertiary teaching hospital in South India}

AnithaThomas, Ajit Sebastian, Dhanya Susan Thomas, Rachel George Chandy, Priya Bhati, Grace Rebekah

Department of Gynaecologic Oncology, Christian Medical College, Vellore, India

Objectives: To audit the performance of colposcopy with respect to indications, results and its correlation with post-procedural biopsies using the NHS Cervical Screening Programme Colposcopy and Programme guidelines in collaboration with the British Society for Colposcopy and Cervical Pathology.

Methods: We audited data of colposcopies done for abnormal cytology smears from November 2017-June 2018. Indications for the colposcopy, findings, biopsy results, follow-up procedures done were looked into. These were then analysed according to the International federation for colposcopy/ NHS SPCP guidelines and checked for the fulfilment of criteria.

Results: A total of 14,332 Pap smears were done at this tertiary hospital during this period. Colposcopies were done for 150 of these patients. Of these, 67 cases on whom colposcopies done for the highrisk HPV positive and Pap smear abnormality ASCUS and above were audited. The audit revealed compliance in 7 out of 8 criteria except for in the adequacy of the reporting of colposcopy of the transformation zone not achieved in $2.5 \%$. The correlation for the low-grade score $<6$ to low-grade biopsies $(<$ CIN II) and the highgrade Swedes score $>6$ to higher grade lesions was good with kappa coefficient of 0.572 and $p$ value of $<0.000$.

Conclusions: Audit of colposcopy performance using the NHSCSP guidelines revealed that compliance to guidelines was good. Correlation of colposcopy findings with the diagnostic biopsy was good. Supervised training is essential and can lead to quality improvement with fulfilment of all standards.
Abstract \#66: Evaluation of colposcopic Swede score in the detection of Cervical Intraepithelial Neoplasia

Vishakha Ahuja, Nandita Maitra

Shree Sayaji General (SSG) Hospital, Vadodara, India

Objectives: To evaluate colposcopic Swede Score in the detection of Cervical Intra-epithelial Neoplasia.

Methods: An observational study where 50 Women coming to gynecology OPD of our hospital with complaints of abnormal uterine bleeding, chronic pelvic pain, vaginal discharge, abnormal Pap smear, acetowhite lesion on application of acetic acid (VIA positive), HPV positive, were examined using digital video colposcope colpro 222. Colposcopic guided punch biopsies were taken from abnormal areas and sent for HPE examination. The swede scores were compared against the histologic diagnosis. Data and images were stored using the Colpro software. Diagnostic accuracy of swede score in the detection of cervical neoplasia would be calculated using appropriate tests.

Results: Agreement between colposcopic swede score and histopathology. Biopsy detected CIN-1 in 17 (34\%) patients CIN2 + (CIN 2, CIN 3) in $8(16 \%)$ patients, $2(0.04 \%)$ women were found to have carcinoma in situ. 17 women were positive for HPV, 3 women had HPV 16, 14 women had other high-risk HPV, which are independent risk factors for CIN.

Conclusion: Colposcopy by Swede score could offer a more accurate screening and selection for immediate treatment of cervical lesions in low-resource settings.

\section{Abstract \#72: See with Colposcope (primary screening test) and Treat with LEEP (All CIN) at SEE \& TREAT Colposcopy Clinic}

\section{Sandip Phadke}

Phadke Hospital\& District Womens Hospital, Akola, Maharashtra, India

Objectives: To Screen Women from Local Population with Colposcope as a Primary Screening method \& Treat with LEEP whenever any grade of CIN is documented.

Methods: Of Total 1200 Women who were Screened at SEE \& TREAT Colposcopy Clinic; 97 Women diagnosed with varoius grades of CIN Lesions were treated with LEEP. All treated Women were followed up with colposcopy at 6 months and 12 months.

Results: At 6 months, 10 women lost to follow-up. At 12 month, we lost follow-up of 30 women. Healing of cervix was remarkable with no appearance of CIN lesions in all women we followed up at 6 \& 12 months complications during LEEP and during follow-up if any noted. All LEEP Specimens were sent for HPE and correlation with colposcopic findings done.

Conclusions: (1) Colposcopy can be used by trained Gynaecologists for primary screening of women for cervical cancer (2) LEEP can Safely be done at SEE \& TREAT Colposcopy Clinic as a OPD Procedure. (3) LEEP is an effective treatment modality for all grades of CIN. (4) See with colposcope (as a primary screening test for detection of cervical precancerous lesion where Lab. facilities for Cytology are not up to the mark and HPV DNA tests are either lacking or costly) \& treat all CIN with LEEP as OPD procedure (where compliance of women for follow-up is doubtful), can be a good strategy to reduce the incidence of cancer cervix. 
Abstract \#78: Colposcopic evaluation of cervix in abnormal Pap smear: A prospective study at tertiary care centre

Dipali Prasad, Archana Sinha

Department of Obstetrics \& Gynecology, IGIMS, Patna, India

Objectives: In India, cervical cancer is the second most common cancer in women (aged 15-44 years) after breast cancer accounting for almost $14 \%$ of all female cancer cases. Objectives were to correlate the findings of Pap smear with colposcopy and to assess the utility of colposcopy in detecting the premalignant and malignant lesions of cervix.

Methods: This was a prospective observational study of 150 symptomatic women attending the Gynecology OPD of Indira Gandhi Institute of Medical Sciences, Patna from July 2019 to June 2020. Evaluation of all symptomatic women done with Pap smear, colposcopy and biopsy in selected cases.

Results: Commonest complaint was white discharge per vaginum in $50 \%$ followed by pelvic pain in $20 \%$ women. $9 \%$ women had abnormal pap smear findings with $2.6 \%$ of women had ASCUS, $0 \%$ had ASC-H, 3.3\% had LSIL and 3.3\% had HSIL, 68\% had inflammatory and $22 \%$ with normal smear. $30.6 \%$ had abnormal colposcopy with maximum, and $15.3 \%$ women had acetowhite lesions. Sensitivity of pap smear was $50 \%$, while sensitivity of colposcopy was $83.3 \%$. Conclusions: Pap smear had poor sensitivity as compared to colposcopy. Hence simultaneous use of colposcopy has shown to increase in the rate of carcinoma cervix detection in symptomatic women.

\section{Abstract \#80: The Role of Colposcopy in Determining Cervical Cancer Risk in Negative for Intraepithelial Lesion or Malignancy/ High-Risk HPV-Positive Women}

\author{
Jennifer Varughese \\ Northwell Phelps Hospital, United States
}

Objectives: According to ASCCP guidelines, in women above 30 years with cytology negative, human papillomavirus (HPV) positive, it is acceptable to either repeat co-testing in 1 year or perform colposcopy for further evaluation. This choice of recommending colposcopy or follow-up co-testing is determined by the provider. Hispanic women have the highest incidence of cervical cancer compared to other races and there has been minimal research on the benefits of performing a colposcopy in a higher risk population to evaluate for grade 2 or higher cervical intraepithelial neoplasia.

Methods: Retrospective study included 600 Hispanic women over 30 years with negative intraepithelial lesion or malignancy, positive high-risk HPV across six different Open Door Family Medical Centers from October 2014 to January 2019. These women who tested NILM, positive high-risk HPV were recommended to receive a colposcopy or follow-up in 1 year for co-testing. These results were then analyzed to determine the incidence of grade 2 or higher cervical intraepithelial neoplasia.

Results: Among women with NILM/ + HRHPV, 235 (39\%) had colposcopy. Of those who received a colposcopy, $82 \%$ had benign pathology and $<3 \%$ had grade 2 or higher CIN. At subsequent screening exams, $29 \%$ of women had negative cytology/negative HPV.

Conclusion: Colposcopy after a NILM/ + HR HPV screening result did not lead to earlier detection of grade 2 or 3 cervical intraepithelial neoplasia. This result indicates that women of higher-risk population, NILM/ + HRHPV, may benefit from annual co-testing and if any cytologic changes present, consider follow-up with colposcopy.
Abstract \#93: Agreement between Colposcopic Diagnosis and Cervical Histology when Colposcopy is Performed on Hybrid Capture 2 Positive Women

Puja Chatterjee, Dipanwita Banerjee, Ranajit Mandal, Manisha Vernekar

Chittaranjan National Cancer Institute, Kolkata, India

Objectives: Colposcopy is a subjective method of diagnosing cervical neoplasias. Earlier studies have evaluated correlation between colposcopy and histopathology in settings where colposcopy was done on women referred for abnormal cytology report. We aimed to determine the agreement between colposcopy and histology diagnosis when colposcopy is performed for Hybrid Capture 2 (HC 2) positive women.

Methods: Women between 30 to 60 years were screened for cervical cancer by $\mathrm{HC} 2$ in a community-based demonstration program. The screen positive women had colposcopy. Cervical punch biopsies were obtained from all the women. Both colposcopy and histology diagnosis were categorised as normal, HPV changes, LSIL, HSIL and cancer. Agreement was calculated by weighted kappa. Study had Institute Ethics Committee approval.

Results: A total of 689 women had colposcopy due to HC 2-positive results. On histology of 634 samples, there were 79 cases of LSIL, 30 cases of HSIL and 5 cases of cancer in the total cohort. Rest of the women were normal or had HPV changes. There was agreement in $78.65 \%$ of the observations. The weighted Kappa value of $0.275(95 \%$ C.I. 0.194 to 0.356 indicates fair strength of agreement.

Conclusion: When colposcopy is performed on women referred due to positive $\mathrm{HC} 2$, the agreement is fair between colposcopy and histology diagnosis. The study is ongoing and updated results will be presented.

\section{Abstract \#101: Correlation of Reid's Colposcopic Index and Swede Score with Cervical Histopathology for diagnosing Cervical Lesions}

Shuchi Jain

Institute of Medical Sciences, BHU, India

Objectives: 1 . To find correlation between Reid's Colposcopic Index (RCI) and Swede Score for predicting cervical lesions 2. To find accuracy of prediction of RCI score and Swede Score with Histopathology of cervical lesions.

Methods: A cross-sectional study of 100 patients with cervical lesions, aged 30-60 years, underwent colposcopy. RCI and Swede score were determined and colposcopy-directed biopsy from abnormal areas was done, and in case no such areas were delineated, then four-quadrant biopsy was taken from cervix and sent for histopathological examination (HPE).

Results: $39 \%$ of the patients were in the age group of 30-40 years. RCI score with cut off value $\geq 5$ with respect to HPE showed maximum sensitivity $88.2 \%$, specificity $85.5 \%$, NPV $97.3 \%$ ( $\mathrm{p}<0.001$ ) for SCC and for CIN III sensitivity $82 \%$, specificity $80 \%$, NPV $97.3 \%$ ( $p<0.001)$. Swede Score cut off value $\geq 8$ with respect to HPE showed maximum sensitivity $100 \%$, specificity $86.7 \%$, PPV $60.7 \%$, NPV $100 \%(\mathrm{p}<0.001)$ for SCC and for CIN III sensitivity $63.6 \%$, specificity $76.4 \%$, NPV $94.4 \%$ (p 0.02 ). Good correlation was found between the Reid's score and Swede score; Spearman's correlation coefficient $\mathrm{r}=0.804 ; \mathrm{p}<0.001 ; \mathrm{r} 2=0.64$.

Conclusion: RCI core $\geq 5$ or Swede score $\geq 8$ can be used for performing direct excisional procedure as a "see and treat" method. 
Abstract \#107: Correlation of cervical cytology and Colposcopy taking Histopathological as gold standard in Detection of Premalignant Lesions of Cervix

Mayank Jain, Pratibha Singh, Garima Yadav, Meenakshi Gothwal, Navdeep Ghuman, Poonam Elhence

Department of Obstetrics and Gynaecology, AIIMS, Jodhpur, India.

\section{Objectives:}

1. To find correlation in cervical cytology, and colposcopy in diagnosing premalignant lesions of cervix

2. To find correlation in colposcopy and histopathology in detection of premalignant lesion of cervix

Methods: The study is a prospective observational study planned for 2 years; interim results obtained over a period of 1 year are discussed. The study is being conducted at Department of Obstetrics \& Gynecology at AIIMS Jodhpur.

Inclusion criteria: screen positive women undergoing colposcopy and biopsy. Age between 21-70 years.

Exclusion criteria: Pregnant women, women who did not have colposcopy or cervical biopsy.

These cases were analysed and sensitivity and specificity in detecting premalignant lesion was calculated.

Results: Good correlation was observed between colposcopy and histopathology with a sensitivity of $82 \%$ and specificity of $98 \%$.

Cytology did not correlate well with colposcopy [specificity of 58\%] and histopathology [specificity of $65 \%$ ].

Conclusion: Cervical cytology did not co-related well with colposcopy and histopathology, while good correlation was observed between colposcopy and histopathology for detecting premalignant lesions of cervix.

\section{Abstract \#118: A Retrospective Analysis: Evaluation of Unhealthy Cervix and Correlation of Colposcopy and Papanicolau Smear in Cervical Malignancy Screening.}

\section{Srishti Prakash, Seema Prakash}

Department of Obstetrics and Gynaecology, VMMC and Safdarjung Hospital, Delhi, India

Objectives: To analyse correlation between Colposcopy and Papanicolau smear in cervical Cancer screening in patients with unhealthy cervix.

Methods: A retrospective analysis was done at GTB Hospital, Delhi, India from January 2019 to December 2019 on 120 women with unhealthy cervix. Patients with unhealthy cervix on naked eye examination were analysed who underwent Pap smear followed by colposcopy and colposcopy-directed biopsy. Papanicolau smear evaluation of unhealthy cervix is routinely done followed by Colposcopic evaluation. Green filter, Acetic acid, Lugols Iodine are used to assess the cervix. Any abnormality is identified, lesion labelled, colposcope-directed biopsy of cervix is taken and Histopathology of biopsy is done.

Results: Analysis of 120 women who underwent colposcopy following a clinical examination and Pap smear was done. Of the 120 patients, $40 \%$ were in the age group $31-50$ years and $20 \%$ were in the age group of $51-65$ years. $80 \%$ of patients were more than Para- 2 . Cervical biopsy were taken in $34 \%$ of cases in whom either Pap smear or colposcopy or both were abnormal and their correlation was studied. $15 \%$ patients with normal PAPs had CIN-I and none had CIN-III on Histopathological examination. In $2 \%$ cases with HSIL had CIN-III and $8 \%$ with LSIL had CIN-II.
Conclusions: Cervical cancer is the commonest malignancy in India and third most common cancer in the world. Both Pap smear and colposcopy can be used reliably to screen women having abnormal symptoms for diagnosing pre-malignant lesions. Histopathology of suspected lesion remains gold-standard for final diagnosis of precancerous lesions.

\section{Abstract \#121: Colposcopy in clinically suspicious cervix: A study} from eastern state of India

Kavya Abhilashi, Sangeeta Pankaj, Jyotsna Rani, Satya Kumari, Pratibha Kumari

IGIMS, Patna, India

Objectives: Suspicious-looking cervix includes all cervical lesions having potential for cervical cancer. Mostly it includes benign and inflammatory conditions of cervix like ectropian, polyp, leukoplakia and nabothian cyst and patient may be asymptomatic or have complaints of abnormal vaginal discharge and post-coital bleeding. To evaluate the colposcopic outcome of women with clinically suspicious cervix.

Methods: A prospective cohort study of women with clinically suspicious cervix referred for colposcopy was conducted. Such patients underwent screening by pap smear \& colposcopy along with optional HPV testing. Relevant data were collected and analyzed using appropriate statistical method.

Results: A total of 313 patients were included in the study. Amongst women $(240 / 313)$ with negative primary screening only 6 out of 147 who underwent biopsy were reported CIN I/ CIN II on histology and rest had chronic cervicitis. CIN III \& invasive lesions were found in women with abnormal pap and positive for co-testing report. Positive predictive value of colposcopy for detecting CIN \& invasive one was higher but had poor specificity and was observer dependent.

Conclusion: Clinically suspicious cervix should be triaged by primary screening method, either cytology or co-testing. Colposcopy should be suggested for women with positive primary screening test.

\section{Abstract \#154: A Boon-Unrecognized}

Jeena Baburaj

Nalupurakkal, Narayana Nagar, India

Objectives: To study the applicability of Office colposcopy as a part of standard gynaecological examination in Kerala.

Methods: This Colposcopic study was conducted in 1000 women between 30 to $60 \mathrm{yrs}$. They came to my outpatient clinic at Vatakara, Kozhikode district, Kerala, with various gynecological complaints. Study duration was 5 years from 1/6/2011. The importance of cervical screening was discussed with all married gynaecological patients. 1000 subjects agreed for colposcopy examination. Office colposcopy was done using ASCON AC 3 with a fixed magnification of $6 x$.

Results: Colposcopy study was adequate in 897 (89.7\%) subjects and inadequate in $105(10.5 \%)$. Normal colposcopic findings were seen in 254 women $(25.4 \%)$. Colposcopic diagnoses of precancerous lesions were made in 209 (20.9\%) subjects. Out of this LSIL was diagnosed in $193(19.3 \%)$ and HSIL was seen in $16(1.6 \%)$. Invasive carcinoma was diagnosed in $5(0.5 \%) .34 .4 \%$ (344) of subjects had benign conditions of cervix \{c/c Cervicitis 187 (18.7\%), Severe ectropion 96 $(9.6 \%)$, cervical polyps $61(6.1 \%)\}$. The incidence of infection was $18.8 \%$ (188) candida infection $10.3 \%$ (103), trichomoniasis $6.1 \%(61)$, mixed bacterial infection $24(2.4 \%)$. 
Conclusions: In a state like Kerala where people are educated and health conscious, unfortunately, we have no organized screening program for cervical cancer. If office Colposcopy is accepted as a part of standard gynaecological examination, every gynaecologist can diagnose cervical pathology easily and can give necessary treatment in the same sitting. Normal Colposcopy findings, confidently predicts cancer-free cervix for the next 5-10yrs.

\section{Abstract \#180: Diagnostic Accuracy of Colposcopy-Directed Cervical Biopsy in the Management of Women with Cytologic High-Grade Squamous Intraepithelial Lesions}

\section{Anjana J S, Rema P, Suchetha S Dr Siva Ranjith J Dr Dhanya D}

Regional cancer centre, Medical college Kumarapuram road Medical college campus Chalakkuzhi Thiruvananthapuram Kerala, India

Objectives: To compare the histological features, recurrence rate and survival, between different histological types, in patients who underwent radical hysterectomy $(\mathrm{RH})$ for early cervical cancer.

Design: Retrospective study.

Methods: This study compared the outcome of patients with squamous cell carcinoma (SCC) and adenocarcinoma cervix (AC) who underwent RH at our institute from $1^{\text {st }}$ January 2007 to $31^{\text {st }}$ December 2018. Patient details were collected from medical records. Kaplan Meir survival curves were generated to assess disease-free survival (DFS) and overall survival (OS).

Results: A total of 136 patients with early cervical cancer underwent RH during this time period. 95 patients $(69.8 \%)$ had SCC and 41 patients $(30.1 \%)$ had AC. The baseline patient profile, preoperative FIGO stage were comparable between the two groups. The number of patients having tumors size greater than $4 \mathrm{~cm}$ was higher in case of AC compared to SCC (19.5\% vs $2.2 \%$, p value 0.002$)$. The rate of parametrial involvement and nodal metastasis were comparable. Significantly higher percentage of patients with $\mathrm{AC}$ received adjuvant therapy compared to SCC $(75.6 \%$ vs $53.9 \%$, p value 0.021$)$. The median follow-up period was 74 months. The 3 year DFS was $92.2 \%$ and $95.5 \%$ (p value 0.504 ) for AC and SCC, respectively, and the 3 year OS was $97 \%$ and $100 \%$ (p value 0.194 ).

Conclusions: Patients with adenocarcinoma tend to have a larger tumor and are more likely to receive adjuvant therapy. The histological subtype does not have an impact on survival in patients with early cervical cancer undergoing $\mathrm{RH}$.

\section{Abstract \#187: Cytological and Colposcopical Evaluation of Symptomatic Women in a Tertiary Care Center-A Study of 200 Cases}

Sonam Singh, Ranjana Desai

Umaid hospital, SN Medical College Jodhpur, Rajasthan, India

Objectives: Due to local myths or poor health services millions of women worldwide never undergo cervical cancer survey and hundreds of thousands die prematurely without ever knowing why they were ill. Cervical cancer in India is second most frequent cancer among women, burden is higher due to lack of screening and health services. Study was included to evaluate if parallel testing with PAP and Colposcopy can maximize the sensitivity for screening of CIN and Cancer Cervix. Aim of this was to screen symptomatic women irrespective of the development of premalignant and malignant lesion of cervix.

Methods: This study was conducted in 200 symptomatic women meeting with inclusion criteria, attending OPD of Obstetrics and Gynaecology department attached to Dr S N Medical College,
Jodhpur. After taking consent and history women were subjected to PAP and Colposcopy followed by Biopsy for selected cases.

Results: Out of 200 symptomatic women commonest complaint was white discharge. 3\% Women had inflammatory findings on Pap smear, $4 \%$ had ASCUS, 0.5\% ASC-H 3\% HSCL, 0.5\% HSLL, 21\% Normal Colposcopy, 38.5\% Abnormal Colposcopy, $28.5 \%$ miscellaneous finding and $12 \%$ had Indecisive.

Conclusions: Cervical cancer is a premalignant disease and has a long pre-invasive phase. With the help of screening methods like pap smear and colposcopy, cervical lesions can be diagnosed early before reaching in invasive stage in a single visit approach.

\section{Abstract \#192: A Diagnostic and Correlative Study By Colposcopy and Cytology in the Age Group of 35-45 Years}

Ananya Kaushik, Ranjana Desai

S N Medical College and Umaid Hospital Jodhpur, Rajasthan, India

Introduction: In India, carcinoma of the uterine cervix is the most common form of cancer in females. With the help of screening methods, its incidence can be reduced by identifying it in the premalignant stage. But due to ignorance at various levels and importantly women herself, it becomes difficult to achieve the target of eliminating it. For the same reason WHO recommended "once in a life time screening at the age of 40 years".

Objective: This study aimed at evaluating cervical lesions in women of the target age group, by both cytology and colposcopy and to study the correlation between the two.

Methods: This study was conducted over 500 women in the department of obstetrics and gynaecology SNMC, Jodhpur targeting the age group of 35-45 years.

Results: $54 \%$ of pap smear were normal, $42 \%$ Inflammatory, 1.2\%ASCUS, $1.8 \%$ ASC-H and LSIL, respectively, $0.8 \%$ HSIL. $38 \%$ of colposcopy findings were normal, $24 \%$ abnormal and $34.8 \%$ miscellaneous. Colposcopy-directed biopsy was taken in 85 women out of which 8 showed cervical dysplasia, 1 had CIN1, 5 had CIN II and 1 non-keratinizing squamous cell carcinoma. In women with cervical dysplasia, 75\% were among 35-40 years.

Conclusions: All the women in the age group between 35-45 years should have at least cervical screening as it not only increases the detection rate but also helps in diagnosis of other cervical pathologies. We found stronger correlation between colposcopic findings as compared to correlation between pap smear and biopsy findings. Colposcopy-directed biopsies should be the gold standard as it not only identifies cervical dysplasia but also helps in taking targeted biopsies.

\section{Abstract \#197: Comparative Study Between the Simplified Reid'S Colposcopic Index Method and the Swede Score in Early Detection of Cervical Intraepithelial Neoplasia}

Shalini Srivastava, Kavita N. Singh, Radhika Nandwani

Department of Obstetrics and Gynaecology, NSCB, Medical College and Hospital, Jabalpur, Madhya Pradesh, India

Objectives: In the present study, we compared the Simplified RCI with Swede score and correlated the findings with the histopathology reports.

Methods: In the present prospective study, 6390 women aged 25 to 65 years were screened by pap and VIA. 5181 pap smears were submitted and reported as 4049 NILM, 68 ASCUS. In Simplified reid's colposcopic index (Simplified RCI), cervix was divided into four quadrants, and examination was done in a clockwise direction 
starting from the right upper quadrant; each quadrant was scored and documented in an equation style. The colposcopic findings of 147 women were scored by Simplified RCI and Swede score and compared. Reports and findings of Pap, VIA, Simplified RCI and Swede score were correlated with histopathology reports. Diagnostic performance of pap smear, VIA, Simplified RCI and Swede score was analyzed using sensitivity, specificity, positive and negative predictive values, accuracy analysis methods. ROC analysis was also performed to calculate the area under curve (AUC) and sensitivity and specificity at various cutoff values. Pearson's correlation coefficient was calculated to test the association of Simplified RCI and Swede score.

Results: In present study for pap smear, sensitivity $75.60 \%$, specificity $100 \%$, PPV $100 \%$, and NPV $76.70 \%$ with accuracy of $86.49 \%$ were calculated. For VIA sensitivity $97.60 \%$, specificity $90.90 \%$, PPV $93 \%$, and NPV $96.80 \%$ with accuracy of $94.59 \%$ were calculated. ROC analysis showed that Pap and VIA could predict pre-invasive cervical lesions for cervical cancer at $88 \%$ and $94 \%$, respectively. Simplified RCI and Swede score had a good correlation with the correlation coefficient ${ }^{\circledR} 0.983 ; \mathrm{P}<0.0001$. AUC of ROC of both tests showed excellent performance with 0.937 for Simplified RCI and 0.936 for Swede score. At cut-off of 3 for Simplified RCI sensitivity $100 \%$, specificity $69.70 \%$, PPV $80.40 \%$, and NPV $100 \%$ with accuracy of $86.49 \%$ were calculated. At cut-off of 4 for Swede score sensitivity $97.60 \%$, specificity $60.60 \%$, PPV $75.50 \%$, and NPV $95.20 \%$ with accuracy of $81.08 \%$ were calculated.

Conclusions: Pap smear and VIA are very good and effective method for cervical cancer screening. Pap smear is less sensitive (76\%) as compared to VIA (98\%) but more specific $(100 \%)$ than VIA $(91 \%)$. Simplified RCI performed well in terms of both sensitivity (100\%) and specificity $(70 \%)$, though Swede score is also very much comparable and acceptable with sensitivity (98\%) and specificity (61\%). Simplified RCI was easier to document and to recall the colposcopic findings of particular case with formula, which makes follow-up easier. In Swede score, the criterion of size of lesion is measured in millimeters, which is difficult in clinical practice and is extrapolated as number of quadrants. In contrast to this Simplified RCI scoring system is based on lesion findings of each quadrant separately, thus making its use more user friendly.

\section{Abstract \#202: Comparison and correlation of colposcopy scores with histopathology in screening positive patients}

Aswathy G Nath, Rema P, Remani Wesley, Suchetha S, Sivaranjith J, Dhanya Dinesh

Regional Cancer Centre, Thiruvananthapuram

Background: Colposcopy was introduced to aid cervical biopsy to detect premalignant lesions of cervix. Reid, Swede and IFCPC scores were later introduced to avoid subjective variation.

Objectives: To study correlation between colposcopy scores like Reid score, Swede score and IFCPC scores with histopathology in screening positive patients.

Methods: Prospective study of patients who underwent colposcopy for cervical cancer screening from June 2018 to January 2021. Reid score, Swede score and IFCPC score were documented in colposcopy charts. Colposcopy scorings and histopathology were statistically assessed using spearman's rank correlation test.

Results: Out of total 220 females screened average age was 49.75 . Reid score $>4$ correlated with $62.3 \%$ of high-grade histopathology $(p=0.149)$, Swede score $>5$ correlated with $61.3 \%$ of high-grade histopathology report $(\mathrm{p}=0.038)$, while IFCPC score as major lesion correlated with $78.3 \%$ of high-grade histopathology $(\mathrm{p}=0.001)$. Reid score has sensitivity $62.3 \%$, specificity $47.4 \%$; Swede score has sensitivity $61.3 \%$, specificity $52.6 \%$ with histopathology. IFCPC scoring had sensitivity $78.3 \%$, specificity $47.4 \%$ with histopathology. Measurement of agreement kappa was 0.096 for Reid score, 0.139 for Swede score and 0.254 for IFCPC score. P value was significant with IFCPC score, 0.001 .

Conclusions: IFCPC scoring has a better correlation and more sensitivity in detecting high-grade lesion while swede score is more specific in detecting high-grade lesion.

\section{Abstract \#203: Correlation Between Colposcopy Directed Biopsy And LEEP In Cervical Intraepithelial Neoplasia}

Monika Vivekanand Singh, Rujuta fuke, M.M. Kamal

Department of Obstetrics and Gynecology, Department of Pathology, GMCH Nagpur, India

Objectives: To correlate between colposcopy-directed punch biopsy and loop electrosurgical excision procedure (LEEP) in cervical intraepithelial neoplasia and also to determine the prevalence of cervical intraepithelial neoplasia in patients based on abnormal colposcopy findings.

Design: Prospective Observational study.

Methods: All women attending Gynaecological Outpatient department at tertiary care centre, who had given consent for study and met the inclusion and exclusion criteria of reproductive age group (22-55 years) screened for cervical intraepithelial neoplasia (CIN) by visual inspection via acetic acid (VIA) and/or cervical cytology by liquid-based cytology. All the patients with positive VIA and abnormal cervical cytology findings will be subjected /screened by colposcopy examination. A sample size of 150 patients were taken for performing colposcopy.

Results: Total 150 were subjected to colposcopy-directed biopsy, only 39 subjects were reported to have CIN and rest of subjects were diagnosed as having cervicitis. LEEP was performed on these 39 subjects. The Kappa statistics were 0.4104 with agreement of $64.10 \%$ with $\mathrm{p}$ value 0.0001 and Spearman's correlation was 0.5335 with $\mathrm{p}$ value 0.0005 which was highly significant.

Conclusions: There is statistical correlation between colposcopy-directed biopsy and LEEP in patients of cervical intraepithelial neoplasia and LEEP is gold standard.

\section{Abstract \#210: Concurrent Use of Pap Test and Colposcopic Evaluation By Revised IFCPC 2011 Terminology in Diagnosis Of Premalignant Lesions Of Unhealthy Cervix}

Prabha Lal, Shilpi Nain

Department of Obstetrics and Gynaecology, Lady Hardinge Medical College, New Delhi, India

Objectives: Cervical cancer is the most common gynaecological cancer in developing countries. Screening all women at risk especially in developing countries is helpful in decreasing the incidence. The Objective of the present study was to evaluate suspicious cervix with routine Pap test and concurrent colposcopy using revised IFCPC terminology and to correlate cytology and colposcopic findings with histopathological findings.

Methods: A prospective cross-sectional study was done on 90 women fulfilling inclusion criteria. Cytology and office colposcopy were performed simultaneously and grading of the disease was done according to revised IFCPC terminology 2011. Biopsy was taken from abnormal area observed on colposcopy.

Results: Colposcopy and Pap test was done in 90 women with unhealthy. Out of 90, 10 cases were excluded from study as it was 
inadequate findings on colposcopy. In demographic profile mean age was 43.2 years, majority (95\%) were multipara and $(57.5 \%)$ belonged to low socioeconomic group. A total of 80 women had adequate colposcopic findings on general assessment \&were graded according to revised IFCPC 2011.35 out of $80(43.7 \%)$ had minor changes out of that $32(91.4 \%)$ were confirmed on histopathological analysis as CIN -1 \& rest 3 (9.4\%) were underdiagnosed on colposcopy as it was CIN-2/3 on histopathology. 14 out of $80(17.5 \%)$ had major changes on colposcopy. Out of which 7 (50\%) had CIN2/3 on histopathology. 4 out of $14(28.5 \%)$ had carcinoma cervix. Rest 3 out of $14(21.4 \%)$ had carcinoma in situ. IFCPC 2011 terminology is very effective in distinguishing between minor and major lesions; however, it is sometimes not helpful to distinguish between very early cancer $\& \mathrm{CIN}-2,3$ since most of the findings are overlapping. Sensitivity and specificity of Pap test was $38.4 \%$ and $87 \%$ while of colposcopy was $71.7 \%$ and $78.05 \%$

Conclusions: Colposcopic evaluation using IFCPC 2011 terminology is more descriptive and reproducible. Concurrent use of Pap test and colposcopy in evaluation of premalignant lesions of unhealthy cervix should be recommended as they are complementary to each other.

\section{Abstract \#238: Swede Score in Colposcopy for Screening Carcinoma Cervix}

Syamala. O, Jaya Choudhary

Sri Ramachandra Institute of Higher Education and research, Chennai, India

Objectives: To evaluate the efficacy of Swede score in colposcopy for screening carcinoma Cervix in correlation with histopathological examination.

Methods: A Prospective observational study was conducted at Department of Obstetrics and Gynaecology of Sri Ramachandra Institute of Higher Education and Research, Chennai between January 2019-March 2020. A total of 85 sexually active women aged 21-65 years who met the selection criteria were initially included in the study. Colposcopy examination using Swede score was done followed by colposcopy-directed punch biopsies when Swede Score was between 1 and $10(\mathrm{n}=76)$. Histopathological examination was compared with Colposcopy using Swede Score to measure the accuracy and validity of the test.

Results: Among 76 patients, Swede score colposcopy revealed 62 patients with score 1-4 suggestive of normal/low-grade lesion (LGL), 8 patients with score 5-6 suggestive of high-grade lesion (HGL) and 6 patients with score 7-10 suggestive of HGL/suspected invasive cancer. Swede score colposcopy was compatible with histopathology results in86.85\% of patients. Of the $16(21.06 \%)$ cases of biopsyproven cervical intraepithelial neoplasial (CIN), 12 (19.35\%) were rightly identified during colposcopy. Biopsy-proven cases of CIN 2/CIN3/invasive Cancer were rightly identified as HGLs by colposcopy using Swede score in this study with $100 \%$ sensitivity and 89.8\% specificity for HGL.

Conclusion: Colposcopy impression using Swede score with its good sensitivity and specificity in correlation with histopathological examination is found to be a good screening test for Cervical cancer especially in detecting HGL.
Abstract \#241: 5 Year Experience With Colposcopy In One Of The Screening Points At National Hospital, Abuja, North-Central Nigeria

Mahmoud AR, Mairami FZ

Department of Obstetrics and Gynaecology, National Hospital, Abuja.

Objectives: Colposcopy is a diagnostic procedure with a primary goal of identifying precancerous and cancerous lesions so that they may be treated early. To evaluate the Pap-smear results and colposcopic findings performed in the first 5 years of setting up one of the two colposcopy units of our hospital.

Methods: A study of 137 patients who were referred to the gynaecology clinic for colposcopy during the study period between January 2013 to December 2018 was carried out. Data was extracted from the records department of National Hospital, Abuja and same was analysed using simple statistical methods.

Results: Amongst the one hundred and thirty-seven patients studied, $69.3 \%$ were referred on account of abnormal pap smear $(49.6 \%$ of whom had a result of low-grade squamous intraepithelial lesion and $19.7 \%$ for high-grade lesion). $26.2 \%$ were referred on account of a suspicious cervix and $4.4 \%$ for persistent inflammatory smears. Abnormal colposcopic findings were reported in $49.6 \%$ of the study group who subsequently had colposcopically directed biopsies.

Conclusion: The combined use of Pap-smear and colposcopy in the detection of premalignant and malignant lesions of the cervix increases the success and enables early diagnosis and appropriate treatment.

\section{Abstract \#245: Study of Colposcopy and Histopathological Correlation in Cervical Lesions at Tertiary Care Centre}

Pranshi Asati, Shabana Sultan

Gandhi Medical College and Sultania Hospital, Bhopal, India

Objectives: Study of colposcopic and histopathological correlation for cervical cancer lesions at tertiary care centre.

Methods: This is a observational study done over one year of all outpatient and inpatient females of age more than 20 years presenting with white discharge, menstrual abnormality, abnormally looking cervix, VIA positive, sexually transmitted disease and abnormal pap smear. Data was collected from cases and analysed. Grouped data was expressed as frequency and percentage, whereas numerical data was expressed as mean and standard deviation. Correlation between colposcopy and histopathological diagnosis was established.

Results: Out of 89 cases of chronic cervicitis, $47.2 \%$ cases were diagnosed as inflammation whereas $22.5 \%$ were identified as CIN I on colposcopy. However, $18 \%$ chronic cervicitis cases were identified as normal on colposcopy. All the histopathological cases of polyp were diagnosed as polyp on colposcopy. Out of 22 cases of CIN I on histopathology, colposcopy revealed CIN I in $32 \%$ cases. Similarly, histopatho-colposcopic correlation for CINII and CIN III cases were noted in $41.7 \%$ and $36.4 \%$ cases, respectively. Out of 4 cases of squamous cell carcinoma, none of the cases were diagnosed as cancer on colposcopy. The observed correlation between histopathology and colposcopy was statistically significant $(p<0.01)$. Diagnostic accuracy, sensitivity, specificity, PPV and NPV was $77.2 \%, 78.6 \%$, $76.1 \%, 72.7 \%$, and $81.4 \%$, respectively.

Conclusions: Colposcopy has a good correlation with histopathology for identification of premalignant lesions and may obviate the need for repeated pap smear. Therefore combined approach (PAP's Smear, colposcopy, biopsy under colposcopic guidance is recommended. 
Abstract \#270: SEE AND TREAT Approach - after colposcopy in VIA positive women

\section{P. Pangtey ${ }^{1}$, S. Shamsunder ${ }^{1}$, S. Malik ${ }^{1}$, C. Ahluwalia ${ }^{2}$}

Departments of Obstetrics \& Gynaecology ${ }^{1}$ and Pathology ${ }^{2}$, V.M.M.C. and Safdarjung Hospital, New Delhi, India

Objective: See \& Treat at colposcopy has been described for highgrade abnormalities on the Pap smear with high-grade lesions on colposcopy. Visual Inspection with Acetic Acid (VIA) is a low cost screening test for LMICs. Colposcopy and treatment at the same sitting prevents loss to follow-up. The objective in the present study was to evaluate the efficacy of SEE AND TREAT approach by LLETZ in VIA positive women having high-grade lesions on colposcopy.

Design: Prospective Interventional Study.

Method: Women in the age group of 25-50 years and coming to the general gynaecology clinic had opportunistic screening with VIA; those who were VIA positive underwent Colposcopy and SEE and TREAT using LLETZ if the Colposcopy findings were suggestive of a high-grade lesion. Treatment was defined as acceptable if the histopathology showed CIN; overtreatment if there was no CIN.

Results: Colposcopy was performed in 688 women who were VIA positive; of them 101 had a high-grade lesion and underwent LLETZ. All of them had CIN on histopathology; 35 (34.6\%) had CIN 1, 55 (54.5\%) had CIN 2 and $11(10.9 \%)$ has CIN 3. The SEE and TREAT approach was acceptable in all and there was no overtreatment in any case. There were no major complications.

Conclusion: VIA followed by SEE AND TREAT approach at colposcopy is acceptable and can reduce loss to follow-up.

\section{Abstract \#271: Predictive Value of Swede Score for See and Treat in VIA Positive Women}

P. Pangtey ${ }^{1}$, S. Shamsunder ${ }^{1}$, S. Malik ${ }^{1}$, C. Ahluwalia ${ }^{2}$

Departments of Obstetrics \& Gynaecology ${ }^{1}$ and Pathology ${ }^{2}$, V.M.M.C. and Safdarjung Hospital, New Delhi, India

Objectives: Swede Score can objectively assess the grade of acetowhite lesions at colposcopy. Determining a cut-off Swede score predictive of high-grade CIN would help triage women to See \& Treat Approach by LLETZ.

Design: Prospective Interventional Study.

Method: Women in the age group of 25-50 years and coming to the general gynecology clinic had opportunistic screening with VIA and Colposcopy if VIA positive. Acetowhite lesions at colposcopy were scored by the Swede score; a cut-off for Swede score of $\geq 5$ was used to triage women for SEE and TREAT approach using treatment with LLETZ. Predictive value of each score $\geq 5$ was calculated by ROC curves using histopathology as the gold standard.

Results: Colposcopy was carried out for 688 women who were VIA positive; 101 had a colposcopy score of $\geq 5$ and underwent LLETZ, all had CIN on histopathology. Swede score was between 5-7 in 91 women (90.10\%), 36.26\% had CIN 1 while $63.74 \%$ had CIN 2+. The score was $\geq 8$ in $10(9.90 \%)$ of them, $20 \%$ had CIN 1 and $80 \%$ had CIN $2+$. The predictive value for CIN $2+$ was best at a score of $>5$.

Conclusion: Swede Score of 5 best identifies a high-grade lesion suitable for SEE AND TREAT approach by LLETZ.
Abstract \#277: A comparative study between the Simplified Reid's colposcopic index method and the Swede score in early detection of cervical intraepithelial neoplasia at NSCB medical college Jabalpur

Shalini Srivastava ${ }^{1}$, Kavita N. Singh ${ }^{1}$, SonalSahni ${ }^{1}$, Radhika Nandwani $^{2}$, M. P. Singh ${ }^{3}$

${ }^{\mathbf{1}}$ Department of Obstetrics and Gynaecology, ${ }^{2}$ Department Of Pathology, N.S.C.B Medical College and Hospital, Jabalpur, (M.P.) ${ }^{3}$ Statistician ICMR - NIMR FS

Objective: In the present study, we compared the simplified RCI with Swede score and correlated the findings with the histopathology reports.

Methods: In the present prospective study, 6390 women aged 25 to 65 years were screened by Pap smear and VIA. Colposcopic findings of 147 women were scored by Simplified RCI and Swede score; and compared. Reports and findings of Pap, VIA, Simplified RCI and Swede score were correlated with histopathology reports. Diagnostic performance of pap smear, VIA, Simplified RCI and Swede score was analysed using sensitivity, specificity, positive and negative predictive values, accuracy analysis methods. ROC analysis was also performed to calculate the area under curve (AUC) and sensitivity and specificity at various cutoff values. Pearson's correlation coefficient was calculated to test the association of Simplified RCI and Swede score.

Results: In present study, for pap smear sensitivity $75.60 \%$, specificity $100 \%$, PPV $100 \%$, and NPV $76.70 \%$ with accuracy of $86.49 \%$ were calculated. For VIA sensitivity $97.60 \%$, specificity $90.90 \%$, PPV $93 \%$, and NPV $96.80 \%$ with accuracy of $94.59 \%$ were calculated. ROC analysis showed that Pap and VIA could predict pre-invasive cervical lesions for cervical cancer at $88 \%$ and $94 \%$, respectively. Simplified RCI and Swede score had a good correlation with the correlation coefficient (r) $0.983 ; \mathrm{P}<0.0001$. AUC of ROC of both tests showed excellent performance with 0.937 for Simplified RCI and 0.936 for Swede score. At cut-off of 3 for Simplified RCI sensitivity $100 \%$, specificity $69.70 \%$, PPV $80.40 \%$, and NPV $100 \%$ with accuracy of $86.49 \%$ were calculated. At cut-off of 4 for Swede score sensitivity $97.60 \%$, specificity $60.60 \%$, PPV $75.50 \%$, and NPV $95.20 \%$ with accuracy of $81.08 \%$ were calculated.

Conclusions: In the present study, we found that Pap smear is less sensitive (76\%) as compared to VIA (98\%) but more specific $(100 \%)$ than VIA $(91 \%)$. Simplified RCI performed well in terms of both sensitivity (100\%) and specificity (70\%), though Swede score is also very much comparable and acceptable with sensitivity (98\%) and specificity (61\%). Simplified RCI was easier to document and to recall the colposcopic findings of particular case with formula, which makes follow-up easier. In Swede score, the criterion of size of lesion is measured in millimetres, which is difficult in clinical practice and is extrapolated as number of quadrants. In contrast to this Simplified RCI scoring system is based on lesion findings of each quadrant separately, thus making its use more user friendly.

\section{Abstract \#282: Direct colposcopy versus colposcopy in screen positive women for detection of precancerous and cancerous lesion of cervix}

Vijay Zutshi, Guneet Kaur, Sachin Kolte

VMMC\& Safdarjung hospital, Delhi, India

Objective: To assess the role of direct colposcopy for screening of precancerous and cancerous lesion of cervix.

Methods: A cross-sectional study was done in the department of OBGY and department of pathology, VMMC \& SJH for 12 months. An opportunistic screening done in the OPD post which screen 
positive women either by visual inspection by acetic acid or Lugol's iodine/PAPS/HPV DNA and followed by colposcopy. Direct colposcopy was done in women having unhealthy-looking cervix. Women with positive findings on colposcopy were subjected to fourquadrant directed cervical biopsy. Pregnant women, women having active vaginal bleeding or any past treatment for cervical lesion were excluded from the study.

Results: A total of 70 colposcopies were done out of which 35 were done in screen positive women (Group 1) and 35 were direct colposcopy for unhealthy looking cervix (Group 2). Demographic data like median age, mean parity, lower socioeconomic status, age $<$ 18 years at first sexual contact, first child birth at age $<18$ years, addictions (smoking/tobacco) and contraceptive use was comparable in both groups.

Colposcopy was adequate in all women. Abnormal colposcopy was noted in $75 \%$ patients in Group 1 (55\% minor grade \& $20 \%$ major grade) and $60 \%$ patients in Group 2 (38\% minor grade \& $22 \%$ major grade). In Group 1 cervical biopsy showed benign pathology in 16 , CIN 1 in 6, CIN $2 / 3$ in 3 \& malignancy in 1 woman and in Group 2 benign in 14, CIN 1 in 3, CIN 2/3 in 3 and malignancy in 1 woman. Conclusion: Direct colposcopy is a useful method to detect precancerous and cancerous lesions of cervix.

\section{Abstract \#283: Colposcopy in unhealthy cervix: an essential tool needed without awaiting initial cytology \\ Rekha Sachan, Ayushi Shukla, PL Sankhwar, Uma Singh \\ Department of Obstetrics and Gynaecology, King George's Medical University, Lucknow, India}

Objective: Evidence for using colposcopy in absence of a squamous intraepithelial lesion on conventional cytology has an important position in cervical cancer screening. Aim of this study was to evaluate diagnostic accuracy of colposcopy against cytology in unhealthy cervix for timely detection of preinvasive and invasive cervical lesions.

Method: Prospective study. 496 diagnostic colposcopies on women with unhealthy cervix on examination were performed in one year. Concurrently cytology was done as routine protocol. 99 women having high-grade colposcopy (Swede score $\geq 5$ ) underwent guided biopsy and tissue was evaluated histopathologically. Cytology of same patients were compared.

Results: Mean age of women was $42.68 \pm 11.69$ years and mean age at consummation of marriage $20.94 \pm 3.21$ years. Cervical erosions were common findings $(41.4 \%)$ followed by hypertrophied cervix $(33.3 \%)$ and cervix bleeding on touch (17.2\%). $24.14 \%$ women with ASCUS cytology had high-grade histopathology (CIN2 +), while 42.3\% women with LSIL had high-grade histopathology (CIN2 +). Taking ASCUS and above as cut-off, the sensitivity, specificity of Pap smear was $97.8 \%, 14.6 \%$ and diagnostic accuracy was $55.3 \%$. For overall analysis of colposcopy Swede Score at $\geq 5$, colposcopy was $100 \%$ sensitive and $41.2 \%$ specific and diagnostic accuracy was $69.7 \%$. At swede score $\geq 7$, colposcopy was $64.6 \%$ sensitive and $100 \%$ specific and diagnostic accuracy was $82.8 \%$.

Conclusion: Women with cytology reports of ASCUS and LSIL had CIN $2+$ on histopathology thereby highlighting drawbacks of conventional cytology. Colposcopy can be used as a valuable concurrent tool in women with unhealthy cervix and high-grade colposcopy can be taken up for biopsy without awaiting initial cytology in such women.
Abstract \#313: Correlation between Colposcopic Impression using Reid's Colposcopy Index and Histopathological Grading of Premalignant Lesions of Cervix

Raksha. S, Vijay Nidhi Dhasmana

Department of Obstetrics and gynaecology Deenanath Mangeshkar Hospital and Research Centre Erandwane, Pune, Maharashtra, India

Background and Objective: The Reid's Colposcopy Index (RC) relies on critical analysis rather than on pattern recall. The primary objective was to assess the agreement between colposcopy and histopathological grading of lesions of cervix. The secondary objectives were to correlate social demographic data with incidence of cervical preinvasive and invasive lesions and to determine the diagnostic efficacy of colposcopy.

Methods: One hundred sexually active women above 18 years age with complaints of vaginal discharge, intermenstrual or postcoital bleeding, unhealthy-looking cervices, nonconclusive/abnormal cytology report were included in a single center, prospective, observational study over 2 years. Screen positive women using Pap smear were reffered for colposcopy. Reid's Colposcopy Index was calculated and biopsy was taken at site with the highest score and sent for histopathology (HPE). Colposcopy diagnosis according to RC were compared with HPE and sensitivity, specificity, positive predictive value (PPV), negative predictive value (NPV), accuracy and strength of correlation was observed. Statistical significance was derived using weighted Kappa " $\mathrm{k}$ " correlation coefficient and $\mathrm{p}$ value.

Results: Colposcopy when compared to histopathology had a sensitivity of $100 \%$, specificity of $48.3 \%$, with PPV of $22.4 \%$ and NPV of $100 \%$. The $\mathrm{p}$ value was 0.001 suggesting a significant strength of agreement between colposcopy diagnosis using Reid's index and histopathological diagnosis.

Conclusion: Using a scoring system like RCI can help in reducing interobserver variability and may be useful for further follow-up of low-grade lesions, provided site specification is added. Hence, it greatly simplifies learning colposcopy.

\section{Abstract \#320: Newer Colposcopic Signs-How Accurate Are They?}

R. Induja, Abha Singh, Prabha Lal

Department of Obstetrics and Gynaecology, Lady Hardinge Medical College, Delhi

Objective: To look for the presence of colposcopic signs inner border, ridge and rag sign in women with abnormal cervical cytology and to correlate the colposcopic findings with histopathology.

Design: Cross-sectional observational study.

Methods: Eighty-two women with abnormal cervical cytology were included. A detailed history was taken and examination done followed by colposcopic assessment of the cervix, with special attention to inner border, ridge and rag signs. Colposcopic-guided biopsies were obtained. Taking histopathology as gold standard the diagnostic accuracy of colposcopic signs was calculated.

Results: Grade 2/3 aceto-white areas were present in 38 cases. Inner border sign was seen in 9 cases, ridge sign in 21 cases and rag sign in 13 cases. The sensitivity, specificity, positive Predictive Value, Negative Predictive Value, diagnostic accuracy of Inner border sign was $28.12 \%, 100 \%, 100 \%, 68.49 \% \& 71.95 \%$, respectively; for Ridge sign was $65.62 \%, 100 \%, 100 \%, 81.97 \% \& 86.59 \%$, respectively; for Rag sign was $37.5 \%, 98 \%, 92.31 \%, 71.01 \%$ \& $74.39 \%$, respectively. Conclusions: The presence of these colposcopic signs is highly predictive for high-grade cervical intraepithelial neoplasia. These signs are easy to learn, less time consuming and have better 
diagnostic accuracy. While cytology remains the gold standard for screening of carcinoma cervix, it is important to complement it with colposcopic pathognomic signs-guided biopsy for early detection and management of cervical carcinoma.

\section{Abstract \#323: Predictive value of VIA, HPV genotyping and Colposcopy in opportunistic cervical screening}

Harshitha K, Saritha Shamsunder, Sunita Malik, Vijay Zutshi, Rashmi Arora, Anoop Kumar, Mohini Agarwal

Department of Obstetrics and Gynaecology, VMMC and Safdarjung Hospital, New Delhi, India

Objective: To find the predictive value of VIA, HPV genotyping and colposcopy for predicting CIN in women screened by opportunistic primary HPV screening.

Design: Cross-sectional observational study.

Method: Testing for high-risk human papillomavirus by conventional PCR was carried out in 1606 sexually active women $\geq 30$ years attending the gynaecology clinic, 137 women were HPV positive who had HPV genotyping, Visual Inspection with Acetic acid (VIA), colposcopy and biopsy sequentially. The sensitivity, specificity, PPV \& NPV at $95 \%$ CI of VIA positivity and AUC for predicting CIN $2+$ was compared using histopathology as the gold standard.

Results: The HPV prevalence rate in the 1606 women screened was $8.5 \%$. The sensitivity of HPV 16,18 genotyping was $82.1 \%$ (95\% CI $63.11 \%$ to $93.94 \%)$ for VIA $71.43 \%(51.33 \%$ to $86.78 \%)$ and Colposcopy was $96.43 \%$ ( $81.65 \%$ to $99.91 \%)$. The specificity was $13.04 \%$ (95\% CI $6.93 \%$ to $21.68 \%$ ) for HPV 16,$18 ; 43.48 \%$ (33.17\% to $54.22 \%$ ) for VIA and $30.43 \%$ (21.27\% to $40.90 \%)$ for Colposcopy. ROC area under curve for HPV 16,18 was 0.48 (95\% CI 0.38 to 0.57 ), VIA was 0.57 (95\% CI 0.48 to 0.66 ) and Colposcopy was 0.63 (95\% 0.54 to 0.72 ). Colposcopy missed one case of CIN 2+, HPV 16,18 missed 5 and VIA missed 8.

Conclusion: Direct colposcopy should be considered in women detected screen positive at the health facility to minimize loss to follow-up and detect and effectively treat high-grade CIN.

\section{Cervical Cytology}

Abstract \#28: Cervical cytologies suspected of infiltrative injury from the screening program in asymptomatic patients

Rafael JoseNavarro Avila, Rubio Lorenzo, Rocio Bermejo, Ana Belen Bolivar de Miguel, Reyes Oliver Perez, Alvaro Diez

Hospital Universitario 12 de Octubre, Spain

Objectives: The aim of this study is to analyze the cases assessed in our center between 2013 and 2018 with cervical cytologies suspected of infiltrative injury from the screening program in asymptomatic patients in force in our area and the cytohistological correlation after its study in the cervical pathology unit.

Methods: Retrospective descriptive analysis of a total of 10 patients who, after performing the current population oppotunistic screening, were referred to our cervical pathology unit with the result of cervical cytology suspected of infiltrative injury between 2013 and 2018. The subsequent statistical analysis was performed with SPSS 25.

Results: All cytologies of our study (10) were reported as suspected of malignancy. The result of the subsequent biopsy showed 50\% CIN $3,10 \% \operatorname{CIN} 2,10 \%$ in situ adenocarcinoma, $10 \%$ carcinoma, in one case no biopsy was performed and in another case the biopsy was negative.
The subsequent pathological results after conization were the following: two cases of squamous cell carcinoma (20\%), one case of adenocarcinoma $(10 \%)$, and the rest were informed as no cancer (70\%). Therefore, of the 10 cytologies that were initially reported as suspected of malignancy, the diagnosis of malignant pathology was confirmed in $30 \%$ of them.

Conclusions: In our study group, cyto-histological concordance is lower for suspected infiltration than that described in the literature. However, the small sample size does not allow obtaining statistically significant comparative results. It should also be noted that these cytological findings only come from patients from population screening without prior clinical suspicion.

\section{Abstract \#98: Comparison of the Pap smear and PCR test} for the detection of HPV in Cervical specimens of Indian Women

Ganesh Kumar S, Kurupakkar P, Chirayu P, Giridharan A

*LifeCell International Pvt. Ltd, Chennai, *Sathyabama Institute of Science and Technology, Chennai, India

Objectives: Cervical cancer is, at present, the second most frequent cancer in women in the world. Global incidence of 470,000 new cases per year. To investigate the presence of HPV in the Cervical region and determine predominant serotype expression of HPV by various techniques.

Methods: This study was performed on 334 patients in the age groups ranging from 21 to 85 years. Liquid-Based cytology samples were collected from each woman and cytological test was performed using Pap smear; HPV DNA identification and serotyping were performed using PCR method and Genome sequence.

Results: Out of 334 samples, 18 samples were screened positive for HPV by PCR technique and showed negative for intraepithelial lesion or malignancy cytological features on Pap smear study. The overall HPV DNA positivity is $5.4 \%$ by PCR compared to $0 \%$ by Pap smear. Overall study shows that, LR serotype-6 was identified in $17 \%$ compared to HR serotype-16 which was identified in $6 \%$ of HPVpositive cases.

Conclusions: PCR-HPV DNA detection tests may be ideal in clinical settings is to accurately detect all HPV infections that even women with normal cytology. Compared to HR serotype 16, LR serotype-6 is detected more in PCR-based assay. Continuous follow-up for the LR HPV cases to confirm the change in risk factor.

\section{Abstract \#103: Correlation of HPV infection with different cervical abnormalities (benign and premalignant lesion): A prospective study}

Roli Purwar, Priyadarshini

Department of Obstetrics and Gynaecology, AIIMS Gorakhpur, UP, India

Objectives: To study the association of human papillomavirus (HPV) with various types of benign and premalignant cervical abnormalities. Methods: The study was conducted in tertiary care hospital between January 2014 to December 2015. After taking due informed consent, 500 sexually active women reporting to obstetrics and gynecology outpatient department were recruited. Inclusion criteria were clinically detected cervical abnormalities or abnormal cytology or histopathology. Patients diagnosed with malignancy were excluded. Among these, 50 patients were randomly selected for HPV DNA testing with abnormal cervical cytology. Patients having positive VIA; VILI or pap smear findings underwent colposcopy-guided biopsy. 
Results: Most common cervical abnormality clinically was erosion (28\%), and cevical hypertrophy (25\%) bleeds on touch (18\%). Most common cervical abnormality on cytology was bacterial vaginosis $(32 \%)$, candidiasis and trichomonas (15\%), squamous metaplasia (12\%). Most common cervical abnormality on colposcopy-guided cervical biopsy was chronic cervicitis (65\%), mild dysplasia (5\%), severe dysplasia in $3 \%$. Among cytologically positive patients $12 \%$ had positive HPV DNA. HPV 11 was positive in LSIL lesions and HPV 16 was positive in HSIL lesion. one patient with CIS positive on cytology had HPV $11+$ and also one patient with inflammatory smear had HPV11 + . On correlating cervical cytology with HPV sampling, p value was 0.004 and RR was 1.4 which shows good correlation.

Conclusions: HPV positivity was significantly high in benign and premalignant lesion of cervix, so all women with cervical abnormality should be screened for HPV. All inflammatory smears should also be kept in follow-up.

\section{Abstract \#122: Correlation of Pao smear with histopathological diagnosis in cervical cancer screening}

Chinmoyee Kalita, Shruti Bhatia

Department of Gynae Oncology, Narayana Super speciality Hospital, India

Objectives: To correlate Pap smear findings with histopathology reports and to assess the efficacy of Pap smear in cervical cancer screening.

Methods: It was a prospective study conducted in Department of Gynaecologic Oncology at Action Cancer Hospital from March 2018 to February 2019. Total 59 symptomatic sexually active women who had undergone both Pap smear and colposcopy-directed cervical biopsy were included in this study. Correlation between cytology and histopathology was analysed. Qualitative variables were correlated using Chi-Square test/Fisher's exact test. Diagnostic test was used to calculate sensitivity, specificity, negative predictive value (NPV), positive predictive value (PPV). Inter-rater kappa agreement was used to find out strength of study.

Results: Out of 59 Pap smears, maximum patients (35.59\%) belonged to age group $41-50$ years. Mean age was 43.32 years. On Pap smear, $54.24 \%$ had NILM, $15.25 \%$ had LSIL, $15.25 \%$ had HSIL, $6.78 \%$ had ASCUS,5.08\% had ASC-H and 3.39\% had SCC. $74.58 \%$ had HPV testing, out of which $59.09 \%$ were positive for high risk HPV DNA. On histopathology, $40.68 \%$ had chronic cervicitis (CC), $18.64 \%$ had CC with squamous metaplasia, $15.25 \%$ had CIN $1,10.17 \%$ had CIN 2, $6.78 \%$ had CIN 3 and $8.47 \%$ had SCC. Overall sensitivity, specificity, PPV, NPV, and strength of study were $83.33 \%, 80 \%, 74.07 \%, 87.5 \%$ and 0.621 (Kappa value). Out of 59 cases, 48 cases $(81.36 \%)$ diagnosed on Pap smear correlated on histopathology.

Conclusions: Maximum number of Pap smear findings correlated on histopathology. So Pap smear can be used as a good screening tool for cervical cancer screening.

\section{Abstract \#136: Prevalence of abnormal Pap smear by liquid- based cytology in antenatal women}

Ankita Sinha, Mukul Singh

Department of Obstetrics and Gynaecology, VMMC and Safdarjung Hospital, New Delhi, India

Objectives: This study aimed to know the prevalence of abnormal Pap smear in pregnant women by liquid-based cytology.
Methods: Cross-sectional study done at a tertiary care hospital, India from August 2017 to January 2019. Six hundred pregnant women attending the OPD at their first visit were enrolled and after taking history and clinical examination, subjected to cervical cancer screening by liquid-based cytology (LBC).

Results: Out of 600 samples, 10 samples $(1.67 \%)$ were unsatisfactory for evaluation due to low cellularity. Among 590 samples, only 2 reported as LSIL (Low-grade squamous intraepithelial lesion) and 588 reported as NILM (Negative for intraepithelial lesion or malignancy). Inflammation of varying degree, mild, moderate and dense was reported in $58.65 \%, 24.92 \%$ and $16.44 \%$, respectively. Infection was reported in $3.73 \%$ and Candida, Trichomonas and Gardnerella species were present in $2.71 \%, 0.51 \%, 0.51 \%$, respectively. Statistically significant correlation ( $\mathrm{p}$ value $<0.0001$ ) was seen between level of education and knowledge about cervical cancer screening. A statistically significant correlation ( $p$ value 0.013 ) was also seen between abnormal per speculum cervical findings and prevalence of infection in the LBC report of the screened antenatal women.

Conclusion: Antenatal period can be used as an opportunity to screen women for cancer cervix. There is low number of unsatisfactory smears with liquid-based cytology, only $1.67 \%$ of the smears were unsatisfactory for evaluation. Added advantage of doing cervical cytology in pregnancy is that in some women infective organisms can be picked up.

\section{Abstract \#141: Local experience of dual staining to categorize ASC-US, ASC-H, AGC, L-SIL cytology results}

SilvioTatti, Laura Fleider, Florencia Falcon, Maldonado Veronica, Lilí Díaz

Hospital de Clínicas “José de San Martín”, Argentina

Objectives: To analyze the performance of dual staining (DS) to categorize ASC-US, ASC-H, AGC, L-SIL PAP Smears in women 18-30 years.

Methods: After obtaining an informed consent and a complete medical record; exo and endocervical samples were taken and fixed on two slides, split sample technique (one sample for conventional cytology and other for DS). Also COBAS $4800^{\mathrm{TM}}$ was performed to establish relationship with HPV. A colposcopy was performed on all patients and a cervical biopsy was done if needed as gold standard. Sensitivity, specificity and negative predictive value were performed to triage $\mathrm{CIN} 2+$.

Results: DS was performed on 155 patients of the study group: ASCUS: 64/155, LSIL 80/155, ASC-H 9/155, AGC 2/155.

- ASC-US: negative 47/64 (73.44\%), 22/47 L-SIL histologically confirmed; positive 13/64 (20.31\%), 4/13 HSIL and 4/13 LSIL histologically confirmed. $4 / 64$ cases were not evaluable.

- L-SIL: negative 49/80 (61.25\%), 1/49 HSIL and 27/49 LSIL histologically confirmed, positive 28/80 (35\%), 1/28 AIS, 5/28 HSIL and 19/28 LSIL confirmed by biopsy $3 / 80$ were not evaluable.

- ASC-H: negative 2/9 (22.22\%), no colposcopic lesion, positive 7/9 (77.78\%), 4/7 HSIL, 2/7 LSIL histologically confirmed.

- AGC: negative 1/2 (50\%), positive $1 / 2(50 \%)$. Both with no colposcopic lesion.

Sensibility of DS for HSIL was $96.43 \%$ (95\% CI $87.77-100$ ), specificity $83.98 \%$ (95\% CI 79.04-88.93), NPV 99, 49\% (95\% CI 98.23-100). Patients with positive DS have RR 2.76 (95\% CI 1.51-5.04) for having an infection with HPV16 ( $\mathrm{p}=0.0007)$.

Conclusions: The use of DS in low-risk cytology increases the detection of patients with high-grade. 


\section{Abstract \#185: Cervical Pap Smear Pattern-Comparison Of} Hospital And Screening Camp Population

Neeraj B, Sunitha Balakrishnan, Sankar S

Department of Pathology, Government Medical College Kottayam, Kerala, India

Objectives: Cervical cancer is one of the leading cause of mortality and morbidity among women worldwide. In India, it accounts for $25 \%$ of cancer deaths. Only limited number of studies are available on prevalence of cervical epithelial abnormalities, especially in Kerala. The objective of the study was to estimate the prevalence of cervical cytological pattern in central Kerala, so appropriate screening strategies can be planned.

Methods: A 1-year descriptive study was conducted on Cervical pap smears in study population groups: (1) Camp population from various camps conducted in and around Kottayam and (2) Hospital population and received in the department of Pathology, Government Medical College, Kottayam during the study period of 12 months. Smears were assessed according to the revised Bethesda System 2014. A total of 240 cases of Pap smears were analysed in each group of population (hospital and screening camp).

Results: The mean age of population in the present study was $48.20 \pm 10.224 \mathrm{SD}$ in camp and $48.23 \pm 11.339 \mathrm{SD}$ in hospital population. Majority belongs to the age group $41-50$ years. In both majority of the smears were negative for intraepithelial lesion or malignancy (NIL/M). Epithelial abnormality was seen in $1.25 \%$ cases (LSIL) of camp population \& $2.5 \%$ cases (ASC-US, LSIL and HSIL $0.80 \%$ each) of hospital population.

Conclusion: Our study shows a relatively low prevalence of cervical epithelial abnormality similar to developed countries. Pap smear is an effective screening procedure to detect cervical cytological abnormalities. Routine screening should be offered to all women above 21 years. It is recommended to improve awareness about disease among females.

\section{Abstract \#221: Pap Smear, VIA \& HPV-DNA Testing in Screening of Premalignant Lesions of Cervix \\ Dibakar Debbarama, Salil Bindu Chakraborty \\ Agartala Government Medical College, India}

Objectives: To evaluate feasibility of screening tools VIA\& HPVDNA in detection of premalignant lesions of cervix. To evaluate simultaneous testing feasibility of screening tools of VIA and HPVDNA in detection of premalignant lesions of cervix taking Pap smear as gold standard.

Design: Cross-Sectional Study.

Methods: Study was done in Department of Obstetrics and Gynaecology OPD, AGMC\& GBPH from JANUARY 2018 to JUNE 2019, total 360 patients aged (30-65) were subjected for study and simultaneous test was done.

Results: PAP positive $17.5 \%$, PAP negative $82.5 \%$. Positive case includes LSIL $6.11 \%$, HSIL 6.94\%, malignancy $3.88 \%$. HPV-DNA positive $24.16 \%$ and negative cases are $75.83 \%$. VIA positive $20 \%$ and $80 \%$ were negative. VIA compared to final diagnosis was $57.14 \%$ with specificity of $89.89 \%$, PPV of $48.27 \%$ and NPV of $77.16 \%$. HPV-DNA compared to final diagnosis was $83.66 \%$ with specificity of $89.22 \%$, PPV of $56.16 \%$ and NPV of $76.58 \%$. The net sensitivity of both HPV DNA and VIA compared to final diagnosis $95 \%$, specificity $80.1 \%$, PPV $50.42 \%$, NPV $98.75 \%$.

Conclusions: The role of pap smear for cervical cancer screening has long been established in the West but it is not a very feasible test for a resource-limited setting. Alternative methods such as VIA may be very useful in a resource-limited setting but VIA has low sensitivity and may not perform well as stand-alone tests, so adjunctive tests are one way of improving specificity of the test. Our study has confirmed simultaneous assessment of VIA \& HPV-DNA gives an excellent positive predictive value in cervical screening.

\section{Abstract \#255: Analysis of borderline nuclear changes in squamous cells on triage cervical smear with primary HPV screening}

Manju Sree, Dr. Aye Aye, Mr. V. Wong

Princess Royal Hospital, University Hospitals of Sussex NHS Trust, UK

Objective: Analyse the outcome of borderline nuclear changes in squamous cells on triage cytology and primary HPV screening.

Design: Retrospective data collection over a period of 2 years.

Method: All patients referred to colposcopy clinic in a University Teaching Hospital with borderline nuclear changes in squamous cells on cytology triage after primary HPV screening formed the cohort. The colposcopy impression, biopsy, histology and follow-up were analysed.

Results: 486 patients were identified and followed through.

Conclusions: $6 \%$ of the study population were identified to have high-grade disease and $12 \%$ had low-grade disease. Hence referrals for borderline cervical smears need to be dealt with vigilance.

\section{Abstract \#290: Prevalence of abnormal cervical cytology in women with STIs}

Versha Dhama ${ }^{1}$, Saritha Shamsunder ${ }^{1}$, Dr Niti Khunger ${ }^{2}$, Dr Sachin Kolte $^{3}$

${ }^{1}$ Department of Obstetrics \& Gynaecology, ${ }^{2}$ Department of Dermatology \& Venereal Diseases, ${ }^{3}$ Department of Pathology, VMMC \& Safdarjung hospital, Delhi, India

Objective: To know the prevalence of abnormal cytology in women with symptoms of STI (Sexually Transmitted Infections).

Design: Cross-sectional study at a teaching hospital in Northern India.

Methods: All Women attending STI clinic and gynaecology clinic with suspicion of STI, had a detailed history and examination, speculum examination and pap smear taken by LBC using the SurePath sampler. Blood samples for HIV \& VDRL were taken from all; women with vaginal discharge had swabs from vagina, urethra \& cervix for wet mount, gram \& Giemsa stain, microscopy and culture for trichomonas, candida, gonorrhea, chlamydia, bacterial vaginosis, Haemophilus ducreyi and $\mathrm{KOH}$ mount for candida.

Results: There were 222 women in the age group of 21-60yrs (mean age $28 \mathrm{yrs})$. The STIs detected were candida in 58 (26.1\%), bacterial vaginosis in $12(5.4 \%)$, syphilis in $13(5.8 \%)$, herpes genitalis in 10 $(4.5 \%)$, genital warts in $32(14.4 \%)$, HIV in $8(3.6 \%)$, molluscum contagiosum in $34(15 \%)$, trichomonas vaginalis in $2(0.9 \%)$, ureaplasma/mycoplasma in $2(0.9 \%)$. No specific organism were found in $47(21.1 \%)$. Overall $7.6 \%$ of these women showed abnormal cervical cytology, inflammatory in $8(3.6 \%)$, ASCUS in $4(1.8 \%)$, LSIL in 2 $(0.9 \%)$.

Conclusion: Women with STI have high risk of developing cervical cancer and should be screened and followed up regularly. 


\section{Abstract \#325: Conventional Pap Vs Low-Cost Liquid-Based Cytology Technique (EziPREP)}

Meesha Zaheer, Rupali Bavikar

Dr D.Y.Patil Medical College and Hospital, Pimpri, Pune, Maharashtra, India

Objectives: To compare cytomorphological features and cytologic diagnosis on Conventional Papanicolaou (CP) smears with liquidbased cytology technique, EziPREP ${ }^{\mathrm{TM}}$, for cervical smears

Design: A prospective study with consecutive cervical sampling was conducted on 100 women attending the OPD at our institute.

Methods: CP smears were prepared as per the standard technique using Ayre's spatula. EziPrep ${ }^{\mathrm{TM}}$ required sample collection with endocervical brush followed by detaching the head of brush into the fixative vial. The EziPREP ${ }^{\mathrm{TM}}$ samples were then processed as per the manufacturer's protocol. Both CP and EziPREP ${ }^{\mathrm{TM}}$ smears were stained using standard Papanicolaou as well as H\&E staining protocols. Slides were analysed by two pathologists independently.

Results: The unsatisfactory rate for $\mathrm{CP}$ was $16 \%$, main cause being obscuring haemorrhage and inflammation, whereas on EziPrep ${ }^{\mathrm{TM}}$ samples it was $4 \%$, owing to inadequate cellular material. Staining quality was better in EziPREP ${ }^{\mathrm{TM}}$. The morphological details were comparable in both sets of smears. The pathogenic organisms, like Candida and Trichomonas, were better appreciated in EziPREP ${ }^{\mathrm{TM}}$ samples. Cellular atypia was better appreciated in CP smears. There was a $98 \%$ concordance in cytologic diagnosis between $\mathrm{CP}$ and EziPREP $^{\mathrm{TM}}$ smears. Screening time was notably reduced in EziPREP $^{\mathrm{TM}}$ smears $(2 \pm 0.5$ minutes vs $4 \pm 0.5$ minutes for $\mathrm{CP})$.

Conclusion: Owing to the better smear quality, reduced number of unsatisfactory smear and reduced screening time, EziPREP can be considered superior to Conventional Pap smear. The availability of such comparatively low-cost devices may help in wider application of liquid-based cytology cervical screening in low-resource countries.

\section{Screening in low-resource setting}

Abstract \#29: Assessment of Knowledge and Attitude of Cervical Cancer Screening among the Bangladeshi Women-A Study in a Tertiary Hospital

Afzalunnessa Chowdhury, Taslima Begum, Moumita Roy

Labaid Specialized Hospital, Bangladesh

Objectives: Cervical cancer is the second-most prevalent cancer of women in Bangladesh. It is one of the major public health problems due to high mortality rate as most patients $(80 \%)$ are diagnosed in the advanced stage. It is mostly preventable if detected early by screening test. The main purpose of the study is to assess the level of knowledge and awareness of cervical cancer prevention and screening among the women attending OPD of Gynae and Obstetrics.

Methods: This cross-sectional study was performed among the women attending the OPD of Obstetrics and Gynaecology in Shaheed Suhrawardy Medical College Hospital from June 2018-September 2018. A total of 300 respondents were selected by random sampling. All the information collected in a structured questionnaire and data were analyzed using SPSS.

Results: The majority of our respondents reported having very poor knowledge about cervical cancer. Mostly it is related with the women with low levels of general health education \& illiteracy. Only $31.23 \%$ women reported to have some knowledge about cervical cancer wanted to perform screening. Poor knowledge of husbands, his occupation, unwillingness, monthly income and age of the women observed other associated factors. Majority of respondents $(83.31 \%)$ had no idea of HPV vaccination. Women aged over 40yrs were unaware about cervical cancer.

Conclusion: Most of the women do not know clearly about cervical cancer prevention strategy, risk factors, screening and vaccination. Health education, promotion of screening program, raising awareness about its vaccination is most cost-effective approach in reducing incidence of cervical cancer in low-resource countries like Bangladesh.

\section{Abstract \#44: Nurses role in a rural cervical cancer screening program in a low-resource setting}

Narayanamma M, Pranitha J, Rukhiya Begum, Glory Hyma, Yadamma CH, Anuradha O

Institute for Rural Health Studies, Telangana, India

Objectives: Cervical cancer is both preventable and curable if detected early. In resource-constrained settings nurse's role becomes very crucial, where screening and treatment is taken close to their door steps especially in rural and remote areas. We report the role of trained nurse midwives in effective implementation of cervical screening program with remote supervision in a district hospital.

Methods: We have retrospectively analysed various activities undertaken by the trained nurses from an ongoing organized cervical cancer screening program in Mahabubnagar district, Telangana state, India from the year 2007 onwards. Nurses recruited eligible women by providing facts about prevention of cervical cancer, ensured their consent for screening, performed VIA screening, informed the test results, offered appropriate treatment and advice if found abnormal test results, encouraged non-responders to use these services in accordance with their culture, etc.

Results: Over 51,000 women between 25-60 years were motivated and screened opportunistically in the Women's cancer screening clinic of district hospital during 2007-2019 (12 years). Nearly 300 women with invasive cancers were identified and about the same number of women with pre-cancers were detected and treated. A vast majority of genital infections were also treated.

Conclusion: Intensive training, timely guidance and supervision to the nurses is an ideal solution where public health resources are limited. These nurses are a perfect alternative to a physician in cervical screening and treatment. Their routine work can be easily supervised remotely and guided through if complication arises.

\section{Abstract \#73: Correlation of Pap smear and colposcopy in relation to histopathological findings in detection of cervical precancerous lesions in rural setup}

\section{NavyaThokamooru}

RDT hospital, Bathalapalli, Ananthpur, India

Objectives: Correlation of papanicolaou (PAP) smear and colposcopy in the detection of premalignant lesions of cervix.

Methods: Women aged 20-65 years attending Gynecology OPD. Women with frank lesions, acute PID, and those treated for carcinoma cervix.

Methods: For the study group Pap smear was taken after taking informed consent. Colposcopy was done for women with positive Pap smear (LSIL, HSIL, ASCUS \& AGUS) with informed consent and explanation of the procedure. Colposcopy-directed biopsy. Histopathology reports correlated.

Results: Among total study Pap smears, only $4 \%$ were abnormal. In this study, positive predictive value (PPV) of pap smear is $74 \%$ and colposcopy is $80 \%$. The peak age group was between $41-50$ years. 
Conclusions: In this study, incidence of cervical intraepithelial neoplastic I (CIN I) was $48 \%$, CIN II $28 \%$, CIN III $20 \%$, squamous cell carcinoma $4 \%$. This emphasizes the use of all 3 methods PAP cytology (conventional method), colposcopy, and histology is complementary to each other and helps to reduce false negative cases. However, strategic approach to cervical screening should be followed considering the resource settings like SEE \& TREAT, SCREEN \& TREAT strategies.

\author{
Abstract \#86: Cervical cancer screening and HPV vaccine \\ acceptability among rural women in Rajnandgaon, Chhattisgarh: \\ Barriers \& obstacles \\ Meena Armo, Siddhi Sainik \\ BRLSABVM Govt. Medical College Rajnandgaon, Chhattisgarh, \\ India
}

Objectives: The study was undertaken to assess the major barriers that influence the utilization of cervical cancer screening among rural women of central India with the aim of helping health professionals to revise policies and practices.

Methods: It was a cross-sectional questionnaire-based study, conducted from January 2018 to December 2019 in the Department of Obstetrics and Gynecology at Government Medical College Rajnandgaon, the only tertiary care center of the district located in the southwest Chhattisgarh. A total of 897 women aged 21-65 years were included and assessed. Qualitative data were presented as frequencies and percentages by using SPSS version 21 .

Results: Of the total, $10.9 \%$ had heard of cervical cancer, while $6.8 \%$ had heard about cervical cancer screening. Unfortunately, only $0.6 \%$ women were screened by Pap test. None of them were ever heard of HPV vaccination. Although importance of screening and prevention had been thoroughly explained, despite the fact only $40 \%$ showed willingness to undergo cervical cancer screening in the future and HPV vaccination of their teenagers. However, it was very difficult to counsel and educate asymptomatic older population than symptomatic younger ones.

Conclusions: Intensive health education could be the major step to cross the major barrier of ignorance among rural population as well as healthcare provider's. There is also a need of community-based interventions to increase and promote HPV immunization coverage. Finally, subsidization will go a long way in lessening the financial burden, as well as increase utilization by rural population to change the current scenario.

\section{Abstract \#100: To compare the accuracy of various screening methods (VIA, VILI, Pap smear) of cervical cancer in a limited- resource setting}

Amrita Gaurav, Kavita Khoiwal, Jaya Chaturvedi, Anupama Bahadur All India Institute of Medical Sciences, Rishikesh, Uttarakhand, India

Objectives: To compare the accuracy of various screening methods (Pap smear, VIA, VILI and Colposcopy) in cervical cancer in a limited-resource setting and to assess the knowledge, attitude and practice of the study population towards cervical cancer screening. Methods: A total of 150 patients presenting to the gynaecology OPD at AIIMS Rishikesh were recruited from January 2019 till December 2019 and were sequentially screened with Pap smear, VIA, VILI and colposcopy. Suspicious lesions on colposcopy were subjected to cervical biopsy and KAP questionnaire was filled in the same sitting. Results: Pap test showed a sensitivity of $40 \%$, specificity $96.4 \%$, positive predictive value $44.4 \%$, negative predictive value $95.7 \%$ and diagnostic accuracy $92.67 \%$. For VIA sensitivity was $57.1 \%$, specificity $93.7 \%$, positive predictive value $30.7 \%$, negative predictive value $97.81 \%$, and diagnostic accuracy $92 \%$. For VILI sensitivity was $37.5 \%$, specificity $95 \%$, Positive predictive value $30 \%$, negative predictive value $96.4 \%$ and diagnostic accuracy $92 \%$. Only $4.6 \%$ had adequate knowledge, $11.3 \%$ were completely unaware of cervical cancer screening, $84 \%$ had suboptimal knowledge of cervical cancer screening. $62 \%$ showed favourable attitude towards screening and less than one percent were ever screened and none received the HPV vaccination.

Conclusion: All the three screening modalities Pap smear, VIA and VILI have similar diagnostic accuracy thus VIA and VILI can be used in resource-limited setting. The present study showed maximum sensitivity with VIA. It also showed that inadequate knowledge is the main barrier in utilisation of cervical cancer screening and vaccination services.

\section{Abstract \#166: Toolkit: Reaching The Unreached}

Shubhangi Mundhada

Geetai Nursing Home, Amravati, Maharashtra India

Objectives: To evaluate the impact of mobile cancer screening vehicle in screening for cancer cervix and cancer breast.

Methods: Cervical and Breast cancer is preventable by health education and early detection. Preventive services are sparsely available at community levels. We need to reach out to the under priviledged with the help of community /NGOs. For this purpose with the help of Rotary Club Of Amravati Midtown a van was designed to provide Pap smear collection and mammography testing services. The reading of Pap smears was done by cytology department Dr PDM medical College Amravati. Beginning from 2018 this van is visiting places and arranging screening camps all over India with the help of local Rotary clubs or other NGOs.

Results: From January 2018 we have covered all of Maharashtra, Madhya Pradesh, Chhattisgarh, Gujarat, Telangana reaching 31,943 women. 8102 mammography and 19,554 Pap smears were done. Out of these 657 abnormal Mammography and 1005 abnormal Pap smears were detected.

Conclusions

- Mobile van is a very good tool for attracting crowds.

- It provides good opportunity for health education with the help of slide shows, poster exhibition and speeches by local Gynaecologists.

- Linkages between smear collection and receiving reports in time needs to be streamlined.

- Active collaboration between FOGSI, ISCCP. IMA members and NGOS would prove meaningful.

- Follow-up and treatment of abnormal lesions is most important and will be strengthened.

We plan to introduce newer technology of VIA with mobile colposcope.

\section{Abstract \#209: Primary HPV Cervical Cancer Screening: Can we Triage Effectively to Limit Colposcopy Referrals and Overtreatment}

Sonia Mathai, Shrabanti Sarkar Ghosh, Barnali Ghosh, Jaydip Bhaumik

Tata Medical Centre, Kolkata, India 
Objective: To evaluate HPV test for primary cervical screening in identifying factors of HPV persistence. Identify feasible triaging strategies for colposcopy referral and follow-up in primary HPV cancer screening.

Design: Prospective analysis of 2502 women (25-65yrs) screened with primary HPV test and longitudinal follow-up from January 2018-February 2021 at TMC, Kolkata.

Results: A total of 253/2502 (10.1\%) women screened HPV positive and 226 (89\%) underwent colposcopy. On directed biopsies, $46.1 \%$ had no CIN, $44.6 \%$ had koilocytosis and CIN1, 7.5\% had CIN2 and $1.7 \%$ had CIN3 lesions. 232 (91.6\%) women with $\leq$ LSIL were observed and 175 were retested for HPV between 12 to 24 months, $72 \%$ women tested HPV negative and $28 \%$ remained HPV positive at a median of 12 months of repeat HPV test. 21/232 women underwent an ablative/excisional procedure and $100 \%$ (14) in this follow-up period tested HPV negative. For triage strategy for $\geq$ CIN2 according to the different genotype: for HPV 16 (21.3\%) the PPV was $11.1 \%$, HPV $18(14.6 \%)$ PPV was $13.5 \%$. The PPV for $\geq$ CIN2 for any HPV genotype, $16 \& 18$ and HPV $16 / 18 / 31 / 33 / 45 / 52 / 58$ was $8.3 \%, 11.6 \%$ and $9.3 \%$, respectively.

Conclusion: In resource-limited setting, though HPV screening is highly sensitive and specific, a single round of HPV testing may create a burden on colposcopy resources. Persistence of HR-HPV infections plays an important role in the development of cervical cancer, hence, both screening and triage at a single visit or stratification of risk will be an effective strategy to reduce colposcopy referrals.

\section{Abstract \#215: Cervical Cancer Screening in Malabar Region of Kerala: Lessons From Two Decades of Community-Based Screening}

Krishnanadha Pai, V C Ravindran, Harsha Gangadharan, Sujith Kumar Mullapally

Malabar Cancer Care Society, South Bazar, Kannur, India

Objectives: In the present study, we analyzed our experience in cervical cancer screening from 2002 to 2020 as part of communitybased screening.

Design: Retrospective analysis of prospective database of cervical cancer screening from 2002 to 2020 done by Malabar Cancer Care Society in Kerala.

Methods: The data on demography, type of screening, detection of precancerous and cancerous lesions was analyzed. Descriptive statistics were used to analyze the data.

Results: A total of 48,307 individuals were screened over 18 years. $55 \%$ were premenopausal. $33 \%$ were fully asymptomatic and remaining $66 \%$ had white discharge per vaginum $(25 \%)$, bleeding per vaginum $(14 \%)$ or combination of symptoms (25\%). $18 \%$ had abnormal per speculum findings (bleeds on touch, discharge, etc.) $86 \%$ had unhealthy cervix.26\% (12,650) had VIA/VILI and $74 \%$ $(35,658)$ had pap smear. The pap smear findings were normal $(41 \%)$, atrophic (17\%), inflammatory (40\%) and $2 \%$ had pre-cancerous/ cancerous changes. Of these lesions, $46 \%$ were HSIL, $36 \%$ were LSIL, $5 \%$ ASUS and $13 \%$ were squamous cell carcinoma (52 cases). $0.04 \%$ had invasive carcinoma detected by colposcopic biopsy after VIA/VILI whereas $0.015 \%$ was detection rate with pap smear. Conclusion: Cervical Cancer screening in large scale in low-resource setting is feasible in India by both pap smear and VIA/VILI.
Abstract \#231: Learning From Maternal Mortality Reduction Strategies to Reduce the Burden of Cervical Cancer in LowResource Settings

Ishak Kayode Lawal, Aliyu Kabir Suleiman, Kehinde Kazeem Kanmodi, Kadiri Michael Rotimi

Department of Obstetrics and Gynaecology, Federal Medical CenterBirnin-Kebbi, Kebbi State, Nigeria, End Cervical Cancer Nigeria Initiative, Birnin Kebbi, Nigeria, Department of Pathology, Federal Medical Center, Birnin Kebbi, Nigeria, Cephas Health Research Initiative, Ibadan, Nigeria. MEDBOOM Empire, Lagos, Nigeria.

Background: Cervical cancer is a disease of inequality, with lowresource settings bearing the highest burden of the disease. More than $80 \%$ of new cases and $90 \%$ of cervical cancer-related deaths occur in low-resource settings. Cervical cancer patients in low-resource settings typically present late; hence, the observed high case-fatality ratio. Understanding the factors responsible for the late presentation will inform evidence-based planning of interventions to promote early diagnosis while advocating for national screening program to reduce the incidence.

Objective: To adopt best practices used in maternal mortality reduction strategies to cervical cancer prevention strategy in lowresource settings.

Methods: A multi-staged mixed methods study of factors responsible for late presentation of cervical cancer patients in Nigeria modelled on the well-documented reasons for maternal mortality in low-resource settings. A hypothetical clinical course of a typical patient presenting late at Federal Medical Centre, Birnin-Kebbi, was constructed using combined history of -patients with confirmed cases of cervical cancer that presented to the hospital from January 2020 to January 2021. Factors causing late presentation were listed and were modelled using the delay concept of maternal motility.

Results: There are striking similarities between the factors causing late presentation of cervical cancer patients and causes of maternal mortality in low-resource settings. Both are preventable, associated with low socio-economic status, related to inequality in access to quality healthcare and have evidence-based preventive interventions. Also, these interventions have successfully been implemented in developed countries while developing countries have not been able to take advantage of these proven interventions to reduce their burden of these conditions.

Conclusion: The causes of late presentation of cervical cancer patients can be categorized into the delay model of causes of maternal mortality with potential of promoting better understanding of causes of late presentation of cervical cancer patients in low-resource settings. This knowledge can be applied to downstage cervical cancer thereby improving clinical outcome of management in low-resource settings.

\section{Abstract \#246: To Study the Accuracy of Pap Smear, Visual Inspection of the Cervix with Acetic Acid and Lugol's Iodine in Detecting Pre-Cancerous Lesions of the Cervix}

Sahana Girish, Nagarathnamma R, Mundlur Sirisha

Raja Rajeshwari Medical College and Hospital, Bengaluru, India

Objectives: To calculate the accuracy of Pap smear, VIA, VILI in detecting precancerous lesions of cervix, reference standard being biopsy cervix. The objective was to calculate the sensitivity, specificity, positive predictive value, negative predictive value of these tests and compare the results.

Methods: This cross-sectional study for a period of 18 months was carried out at Raja Rajeshwari Medical College and Hospital, 
Bengaluru for a sample size of 100. Cervix was closely inspected for macroscopic abnormalities. Pap smear was taken, and then visual inspection using 5\% acetic acid was done. After VIA, Lugol's iodine was applied to the cervix and naked eye examination was done. Careful examination of cervix and the transformation zone was carried out. All women were subjected for colposcopy-directed biopsy and HPE. Colposcopy-directed biopsy result was taken as gold standard. The results of Pap smear were compared with VIA and VILI findings taking colposcopy-directed biopsy as gold standard.

Results: The sensitivity calculated for visual inspection methods was $100 \%$ and specificity $62.5 \%$, positive predictive value $10 \%$ and negative predictive value $100 \%$. The sensitivity for Pap smear in this study was $75 \%$, specificity $96.8 \%$, positive predictive value $50 \%$ and negative predictive value $98.9 \%$.

Conclusion: Sensitivity for visual inspection studies was greater than that of Pap smear. Thus, in a low-resource country like ours where adequate number of cytologists and facilities are not available, visual Inspection with Acetic acid and Lugol's iodine can be used as an effective screening technique for cervical cancer.

\section{Abstract \#261: Evaluation of VIA and VILI as a screening tool in low-resource setting}

Shuchi Agrawal, Renu, Sangeeta

Department of Obstetrics \& Gynaecology, KGMU, Lucknow, India

Background: Lack of availability and high cost of cytology, primary HPV-based screening has led WHO, to recommend VIA and VILI for screening of cervical cancer in poor resource settings.

Methods: 210 women were screened with VIA, VILI in camp setting in remote area of Uttar Pradesh. Demographics details, awareness of women about cervical cancer and barriers for screening were recorded. Sensitivity, specificity and positive predictive value of each test calculated.

Results: Out of 210 women screened, $29(13.81 \%)$ were VIA positive, and remaining 181 women $(86.19 \%)$ had normal VIA,24 patients $(11.43 \%)$ were VILI positive and remaining 186 women $(88.57 \%)$ had normal VILI. Screen positives were called at tertiary care for Colposcopy and if required biopsy. Of the 29 VIA-positive cases, 16 $(55.17 \%)$ had CIN on HPE $3(15.79 \%)$ patients who had negative VIA but positive on VILI were reported to have positive HPE. Of the 24 VILI-positive cases, 17 (i.e., 70.83\%) had CIN on HPE and 2 cases $(10.53 \%)$ that were missed on VILI but positive on VIA had positive HPE. The sensitivity, specificity, positive predictive value and negative predictive value for VIA $(84.20 \%, 55.20 \%, 55.2 \%, 84.20 \%)$, VILI $(89.5 \%, 75.9 \%, 70.80 \%, 91.7 \%) .83 \%$ women were not aware about cervical cancer and its sign and symptoms,79\% reported lack of awareness, availability as barrier to screening and 63\%reported fear and anxiety as barrier to screening.

Conclusions: In a resource-constrainted setting VIA and VILI can prove a very helpful tool for screening and simultaneously it gives opportunity to interact with underprivileged women and impart information, Education and Counselling about cervical cancer and its prevention.

\section{Abstract \#265: Comparative study of effectiveness of per speculum, visual inspection of cervix with Acetic Acid (VIA) and Lughol's Iodine (VILI) versus conventional Pap smear as screening test for cancer cervix in presence of infection}

Deepika H K, Tamkin Khan, Nazia Ishrat, Kiran Alam, Fatima Khan Department of Obstetrics and Gynaecology, J.N.MC.H, Aligarh, India
Introduction: Worldwide, cervical cancer is the fourth most common cancer in women. $80 \%$ of the cases are from the developing countries. Various alternative strategies such as unaided visual inspections of the cervix after the application of 3-5\% acetic acid (VIA) and visual inspection after the application of Lugol's iodine (VILI) have been developed for screening in developing countries. However, the efficacy of these methods is in the presence of genital infection needs to be evaluated.

Objectives: To compare the sensitivity, specificity, positive predictive value, negative predict value of Per speculum examination, VIA, VILI, Pap smear in detecting premalignant and malignant lesions of cervix in the presence of infection.

Methods: This was a cross-sectional, comparative study in which 330 patients attending gynaecological OPD were enrolled. Per speculum examination, cervical swab for culture followed by Pap smear, VIA, VILI was done. Colposcopy and biopsy confirmation was done if tested positive by any of the above methods.

Results: Of the 330 patients, $132(40 \%)$ patients had cervical culture positive. Among the culture positive $122(92.4 \%)$ had positive per speculum, $21(15.9 \%)$ had positive Pap test, $36(27.2 \%)$ had positive VIA and 38 (27.9\%) had VILI positive. On biopsy 17 had CIN, 2 had squamous cell carcinoma. Sensitivity, specificity, positive predictive value and negative predictive value of Per speculum was $78.9 \%$, $5.3 \%, 12.5 \%, 60 \%$, respectively, for Pap they were $73.5 \%, 93.8 \%$, $70 \%, 95.4 \%$, respectively, for VIA they were $78.94 \%, 81.41 \%$, $41.6 \%, 95.8 \%$, respectively, and for VILI they were $78.94 \%, 80.53 \%$, $40.54 \%, 95.8 \%$, respectively.

Conclusions: VIA and VILI are effective screening tests even in the presence of genital infection.

\section{Abstract \#268: Reaching the Unreached-My Experience with Cervical Cancer Screening in a Low-Resource Setting}

Manoj B. Agalawe, Meera Agalawe

Bhandara, Maharashtra, India

India is a country with large variation in social, religious, cultural and resource access.

However, due to the intimate nature of the examinations and due to the cost involved, many candidates refuse screening in spite of the wide prevalence.

It requires a coordinated multidisciplinary approach involving primary healthcare workers, nurses, ASHA workers and doctors. The costs can be reduced and the sensitivity of testing improved by an algorithm that combines the use of LBC with HPV DNA detection and confirmation of type with PCR where required.

\section{Abstract \#274: Feasibility and Acceptability of Repeated Rounds} of Cervical Screening in Semi-Urban Women

Swati Tomar, Shachi Vashist, Sandeep Mathur, Jayashree Natarajan, Sarita Kumari, Anju Singh, Neerja Bhatla

Department of Obstetrics and Gynaecology, All India Institute of Medical Sciences New Delhi

Objective: To assess factors affecting participation and outcomes of a second round of cervical cancer screening.

Design: Cohort study.

Methods: Women aged 30-59 years from a semi-urban area of Delhi who had been screened with cytology, HPV testing (HC2) and VIA were recalled for screening with HPV and VIA after 5 years. Their awareness about cervical cancer was assessed at both times. 
Results: Of 1007 women screened during round-1, 133 (13.9\%) women were screen positive on any test: cytology $\geq$ ASCUS, 54 $(5.3 \%)$; VIA +, 50 (4.9\%); HPV +, 54 (5.3\%). CIN1, CIN2 and CIN3 lesions were found in 18, 3 and 5 women, respectively. Among CIN2/ 3 cases, $6 / 8$ were detected by HPV test, $3 / 8$ by cytology and $2 / 8$ by VIA. All received treatment as per standard-of-care. Five years later, only $714(70.9 \%)$ women were still available for round-2 screening, of whom $298(41.7 \%)$ declined participation: on duty $(n=144)$, said repeat screening is not required $(n=102)$, husband refused $(n=8)$, no reason $(\mathrm{n}=44)$. In round-2, $61(14.6 \%)$ women were screenpositive on any test; VIA + , $49(11.7 \%)$; HPV,$+ 24(5.7 \%)$. Of 133 screen-positive women of round-1, only 43 (32.3\%) underwent screening. Of previously diagnosed CIN 1 and CIN2/3, only $4 / 18$ and $4 / 8$ participated in screening, only one was found to have CIN1. New CIN3 lesions were diagnosed in 2 women in round-2, both were positive on VIA and HPV.

Conclusions: Repeated rounds of screening are not feasible or acceptable. A single round of screening with primary HPV testing will be the most effective screening strategy.

\section{Abstract \#287: Community-Based Cervical Cancer Screening Of 48,000 Females Over Last Two Decades: An Experience From Kerala}

Krishnanadha Pai, V C Ravindran, Harsha Gangadharan, Sujith Kumar Mullapally

Malabar Cancer Care Society, Kannur, Kerala, India

Objective: In this study, we analysed our experience in cervical cancer screening from 2002 to 2020 as part of community-based screening.

Design: Retrospective analysis of prospective database of cervical cancer screening from 2002 to 2020 was done by Malabar Cancer Care Society in Kerala.

Methods: The data on demography, type of screening, detection of precancerous and cancerous lesions were analysed. Descriptive statistics were used to analyse the data.

Results: A total of 48,307 individuals were screened over 18 years. $55 \%$ were premenopausal. $33 \%$ were fully asymptomatic and remaining $66 \%$ had white discharge per vaginum $(25 \%)$, bleeding per vaginum $(14 \%)$ or combination of symptoms $(25 \%) .18 \%$ had abnormal per speculum findings (bleeds on touch, discharge, etc.) $86 \%$ had unhealthy cervix.26\% (12,650) had VIA/VILI and $74 \%$ $(35,658)$ had pap smear. The pap smear findings were normal $(41 \%)$, atrophic $(17 \%)$, inflammatory $(40 \%)$ and $2 \%$ had pre-cancerous/ cancerous changes. Of these lesions, $46 \%$ were HSIL, $36 \%$ were LSIL, 5\% ASUS and 13\% were squamous cell carcinoma (52 cases). $0.04 \%$ had invasive carcinoma detected by colposcopic biopsy after VIA/VILI whereas $0.015 \%$ was detection rate with pap smear. Conclusion: Cervical Cancer screening in large scale in low-resource setting is feasible in India by both Pap smear and VIA/VILI.

\section{Abstract \#315: Lessons Learnt from 'Screen and Treat' Strategy- Based Cervical Cancer Screening Demonstration Project in Rural India}

Manisha Vernekar, Dipanwita Banerjee, Puja Chatterjee, Chandrima Ray, Sreeya Bose, Ranajit Mandal

Department of Gynaecological oncology, Chittaranjan National Cancer Institute, Kolkata, India

Objectives: The strategies to prevent cervical cancer should be country specific, affordable and feasible in the population. The community-based project primarily assessed the utility of "Screen and Treat" strategy by using low-cost VIA method with 5\% acetic acid and /or HPV DNA detection test. Secondary objective was to evaluate the response and compliance of the participants.

Methods: A prospective study (approved by Institutional Ethics Committee) was conducted by the Department of Gynaecological Oncology, Chittaranjan National Cancer Institute (CNCI) from January 2016 to April 2017. Eligible women were tested for HPV DNA test and/or by VIA with $5 \%$ acetic acid. VIA positive women were treated by either Cryotherapy or thermal ablation following a punch biopsy at same visit. Rest were referred to CNCI for excision. The HPV-positive women were recalled for VIA followed by biopsy and treatment. The results were analysed by Stata version 14 .

Results: A total number of 6592 women were recruited with $4.96 \%$ VIA positive and $4.24 \%$ HPV DNA positive. Colposcopy and biopsy followed by treatment was done in $82.1 \%$ women. Acceptability of Thermal ablation was higher than Cryotherapy with less pain (3.1\% vs $6.6 \%$ ). Women who were exposed to unnecessary treatment was $61.9 \%$. Only $43.1 \%$ women had their follow-up.

Conclusion: In a resource-constrained setup, utilisation of available resources with cost-effective strategies like "Screen and Treat" can help to reduce the burden of cervical neoplasia with increased chances of overtreatment.

\section{Abstract \#318: Feasibility Of Self-Sampling For HPV DNA Detection In A Community-Based Cervical Cancer Screening Project}

Puja Chatterjee, Chandrima Ray, Sreeya Bose, Dipanwita Banerjee, Manisha Vernekar, Ranajit Mandal

Department of Gynaecological Oncology, Chittaranjan National Cancer Institute, Kolkata, India

Objective: Paucity of resources and trained professionals makes it difficult to implement a cervical cancer screening program in India. This study aims to evaluate the feasibility of self-sampling for detection of human papillomavirus (HPV) DNA in a communitybased cervical cancer screening project.

Methods: Women (30-60 years) were assigned to do self-sampling or get their samples collected by healthcare workers in outreach clinics. The samples were brought to the institute and were tested by hybrid capture2 (HC2) test for 13 high-risk HPV types. HC2 positive women were brought to the institute where they underwent colposcopy, biopsy and treatment by Thermal ablation, Cryotherapy or Loop Electrosurgical Excision Procedure (LEEP). A focussed group discussion was done with health workers involved with this project in the form of a questionnaire.

Results: From May 2017 and December 2020, 15,311 women were recruited. Amongst them, 4916 (32.1\%) had self-sampling and 10,395 $(67.9 \%)$ had health-worker collected sample. The HC2 positivity rates in both groups were $269(5.5 \%)$ and $652(6.3 \%)$, which was not significantly different statistically $(\mathrm{P}=0.06)$. The colposcopy rates and Cervical Intraepithelial Neoplasia (CIN) 2 and 3 detection rates were also similar. All women were comfortable with self-sampling with no sample inadequacy or wastage of collection kits. The health workers rated both procedures as acceptable. The advantage of selfsampling was that no examining table or light source was required and the screened women were less "shy" while sample collection. Conclusion: Self-sampling for HPV may increase participation in cervical cancer screening programs, especially in the COVID19 era. 
Abstract \#327: Cervical Precancer Lesions Seen in Women Screened with Visual Inspection with Acetic Acid and Lugol's Iodine: A Population-Based Study in Gombe, North-East Nigeria

Halima Usman Farouk, Olakunle Azeez, Amina Muhammed, AliGombe Musa, Aliyu Ibrahim Lawan, Bala Muhammed Audu

Gombe State University/ Federal Teaching Hospital, Gombe, Nigeria

Background: Cervical cancer is the second most common cancer in women in Sub Saharan Africa with 570,000 women diagnosed with cervical cancer in 2018. Eighty-five percent of these occur in low and middle-income countries, with 311,000 women dying from the disease worldwide. Nigeria has recorded a 5-year prevalence of $21.6 \%$ with 10,000 new cases and 8,000 deaths annually. With the launch of the WHO Global Strategy to Accelerate the Elimination of Cervical Cancer in November 2020, it became pertinent to employ the use of screen and treat with Visual inspection with acetic acid and Lugol's iodine (VIA/VILI) in low-resource setting. Perhaps successful implementation of wide screening and treatment of precancerous lesions could reduce the number of new cases of the disease and related deaths in Gombe.

Aim: To determine the prevalence of VIA/VILI positive cervical lesions among 1000 women screened for cervical precancer lesions in Gombe.

Methods: This was a cross-sectional study of women who attended the free cervical precancer screening with VIA/VILI in Gombe from $11^{\text {th }}$ of January, 2021 to $10^{\text {th }}$ of February, 2021 at Federal Teaching Hospital Gombe.

Results: One thousand women had speculum examination of the vagina and 954 women had visual inspection of the cervix with acetic acid \pm Lugol's iodine. Of these $222(22.3 \%)$ women had VIA/VILI positive lesions of the cervix. Three women had frank invasive carcinoma of the cervix. The age range was between $30-80$ years. The mean age was 40.35 years \pm 8.28 years. The mean parity was $3.74 \pm 2.98$ children. The average age at coitarche was $20.97 \pm 5.32$ years.

Conclusion: VIA/VILI positive lesions of the cervix are quite common among women 30 years and above in Gombe and implementation of screening with "See and treat" can reduce cervical cancer incidence and its mortality.

\section{Management of CIN}

\section{Ablative Methods \\ Paper number \#295: A Pilot on the Use of Thermal Ablation and Via to Eliminate Cervical Cancer in Kebbi State And Fct, Nigeria}

Zainab Shinkafi-Bagudu, Dr. Aminu Aliyu, Hadiza Amanabo-Arome

Medicaid Cancer Foundation, Nigeria

Background: Low-and Middle-Income Countries (LMICs) are the worst hit by cervical cancer mortality. Routine screening is effective for treatment and reduction of related deaths. Common screening methods such as cytology-based histological and genetic typing confirmation of cervical intraepithelial neoplasia are difficult and expensive to carry out in LMICs. However, the use of thermal ablation machines, due to its effectiveness, simplicity, portability, and Visual Inspection using Acetic Acid (VIA) is convenient for remote health facilities. The WHO global strategy of 90-70-90 to eliminate cervical cancer by 2030 requires such affordable techniques.

Objectives of the training were to assess and increase knowledge of the use of thermal ablation machine and VIA for screening; boost the capacity of health workers; train the health workers to identify precancerous lesions and contribute to reduction in cancer burden in Nigeria.

Method: We collected data from ninety Healthcare workers in Kebbi State and FCT, Nigeria. The sample population consisted of CHEWs, nurses, and doctors. Using structured questionnaires and surveys, the study had pre- and post-training assessments.

Result: There was a gap in the knowledge of cervical cancer treatment between health professionals and CHEW. Trainees learnt how to use thermal ablation machines and VIA to screen cervical cancer lesions. Post-test Mean result of participants from Kebbi was 70.54 while that of Abuja was 82.4.

Conclusion: The use of thermal ablation and VIA in the screening of cervical cancer lesions is easily accepted and absorbed by health workers and will accelerate the collective effort to curtail cervical cancer in Nigeria.

Keywords: Thermal ablation, VIA, cervical cancer.

\section{Abstract \#10: Cryotherapy-The Best Treatment For Symtomatic Cervical Ectopy}

Ajabrao Sakharam Vasu

Sai Nursing Home, Chikli, Buldana, Maharashtra, India

Objectives: To evaluate outcome of Cryotherapy on symptomatic Cervical Ectopy.

Method: A total of 150 women age between 24 to 40 years were studied. All had Cervical Ectopy on Colposcopy and their Pap Smear was taken. Out of these 140 had negative Pap Smear whereas, 10 had LSIL. All the 150 women were called separately and Cryotherapy on Colposcopy was performed. Cryotherapy consisted of the use of $\mathrm{N} 2 \mathrm{O}$ to destroy columnar epithelium by 3 min freeze, 5 min thaw and again 3 min freeze. Iceball was formed around Cryotip. The followup was done after 12 weeks and were re-examined after one year.

Result: There was an absence of Cervical Ectopy and Acitowhite area on Colposcopy. Only Nine women had persistant complaint of vaginal discharge. On follow-up, all LSIL women Pap Smear was negative. So, cure rate for Cervical Ectopy was $100 \%$ and for vaginal discharge was $94 \%$. No serious side effect was noticed. There was an increase in vaginal discharge for first 15 to 20 days after procedure. After 20 days, the rate of discharge gradually decreased.

Conclusion: Cervical Cryotherapy is a cheap, effective and simple procedure with $100 \%$ cure rate for Cervical Ectopy and $94 \%$ cure rate for vaginal discharge. Hence, we recommend that clinicians perform the procedure in such patient without much delay.

\section{Abstract \#27: Thermo-coagulation in a VIA-based 'Screen and Treat' approach to cervical cancer screening: Initial experience}

RukhiyaBegum, Glory Hyma, Yadamma CH, Pranitha J, Narayanamma $M$, Anuradha $P$

Institute for Rural Health Studies, Hyderabad, India

Objectives: Thermocoagulation (TC) is an acceptable and feasible method with similar treatment outcome to cryotherapy without some of its disadvantages. We report our experience with portable batteryoperated thermocoagulators (Liger) in the district-level screen and treat cervical cancer screening program in Telangana, India.

Method: VIA-positive women were identified among those screened in 2 district-level cancer screening clinics managed by 3 trained ANMs in each center from October 2018 to December 2019. The eligibility for ablative treatment was assessed and the women were 
offered treatment by TC. Women willing were treated in the same visit or were recalled at a later date to suit their convenience. Posttreatment follow-up was done at 6 and 12 months.

Results: There were 823 VIA-positive women of which 153 underwent TC. About 90 of them were treated on the day they were screened. A total of 31 were with biopsy-proven cervical lesions indicating an overtreatment rate of 5 times. However, treatment of 33 women with high-grade lesions remains pending among those who were not treated during the same visit.

Conclusions: Thermocoagulation is both a useful and acceptable ablative method of treatment in programs following a 'screen and treat' protocol to ensure compliance in treatment of screen positive women. In remote rural areas, thermocoagulation far outweighs cryotherapy as nitrous oxide is frequently difficult to obtain and leaky cylinders are all too often the norm.

\section{Abstract \#32: Interim analysis of a prospective clinical trial of diathermy for patients with CIN2/3 from a single institution in Japan}

Takuma Fujii, Rina Kawahara, Ryoko Ichikawa, Yasumichi Miki, Hiroyuki Nomura, Iwao Kukimoto

Fujita Health University, Japan

Objectives: For prevention of cervical cancer, active intervention is recommended to target precursor lesions. Laser cone resection or vaporization is the best option in very high-income countries, but the equipment is still very expensive and its use is limited in Japan. Diathermy offers an alternative less expensive approach. Here we present an interim analysis of the efficacy of diathermy in a prospective clinical trial.

Methods: Patients with CIN2 $(n=32)$ or CIN3 $(n=6)$ were referred to Fujita Health University Hospital in Japan from November 2016 through December 2019. All 38 patients, aged 29-49 years (median age 35.5) were followed up. Diathermy (Sabre Genesis, CONMED NY, USA) was performed under local anesthesia. Patients will be followed up to 24 months according to the scheduled protocol.

Results: Before surgery, 33 of $34(97 \%)$ patients were HPV-positive, 10 had more than one type of HPV and 23 a single type. After surgery at 12 months, 23 of $25(92 \%)$ patients were classified as negative for intraepithelial lesions or malignancy (NILM). Eight of 25 (32\%) were HPV-positive, and 7 of these $(87.5 \%)$ were NILM. Six of the latter were infected with a single HPV type and the remaining patient with two.

Conclusions: Approximately one-third of patients were infected with multiple HPV types before treatment. At 12 months, most HPVpositive patients were infected with a single type; $32 \%$ classified as NILM were HPV-positive. The final histological diagnosis at 24 months is eagerly anticipated.

\section{Abstract \#117: Cold Coagulation \& Cancer: is there a correlation?}

Kalpana Ragupathy, Lauren Crossley, Sheila Nicoll

Ninewells Hospital, United Kingdom

Objectives: To evaluate incidence of Cervical Intraepithelial Neoplasia (CIN) and historical use of Cold Coagulation (CC) in women with cervical cancer in our unit.

Methods: A retrospective cohort study design was utilised to evaluate incidence of CIN and historical use of CC in all women diagnosed with cervical cancer in our Colposcopy unit within a 19 -year period (1997-2015). Women diagnosed with cervical cancer within the study period with a history of past CIN were identified. Type of treatment received for historical CIN was then identified using 4 data sources: SCRRS, cervical cancer audit database, pathology database and colposcopy cards. Time from CIN treatment to cervical cancer diagnosis and grade of CIN treated were identified.

Results: Of 464 women diagnosed with cervical cancer within the study period, 50 had past history of CIN (10.78\%). 21 (17 high-grade CIN) were treated with coagulation, 16 with LLETZ (14 high-grade CIN) and 3 (3 high-grade CIN) with both. Mode of treatment was not identifiable in 10 women. Mean time from treatment with $\mathrm{CC}$ to cancer diagnosis was 124 months while mean time from treatment with LLETZ to cancer diagnosis was 68 months.

Conclusions: Our data suggest that historical CIN is not uncommon in women diagnosed with cervical cancer. Average time from treatment of CIN to cancer diagnosis was greater in the cold coagulation group versus LLETZ group. It can be inferred that CC is a safe and effective treatment for CIN and association with cervical cancer is no more than LLETZ.

\section{Abstract \#123: Success of Cold Coagulation as a treatment modality of Cervical Intraepithelial Neoplasia-20 year follow-up study}

WendyMcMullen, Kalpana Ragupathy, Vimala Nellore, Thummini Jayasinghe, Natasha Tzortzidis, Ayesha Mujahid

NHS Tayside, United Kingdom

Objectives: Cold coagulation (CC) is an ablative method of treatment of Cervical Intraepithelial Neoplasia (CIN). CC is simple, safe, low cost and easy to teach with high cure rates, and, importantly no impact on future fertility or pregnancy. However, there has been a reluctance to adopt this method widely because of paucity of long-term followup data. Our study was undertaken to address this.

Methods: A database was created of all women treated with $\mathrm{CC}$ for high-grade CIN in a single Scottish Unit between 1996 and 1998 $(\mathrm{n}=883 ; \mathrm{n}=322$ for CIN 2 and $\mathrm{n}=561$ for CIN3). Cytology and pathology results were collected over a 20 -year follow-up period until 2018. Long-term outcome measures were cytoloreversion, retreatments required and cancer occurrence.

Results: $881 / 883(99.7 \%)$ had type 1 or 2 Transformation Zone. There was evidence of endocervical gland or crypt involvement in 287/883 (32\%) women. Cytoreversion (normal cytology) for CIN2 and CIN3 was $92 \%$ and $96 \%$ at one year, $95 \%$ and $97 \%$ at 2 years, $96 \%$ and $99 \%$ at 5 years, $96 \%$ and $98 \%$ at 10 years and $91 \%$ and $97 \%$ at 20 years. $16 / 883(1.8 \%)$ women had recurrence of high-grade CIN in the follow-up period (18 months to 693 months, average 338 months). 12/16 women underwent treatment with LLETZ. One patient developed cancer (CIN3 group) 76 months after CC treatment. Conclusions: Our study shows that Cold Coagulation is a safe treatment which can effectively treat high-grade CIN with excellent long-term outcomes.

Abstract \#164: Comparison of Provider and Client Acceptability of Thermocoagulation and Cryotherapy for VIA Positive Cervical Lesions

Manju Lata Verma, Uma Singh, Parul Sharma

Department of Obstetrics and Gynaecology, King George Medical University, Lucknow, India

Objectives: Our objective was to assess the acceptability of thermocoagulation amongst the providers as well as clients and 
comparing the acceptability and efficacy of the same with cryotherapy.

Methods: It was a randomized controlled trial where VIA was performed as screening test on 30-50-year-old women attending Gynae OPD. VIA-positive women were randomized to receive either cryotherapy or thermocoagulation. Provider and client acceptability was assessed immediately after procedure using IARC validated questionnaire. At 6 weeks and 6 months follow-up, women were evaluated for any side effect and treatment efficacy.

Results: Of total 880 women screened, 104 were VIA positive. Only 92 women were eligible for ablative therapy, out of which 46 received cryotherapy and 46 received thermocoagulation. Compared to cryotherapy, significantly higher proportion of providers found thermocoagulation very easy to use and set up, were satisfied with its probe length and shape and did not face any technical problems while performing it. The mean patient satisfaction score for thermocoagulation was significantly higher than cryotherapy $(9.33 \pm 0.99 \mathrm{v} / \mathrm{s}$ $8.50 \pm 1.43, \mathrm{p}=0.002$ ). Higher proportion women who were treated with thermocoagulation reported no side effect immediately after procedure and at 6 weeks follow-up.

Conclusion: With similar efficacy as cryotherapy, thermocoagulation offers better provider and client acceptability in terms of ease of use, portability, less technically demanding, shorter treatment and lesser side effects and it can be a more suited and feasible treatment modality for cervical precancerous lesions in low-resource settings.

\section{Abstract \#243: Effectiveness and Safety of Thermal Ablation in Treating Cervical Intra Epithelial Neoplasia in a Low- Resource Setting}

Rema P, Suchetha S, Dhanya Dinesh

Regional Cancer Center, Trivandrum, Kerala, India

Objective: Thermal ablation is an ablative technique used in the treatment of CIN (cervical intraepithelial neoplasia). In this study, we looked into the effectiveness of thermal ablation in treating CIN of all grades and the side effects and complications associated with the procedure.

Design: Retrospective observational study.

Methods: We included 50 patients who underwent thermal ablation in our institution in the study. Patients diagnosed with all grades of CIN underwent thermal ablation after colposcopic evaluation which ensured complete visualization of transformation zone and excluded lesions suggestive of invasive carcinoma. Multiple overlying applications with the thermal ablator was performed with temperature set at 100 degree. Side effects and complications during and after procedure were documented. Patients were followed up at 6 weeks, 6 months and 1 year after the procedure.

Results: In our study, we achieved a follow-up rate of 94\%. 40 (80\%) were cured of CIN. Cure rates were $94.6 \%$ for CIN1, $60 \%$ for CIN2 and $25 \%$ for CIN 3 disease. Cure rates were significantly associated with grade of CIN and Reid score. All patients tolerated procedure well. $18 \%$ of patient's complained of mild pain during procedure, and none required any intervention. There were no complications during procedure. Eighteen patients $(36 \%)$ developed mild side effects and three patients $(6 \%)$ developed complications at one year. Only one patient $(2 \%)$ needed admission and treatment for bleeding secondary to local infection.

Conclusion: Thermal ablation is a safe and effective technique for low-grade CIN. Its efficacy in high-grade CIN needs further prospective evaluation.

\section{Subtheme: Excisional Methods}

Abstract \#48: Cervical excisional treatment, HPV infection and risk of preterm delivery - a Swedish population-based study

Johanna Wiik, Staffan Nilsson, Cecilia Kärrberg, Björn Strander, Bo Jacobsson, Verena Sengpiel

Department of Obstetrics and Gynaecology, Institute of Clinical Sciences, Sahlgrenska Academy, Gothenburg University, Sweden

Objectives: Excisional treatment for cervical intraepithelial neoplasia (CIN) is associated with preterm delivery (PTD) including preterm prelabour rupture of the membranes (pPROM). It has been shown that also untreated CIN is associated with PTD. We aimed to explore the magnitude of these associations and other adverse obstetrical outcome.

Methods: Retrospective register-based study comprising all women with singelton deliveries in Sweden 1999-2016. Data were retrieved from the Medical Birth Registry and the Swedish National Cervical Screening Registry. Women with normal cytology all life ( $n=340,290)$ were compared to a) women with sign of HPV infection close to pregnancy (abnormal cytology $(n=11,936)$ or positive HPV-test $(\mathrm{n}=2587))$, b) women treated for CIN before delivery $(\mathrm{n}=25,123)$ and $\mathrm{c}$ ) women with $\mathrm{CIN} /$ carcinoma diagnosed after delivery $(n=34,251)$ by logistic regression adjusted for socioeconomic and health-related confounders.

Results: PTD was increased in all groups compared to women with normal cytology all life $(4.7 \%)$, with greatest risk for the treated group (9,0\%, OR $1.80(1.71-1.89))$, but also for women with HPV infection close to pregnancy $(5,9 \%$, OR $1.19(1.10-1.29)$ and with CIN/carcinoma after delivery (6,1\%, OR 1.10 (1.04-1.16)). Paired analysis in 5547 women with a delivery both before and after treatment showed increased risk of PTD after treatment, OR 1.27 (1.07-1.50). The treated group had increased risk also for pPROM (OR 2.25 (2.08-2.44)) and chorioamnionitis (2.44 (2.05-2.90)). HPV infection close to pregnancy was associated with pPROM (OR 1.22 (1.06-1.40)).

Conclusions: Women with HPV infection close to pregnancy have a small increased risk for PTD and pPROM, while excisional treatment increases risks further.

\section{Abstract \#50: Evaluation of factors affecting margin positivity and persistent disease after LEEP for CIN}

Anila Tresa, Rema P, Suchetha S, Dhanya Dinesh

Regional Cancer Centre, Trivandrum, India

Objectives: To assess the risk factors associated with positive margin and persistent disease after LEEP for CIN.

Method: A retrospective audit of the 156 patients who underwent LEEP, from 2011-2018 was done. Case records of patients were analyzed for socio-demographic data, colposcopy details, dimensions of leep specimen (diameter, volume, size), histopathology (margin positivity, grade), follow-up data. Persistent disease was histopathologically confirmed by repeat LEEP and hysterectomy. Data was analysed with SPSS software and significant association was found using chi-square test.

Results: $49 \%$ patients had a Type 3 transformation zone.71.2\% had high-grade colposcopic lesions. Margin positivity was noted in $33.3 \%$ patients. $24.4 \%$ patients had endocervical margin positive. Persistent disease was found in $89 \%$ of patients with positive margin. There was significant association between margin positivity and high-grade colposcopic scoring $(\mathrm{p}$ value $=0.000$ ) type of leep (single pass $\mathrm{p}$ value 0.046 ). There was no association between margin positivity and diameter, volume, depth of cone, type of transformation zone. There 
was significant association between residual disease and postmenopausal status (pvalue $=0.012)$, margin positivity $(\mathrm{p}$ value $=0.004)$, high-grade colposcopy score. $($ pvalue $=0.00)$. However, no association was found between the depth, radius or volume of cone, type of transfomation zone.

Conclusion: Dimensions of the conisation specimen is not predictive risk factor for margin positivity and residual disease. Postmenopausal women, high-grade lesions, and positive endocervical involvement are important predictive factors for margin positivity and also residual disease. Hence we should be careful in treatment of these patients and also keep them on regular follow-up.

\section{Abstract \#55: Local cervical excisional treatment, cone length and risk of preterm delivery-a Swedish population-based study}

Cecilia Kärrberg, Johanna Wiik, Staffan Nilsson, Björn Strander, Bo Jacobsson, Verena Sengpiel

Sahlgrenska University Hospital, Sweden

Objectives: The exact impact of treatment for cervical intraepithelial neoplasia (CIN) on the risk of subsequent preterm delivery (PTD) is not known. The aim of this study was to explore the effect of treatment of cervical pre-invasive and early invasive disease on PTD and other adverse pregnancy outcomes and to investigate how this risk may be modified by cone lenght and comparison group.

Methods: A retrospective cohort study comprising all women with singelton deliveries in western Sweden 2008-2016. Data were retrieved from the Swedish Medical Birth registry and The Swedish Cervical Screening Registry. Women were divided into groups: Treatment of CIN before delivery, untreated CIN before delivery and women with normal cytology all life and compared with logistic regression adjusted for socioeconomic and health-related confounders. The cone length was registered after excisional treatment before fixation. Association between cone length and gestational age was analyzed with Pearson correlation and Chi-square test.

Results: Treated women $(n=2486)$ had an increased risk for PTD compared to women with normal cytology $(\mathrm{n}=57,036) ;(6.7 \%$ vs $4.3 \%$, OR $1.55(1.31-1.83))$. A total of 2262 treated women had records of cone lenght and $159(7 \%)$ of these had a PTD. There was a correlation between cone lenght and gestational age at birth. Cone lenghts of $10 \mathrm{~mm}$ or more increased the risk for PTD, compared to cone lengths less than $10 \mathrm{~mm}$ OR 1.77 (1.28-2.44). Additional data under preparation will be presented at the conference.

Conclusions: Excisional treatment of CIN and cone lenght increase the risk of PTD.

\section{Abstract \#64: Risk of high-grade histopathology diagnosed by cervical conization in endocervical curettage CIN1: a case- control study}

\section{Qing Cong}

Obstetrics and Gynecology Hospital of Fudan University, China

Objectives: The role of endocervical curettage (ECC) cervical intraepithelial neoplasia 1 (CIN1) in colposcopy-directed biopsy is controversial. We aimed to estimate the risk of CIN $2+$ on loop electrosurgical excisional procedure (LEEP) specimen in ECC CIN1 compared with ectocervical CIN 1 and negative ECC finding (ECC; CIN1 vs biopsy CIN1).

Methods: A retrospective computer-based search was performed. Subjects were enrolled from January 2013 to December 2018 in Obstetrics and Gynecology Hospital of Fudan University. Case group (ECC CIN1) comprised women with ECC CIN1 and concurrent biopsy CIN1/negative findings. Control group (biopsy CIN1) comprised women with biopsy CIN1 and concurrent negative ECC findings. Variables including age, cytology, hrHPV, ECC results were included in univariate and multiple logistic regression analysis and $\mathrm{P}<0.05$ was defined statistically significant.

Results: A total of 1195 ECC CIN1 and biopsy CIN1 who underwent LEEP were included in the study. ECC CIN1 group consists of 400 women, with LEEP histopathology results of 104 (26.00\%) CIN2 + . Biopsy CIN1 group consists of 795 women, with LEEP histopathology results of $150(18.87 \%)$ CIN2 + . Univariate logistic regression showed that cytology $(\mathrm{P}<0.001)$ and $\mathrm{ECC}(\mathrm{P}=0.005)$ results were significantly different between $<\mathrm{CIN} 2+$ and $\mathrm{CIN} 2+$. Multivariate logistic regression showed cytology of ASC-H $(\mathrm{OR}=4.73,95 \% \mathrm{CI}$ : 2.78-8.05, $\mathrm{P}<0.001)$ and HSIL $+(\mathrm{OR}=4.88,95 \% \mathrm{CI}: 3.00-7.94$, $\mathrm{P}<0.001)$, ECC CIN1 (OR $=1.80,95 \%$ CI: $1.33-2.44, \mathrm{P}<0.001)$ were risk factors of CIN2 +

Conclusions: ECC provided more information than biopsy for CIN1 but less information than high-grade cytology for risk stratification.

Abstract \#90: Long-term outcomes of women treated for HSIL at a University Hospital colposcopy unit in SA. A 5-year retrospective cohort study

Bothwell Takaingofa Guzha, Nondumiso Ngxola, Rakiya Saidu, Tracey Adams, Linda Rogers, Nomonde Mbatani

University of Zimbabwe, College Of Health Sciences, Zimbabwe

Objectives: This study was done to establish baseline data on the performance of the cytology and colposcopy-based cervical cancer screening programme at the Groote Schuur Hospital (GSH) colposcopy clinic.

Methods: Retrospective cohort study of all the women with HSIL Pap smears seen at GSH colposcopy clinic between 01/01/2010 and $31 / 12 / 2015$. The outcome measures were; LLETZ and cone biopsy complication rates, cure rate, treatment failure and invasive cervical cancer rates. Data were managed and analysed using IBM SPSS Statistics Version 25 and Microsoft Structured Query Language (SQL) version 2014.

Results: A total of 7601 women were referred to the GSH colposcopy clinic during the study period. $\geq$ HSIL were confirmed histologically in $74.1 \%(2282 / 3081)$ women. At the four-month follow-up visit, $61.2 \%(742 / 1213)$ of the women were considered cured, and $17.0 \%$ (206/1213) had persistent/residual disease. In women considered cured at 4 months, recurrence was very low, and it peaked at ten months at $1.5 \%(11 / 740)$. By 24 months, the cumulative recurrence rate was $4.6 \%(34 / 742)$. In women with persistent disease at the 4-month follow-up visit, only $0.5 \%$ (1/202) developed invasive cervical cancer. LLETZ and cone biopsy complication rate was $7.2 \%$ (117/1628). In a Cox-regression model, taking HAART was the only factor associated with a reduced risk of disease recurrence $(\mathrm{p}=0.0261)$.

Conclusions: LLETZ and cone biopsy are safe procedures. After cure, recurrence rates are low. In women who are treated for HSIL, cervical cancer is very rare. Taking HAART was associated with a reduced risk of disease recurrence.

\section{Abstract \#153: Impact of anesthesia mode, local versus general, on loop electrosurgical excision specimen}

Yossi Tzur, Roza Berkovitz Shperling, Ido Laskov, Dan Grisaru, Nadav Michaan

Lis Maternity Hospital, Sourasky Medical Center, Tel Aviv University, Tel Aviv, Israel 
Objective: To compare Loop Electrosurgical Excision Procedure (LEEP) specimen characteristics performed under local versus general anaesthesia.

Design: Observational cohort study.

Methods: A total of 161 patients who underwent LEEP between 2011 and 2019 at a single, tertiary medical centre were enrolled. Collected data included demographics, LEEP indication, cone dimension and margins' involvement as well as recurrence rate. The primary outcome was the rate of positive LEEP margins.

Results: Overall, 79 patients underwent LEEP under general anaesthesia and 82 patients under local anaesthesia. Demographic characteristics including age, BMI, nulliparity, and smoking rates were similar between groups. The rates of abnormal cytology and high-risk HPV subtypes prior to conization as well as the rate of higher risk pre-conization histologies (CIN II-III, SCC and adenocarcinoma) were comparable between groups. The rate of positive specimen margin was comparable between women who underwent LEEP under general anesthesia compared with local anaesthesia both in the endocervical margin $(22 / 77(28.5 \%)$ vs $16 / 80(20.0 \%) \%$, respectively, $P=0.21)$ and in the ectocervical margin $(24 / 75(32.0 \%)$ vs $23 / 80(28.8 \%), P=0.66)$.

During the first year after conization, the rates of abnormal PAP, the rate of women who underwent repeated conization, and the rate of high-grade dysplasia $(\mathrm{CIN} \geq 2)$ in repeated conization specimen were not significantly different between the general and local anaesthesia groups, $(17.2 \%$ vs $18.1 \%, P=0.93)$, $(9.6 \%$ vs $15.6 \%$, $P=0.38)$, (40.0\% vs $42.8 \% P=0.92)$, respectively.

Conclusion: The rate of involved specimen margin and postconization abnormal findings were comparable between women.

\section{Abstract \#173: Significance Of LEEP Specimen Dimension In Predicting Margin Positivity For CIN}

Anila Tresa Alukal, Rema P, Suchetha S, Dhanya Dinesh, Sivaranjith J, Aleyamma Mathew, Aswathy G Nath

Regional Cancer Centre, Thiruvananthapuram, Kerala, India

Objectives: Loop electrosurgical excision procedure (LEEP) is commonly used for treatment of high-grade CIN. A negative margin is needed to ensure treatment. The study aims to assess the significance of LEEP specimen dimension in predicting margin positivity and persistent disease after LEEP for CIN.

Design: Retrospective cohort study.

Methods: A total of 156 patients who underwent LEEP during 2011-2018 included in the study. We analysed the socio-demographic characteristics, colposcopy details, histopathology, dimensions of LEEP specimen. The thickness was measured from the surface to stromal margin and the length was measured from ecto to endocervical margin as defined in 2011 IFCPC nomenclature. In case of multiple passes, the mean value was taken. A cut-off to predict the chance of positive margin was calculated in premenopausal and postmenopausal women using Youden Index.

Results: Margin positivity was seen in $33.3 \%$ of patients. There was significant association between margin positivity and swede score of 5 and more, a high-grade lesion on IFCPC score, inner margin involvement, LEEP done in a single pass. The cutoff for margin positivity was length of $0.5 \mathrm{~cm}$ and thickness of $0.75 \mathrm{~cm}$ for premenopausal women. For postmenopausal women, who had a comparatively smaller cervix, the cutoff for length of specimen was $0.5 \mathrm{~cm}$ and thickness was $0.35 \mathrm{~cm}$.

Conclusions: LEEP excision for high-grade lesions and those with higher swede score should attempt in getting a minimum length of $0.5 \mathrm{~cm}$ and thickness of $0.35 \mathrm{~cm}$ for premenopausal and $0.75 \mathrm{~cm}$ for postmenopausal women. When doubtful, do not hesitate to perform multiple passes.

\section{Abstract \#232: Assessment of Sexual Function After LEEP In Precancerous Lesions of the Cervix-A Prospective Observational Study}

Amrita Gaurav, Dhriti Kapur, Kavita Khoiwal, Shivaani Arora, Jaya Chaturwedi

Department of Obstetrics and Gynaecology, AIIMS Rishikesh, Uttarakhand, India

Objective: To examine the effect of LEEP on overall sexual satisfaction and other specific aspects of sexual function in women with cervical dysplasia.

Methods: 61 sexually active women underwent LEEP for high-grade CIN II and CIN III (April, 2020-Sept, 2020) in the Department of Obstetrics and Gynaecology, AIIMS, Rishikesh and were interviewed regarding their sexual function before and after the LEEP procedure with TOOL structured sexual function questionnaire. Statistical analysis done using SPSS, version 11.5. Using paired t-test a $\mathrm{P}$ value of $<0.05$ considered statistically significant.

Result: The change in frequency of sexual intercourse was not statistically significant ( $\mathrm{p}$ value 0.960 ) whereas the pain/discomfort and postcoital bleeding episodes post-LEEP were statistically significant with a $\mathrm{p}$ value 0.016 and 0.001 , respectively.

Conclusions: Using LEEP to remove a part of the cervix may have negative effects on women's view of sexual function, resulting mainly from the effect of electrosurgery on nerve supply to the cervix and chronic inflammation associated with cervical tissue trauma. This study suggests that the deterioration in sexual function in women managed for cervical dysplasia is not a direct result of the treatment with LEEP. Having LEEP done along with other "nonsurgical" parts of cervical pre-cancer management is associated with small but statistically significant increase in overall sexual satisfaction.

\section{Abstract \#259: Prevalence and outcome of total abdominal hysterectomy performed for CIN}

Michael C. Ezeanochie, Emmanuel C. Anyanwu

Department of Obstetrics and Gynaecology, University of Benin Teaching Hospital, Benin City, Edo State, Nigeria

Objective: To review how common is the practice of Total Abdominal Hysterectomy (TAH) performed for Cervical intraepithelial Neoplasia (CIN).

Design: A retrospective, cross-sectional analytical study at the University of Benin Teaching Hospital (UBTH) in Nigeria from 2015 to 2019.

Method: It involved the review of the records of 145 patients who had total abdominal hysterectomy performed for benign gynaecological conditions. The primary outcome was the prevalence of CIN as the primary indication for total abdominal hysterectomy performed for benign Gynaecological conditions. Other outcomes were differences in the surgical outcome and complications. Statistical analysis used the Statistical Package for Social Sciences (SPSS) version 22. Categorical variables were compared using Chi-square or Fishers exact test while continuous variables were compared with the t test. Statistical significance was set at a p-value less than 0.05 .

Results: The mean age of the study population was $47.2 \pm 5.2$ years and $96.6 \%$ had at least 1 child. Majority of the women had Secondary School $(31.7 \%)$ or Tertiary $(54,5 \%)$ level of education. The commonest indications for TAH were uterine fibroids (51\%), endometrial 
Hyperplasia $(22.8 \%)$ and CIN (17.2\%). There was no significant difference in the surgical outcome between TAH performed for CIN compared to other benign Gynaecological conditions.

Conclusions: Hysterectomy for CIN is prevalent in this study. This may be considered an overtreatment and represents an unmet need for the appropriate treatment of premalignant disease of the Cervix.

\section{Abstract \#294: Title: Evaluation of factors in predicting outcome of loop electrosurgical excision procedure (LEEP)}

Amit Mandal, Anju Singh, Rajesh Kumari, Jayashree Natarajan, Sandeep Mathur, ShachiVashist, Sarita Kumari, Neerja Bhatla

Department of Obstetrics and Gynaecology, All India Institute of Medical Sciences, New Delhi, India

Objective: To compare colposcopy and cervical punch biopsy with loop electrosurgical excision (LEEP) results and estimate the potential impact of ablative procedures in high-grade cervical intraepithelial neoplasia (CIN2+).

Design: Retrospective study.

Methods: We analysed 40 cases of CIN2/3 who underwent LEEP with respect to age, parity, HPV load, and cytology and compared colposcopy and cervical biopsy findings with size of lesion and LEEP results.

Results: The mean age of women with CIN2 and CIN3was $40 \pm 9.1$ and $39 \pm 8.6$ years, respectively. Pre-LEEP diagnosis on cervical punch biopsy was CIN2 in $32.5 \%$ (13/40) and CIN3 in $67.5 \%$ (27/40). Post-LEEP, diagnosis was upgraded in $3 / 13$ (23.0\%) from CIN2 to CIN3, and in $6 / 27(22.0 \%)$ from CIN3 to invasive cancer. Thus final diagnosis was CIN2: 10; CIN3: 24; invasive cancer: 6 . All 6 invasive cancer cases had lesion $\geq 3$ quadrants. However, LEEP specimen of $12 / 24(50 \%)$ cases who had CIN3 on punch biopsy showed only chronic cervicitis, all of them had only 1-2 quadrant involvement. Cytology report was ASC-H in 4/10 (40\%) CIN2 cases, and HSIL in 11/24 (45.8\%) of CIN3 cases. One patient had bleeding complication after procedure which was managed conservatively. Nine $(37.5 \%)$ cases of post-LEEP CIN3 underwent hysterectomy for various reasons, 3 of them had positive margins post-re-LEEP. Two women had successful term pregnancies within one year of performing LEEP.

Conclusion: LEEP is an effective procedure for treatment of large CIN2 + lesions. However ablative procedure may be an option in lesions involving $\leq 2$ quadrants.

\section{Abstract \#297: Clinical evaluation of cold knife conization for diagnosis and treatment to cervical cancer patients}

Chao Zhao, Shiyan Wang, ShuoLiu, Chong Meng, Jianliu Wang, LihuiWei

Peking University People's Hospital, Xicheng District, Beijing, P.R. China

Objective: To evaluate the clinical value of conization in the diagnosis and treatment of cervical cancer patients.

Methods: 125 patients diagnosed with cervical cancer after cold knife conization in our hospital between 2008and 2016were reviewed. The pathological profiles of conization specimens and treatment options were analysed.

Results: Among them, there were 116 squamous cell carcinomas, 7 adenocarcinomas, 1 neuroendocrine carcinoma and 1 adenoid basal carcinoma. In 116 squamous cell carcinomas, there were stage Ia1 $(n=77)$, stage Ia2 $(n=5)$ and stage Ib1 $(n=34)$. In 7 adenocarcinomas, there were stage Ia1 $(n=3)$, stage Ia2 $(n=2)$ and stage Ib1 $(\mathrm{n}=2)$. Among 41 patients with stage Ia1 squamous cell carcinomas undergoing further surgery,14 (34.1\%) had residual diseases, including CIN II $(\mathrm{n}=9)$, CIN3 $(\mathrm{n}=4)$ and squamous cell carcinoma $(\mathrm{n}=1)$. No recurrence was found in 36 cases without further surgical intervention. All cases of stages $\mathrm{Ia} 2$ and $\mathrm{Ib} 1$ underwent repeat surgery.

Conclusion: Cold knife conization plays a very important role in the diagnosis and treatment of cervical cancer patients. Further treatment options should be selected individually and comprehensively based on such factors as patient's age, degrees of dysplasia, surgical margin status, fertility requirements and so on.

\section{Abstract \#330: A clinical study of correlation between colposcopy directed punch biopsy and LEEP in CIN}

Monika Singh, Rujuta Fuke, M.M. Kamal

Department of Obstetrics and Gynaecology, GMCH, Nagpur, India

Objectives: To correlate between colposcopy-directed punch biopsy and LEEP (loop electrosurgical excision procedure) in cervical intraepithelial neoplasia and also to determine the prevalence of cervical intraepithelial neoplasia in patients based on abnormal colposcopy findings.

Design: Prospective observational study.

Methods: All women attending Gynaecological Outpatient department at tertiary care centre who had given consent for study and met the inclusion and exclusion criteria of reproductive age group (22-55 years) screened for CIN ( cervical intraepithelial neoplasia) by VIA (visual inspection via acetic acid) and/or cervical cytology by liquid-based cytology. All the patients with positive VIA and abnormal cervical cytology findings will be subjected /screened by colposcopy examination. A sample size of 150 patients was taken for performing colposcopy.

Results: A total of 150 were subjected to colposcopy-directed biopsy, and only 39 subjects were reported to have CIN and rest of subjects were diagnosed as having cervicitis. LEEP was performed on these 39 subjects. The Kappa statistics were 0.4104 with agreement of $64.10 \%$ with $p$ value 0.0001 and Spearman's correlation was 0.5335 with $p$ value 0.0005 which was highly significant.

Conclusions: There is statistical correlation between colposcopy-directed biopsy and LEEP in patients of cervical intraepithelial neoplasia and LEEP is gold standard.

\section{Subtheme: Follow up}

\section{Abstract \#131: Outcome of CIN 2 Treatment over a 10-year period}

Rachana Dwivedi, Vicky Periera

Royal College of Obstetrics \& Gynaecology, United Kingdom

Objectives: A comparative study evaluating outcomes over a 10 -year period of three modalities of management of CIN2 including observation, cold coagulation or cervical excisions mainly assessing recurrence rate of pre-malignant lesion, time interval following treatment and associated risk factors. Additional parameters assessed were HPV persistence and correlation with outcome.

Methods: A retrospective study of 1000 women diagnosed with CIN2 on histology between 2010-2019 was done.

Results: Preliminary data analysis shows mean age of 29 years with index smears $<$ low grade in $73 \%$, high grade- $25 \%$, other indication$2 \%$; size of lesion $-1 / 2$ quadrants in $25 \% ; 3 / 4$ quadrants in $9 \%$. Cold coagulation was undertaken in $27 \%$, LLETZ in $66 \%$ and conservative 
management in $7 \%$ cases. In conservative management group, followup smear at 6 months was negative in $33 \%$; $<=$ low grade in $33 \%$. Subsequent follow-up smear showed high grade in $33 \%$ with time interval from treatment ranging from 6 months to 4 years.

\begin{tabular}{lllc}
\hline & $\begin{array}{l}\text { Follow-up } \\
(\%)\end{array}$ & $\begin{array}{l}\text { Negative HR HPV } \\
(\%)\end{array}$ & Time Interval \\
\hline $\begin{array}{l}\text { Cold } \\
\text { coagulation }\end{array}$ & 66 & 33 & $\begin{array}{c}6 \text { months- } \\
8 \text { years }\end{array}$ \\
$\begin{array}{c}\text { LLETZ } \\
\text { 6 months- } \\
4 \text { years }\end{array}$ \\
\hline
\end{tabular}

Conclusions: Majority of patients underwent excisional treatment as opposed to ablation and conservative management. Although recurrence rate with excisional treatment was comparatively lower than cold coagulation or conservative management, risk of relapse of premalignant disease may occur after time interval up to 8 years, hence vigilance regarding previous abnormal smear and/or treatment is essential. Excisional treatment especially depth $>=15 \mathrm{~mm}$ is associated with significant risk of preterm birth contributing to obstetric morbidity burden. Patient should have informed choice and offer conservative/surgical options based on current evidence. P16negative/index smear/nulliparity should trigger conservative option as first choice.

\section{Abstract \#322: High-Risk Human Papillomavirus DNA Testing Predicts Residual/Recurrent Cervical Intraepithelial Neoplasia After Treatment By LEEP}

Lutfa begum Lipi, Iffat Ara, Khodeja Begum

Dhaka Medical College, Dhaka, Bangladesh

Background and Objective: Therefore, the presence of certain highrisk HPV genotypes indicate a significant risk factor for the development of cervical cancer. This study was undertaken to evaluate the value of high-risk HPV testing in the follow-up period of high-grade cervical intraepithelial neoplasia (CIN2/3) treated by Loop Electrosurgical Excision Procedure (LEEP) as a predictor of recurrent/ residual cervical intraepithelial neoplasia.

Methods: Retrospectively 46 participants were analysed in a single tertiary hospital. Colposcopic and punch biopsy-proven CIN-2/3 were treated by LEEP. Post-LEEP follow-up was performed by colposcopy and HPV DNA by Hybrid Capture II test. Demographic variants were also analysed. The definition of persistent/recurrent disease was biopsy-proven CIN.

Results: Among 46 participants, mean age was $32.43 \pm 4.45$ (25 to 40 years), $\mathrm{CIN}-2$ were $42(91.3 \%) \& \mathrm{C} / \mathrm{N}-3$ were $4(8.7 \%)$. Before LEEP HPV HC II test was not possible due to do as low-resource setting and cost but during follow-up at 6 months HPV DNA test by Hybrid capture II was done in 46 patients. Colposcopy punch biopsy was taken. Five patients $(10.87 \%)$ had residual/recurrent cervical intraepithelial neoplasia with HPV DNA + ve (patient RLU/known positive RLU ratio $>1.2$ ). Though margin status was a significant predictor for persistence/recurrent CIN, early HPV DNA testing at 6 months after LEEP predict all case of residual/recurrent disease. Sensitivity and negative prediction value of HPV HC II test for residual/recurrent disease were $100 \%$ at 6 months.

Conclusion: Persistence or clearance of high-risk human papillomavirus is an early valid prognostic marker of failure or cure after treatment for CIN 2/3 and is more accurate than cytology and section margin. This early HPV testing implies an important role to reduce amount of post-treatment follow-up.

\section{Cervical cancer}

\section{Early cervical cancer}

Paper number \#316: Anenga Ushakuma Michael, Dr HembahHilekaan Samuel K

Benue State University Teaching Hospital, Makurdi- Nigeria, Nigeria A 7-Year Review of Cervical Cancer in Benue State University Teaching Hospital, Makurdi

\begin{abstract}
Background: Cervical cancer is the fourth most common cancer in women worldwide and the second most common female cancer in women. In Nigeria, it is the second most common cancer and most common cancer among the female population in the country. Human papillomavirus (HPV) infection of the cervix is implicated in its aetiology. Cervical cancer is preventable through early diagnosis using screening tests and vaccination.
\end{abstract}

Objective: To evaluate and establish the socio-demographic characteristics, clinical factors, prevalence and pattern of cervical cancer patients presenting at Benue State University Teaching Hospital (BSUTH) Makurdi within the study period.

Materials and Methods: A seven-year retrospective study of cases of histologically diagnosed cervical cancer at BSUTH, Makurdi from 2012 to 2018 was undertaken.

Results: The prevalence of cervical cancer among gynecological malignancy was $57.5 \%$. The mean age was 49.8 years \pm 14.6 , with peak incidence occurring at age range of 35-44 years. The highest incidence occurs among those with parity 5 and above (53.2\%). Squamous cell carcinoma was the most common reported histopathology $(97.4 \%)$. Early stage disease (stage I - IIa) comprised $18.2 \%$, while $81.8 \%$ presented in advanced stage (stage IIb - IV). Most cases of histologically confirmed cancer were referred to centres with facilities for radiotherapy. However, early stage diseases were treated at BSUTH.

Conclusion: There is a high prevalence of cervical cancer in this study. So, there is need for organized cervical cancer screening for early detection and treatment of the disease.

Keywords: Cervical cancer, Screening, Gynaecological malignancies

\section{Abstract \#63: Prognostic significance of histological types in early} stage cervical cancer post-radical hysterectomy

Anjana J S, Rema P, Suchetha S, Sivaranjith J, Dhanya D

Regional Cancer Centre, Thiruvananthapuram, Kerala, India

Objectives: To compare the recurrence rate and survival outcome, between different histological types, in patients who underwent radical hysterectomy for early cervical cancer.

Methods: A retrospective study comparing the outcome of patients with squamous cell carcinoma (SCC) and adenocarcinoma (AC) who underwent radical hysterectomy for early stage cervical cancer at RCC Trivandrum from 1 January 2007 to 31 December 2018. Clinicopathological features, treatment details, recurrence and survival details were collected from medical records. Kaplan-Meier survival curves were generated to assess the disease-free and overall survival. Results: A total of 130 patients, 89 with SCC and 41 with AC, underwent radical hysterectomy during this time period. The patient 
profile and preoperative FIGO stage, were comparable between the two groups. The number of patients having tumors size greater than $4 \mathrm{~cm}$ was higher in case of AC compared to SCC (19.5\% vs $2.2 \%$, p-value 0.002). Significantly higher percentage of patients with AC received adjuvant therapy compared to patients with SCC (75.6\% vs $53.9 \%$, p-value 0.021 ). The median follow-up period was 80 months. The 3-year disease-free survival was $94.9 \%$ and $94.2 \%$ (p-value 0.988 ) for AC and SCC, respectively, and 3-year overall survival was $97 \%$ and $100 \%$ (p-value 0.194 ) showing no statistically significant difference in the survival outcome between the two groups.

Conclusions: Patients with adenocarcinoma tends to have larger tumor size, and are more likely to receive adjuvant therapy. The histological subtype (AC v/s SCC) does not have an impact on the survival outcome of patients with early cervical cancer.

\section{Abstract \#97: SLNB (Sentinel Lymph Node Biopsy) in Carcinoma Cervix}

Sasikumari M, S.P Somashekhar, Ahuja Vijay, K.R Ashwin, Kumar Rohit

Manipal Hospital, Bengaluru, India

Objectives: To evaluate the feasibility and usefulness of SLNB technique in our series of 20 consecutive women with early stage cervical cancer (FIGO Stage 1B1, lb2). All were Open Surgeries.

Methods: A total of 20 consecutive biopsy-proven cases of Carcinoma cervix with FIGO Stage IB1 \& lb2 were included. Patients who were Stage-II \& above, those with previous pelvic radiotherapy and retroperitoneal surgery or with previous Patients who had undergone chemotherapy previously were excluded.

Results: Sentinel node was identified in $100 \%$ (n-20) cases, and $90 \%$ of time it was the Pelvic node, $10 \%$ of time SLN was present at the Aortic bifurcation. Mean of 2.7 sentinel nodes were identified, Mean size of positive sentinel node $=0.8 \mathrm{~mm}$. In $33.3 \%(\mathrm{n}=2)$ cases the Sentinel node was the only node positive. Only pelvic SLNs was seen in $80 \%(\mathrm{~N}=16)$ cases, in $12 \%$ at obturator area, in $34 \%$ at the origin of the uterine artery-lliac, $15 \%$ at the division of the common iliac artery, in $14 \%$ bilateral nodes and in $5 \%$ parametrial node.

Conclusion: SLN biopsy is a feasible, safe and useful technique in early stages of carcinoma cervix. Sentinel lymph node identification, may improve the ability to detect lymph node metastases in patients who have cervical cancer. SLNB in early stages of cervical cancers is a promising surgical technique to confirm nodal status and minimise post-operative morbidity avoiding unnecessary lymphadenectomy in presence of negative SLN. Patients detected to have positive SLNB, can just receive concurrent chemoradiotherapy as definitive treatment thus avoiding the morbidity of surgery and chemoradiation which will be required as part of definitive treatment.

\section{Abstract \#119: Synoptic Operative Report in Cervical Cancer Surgeries: Experience from Single Oncology Center}

Nilanchali Singh, Michelle Chanco, Vivian Wen, Neha Mishra, Prafull Ghatage

Tom Baker Cancer Centre, Canada

Objective: Recording of patients' operative data is important for patients' management, training as well as for research purpose. It leads to standardized and comprehensive reporting of surgical events. It also mandates that the surgical residents have a better understanding of all the facets of procedure. This study aims at analyzing the reporting by this database.

Design: It is a retrospective observational study.
Methods: Alberta Cancer Registry was contacted for obtaining data from the Synoptic Operative Reports of all the patients operated for cervical cancer between December 2009, when Synoptic reporting of cervical cancer was started to February 2020.

Results: The data comprises 463 patients operated for Stage 1 and 2 cervical cancers and 10 patients operated for advanced and recurrent cervical cancer. 101 patients were operated for carcinoma in situ. Adenocarcinoma was the most common histology. Laparotomy was done in 308 patients whereas others had some form of laparoscopic procedure. The details of surgery from beginning of incision to closure were detailed. The template of cervical cancer comprised of 356 questions. There were separate templates for advanced and early stage cancer. However, with this meticulous detail, only $8 \mathrm{~min}$ (average) were taken by each user to complete the template.

Conclusions: Synoptic reports help to maintain the quality and consistency of operation notes by ensuring that all reports contain standardized fields.

\section{Abstract \#168: Redistribution of Early Cervical Cancer Patients from FIGO 2009 to FIGO 2018}

AnilaTresa Alukal, Rema P, Suchetha S, Dhanya Dinesh, Sivaranjith J, Aleyamma Mathew

Regional Cancer Centre, Thiruvananthapuram, Kerala, India

Background: A major change in the FIGO 2018 staging is the incorporation of imaging and pathological findings when compared to FIGO 2009 which had only clinical examination. Another major change is introduction of IB3 for bulky tumours and III C for nodal disease.

Objective: The aim of this study was to evaluate the upstaging of early cervical cancer patients when staged according to the FIGO 2018.

Design: Retrospective cohort study.

Methods: The medical records of 131 patients who underwent radical hysterectomy for early invasive cervical cancer in our department from January 2008 to December 2018 were reviewed. The patients were initially distributed according to FIGO 2009. The same group of patients were then redistributed according to FIGO 2018 based on final histopathology.

Results: There were $82.4 \%$ patients in IB1, $8.4 \%$ in IB2, $9.2 \%$ in IIA1when staged according to FIGO 2009. Only $73.3 \%$ patients had a preoperative imaging. On redistributing according to FIGO 2018, there were $35.9 \%$ in IB1, 38.9\% in IB2, $6.9 \%$ in IB3, 5.3\% in IIA1, $1.5 \%$ in II B, $11.5 \%$ in IIIC. Vaginal involvement was seen in $12.2 \%$, parametrial involvement in $1.5 \%$, nodal involvement in $11.5 \% .40 .5 \%$ of the patients received no adjuvant treatment. $41.2 \%$ patients received adjuvant radiation, and $18.3 \%$ patients received adjuvant concurrent chemoradiation with weekly cisplatin.

Conclusion: FIGO 2018 staging aids better in describing the extent of disease as well as treatment planning when compared to FIGO 2009.

\section{Abstract \#172: Adjuvant Treatment After Radical Hysterectomy for Early Cervical Cancer}

AnilaTresa Alukal, Rema P, Suchetha S, Dhanya Dinesh, Sivaranjith J, Aleyamma Mathew Aswinkumar, Thara Somnath, Ammu JV

Regional Cancer Centre, Thiruvananthapuram, Kerala, India

Objectives: The recommendation of adjuvant treatment following radical hysterectomy in early stage cervical cancer is usually tailored according to International Federation of Gynaecology and Obstetrics (FIGO) stage and risk factors. The aim of the study was to assess the 
outcome of early cervical cancer patients who underwent radical hysterectomy followed by adjuvant treatment.

Design: Retrospective cohort study.

Methods: The medical records of 131 patients who underwent radical hysterectomy for early stage cervical cancer FIGO 2018 Stage I to IIA1 were assessed for clinical and histopathologic features. The patients were grouped into no adjuvant treatment, adjuvant radiation or adjuvant chemoradiation with weekly cisplatin. The adjuvant treatment received was based on the presence of high risk factors like nodal, parametrial or margin involvement and intermediate risk factors like lymph vascular involvement, deep stromal involvement or size $>4 \mathrm{~cm}$.

Results: $40.5 \%$ of the patients received no adjuvant treatment. $41.2 \%$ patients received adjuvant radiation, $18.3 \%$ patients received adjuvant concurrent chemoradiation. The overall survival in all three groups was $100 \%$. The disease-free survival in no adjuvant treatment group was $92 \%$, in adjuvant radiation group was $96.2 \%$ and adjuvant chemoradiation group was $100 \%$. Recurrence was noted in 5 patients who had no adjuvant treatment, 4 who had adjuvant radiation and one patient who had adjuvant concurrent chemoradiation.

Conclusion: Adjuvant concurrent chemoradiation should be considered in early stage cervical cancers following radical hysterectomy with high-risk features as it has better survival outcome.

\section{Abstract \#174: Survival Outcome and Prognostic Factors of Open Radical Hysterectomy for Early Cervical Cancer}

Anila Tresa Alukal, Rema P, Suchetha S, Dhanya Dinesh, Sivaranjith J, Aleyamma Mathew, Aswinkumar, Thara Somnath Ammu JV

Regional Cancer Centre, Thiruvananthapuram, Kerala, India

Objective: Early cervical cancer is classically treated by open radical hysterectomy and bilateral pelvic lymph node dissection. To assess the survival outcome and prognostic factors of early cervical cancer patients treated by Open Radical Hysterectomy.

Design: Retrospective cohort study.

Methods: The medical records of patients who underwent radical hysterectomy for early cervical cancer from January 2008-December 2018 were analysed for demographic, clinical and histopathologic features. Kaplan Meir survival curves were generated to assess the disease-free survival and overall survival.

Results: The mean age at presentation was 51.2 yrs. The most common presenting complaint was irregular bleeding seen in $58.8 \%$ discharge per vagina in $33.3 \%$. Screening detected cancer in $10.7 \%$. The most common histology was squamous cell carcinoma keratinising type seen in $45.8 \%$. The most common stage for which surgery was done was IB2. On final histopathology vaginal involvement was seen in $12.2 \%$, parametrial involvement in $1.5 \%$, nodal involvement in $11.5 \% .40 .5 \%$ of the patients received no adjuvant treatment. $41.2 \%$ patients received adjuvant radiation, $18.3 \%$ patients received adjuvant concurrent chemoradiation with weekly cisplatin. Recurrence was seen in $7.6 \%$. Death due to disease was $0.8 \%, 8.4 \%$ was lost to follow-up. The overall survival was $100 \%$ and the disease-free survival was $95.3 \%$ for a median follow-up of 90 months.

Conclusion: Open radical hysterectomy and bilateral pelvic node dissection have an excellent survival outcome and should remain as the standard of care for early cervical cancers.

\section{Abstract \#175: Is Imaging Mandatory for Staging of Early} Cervical Cancer

Anila Tresa Alukal, Rema P, Suchetha S, Dhanya Dinesh, Sivaranjith J, Aleyamma Mathew, Aswinkumar, Thara Somnath, Jagathnath Krishna KM

Regional Cancer Centre, Thiruvananthapuram, Kerala, India

Objectives: FIGO staging for cervical cancer was revised in 2018 by including imaging and pathological findings, to assign the stage and planning treatment. Now it is a routine practice to do MRI for early cervical cancer. The aim of our study was to assess the sensitivity, specificity and diagnostic accuracy of MRI and clinical examination as compared to the final histopathology.

Design: Retrospective cohort study.

Methods: The medical records of 88 patients who underwent radical hysterectomy for early invasive cervical cancer in our department from January 2008 to December 2018 were reviewed. The clinical and radiological details including stage, size of the tumor, vaginal involvement, parametrial involvement were noted. This was compared with the final histopathology.

Results: For tumors less than $2 \mathrm{~cm}$, clinical examination \& MRI had a comparable diagnostic accuracy of $77.8 \& 73.1$. For detecting parametrial involvement, clinical examination had $98.8 \%$ specificity, with NPV (negative predictive value) of 97.7, a diagnostic accuracy of 96.6 similar to MRI that was $96.5 \%$ specificity, NPV $97.64 \%$ and a diagnostic accuracy of 94.3 .

For detecting vaginal involvement, both clinical examination and MRI had a NPV of $86.75 \%$ and a diagnostic accuracy of 84.1 . For lymph node detection, MRI had $100 \%$ specificity and a NPV of $94.19 \%$.

Conclusion: MRI and clinical examination had comparable sensitivity, specificity and diagnostic accuracy for determining tumor size, vaginal and parametrial involvement. Hence in developing countries where routine radiological imaging may not be cost-effective for early cervical cancers, it may be prudent to stick to clinical examination for staging and rely on pathology for nodal involvement.

\section{Abstract \#193: Prognostic Significance Of Histological Types In Early Cervical Cancer Post-Radical Hysterectomy}

Anjana J S, Rema P, Suchetha S, Siva Ranjith J, Dhanya Dinesh, Jagathnath Krishna

Regional Cancer Centre, Medical college Kumarapuram road Medical college campus Chalakkuzhi Thiruvananthapuram, Kerala, India

Objectives: To compare the histological features, recurrence rate and survival, between different histological types, in patients who underwent radical hysterectomy (RH) for early cervical cancer.

Design: Retrospective study.

Methods: This study compared the outcome of patients with squamous cell carcinoma (SCC) and adenocarcinoma cervix (AC) who underwent RH at our institute from 1January 2007 to 31 December 2018. Patient details were collected from medical records. KaplanMeir survival curves were generated to assess disease-free survival (DFS) and overall survival (OS).

Results: A total of 136 patients with early cervical cancer underwent RH during this time period. 95 patients $(69.8 \%)$ had SCC and 41 patients $(30.1 \%)$ had AC. The baseline patient profile, preoperative FIGO stage were comparable between the two groups. The number of patients having tumors size greater than $4 \mathrm{~cm}$ were higher in case of AC compared to SCC (19.5\% vs $2.2 \%$, p value 0.002$)$. The rate of parametrial involvement and nodal metastasis were comparable. Significantly higher percentage of patients with AC received adjuvant 
therapy compared to SCC (75.6\% vs $53.9 \%$, p value 0.021$)$. The median follow-up period was 74 months. The 3 year DFS was $92.2 \%$ and $95.5 \%$ ( $\mathrm{p}$ value 0.504 ) for AC and SCC, respectively, and the 3 year OS was $97 \%$ and $100 \%$ (p value 0.194 ).

Conclusions: Patients with adenocarcinoma tend to have larger tumor, and are more likely to receive adjuvant therapy. The histological subtype does not have an impact on survival in patients with early cervical cancer undergoing $\mathrm{RH}$.

\section{Abstract \#194: Clinicopathological Profile of Cervical Carcinoma: An Experience of Secondary Care Rural Hospital in Andhra Pradesh, India}

\section{P. Jerusha Evangelin, M. Jyothi Suchitra, N. Sundari}

Department of Obstetrics and Gynaecology, Rural development trust hospital, Bathalapalli, Andhra Pradesh, India

Objectives: Cervical cancer is one among the preventable cancers but still remains the most common and dreaded diseases of women today. In spite of best screening procedures, lack of awareness, social stigma and fear are the main barriers to cervical cancer screening. To assess the histopathology, clinical profile of patients presenting with cervical carcinoma and to identify factors associated with timing of presentation and prognosis in secondary care rural hospital in Andhra Pradesh, India.

Methods: This is a retrospective analysis from January 2018 to December 2020 at Rural development trust hospital, Bathalapalli. A pretexted data extraction sheet aimed at collecting information from inpatient records was used as study instrument. Collected data is analyzed using SPSS software version 20.

Results: A total of 261 patients were included in the study. Mean age of diagnosis of cervical cancer was found to be 4th and 5th decade $(40 \%)$. History of bleeding per vaginum $(80 \%)$ is the most common clinical presentation. Stage III cases constituted in majority (70\%), and also had poor prognosis. Squamous cell carcinoma (89\%) was the most common histological variant followed by adenocarcinoma $(4.6 \%)$. Wertheim's hysterectomy was done in $10 \%$ of cases in our hospital under the guidance of oncologist.

Conclusions: In spite of being one of the most preventable cancer with best screening procedures and long premalignant phase, most women presented in later stages of cervical cancer. Active intervention, high index of suspicion, health education and dedicated screening programs are needed to achieve the desired result in cervical cancer prevention.

\section{Abstract \#248: Methylene blue dye for sentinel lymph node mapping in early cervical cancer}

Nisha Singh, Shuchi Agrawal

Department of Obstetrics and Gynecology, King George medical university, Lucknow, India

Objective: To validate the use of methylene blue dye for intraoperative sentinel lymph node mapping in cases of early cervical cancers. Design: Cross-sectional cohort study.

Methods: The study included 30 cases of early stage cervical cancer where sentinel lymph node mappings was done. Four milliters of methylene blue was injected submucosally in the cervix. Bilateral pelvic lymph nodes were examined visually for any blue staining. Both stained and unstained lymph nodes were sent for histopathological examination separately from each side. HPE results were recorded to compare presence of metastasis in stained and unstained lymph nodes.
Result: Out of 30 SLN mappings, staining of sentinel LN was seen in 25 , suggesting a detection rate of $83 \%$. Average number of lymph nodes removed in each case was 10.3. The sentinel LN was most commonly present among right external iliac nodes. Bilateral sentinel lymph nodes were seen in 6 cases. All stained and unstained nodes among the 25 SLN detections did not show any histological evidence of metastasis suggesting a NPV of $100 \%$.

Conclusion: Methylene blue is an effective, feasible and safe tracer to detect sentinel lymph nodes in early stage cervical cancer.

\section{Abstract \#258: Comparision of Pre- and Post-operative Stage and Need for Adjuvant chemoradiotherapy in Cervical Cancer as Per FIGO 2018}

Sharma Divya, Singh Nisha, Srivastav Kriti, Jaiswal Riddhi, Agarwal Shuchi

Department of Obstetrics and Gynecology, King George medical university, Lucknow, India

Objective: To compare preoperative (clinic-radiological) and postoperative (surgico-pathological) staging in early-stage cases of cervical cancer on the basis of advancement made in FIGO 2018 staging and to analyse the reasons for need of adjuvant CTRT.

Design and Method: An observational hospital-based study was conducted in which 92 cases with early-stage cervical cancer were recorded over a period of 5 years i.e. from 2016-2019 (retrospective87 cases) and 2020-21 (prospective 5 cases). Cases were re-staged as per FIGO 2018 staging, on basis of findings in clinical examination, preoperative imaging, per-op and histopathology (HPE) report, but HPE was used to determine the final stage.

The data obtained was used to assess the number of cases who underwent an upstage on basis of FIGO 2018, the primary mode of treatment given and to analyze the reasons for need for adjuvant CTRT.

Results: In all the 92 cases enrolled on comparing the pre- and postop staging, $40.8 \%$ of cases were upstaged on basis of HPE report. Maximum number of upstage were made on the basis of positive lymph nodes found either on pre-op or HPE report $(45.4 \%)$, followed by depth of invasion (31.8\%) and least by tumor size (22.7\%). Of all the operated cases $36.8 \%$ cases underwent adjuvant CTRT, followed by primary surgery; most common reason being positive lymph nodes (47.6\%), followed by parametrial invasion and deep stromal invasion $(33.3 \%)$ and least by lymphovascular space invasion (LVSI) $(19.4 \%)$. Conclusion: Nearly more than one-third of all the operated cases underwent an upstage as per FIGO 2018 staging system. The new staging system being surgicopathological helps in better determination of primary treatment and also the need for adjuvant CTRT for early stage cervical cancer cases.

\section{Abstract \#284: Predictive factors of lymph node positivity in cervical cancer \\ Dhanya Dinesh, Rema P, Suchetha S, Siva Ranjith J \\ Regional cancer centre, Thiruvananthapuram, India}

Objectives: To evaluate the factors predicting lymph node positivity in cervical cancer.

Methods: A retrospective study of all patients who underwent radical hysterectomy for presumed early cervical cancer in Regional cancer centre, Thiruvananthapuram between January 2007 and December 2017 was carried out. Clinical, demographic, surgical and histopathologic details were obtained from the hospital database. 
Results: 134 patients underwent surgery for presumed early cervical cancer during the study period. Of these 18 (13.4\%) patients had positive nodes in final histopathology. $12 \%$ of premenopausal patients and $14.4 \%$ of postmenopausal patients were node positive. Node positivity was seen in $13.04 \%$ of patients with squamous cell carcinoma and $10.8 \%$ of patients with adenocarcinoma cervix. Among patients with tumour size $<2 \mathrm{cms}, 3.38 \%$ had positive nodes, compared to $21.33 \%$ in patients with tumour size $>2 \mathrm{cms}$. Among patients with depth of stromal invasion $>2 / 3$ of cervix, $43.2 \%$ had positive nodes, compared to $2.4 \%$ in those with stromal invasion $<2$ / 3 . Node positivity was seen in $55.5 \%$ of patients with lymph vascular space invasion (LVSI) compared to $12.2 \%$ of patients without LVSI. Conclusion: Among the variables assessed, tumour size more than 2 cms, depth of stromal invasion more than $2 / 3$ and the presence of lymph vascular space invasion were found to be significant predictors for lymph node positivity in cervical cancer.

\section{Advanced Cervical Cancer}

\section{Abstract \#140: Challenges in management of recurrent cervical cancer}

Leela Digumarti, Priyanka Vemanamandi, Vidya Viswanath, Mohanarao Dasu, Raghunadharao Digumarti

Homi Bhabha Cancer Hospital \& Research Centre, Vishakhapatnam, India

Objectives: To analyze the morbidity and mortality of recurrent cervical cancer and its management.

Methods: A retrospective analysis from 1 January 2015 to 31 December 2015. All women with recurrence are included.

Results: There were a total of 435 women with carcinoma cervix during the study period. Out of these, 65 had recurrence at presentation. All had received primary treatment elsewhere.

Age: 30 to 80 years, $46 \%$ in the age group of 40 to 59 and $29 \%$ in the range of 60 to 69 years.

Stage: $2 \mathrm{~b}$ are $41 \%$ and $3 \mathrm{~b}$ are $33 \%$. Chemoradiotherapy (CT + RT) was the primary treatment given in $75 \%$ of women. The diseasefree interval varied from a few months to as high as 25 years.

Local recurrence was seen in $41 \%$, distant metastasis in lungs and liver in $23 \%$, bones in $5 \%$ and in para-aortic lymph nodes in $16 \%$. Palliative chemotherapy was given to $41 \%$ and radiotherapy for bone mets to $7 \%$. All 65 needed pain management with more than $50 \%$ receiving morphine. Fistulas were seen in $12 \%$. A rectovaginal fistula needed a diversion stoma. Another underwent bilateral groin node dissection and an anterior exenteration with a neo bladder. To date, only 2 women are disease-free and on regular follow-up.

Conclusions: Recurrence in cervical cancer is mainly regional failure. Pain is a major symptom, majority needing morphine. Intractable symptoms include fistulas, occasionally needing diversions. Palliative chemotherapy has poor outcome but abundant morbidity. Survival is very poor.

\section{Abstract \#182: Brain Metastasis in Carcinoma Cervix-Single Institution Study}

Rupinder Sekhon, Amita Naithani, Priyanka Makkar, Pratima R

Rajiv Gandhi Cancer Institute \& Research Centre, Rohini, Delhi, India

Objectives: The occurrence of brain and central nervous system (CNS) metastasis from gynaecological malignancy in rare, occurring in only $1 \%$ of all female genital tract malignancy and even rarer is the metastasis to brain from primary carcinoma cervix. There is minimal data on these "neuro-phobic tumours.

Methods: All patients of Carcinoma Cervix treated at Rajiv Gandhi Cancer Institute and Research Centre (RGCIRC) between January 2007 till January 2020 were included. A retrospective analysis of incidence, pattern of recurrence and survival rates was done.

Results: A total of 3240 patients of carcinoma cervix were treated at RGCIRC during the study period.

The median age of CNS recurrence in carcinoma cervix was 55 years. A total of 4 CNS, 3 vertebral and 2 paravertebral soft tissue deposits were reported. The commonest site of recurrence in CNS was cerebral hemisphere, a total of 3 cases $(0.12 \%)$ followed by cerebellum in 1 case. Median time of CNS recurrence from the diagnosis of primary disease was 22.75 months, with the longest time duration of recurrence being 41 months. Overall survival post-CNS recurrence was approximately 7 months with all patients succumbing to disease during the further follow-up. Longest survival being 11 months 13 days and shortest being 1 month 20 days post-brain metastasis.

Conclusions: Rare metastatic recurrence of an otherwise common gynaecological cancer has been reported in this study. The recurrence is not related to the tumour histology and has a poor prognosis uniformly. Abstract \#183: Immune Status in Predicting Response to Chemo-
Radiation in Cervical Cancer Patients: Prognostic clue

Swati Garg, Usha Sekhawat, Rajat Vohra

Mahatma Gandhi Medical College and Hospital, Jaipur, India

Objectives: To evaluate the prognostic role of pretreatmentCD4+, $\mathrm{CD} 8+$ in predicting response to definite chemo radiotherapy in advanced cervical cancer.

Design: A hospital-based prospective 1-year follow-up study.

Methods: This study was conducted in 74 patients of advanced cervical cancer. Pre-treatment tumour infiltration with $\mathrm{CD} 4+$ and $\mathrm{CD} 8+$ in cervical cancer tissue and peripheral blood was noted and quantitatively assessed in patients with remission or persistent disease after one year of follow-up.

Results: There was statistically significant association of tumour volume with the remission or persistence of disease. In peripheral blood, mean $\mathrm{CD} 4+$ score and $\mathrm{CD} 4+/ \mathrm{CD} 8+$ ratio was significantly higher, while mean CD8 + score is significantly lower in patients with remission. Similar results were seen in tumour tissue as well. On receiver operating curve analysis, the cut-off value of $\mathrm{CD} 4+, \mathrm{CD} 8+$ and $\mathrm{CD} 4+/ \mathrm{CD} 8+$ ratio in predicting remission or persistent disease in peripheral blood was $20.09,18.51$ and 0.41 while in tumour tissue was $19.71,20.99$ and 0.20 , respectively. Conclusions: The patients with tumour volume $<100 \mathrm{~cm}^{2}$ have much higher chances of remission. The patients with higher CD4 + and CD4 + / CD8 + ratio, both in peripheral blood as well as tumor tissue, have higher chances of remission. The cut-off value of $\mathrm{CD} 4+, \mathrm{CD} 8+$ and $\mathrm{CD} 4+/ \mathrm{CD} 8+$ ratio in predicting remission or persistent disease in peripheral blood was 20.09, 18.51 and 0.41 while in tumor tissue was 19.71, 20.99 and 0.20 , respectively.

\section{Glandular Disease}

Abstract \#91: Outcomes of referrals with query glandular neoplasia on cytology-18 year review in a UK University teaching hospital

Deirdre Lyons, Selina Chiu, Corrina Wright, Thomas Lynch

Imperial College Healthcare NHS Trust, United Kingdom 
Objectives: Review all referrals with report suggestive of glandular neoplasia with regards to outcomes and management over an 18-year period. Assessment of reporting adherence and outcomes of colposcopy assessment and management. Abnormal cervical cytology reported as glandular neoplasia-uncommon, accounting for $0.05-0.1 \%$ of UK samples and are associated with high incidence of pre-invasive and invasive disease-20-28\% and $40-43 \%$, respectively. NHSCSP Colposcopy guidelines suggest all reports indicative of query glandular neoplasia should have a written descriptive report indicating likely source of abnormal glandular cells. Reliable diagnosis requires cylindrical-shaped excisional cervical biopsy in order to ascertain and treat pathology. Women with suspected CGIN or early invasive adenocarcinoma, the extent of the cervical excision can be individualised depending on age and preferences on fertility conservation.

Methods: All reports suggestive of query glandular neoplasia were extracted from Cytopathology database. Colposcopy database was interrogated to review clinical colposcopy assessment, management and outcomes of these referrals.

Results: Total colposcopy referrals over this time period -24.000 .

Total referrals with ?glandular neoplasia - $228(0.89 \%)$.

Review of outcomes in women less than 36 years old versus over 36 years old.

HG Pre-invasive disease-52.6\% ( $\mathrm{n}=120)$; Invasive disease$21 \% \quad(\mathrm{n}=48) ; \quad$ LGCIN/ benign-26.4\%; Non-cervical-8.3\% $(\mathrm{n}=19)$. Overall, pre-invasive/ invasive disease in this population$73.6 \%$

Patients $<36$ years old only had cervical disease.

Conclusions: Results in keeping with literature, identifying a high incidence of pre-invasive and invasive disease in referrals suggestive of glandular neoplasia.

\section{Abstract \#130: CGIN-Treatment and Outcomes-A 5 year London review}

Deirdre Lyons, Ola Folayan, Dan Zamblera, Jane Rains, Joe LLahi, Wendy Smith

Imperial College Healthcare NHS Trust, United Kingdom

Objectives: Assessment and Management of High-Grade Cervical Glandular Intraepithelial Neoplasia (HGCGIN) across London Units - 5 year review. Publications on management of HGCGIN demonstrate $19.4 \%$ developed recurrent in situ disease and $6 \%$ diagnosed with invasive disease, whereas with negative margins, risk of subsequent invasive disease was $0.35 \%$ and the risk of recurrent CGIN was $2.6 \%$. Assessment from London Units looked at outcomes in terms of age demographic, contraception, cytology at referral, incidence per year, number of excisions to clearance, subsequent cytology and HPV testing outcomes and number of invasive carcinomas diagnosed.

Methods: Query for CGIN outcome published to all London Units via Cyres. Time period for study-2015-2019 inclusive (5 years). Anonymised excel spreadsheet sent to lead author for collation and assessment from Units. Overall London data from National statistics publications.

Results: A total of 21 out of 26 London Units provided data for analysis. Attended referrals to London Units over time period was 189,157 . Outcomes with HGCGIN are $812 *$ over 5 year time period. For 21 London Units-651 patients had a diagnosis of HGCGIN. Most were referred with cytology suggestive of query glandular neoplasia-357 (55\%), Age demographic varied between the Units, most women were in 30-40 year-old age group. Excision was mainly LETZ. Most Units adhered to the guidelines of $10 \mathrm{~mm}$ depth for under 36 there were more second excisions for over 36 years old.
Conclusions: HGCGIN is uncommon and adherence to guidelines in terms of management should be regularly reviewed.

\section{Theme 6. Public health}

\section{Sharing the Experience in Mass Screening Program}

Abstract \#104: Comparison of screening practices for noncommunicable diseases with cervical cancer screening in a multidisciplinary teaching hospital in Delhi

Jasmine Chawla Sharma, Tanu Anand

Hindu Rao Hospital, Delhi, India

Objectives: This study was undertaken to assess the knowledge, attitude and practice (KAP) amongst female health workers in a tertiary hospital of Delhi regarding screening for noncommunicable diseases and comparing this with cervical cancer screening to assess barriers to acceptance of the pap test.

Materials and Methods: A cross-sectional, descriptive study was conducted with semi-structured, self-administered questionnaire among female health workers. The study subjects were interviewed for KAP regarding risk factors for cancer cervix, Pap test and HPV vaccination for protection against carcinoma cervix. This was compared with the screening practice for hypertension and diabetes. Descriptive statistics were used to characterize the study participants. For association, the Chi-square test was applied and $\mathrm{P}$ value $<0.05$ was considered significant.

Results: A large no of female health workers had good knowledge about the risk factors and the importance of PAP test screening (95.9\%) but despite being in a centre with direct access to screening $69.4 \%$ of the study subjects never had a Pap test. Compared to this screening for diabetes was $74 \%$ and hypertension was $68 \%$ which was much higher.

Conclusion: The relatively higher uptake of screening for diabetes and hypertension compared to cervical screening amongst health workers demonstrates that the acceptance for invasive cervical screening perceived to be associated with sexual activity is very low. Given the magnitude of the problem, it is imperative that effective screening strategies address these sociocultural barriers also apart from the logistical aspects to decrease the mortality of this preventable cancer.

\section{Abstract \#161: Sharing the Experience in Mass Screening Programs}

Bharati Abhyankar

Purva Hospital, Sykes Extension, Takala, Kolhapur, Maharashtra, India

Aims: To screen women from remote areas devoid of basic medical facilities and to offer them treatment and guidance for follow-up.

Methods: Remote areas with no basic medical facilities were selected. Detailed information of the camp was given via pamphlets and brochures and the same were distributed by school teachers and gram sevaks. Registration was mandatory for attending the camp. The methods included were-Screening for cervical cancer by Pap smear and VIA, screening for breast cancer by clinical examination and Medical check-up and estimation of $\mathrm{Hb}$. The camps were conducted consecutively for four years and the comparison of results was done. Results: The results were compared between the years 2015-2018, and it was observed that in the year 2018, maximum turnout of 
patients among the registered, was noted. In the year 2018, highest percentage of $22.2 \%$ abnormal Pap smears were noted.

Conclusion: Lot of motivation is needed for women to undergo screening and it must be accompanied by medical check-up investigations and distribution of medicines for better prognosis. Addition of VIA testing was helpful in diagnosing and treating the patients. Screening and treatment must be adapted, keeping in check the medicolegal issues faced by private practitioners and hence the immediate need for solution is necessary.

\section{Abstract \#239: Strategy of cervical cancer prevention in Jharkhand}

\author{
Atima Bharti, Indrani Dutta \\ Department of Obstetrics and Gynaecology, RIMS, RANCHI, \\ Chhattisgarh, India
}

Objectives: To offer screening for cervical cancer to all women 30-50 years of age using visual inspection with acetic acid and create awareness among women for cervical cancer screening.

Design: Cross-sectional survey.

Methods: Women between 30-50 years of age who visited a camp organized for screening were included. Visual inspection of the uterine cervix after application of $3-5 \%$ acetic acid (VIA) was carried out. Positive cases were sent to the community health centre for colposcopy, cases for biopsy were referred to district hospital or to the nearest tertiary centre. Cases eligible for cryotherapy were treated by "SEE AND TREAT" approach.

Results: Among 100 women screened, eight patients were VIA positive, six of them were eligible for cryotherapy, two patients were sent for colposcopy-directed biopsy. Women treated with cryotherapy were called for screening after one year and VIA negative women were counselled to come after 5 years.

Conclusion: In a population of 1000, age group over 30 years is about $37 \%$ (census 2011). Screening can prevent, morbidity and mortality associated with cancer cervix.

\section{Abstract \#269: A Survey of Knowledge of HPV infection, HPV vaccination and Cervical cancer screening in female college students}

Mamta Meena, Bharti Saxena

Department of Obstetrics and Gynaecology, GMC Kota, Rajasthan, India

Objectives: Cervical cancer is the second most common cancer of women in India. Cervical cancer is preventable with organized HPV vaccination and cervical cancer screening programme. To assess the knowledge of HPV infections, HPV vaccination and cervical cancer screening programme.

Methods: It was a cross-sectional study conducted in two colleges in New Delhi. A total of 310 students of age 18-25 years participated in the study.

Result: $82 \%$ of college students had adequate knowledge regarding HPV Infection, HPV vaccination and Cervical cancer screening. Source of information for these participants were teachers in $50 \%$, parents in $20 \%$ and media and friends in rest $28 \%$. Only $2 \%$ credited family physician and gynaecologists for information in this subject. Only $10 \%$ of participants thought that they are not at risk of developing cervical cancer. Approximately $90 \%$ of candidate knew then cervical cancer is caused by HPV which is transmitted sexually. Only $5 \%$ of participants had anybody screened for cervical cancer in family and only 2 students actually had vaccination against HPV.
Conclusion: Success of HPV vaccination and cervical cancer screening program depends upon the awareness in the target group. We recommend active participation of stakeholders like gynaecologists, family physicians, NGO, teachers and parents to promote awareness and facilitate HPV vaccination and cervical cancer screening.

\section{Abstract \#326: A Single-Center Experience of Cervical Cancer Screening in an Urban Private Hospital of Telangana State}

Tanvir, Meeta, Akanshi, Anusha

Telangana, Andhra Pradesh, India

Objective: To investigate the demographic features with pap smear results in women with poor health index.

Design: Retrospective study based on hospital data.

Methods: The cervical screening was conducted at an urban private hospital. Data was collected between 2020-21, involving 561 women of $>25$ years of age with a poor wealth index from an urban population. Demographics, gynaecological and obstetrical history were recorded. A conventional pap smear test was performed. Descriptive analysis was performed and where appropriate, number and percentage is reported. Mean, and the standard deviation was presented for continuous data after the test of normality was performed. Comparison of two variables was done using the Chi-square test. A p value of less than 0.05 was considered statistically significant for the study. Results: $83.4 \%$ (468) of women agreed to a pap smear test. The prevalence of precancerous lesions was found to be $2.3 \%$-ASCUS (Atypical squamous cells of undetermined significance) in 6, LSIL (low-grade intraepithelial neoplasia) in 2, and HSIL (High-grade intraepithelial neoplasia) in 3. Age of coitus $<=18$ years was present in $51.3 \%$ (ASCUS $=5$, LSIL $=2$, HSIL $=3, \mathrm{P}=0.8$ ) among this population. $25 \%$ of women had their first child before 20 years of age (ASCUS $=4$, LSIL $=1$, HSIL $=0, P=0.43$ ). Some women had addictions -19 to alcohol (4.05\%), 10 to tobacco (2.1\%), 1 smoking $(0.2 \%) .60 .2 \%$ (282) women accepted that their food was low in fruits and vegetables (ASCUS $=3, \mathrm{LSIL}=1, \mathrm{HSIL}=3, \mathrm{P}=0.5$ ). $51.9 \%$ (294) of women were sexually inactive (ASCUS $=2$, LSIL $=1$, $\mathrm{HSIL}=1, \mathrm{P}=0.86$ ). None of these variables was found to have statistically significant association with the presence of precancerous lesions. $2.79 \%$ (13) had a Pap smear done in the last 3 years, and $0.64 \%$ (3) took HPV vaccination. The mean waist circumference was102.6 in women with HSIL and it was found to be statistically significant ( $p<0.025$, ANOVA). Other demographics were not significantly correlated with cytology reports. No cervical cancer was found. Per vaginal examination showed erosion in 43 women (ASCUS $=1$, HSIL $=1$ ), an ulcer in $1(\mathrm{HSIL}=1)$, polyp in 13 $($ LSIL $=1)$, and cervicitis in 16 . The Pearson Chi-Square showed a positive correlation of per/speculum findings with the pap results $(\mathrm{p}=0.00)$.

Conclusion: The demographics did not seem to have a major impact on the pap smear results. Although the mean waist circumference correlated with the HSIL, but the numbers are too low to draw an inference.

\section{Outreach Activities in Cervical Cancer Prevention}

Abstract \#14: A novel concept: mother-daughter pairs-'screen the mother-vaccinate the daughter'

Leela Digumarti, Priyanka Vemanamandi, HemalathaTsodury, Satyavathi Vabbalareddi, Nagendra Galla, Raghunadharao Digumarti Homi Bhabha Cancer Hospital \& Research Centre, Visakhapatnam, India 
Objectives: To achieve WHO global targets in screening and vaccination for cervical cancer elimination.

Methods: It is a novel concept in collaboration with two charities. Started in August 2019.

Study design: Support was sought from charities/NGOs/Corporates for school-based HPV vaccination of girls along with screening of mothers for cervical cancer. An awareness program was conducted in each school. Parental consent was obtained. Girls were invited in batches along with their mothers to the hospital. Quadrivalent vaccine was given to the girls and their mothers had co-testing with LBC and HPV. Vaccination cards are given. Data is electronically maintained.

Social media is used to spread the message about the project. Results:

School 1 (Aganampudi):

All the 73 girls studying in the 10th class are enrolled. There were 35 girls who were 14 years old, while 38 girls were $\geq 15$ years. In 67 girls, all the requisite doses of HPV Vaccine were completed in spite of COVID-19 pandemic. In 6 girls, only 2 doses were completed; the 3rd dose was missed as they left for their native place following closure of school.

All the 73 mothers were above 30 years of age. None had abnormal results on co-testing with LBC and HPV.

School 2 (Appapuram):

All the 61 girls studying 6th standard to 10th standard in a village school are enrolled. Majority of the girls (37/73) were in the 10 to 14 years age group. There were 24 girls who were 15 years or above. All the girls completed the requisite doses, in spite of the Covid 19 pandemic. Fifty-six mothers were screened. Only 1 woman had an abnormal Pap smear with ASCUS but negative for HPV. She is on follow-up.

Conclusions: Creation of awareness in mothers and daughters simultaneously had a greater impact on screening and vaccination. This novel concept achieves two of the three objectives of WHO's targets for elimination of cervical cancer. Collaboration with charities and non-governmental organisations with funding from corporates under corporate social responsibility can help eliminate cervical cancer in India.

\section{Abstract \#120: Cervical cancer screening model in low-resource setting using visual inspection under acetoacetate and lugol's}

Preeti Yadav, Sonal Bathla, George Verghese, Anju Bala

Department of Obstetrics and Gynaecology, Sant Parmanand hospital, Delhi India

Objectives: A camp-based, two-phase model approach was used for raising awareness and conducting screening for cervical cancer in local population in Manali, India.

Methods: As part of phase one awareness talks were organised in schools to spread awareness and encourage women to participate in screening in phase 2 . Local healthcare workers were given information for further door-to-door awareness. In Phase 2, 226 women aged 21 to 65 years presenting to the camp were screened with per speculum examination and visual inspection after application of acetoacetate and lugol's iodine (VIA/VILI). 7 women were found to be positive for suspicious lesions and further underwent excision of lesions with electrosurgery followed by histopathological examination. 1 patient turned out to be positive for high-grade cervical intraepithelial neoplasia who underwent hysterectomy on follow-up. Further camps are proposed to cover the whole population in the area. seventy girls were vaccinated with HPV vaccine, which was funded by corporate social responsibility ONGC.

Results: The venture noticed active participation of healthcare volunteers and women from the area. The skill need to go to the distant rural areas with difficult terrains, where the local healthcare workers can be trained in cervical cancer screening by VIA/VILI in camp setting to ensure continued screening periodically.

Conclusion: The same model can be adapted in each district on a regular basis to extend outreach of the cervical cancer screening and reduce the burden of treatment of cervical cancer in later stages.

\section{Abstract \#188: Opportunistic Screening During Antenatal Visits: A Step Towards Cervical Cancer Elimination}

\section{Ritu Sharma, Ruchi Verma}

Department of Obstetrics and Gynaecology, Government Institute of Medical Sciences, UP, India

Objectives: India accounts for nearly one-third of the global cervical cancer deaths. In India, it is the second most common cancer-related mortality in women. Since HPV infection is the precursor of cervical cancer in $99 \%$ of females and has a long latent phase, detecting this infection in the young females can play a great role in eliminating the cervical cancer. Taking into account the extremely low screening among young women in India, we undertook this pilot project to utilize the opportunity during antenatal contact to motivate them for screening. To find and compare the incidence of abnormal cytology and human papillomavirus (HPV) infection via co-testing among pregnant and non-pregnant women.

Methods: This prospective observational study was performed at tertiary care centre in North India. The willing pregnant women irrespective of period of gestation (group A) and equal number of agematched non-pregnant sexually active women (Group B) underwent co-testing. In case of abnormal results, colposcopy examination was performed. Antenatal females with abnormal results were followed up 6 weeks after delivery.

Results: A total of 120 women were recruited in the study with 60 in each group. Ten (16.66\%) women in Group A and $8(13.33 \%)$ women in group B were screened positive for HPV infection $(16,18)$. Among HPV positive women in group A and B, cytological abnormalities were reported among 6 and 8 women, respectively, with 4 women in each group reported as ASCUS; 2 and 3 women reported as LSIL, respectively; and HSIL was reported in one woman of group B. Out of 4 pregnant women (Group A) with ASCUS, 2 (50\%) showed regression postnatally $(\mathrm{p}<0.01)$. In group $\mathrm{B}$ the cytology of one HPV negative woman reported as ASCUS. Colposcopy findings in 2 women with LSIL from group A and 3 women with LSIL from group $\mathrm{B}$ were consistent with CIN 1; while in group B one woman with HSIL had findings consistent with CIN 2. One woman with ASCUS from each group had leucoplakia.

Conclusions: We concluded from the study that pregnant women are at higher risk for HPV infection in comparison to non-pregnant women with significant regression of positive findings in postnatal period. We would like to emphasise that HPV screening may be used as a primary screening method and antenatal period should be used as an opportunity to spread awareness and improve the screening among young females. This can play a pivotal role to achieve the $70 \%$ screening target set by WHO in eliminating the cervical cancer.

\section{Abstract \#191: Why Women with Advanced Carcinoma Cervix Do Not Seek Medical Help Early: A Tertiary Hospital-Based Indian Study}

Anoosha K Ravi, Aastha Raheja, Krishna Agarwal

Department of Obstetrics and Gynaecology, AIIMS, Rishikesh, India 
Objectives: Objective of this study was to understand the reason for delayed approach to medical help in women with advanced carcinoma cervix.

Design: It was a cross-sectional study.

Methods: Information obtained from women with carcinoma cervix clinical stage IIb and above with proven histopathological diagnosis was recorded in the predetermined format.

Results: Almost all the subjects were illiterate and were from lower socioeconomic strata and rural areas. None of them knew about the disease and its symptomatology. About $82 \%$ of the patients were symptomatic since 5-10 years, however, took no medical care during the period and 2/3rd of them did not even reveal their problem to any of the family members. About $79 \%$ of the subjects had never used any contraception and none had knowledge about its role in preventing infection. Majority of them (98\%) had no idea about the role of Pap smear in screening carcinoma cervix. All of them said if they had knowledge about the disease, they would have sought medical help much earlier.

Conclusions: The most important reason for delayed approach in our study was found to be lack of knowledge about the disease. Therefore, till such time that we have national screening program in place, these women who are at high risk of developing carcinoma of cervix should be targeted and educated through mass media so that these women could seek medical help in early stages.

\section{Abstract \#328: To Assess The Awareness Regarding Cervical Cancer Screening and Cancer Cervix Amongst Upcoming Doctors (MBBS Students).}

Manjeera Siva Jyothi, Saritha Shamsunder, Monika Gupta, Sunita Malik

Department of Obstetrics and Gynaecology, VMMC and Safdarjang Hospital, New Delhi

Objective: India accounts to around 1/4th of global burden for ca cervix. It is the 2 nd most common malignancy (3.5\%) in women in India only after ca breast (28.6\%). The estimated incidence is 570,000 cases and 311,000 deaths in 2018 globally. It ranks as the fourth most frequently diagnosed cancer and the fourth leading cause of cancer death in women. The most important factor is lack of awareness and accessibility of screening.

Present study is aimed to get an idea regarding the knowledge present in MBBS students so that strategies regarding cancer control programmes and to create awareness about Ca cervix could be devised. Methods: Seventy-nine MBBS students were given a pre-structured questionnaire as a pre-test. All the proformas were in English in medical terminologies which could be easily understood by all the students. In case of difficulty, questions were explained to individuals. Filled proformas were collected before commencing the session. Individual queries were dealt at the end of the session. The same questions were asked after the session as a posttest and the percentage of correct answers given in the pre- and posttest was evaluated.

Results: A total of 78 students have been evaluated. The percentage of female students counselled by medical professionals for vaccination was $38.2 \%$. But the vaccination rate was only $17.6 \%$. The range of correct answers in pretest value was $2-23$ with a median of 13 . The range of correct answers in post-test was 10-24 with a median of 20 . The average percentage increment between them was66.46\% (. p value $<0.0001$ ) which shows such kind of sessions are necessary to bring down the incidence of CA cervix.

Conclusion: There was a significant improvement in the knowledge attained regarding cervical cancer prevention with a counselling session.

\section{Dissemination and Communication Research}

Abstract \#266: Cervical cancer awareness among the rural population in Gautam Buddh Nagar district, India and its association with demographic factors and internet usage

Ruchika Gupta, Kavita Yadav, Roopa Hariprasad, Malasha Kumari, Sanjay Gupta, Shalini Singh

ICMR-National Institute of Cancer Prevention and Research, Noida, Uttar Pradesh, India

Objective: To evaluate the cervical cancer awareness among the population in rural areas of a district of north India and its association specifically with internet usage.

Design: Cross-sectional house-to-house survey.

Method: Households in three villages of a district of the national capital region of Delhi were selected using a stratified random sampling technique. A pretested questionnaire-based survey was conducted among adults $(n=204)$ in the selected households on various aspects of cervical cancer awareness. The data were analyzed for the association with socio-demographic factors and internet usage. Results: Though the majority (96.5\%) of the participants were aware of cancer, the specific risk factors and screening tests for cervical cancer were not known to a vast majority $(79.9 \% \& 77.4 \%$, respectively) of participants. Awareness of risk factors of cervical cancer and use of HPV vaccine for prevention of cervical cancer correlated positively with the education level of the participants. Internet users who specifically sought health information were more aware of aspects such as cancer screening for prevention, and use of HPV vaccine for cervical cancer prevention when compared with the general internet users.

Conclusion: The present study highlights the low level of awareness of cervical cancer among the rural population of Gautam Buddh Nagar district. The association of cervical cancer awareness with health information-seeking internet usage may potentially be exploited for community penetration of cancer awareness campaigns and programs through internet-based platforms.

\section{Public Response in Mass Screening}

Abstract \#116: Cervical Cancer Screening: Knowledge, Attitude and Practices among Nursing Staff attached to a Rural District Hospital in South India

Rahul Anand

Government Kamraj Hospital, India

Objectives: To assess the knowledge, attitude and practices about cervical cancer screening among the staff nurses attached to a rural district hospital.

Methods: This was a descriptive cross-sectional study conducted in the rural district hospital in Chidambaram, Tamil Nadu, from November to February 2020. A total of 200 staff nurses attached to the hospital (Headquarters, Taluk, Primary Health centres) were enrolled in this study.

Results: Only $15 \%$ had undergone cervical cancer screening. $35 \%$ of the nursing staff did not even know a single risk factor for cervical cancer. Only 5\% had regular Pap smears according to the guidelines. Not considering themselves to be at risk was the top reason (70\%) for not undergoing the screening. Feeling of shame and shyness to proactively seek a cancer screening was the second reason $(15 \%)$. None of them had received HPV vaccine. Only $15 \%$ had vaccinated their daughters.70\% did not have sufficient knowledge about the HPV vaccine $35 \%$ believed that the vaccine was more of a fad and not very common. $80 \%$ stated that they would not recommend that HPV vaccine to their patients. 
Conclusion: The knowledge about cervical cancer screening, HPV, and its causal association with cervical cancer and the HPV vaccine was surprisingly very low among the staff nurses.

\section{Abstract \#254: Cervical Cancer Screening: Knowledge, Attitude and Practices Among Nursing Staff in a Tertiary Care Hospital of North India}

Nishu Bhushan, Nivedita Prashar

Government Medical College and Associated Hospitals Jammu, India

Introduction: Cervical cancer is one of the major concerns of public health importance in today's world. Cervical cancer-related deaths among women in India are often due to late diagnosis of the disease. Knowledge about cervical cancer and early screening is the most effective measure for its prevention.

Objective: To assess the knowledge, attitude and practice of nursing staff about cervical cancer screening in a tertiary care hospital of north India.

Methods: It was a cross-sectional, descriptive, interview-based survey conducted at Government Medical College and Associated Hospitals Jammu with a pre-tested questionnaire among 230 nurses between January 2019 to March 2019.

Results: In the present study, it was seen that $81.7 \%$ of the nurses knew that Pap smear is used for screening of cervical cancer but only $47.5 \%$ knew that Pap smear can also detect pre-cancerous lesions of cervix. Only $27.8 \%$ knew that human papillomavirus infection is a risk factor for cervical cancer. $72.5 \%$ of the participants knew that early cervical changes are easily curable. $33.6 \%$ reported that women $\geq 21$ years of age or those who are sexually active for the last 3 years (whichever is earlier) should undergo screening. $91.3 \%$ of the nurses were aware of cervical biopsy as a diagnostic modality other that Pap smear. Only $24.5 \%$ of the nurses were aware of HPV vaccination. Only 20 nurses had themselves screened by Pap smear. 91.3\% of the nurses had never taken Pap smear of any patient.

Conclusion: The majority of nursing staff may have inadequate knowledge about cervical cancer screening and their attitude and practices towards cervical cancer screening could not be termed positive.

\section{Abstract \#256: A Cross-Sectional, Hospital-Based Study: Knowledge, Attitude and Practice Towards Cervical Cancer Among Women Attending Obstetrics and Gynecology Department}

Aakriti Manhas, Nikita Gandotra

GMC Hospital Jammu

Background: Cervical cancer is one of the most common malignancies among women of reproductive age group. It has now been proven that HPV is the major causative factor of carcinoma of the cervix. The most propitious feature of this cancer is that it is preventable and curable in the early stages.

Objectives: To assess the knowledge, attitude and practice towards cervical cancer among the women.

Methods: A total of 300 women were enrolled and subjected for faceto-face interview using a questionnaire on cervical cancer. This is an outpatient-based cross-sectional study done between June 2019 to July 2019.

Results: Out of 300 females $152(50 \%)$ knew about the cancer. 92 $(30 \%)$ heard from the media and $49(16 \%)$ heard from friends. 120 $(40 \%)$ knew about the risk factors \& $129(43 \%)$ had knowledge about symptoms. 117 (39\%) had awareness about preventive measures and $97(32.3 \%)$ knew about the screening modalities.
Conclusion: There is a need to make the general population aware about the risk factors of cervical cancer and proper screening should be done to prevent the development of cervical cancer. Proper campaign and programs should be organized in rural areas towards the same end.

Abstract \#304: Assessment of Knowledge, Attitude and Practices Gap regarding Cervical cancer screening amongst female doctors in Tertiary Care Hospital of Southern Rajasthan

Nalini Sharma

Geetanjali Medical College and Hospital

Objectives: In recent years, focus of cervical cancer prevention efforts has shifted from secondary prevention to primary prevention. The pertinent question is the providers of screening, those who understand the magnanimity of the problem globally and locally why do not they themselves get screened. Living in hygienic urbane conditions, good contraceptive coverage, having no family history of cervical cancer, awareness about clinical symptoms-does it give them security to defy globally routinely recommended guidelines regarding screening? We wish to elucidate the current situation and spotlight the roadblocks to routine cervical cancer screening as felt by female doctors.

Methods: A cross-sectional study of female doctors (including residents) working in Geetanjali Medical College and Hospital, initiated after approval from institutional research review committee and institutional ethical committee. After informing the purpose of study, printed/online questionnaire (semi-structured) was administered. Questions comprised of whether they themselves got screened, knowledge and attitude about screening and reasons for not getting themselves screened.

Results: A total of 123 responses were recorded. $78.4 \%$ of the female doctors were working at medical college level. $96.8 \%$ were postgraduate students or postgraduates. Majority $(95 / 76 \%)$ were in age group 25-39 years, $15.2 \%$ (19) in 40-54 years age group. Majority were aware that age of starting coitus, multiple sex partner and family history of the same are main risk factors.

Lack of knowledge was observed regarding correct age of screening, HPV DNA testing, whether women over 65 can discontinue screening. $94.4 \%$ of the female doctors believed in mandatory cervical cancer screening; however, only $30.4 \%$ got themselves screened. $50 \%$ out of them (19/38) were regular with their screening. A meagre six doctors have been screened for cervical cancer more than three times. Barriers recorded were they have not had any complaints $(58.4 \%)$, never thought of it $(36.8 \%)$ and discomfort during external examination $(31.2 \%)$ as the top three. $63.2 \%$ has not been vaccinated for cervical cancer. $70.45 \%$ (31/44) of those who were were vaccinated after being sexually active.

Conclusion: A startling gap in knowledge, attitude and practices prevails even in female doctors. What female healthcare professionals practice, they will advise to masses. Barriers should be addressed to achieve the vision of cervical cancer-free world.

\section{Attitude of Society Towards HPV Vaccination}

Abstract \#114: Gap analysis between awareness and acceptance of HPV vaccination among undergraduate female students in suburban area in India

Ritu Sharma, Rakhee Sharma

Department of Obstetrics and Gynaecology, Government Institute of Medical Sciences, India 
Objectives: To analyze the gap between awareness and acceptance of HPV vaccination among undergraduate female students.

Methods: This cross-sectional study was conducted after institutional ethical clearance among undergraduate female students in the age group of 17-26 years. Considering 21\% HPV awareness, power of $80 \%$, and a standard error of $5 \%$, the estimated sample size was 266 . A pretested questionnaire was used which apart from information regarding socio-demographic profile included questions related to the knowledge about HPV infection, HPV vaccination and factors influencing its acceptance.

Results: Though the awareness of HPV infection and HPV vaccination was good in medical student subgroup $(\mathrm{p}<0.05)$, the overall awareness among participants was poor. Among the participants who were aware of HPV vaccine, only $10 \%$ were vaccinated; the most important determinants for this gap being the cost and myths related to its safety and efficacy. Further among all unvaccinated participants, $48.54 \%$ were not willing to take the vaccine. The main reasons for non-acceptance were cost (38\%), lack of awareness (40\%), dilemmas regarding safety $(7 \%)$ and efficacy $(10 \%)$.

Conclusion: Since almost all the determinants related to non-acceptance of HPV vaccine, except cost, can be eliminated by spreading awareness, a dedicated HPV vaccination awareness program should be implemented to clear the myths and support the facts in order to augment its acceptance. Further, instead of keeping HPV vaccination optional, it should be incorporated in routine National immunization program and integrated with national cancer control program to meet the financial aspect.

\section{Abstract \#137: Knowledge of cervical cancer, HPV infection and vaccine among college students in north India}

Rajesweri Ponniah, Archana Mishra, Saritha Shamsunder, Anita Sabharwal

Department of Obstetrics and Gynaecology, VMMC and Safdarjung Hospital, New Delhi. India

Objectives: Human papillomavirus (HPV) infection is associated with a number of diseases like Genital warts, Anal warts, premalignant and malignant lesions of cervix. Cervical cancer is the second most common cancer among women in developing countries. To assess the knowledge of college students on cervical cancer, HPV infection and vaccination.

Methods: Present study is undertaken to assess the knowledge of cervical cancer screening, HPV infections, and HPV vaccination among college students. This done by structured questionnaire with multiple-choice, dichotomous and open-ended questions among 90 college students and their knowledge on cervical cancer and human papillomavirus infection assessed.

Results: Most students had adequate knowledge regarding cervical cancer and HPV virus. But many were ignorant (89\%) of the frequency of pap smear screening among reproductive women. Students were ignorant $(100 \%)$ especially about the type of lesions it causes like genital warts, penile cancer and oral cancer other than causing cervical cancer. Almost $66 \%$ of students have adequate knowledge about the sexual transmission. Only $34.4 \%$ knew that HPV vaccination to be given to teenage girls. Almost $96.6 \%$ believe that there is no need of pap smear after vaccination.

Conclusions: Though there is adequate knowledge, there are focal areas where more knowledge is required in order to have effective attitude towards HPV infection, cervical cancer and vaccination.

\section{Abstract \#155: HPV Vaccine Refusal-Reasons Analysed}

\section{Namoijam Basanti, Aruna Nigam, Sumedha Sharma}

Hamdard institute of medical sciences and research, South Delhi, India

Objectives: Cervical cancer is the most common gynaecological cancer among women in India. Multipronged approach for its management is the need of hour, i.e., screening and treatment of preinvasive lesions, early diagnosis and HPV vaccination. To find out the awareness of HPV vaccine and willingness to accept the vaccination after imparting information on it.

Methods: It was a cross-sectional hospital-based study conducted in a tertiary care hospital in South Delhi. A pre-designed, pre-tested closed-ended questionnaire regarding KAP of cervical cancer and its vaccination was administered to patients attending the Gynaecology OPD.

Results: The total number of participants was 200. Most of them (58\%) belonged to 21 to 30 years of age. Despite having good educational background ( $40 \%$ were graduates), only $21 \%$ have heard of cervical cancer, $7 \%$ of them knew about the HPV vaccine and only 3 people $(1.5 \%)$ had got vaccinated. There were nine nurses in the study group, out of which 4 (44\%) had no knowledge about the HPV vaccine. Even after being given information regarding the vaccine, $54 \%$ of the population refused vaccination $34 \%$ on account of lack of adequate knowledge, $8 \%$ because of non-affordability.

Conclusions: There is an urgent need to decrease the KAP gap regarding HPV vaccination so as to fight successfully against cervical cancer and achieve the WHO goal of 2030 regarding cervical cancer elimination.

\section{Abstract \#165: Strategies to increase acceptance of human papillomavirus vaccination}

Kaur $\mathrm{R}^{1}$, Sharma $\mathrm{H}^{1}$, Goel $\mathrm{B}^{1}$, Takkar $\mathrm{N}^{1}$, Walia $\mathrm{D}^{2}$, Prinja $\mathrm{S}^{2}$

Department of Obstetrics and Gynecology ${ }^{1}$, Department of Preventive and Social Medicine ${ }^{2}$

Government Medical College and Hospital, Chandigarh, India

Background: The lifetime risk of developing cervical cancer in Indian women is $2.5 \%$. The study focuses on assessing the factors affecting uptake of HPV vaccination by assessing their knowledge, belief and attitude of women attending the gynecological outpatient department.

Methods: A prospective study was performed from 2017-2019 in the department of gynecology with a sample size of 503 women. The study subjects attending gynecological OPD for any complaint with at least one daughter who was eligible for HPV vaccination were interviewed using pretested structured validated 42 item questionnaire with open- and close-ended questions.

Results: Awareness of cervical cancer was seen in $200(39.8 \%)$ women, 109 (54.5\%) women knew cervical cancer was preventable and awareness about HPV vaccine was seen in $26(5.2 \%)$ women. The desire for HPV vaccination was shown by $69.8 \%$ of women after counselling. On logistic regression analysis knowledge regarding cervical cancer and HPV showed significant correlation with poor knowledge regarding contraception, younger age ( $<35$ years) and rural background. Husband Played a major role in more than $50 \%$ cases for making decision on behalf of their wives for any health-related issues. The average distribution of coverage of routine childhood vaccination was $97.6 \%$ and optional vaccine was $22.3 \%$. The reasons cited for not taking optional vaccine was nonrecommendation by the doctors in $90.8 \%$ cases and cost of vaccine being high in $(7.9 \%)$ cases. 
Conclusions: The role of physician and health system in spreading awareness and increase in acceptability for HPV vaccine needs careful consideration in developing countries.

\section{Abstract \#235: Human Papillomavirus Vaccine Uptake Amongst Children of Gynaecologists in Northern Nigeria}

Aisha Mustapha, Afolabi KoredeKoledade, Rabiat Aliyu Muhammad Department of Obstetrics and Gynaecology, Ahmadu Bello University / Teaching Hospital, Zaria, Nigeria

Objective: Cervical cancer is the commonest genital tract malignancy in northern Nigeria. The World Health Organization has strategized that to be on the path towards cervical cancer elimination by the year 2030 , $90 \%$ of girls must be fully vaccinated with human papillomavirus (HPV) vaccine by age 15 years. Coverage of HPV vaccination is still very low as only individuals who are aware of, and can afford the vaccine, give their children. Gynaecologists are expected to meet these two criteria. This study therefore aims to assess the HPV vaccine uptake amongst children of gynaecologists in northern Nigeria.

Design: A descriptive cross-sectional multi-institutional questionnaire-based study.

Method: Self-administered questionnaires were served to 198 consenting gynaecologists at a northern zonal conference who had children 9 to 15 years.

Results: The mean age of respondents was $42.6 \pm 8.2$ years. Fiftythree percent were males, $42.7 \%$ were females. Majority $(64 \%)$ were consultants. Even though $96.1 \%$ of the respondents would consider vaccinating their children, only $12.9 \%$ had actually given at least one dose of the vaccine to their eligible children, with $92.3 \%$ of these being consultants. Reasons given for non-vaccination include lack of access in $19.4 \%$ followed by high cost in $14.6 \%$ of cases. There was a statistically significant positive correlation between higher cadre doctors and children's HPV vaccination uptake. Seventy percent were willing to vaccinate both males and female children.

Conclusion: Only $12.9 \%$ of Gynaecologists vaccinate their children and a significant $14.6 \%$ can't afford it.

\section{Abstract \#250: HPV Vaccination Programmme-Hurdles \& Challenges in a Tertiary Care Centre \\ Pratibha Kumari, Sangeeta Pankaj \\ Indira Gandhi Institute of Medical Sciences, Patna, Bihar, India}

Objective: This study was done to explore reasons behind acceptance of HPV vaccine among parents who came for HPV vaccination in a tertiary care hospital. HPV is the most common STI worldwide, infecting 3 of 4 individuals at least once in lifetime. This virus exists in $>200$ morphogenic strains and some of these are oncogenic. HPV is detected in virtually all cases of cervical carcinoma. So, HPV vaccination against high-risk HPV types is expected to reduce burden of cervical cancer.

Methods: In May 2018, HPV immunization program was started in Gynaecology oncology department, IGIMS, PATNA-a tertiary care centre of Bihar for girls of medical staffs and their relatives of age group 9-26 years. Bivalent Cervarix vaccine was used for vaccination. Questionnaire including the reason for acceptance for vaccine and from where they got knowledge of HPV vaccination was enquired both to parents and daughters. Total 60 girls got vaccinated under this program.

Result: Parents of HPV vaccine receiving girls have knowledge about cervical cancer but they lacked in knowledge regarding vaccine and that HPV is the causative agent of cervical cancer. Young adolescents girls were not aware of this vaccine either.

Conclusion: Three-dose schedule was a major barrier to the delivery and uptake of the HPV vaccine. Had a single dose of HPV vaccine been effective, that would have been important logistics advancement. Lack of knowledge about vaccine is a problem that leads to low vaccination coverage. Government of India should introduce chapters related to cervical cancer and its vaccination in curriculum of high schools so that both parents and their children become aware of this deadly entity and its preventive measures in form of Cervarix/ Gardasil.

\section{Abstract \#291: Barriers to compliance for cervical screening among HPV vaccinated women in low-resource settings}

Anju Singh, Shachi Vashist, Rajesh Kumari, Swati Tomar, Sarita Kumari, P. Vanamail, Neerja Bhatla

Department of Obstetrics \& Gynaecology, All India Institute of Medical Sciences, New Delhi, India

Objective: To evaluate compliance with cervical screening after HPV vaccination and to determine barriers, if any.

Design: Prospective cohort study.

Method: In 2010, 1000 unmarried girls aged 10-18 years received $\mathrm{qHPV}$ vaccination as part of an Indian multicentric trial to evaluate two versus three doses of HPV vaccine. Cervical sampling was advised 18 months after marriage or 6 months after delivery, whichever was earlier. Cervical samples were collected at the health centre. Participants who did not attend were offered home collection with option of self-sampling.

Results: Of 463 participants who got married, 409 (88.34\%) were eligible for first cervical sample. Among these, 334/409 (81.66\%) complied with screening: at centre $(\mathrm{n}=171,51.19 \%)$; at home ( $\mathrm{n}=163,48.81 \%)$. Only $2(0.59 \%)$ came for screening on their own, $29(8.68 \%)$ were motivated telephonically, $256(76.65 \%)$ after personal counselling, and $47(14.08 \%)$ required both telephonic and personal counselling. Among the non-compliant, 36 refused (self/family member), 25never returned after marriage, 9 were pregnant, 3 because of corona pandemic, 1 was on treatment for infertility, and 1 cited duty hours. None accepted self-sampling.

There was a significant difference in mean age at marriage of compliant versus non-compliant participants $(20.02 \pm 2.7$ vs $22.39 \pm 2.5 ; \mathrm{p}=<0.01)$ On univariate analysis, higher age at marriage $(\mathrm{OR}=0.72, \mathrm{p}=0.001)$ and higher education level of participant $(\mathrm{OR}=0.31, \mathrm{p}=0.024)$ and husband $(\mathrm{OR}=0.113$, $\mathrm{p}=0.036$ ) decreased compliance with screening after vaccination.

Conclusion: Awareness and reinforcement are even more important among educated participants to increase compliance with cervical screening post-vaccination. Self-sampling is not easily understood by the community.

\subsection{Quality Assurance in Cervical Screening and Colposcopy}

\section{Abstract 11 \#: Rate of overtreated cervical lesions in Ukraine \\ Dr. Iryna Muryzina, Kharkov National Medical University, Ukraine}

Background: Despite HPV test significance in recognition, CIN2 + low specificity seeds unnecessary anxiety among HPV-positive women with reassuring cytology and colposcopy and requires triage and surveillance with lingering uncertainty how to manage 
cases of LSIL and persistent HPV-infection. It is estimated that $30 \%$ women with LSIL undergo loop procedures (LEEP) that does not comply with current guidelines not to treat LSIL. In Ukraine, this number might be higher, because of failure to implement registry for LSIL and quality assurance for precancerous lesions management.

Objectives: To assess compliance with recommendation against routine treatment of confirmed LSIL $(\mathrm{CIN} \leq 1)$.

Methods: Study collated referral indications (only precancerous legions) for LEEP with histological conclusion of samples from primary Gyn settings (not GynOnc).

Results: Among 378 cases, there were $64.8 \%$ (245) referrals with solely LSIL in women $<50$ years, and among them $18 \%$ (44) were treated on the site of the first colposcopy provided at the random gynaecologic visit without specific indications (without Pap smear, HPV-testing and precedent biopsy) and none of them recognized CIN2 + . 49\% (120) referrals due to persistent LSIL HPV-positive showed no discordant results of surveillance, $37 \%$ cases of treated long-lasting LSIL were hrHPV-negative. Just 2.9\% (7) of long-lasting LSIL had been HPV mRNA E6/E7 tested (positive). When LSIL referrals were collated with definitive histologic conclusion CIN $\leq 1$ constituted $94.3 \%$. Among 14 cases of CIN2 + in initially LSIL referrals there were 5 HPV mRNA E6/E7 positive.

Conclusions: Overtreatment of LSIL in Ukraine is very common. The main hazard is cessation of routine screening due to ungrounded complacency.

\section{Abstract \#13 Quality assurance in the performance of a VIA- based "screen and treat" cervical screening in low-resource areas of Telangana, India}

Usha Rani Poli, Patricia Bidinger, Mukesh Janbandhu, Swarnalata Gowrishankar

\section{MNJ Institute of Oncology \& Regional Cancer Center, India}

Objectives: A VIA-based screen and treat approach for cervical cancer using trained nurses, is an effective tool for low-resource settings. Quality assurance is crucial. We report the role of digital colposcopy with image transfer using a smart phone and WhatsApp for screen test positives or doubtful cases. A decision is then made on the course of treatment-ablative treatment according to "screen and treat" protocol as a quick quality assurance process.

Method: Eligible women between 25-60 years were screened opportunistically using the VIA test performed by nurse midwives in three district hospital OPDs from January through December 2019. All women underwent VIA test followed by digital colposcopy. The screen-positive images and from doubtful cases were immediately captured from the monitor using a smartphone and then transferred by WhatsApp to an off-site physician who offered opinion or for record purposes. Cervical biopsies were taken for histopathological correlation. Screen positive women willing and eligible for thermocoagulation (TC) were treated at the same visit.

Results: A total of 14,417 women with a mean age of 35 years were screened with overall VIA positivity of $6.8 \%$. Around $87.4 \%$ of screen positive women underwent colposcopy with image transfer. There were $81(0.56 \%)$ invasive cancers detected and $0.54 \%$ of biopsy-proven precancers. A total of 152 women were treated by TC, with 5 times overtreatment rate.

Conclusions: Digital colposcope image capture and transfer by smartphone to remote experts can strengthen the diagnostic decisions by trained nurses as an adjunct to maintain quality care for programs in low-resource rural areas.
Abstract \#22: A collaborative model to promote affordable, accessible and quality cervical cancer screening

Rajeswari Sivaprakasam

Gleneagles Global Health City, India

Objectives: Cervical cancer can be prevented if detected early. So as a need of the hour we planned a mini mobile colposcopy project. A collaborative model was planned by a mobile colposcopy unit for cervical cancer screening. The challenges faced and the outcomes were analyzed.

Methods: The mobile colposcopy unit and the Pathologist were from Chennai. The colposcopy extension clinic was done once in six weeks in a Gynaecologist Nursing Home in Cuddalore. Clinical breast examination and Colposcopy were performed. The reports were mailed to colposcopist and gynaecologist for follow-up.

Result: From May 2015-August 2018, 680 patients were given appointment for colposcopy of which $312(46 \%)$ women attended the extension clinic. The mean age of the 312 women was 39 years. Out of the 312 patients, 132 (42\%) had abnormal colposcopy findings and had directed biopsy. Of the 132 biopsies taken 30 (9.6\% of total screened) women were positive for premalignancy $(21-\mathrm{CIN} 1,8-$ CIN 2, 1-CIN 3), and $2(0.6 \%)$ were positive for malignancy.

Conclusion: The collaborative effort increased the importance given to cervical cancer screening in a routine gynaecology practice. Those with confirmed cervical abnormalities were treated and cured in their latent phase itself. Key challenges faced were poor participation and loss to follow-up of women with abnormal findings. The limitations of the project are screening availability on only fixed dates and the need to pay for services.

\section{Abstract \#47: Problem solving using the A3 methodology for colposcopy}

Priyanka Vemanamandhi, Raghunadha RaoDigumarti, Leela Digumarti, Vidhya Viswanath, Anupurva Dutta, Hemalatha Tsodury

Homi Bhabha Cancer Hospital and Research Centre, India

Objectives: Most important aspect of any screening camp (which is often neglected) is the follow-up of women with abnormal pap smear for colposcopy. Using the A3 methodology tool we aimed at improving the colposcopy coverage from a baseline of $12.5 \%$ to $95 \%$ over a period of 10 months.

Methods: With the help of Equip India QIA3 problem-solving approach, we have identified the problems leading to low colposcopy follow-up among women screened in the community. Different tools used in the A3 methodology are the Process map, the GEMBA walk, Run chart, Fishbone and Pareto chart. All these tools aided in picking out the root causes for our low colposcopy coverage. These in turn helped in achieving key drivers and interventions to address the problem. The major root causes that we derived through this methodology were delay in report generation time, attitudinal change among the volunteers and women being screened, delayed follow-up schedules. Setting up an outreach colposcopy center and developing an application which helped in viewing all the pap smear results along with color code for abnormal results were among the major interventions with high benefits.

Results: With the help of A3 methodology tools we were able to follow-up all women requiring colposcopy and sustain it $(12.5 \%$ to $100 \%$ ).

Conclusion: The A3 methodology is found to be useful and continuous quality improvement and problem-solving tool. 


\section{Abstract \#157: An audit on Colposcopy at a tertiary teaching} hospital in South India

Anitha Thomas, Ajit Sebastian, Dhanya Susan Thomas, Rachel George Chandy, Priya Bhati, Grace Rebekah, Abraham Peedicayil

Christian Medical College, Vellore, Tamil Nadu, India

Objectives: To audit the performance of colposcopy with respect to indications, results and its correlation with post-procedural biopsies using the NHS Cervical Screening Programme Colposcopy and Programme guidelines in collaboration with the British Society for Colposcopy and Cervical Pathology.

Design: Retrospective audit.

Methods: We conducted an audit of colposcopies performed for abnormal cytology smears from November 2017-June 2018. Indications for the colposcopy, findings, biopsy results, follow-up procedures done were investigated. These were then analyzed according to the International federation for colposcopy/NHS SPCP guidelines and checked for the fulfilment of criteria.

Results: A total of 14,332 Pap smears were done at this tertiary hospital during this period. Colposcopies were done for 150 of these patients. Colposcopies done for the positive high-risk HPV and Pap smear abnormality of ASCUS and above were chosen for the audit. The audit revealed compliance in 7 out of 8 criteria except for the reporting of the adequacy of the colposcopy (not achieved in $2.5 \%$ ). The correlation for the low-grade score $<6$ to low-grade biopsies $(<\mathrm{CIN}$ II $)$ and the high-grade Swedes score $>6$ to higher-grade lesions $(>/=$ CINII) was good with kappa coefficient of 0.572 and $p$ value of $<0.000$.

Conclusions: Audit of colposcopy performance using the NHSCSP guidelines revealed that compliance to guidelines was good. Correlation of colposcopy findings with the diagnostic biopsy was good. Supervised training is essential and can lead to quality improvement with fulfilment of all standards.

\section{Abstract \#184: Compliance of a Gynaecologic Oncology Centre to ESGO Surgical Quality Indicators for Cervical Cancer}

Monica Thiyagarajan, Vinotha Thomas, Ajit Sebastian, Dhanya Susan, Anitha Thomas, Rachel Chandy, Abraham Peedicayil

Department of Gynaecologic Oncology, Christian Medical College, Vellore, India

Objective: To assess the compliance of a gynaecologic oncology department in surgical management of carcinoma cervix, to the 15 quality indicators for treatment set by ESGO (European Society of Gynaecologic Oncology).

Design: Retrospective audit.

Setting: Department of gynaecologic oncology in a tertiary care centre in South India, $1^{\text {st }}$ June 2017-31st May 2020.

Methods: Electronic medical records of 82 patients with operable carcinoma cervix who underwent surgical management were retrieved and analysed.

Result: The department met the targets regarding adequate case load, training and experience of the surgeon and research. Potential candidates were counselled about fertility-sparing technique. However, despite a desired target of $100 \%$, only $46 \%$ (37/82) were planned in multi-disciplinary team meeting, $74 \%(61 / 82)$ underwent required preop investigations. As against set targets of $100 \%, 90 \%$ and $90 \%$ for essential surgical, pathological reporting and documentation of postoperative morbidity, respectively, approximately 7\% (6/82) surgical and $49 \%(40 / 82)$ of pathology reports met the requirements whereas $78 \%(64 / 82)$ had structured reporting of post-operative morbidity. There were no urological fistulas. Six (7\%) had undergone lymph node staging according to guidelines, 18\%(15/82) had involved surgical margins, $16 \%(9 / 55)$ were upstaged post-surgery as against the individual set targets of $98 \%, 3 \%$ and $9 \%$. About $10 \%$ had recurrence within 2 years (Target $<10 \%$ ), 51\% received adjuvant chemoradiation (Target $<15 \%$ ).

Conclusion: This study revealed lacunae in surgical care which required urgent addressal. Necessary changes have been implemented and periodic audit will ensure compliance and improvement in patient outcome.

Abstract \#202 Comparison and correlation of colposcopy scores with histopathology in screening positive patients

Aswathy G Nath, Rema P, Remani Wesley, Suchetha S, Sivaranjith J, Dhanya Dinesh

Regional Cancer Center, Thiruvananthapuram, India

Objectives: Colposcopy was introduced to aid cervical biopsy to detect premalignant lesions of cervix. Reid, Swede and IFCPC scores were later introduced to avoid subjective variation. To study correlation between colposcopy and histopathology in screening positive patients. To correlate Reid score, Swede score and IFCPC score during colposcopy with cervical histopathology in detecting premalignant lesions of cervix in screen positive patients.

Methods: Prospective study of patients who underwent colposcopy for cervical cancer screening positivity in the Division of Gynecologic Oncology, RCC, from June 2018 to January 2021. Reid score, Swede score and IFCPC score were documented during colposcopy in colposcopy charts. Colposcopy scorings and histopathology was statistically assessed using spearman's rank correlation test.

Results: Out of a total 220 females screened, the average age was 49.75. Colposcopy was adequate in $98 \%$ cases and biopsy taken for all cases. 51.8\% females have high-grade histopathology, and $48.2 \%$ females have low-grade histopathology. Reid score $>4$ correlated with $62.3 \%$ of high-grade histopathology $(\mathrm{p}=0.149)$, Swede score $>5$ correlated with $61.3 \%$ of high-grade histopathology report ( $p=0.038)$, while IFCPC score as major lesion correlated with $78.3 \%$ of high-grade histopathology $(\mathrm{p}=0.001)$. Reid score has sensitivity $62.3 \%$, specificity $47.4 \%$; Swede score has sensitivity $61.3 \%$, specificity $52.6 \%$ with histopathology. IFCPC scoring had sensitivity $78.3 \%$, specificity $47.4 \%$ with histopathology. Measurement of agreement kappa was 0.096 for Reid score, 0.139 for Swede score and 0.254 for IFCPC score. P value was significant with IFCPC score, 0.001 .

Conclusions: IFCPC scoring has a better correlation and more sensitivity in detecting high-grade lesion while swede score is more specific in detecting high-grade lesion.

\section{8: Think beyond disease}

\section{Quality of Life after Cervical CancerTreatment}

Abstract \#201: Life after Exenteration: Survival outcome, complications, and quality of Life Assessment in Patients after Exenteration for Gynecological Malignancies

Aswathy G Nath, Rema P, Suchetha S, Sivaranjith J, Dhanya D, Jagath Krishna

Regional Cancer Centre, Thiruvananthapuram, Kerala, India

Background: Five-year overall survival after pelvic exenteration ranges between 25 to $50 \%$. Recent developments in cancer trials as 
well as treatment approach is by giving increased importance to quality of life, so as to increase the number of long-term survivors after treatment.

Objectives: To study functional quality of life of patients after exenteration for gynecological malignancies as well as survival outcome and complications associated with such morbid procedures.

Methods: This study includes patients who underwent pelvic exenteration procedure for gynecological malignancies, from January 2006 to December 2016. Survival outcome and complications were assessed retrospectively, while quality of life was prospectively assessed from June 2018 to September 2019 during follow-up visits. Quality of life was assessed using EORTC QLQC30 and with FACIT CX questionnaire.

Results: Medium follow-up period was 87 months. $81.25 \%$ had adequate follow-up to 7 years. Out of 32 patients, 13 are alive and disease free. Median overall survival was 41 months. Five-year as well as 7 -year overall survival was $38 \%$. Median disease-free survival was 15 months. Long-term Clavein-Dindo grade 3 complications were significant. Global health status, role functioning, emotional functioning and physical functioning scores were above 70 for all patients and symptom score was less than 40 .

Conclusion: Despite significant immediate and late complications exenteration procedure is well tolerated with good QOL.

\section{Abstract \#279: A Survey of Quality of Life of Cervical Cancer Survivors in India}

Sarita Kumari ${ }^{1}$, Romey Rai ${ }^{1}$, Dipanwita Banerjee ${ }^{1}$, Rudrika Chandra $^{1}$, Rajesh Kumari ${ }^{1}$, Sushmita Pathy ${ }^{2}$, DayaNand Sharma ${ }^{2}$, Neerja Bhatla ${ }^{1}$

${ }^{1}$ Division of Gynaecologic Oncology, Department of Obstetrics and Gynaecology, All India Institute of Medical Sciences, New Delhi, India

${ }^{2}$ Department of Radiation Oncology, All India Institute of Medical Sciences, New Delhi, India

Objective: To assess factors affecting the quality of life (QOL) of cervical cancer survivors after initial treatment.

Design: Cross-sectional study.

Method: QOL of cancer cervix survivors (all stages) who received treatment and follow-up in the Gynae Cancer Clinic between January 2018 to December 2019 was evaluated by the European Organization for Research and Treatment of Cancer QOL Questionnaire Core-30 item (EORTC QLQ-C30) and Cervical Cancer Module (EORTC QLQ-CX24). Women with recurrent disease, language difficulties, or unwilling to participate were excluded.

Results: There were 61 participants with mean age $51.8 \pm 11.3$ years. Majority $(62.3 \%)$ were illiterate, with poor socioeconomic status (68.9\%), from rural areas (68.9\%); half $(50.8 \%)$ were in advanced stage at diagnosis, $86.9 \%$ received single-modality treatment. Only $14.8 \%$ were sexually active. On functional scales, $13.1 \%$ and $6.6 \%$ had problematic scores in global health status and body image, respectively. On symptom scales, all had experienced one or more symptoms, whereas $19.7 \%$ suffered from significant financial worry. Insomnia $(p=0.01)$, peripheral neuropathy $(p=0.03)$, and menopausal symptoms $(p=0.01)$ were significantly associated with age $<50$ years $(p=0.03)$; role function and financial worry were significant $<1$ year since diagnosis. Cognitive $(p=0.005)$ and emotional problems $(p=0.001)$, neuropathy $(p=0.03)$, appetite loss $(p=0.01)$ and sexual enjoyment difficulties $(p=0.02)$ were commoner if illiterate $(p=0.005)$; literate had more nausea $(p=0.05)$ and social dysfunction $(p=0.001)$. Neuropathy was significant in rural residents $(p=0.04)$. Lymphedema was more common in advanced stages $(p=0.01)$. Multiple treatment modalities resulted in more fatigue ( $p=0.004)$.

Conclusion: QOL assessment and targeted management should be routinely practiced in cancer cervix survivors.

\section{0: Other Precancerous Lesion and Cancers Related To HPV}

\section{Anal Disease}

Abstract \#5: The Availability of Experience and Expertise to Manage Patients with Multizonal Intraepithelial Neoplasia (MZIN); a BSCCP Survey of UK Colposcopists

Alice McGee, Theresa Freeman-Wang, Deirdre Lyons, Julie Bowring, Jennifer Byrom

Aberdeen Centre for Women's Health Research, United Kingdom

Objectives: Colposcopists in the UK are required to undertake training and reaccreditation to ensure the quality of colposcopy services in the management of women with cervical disease. With increasing rates of vulva, peri-anal, and anal intra-epithelial neoplasia, many UK colposcopists also see women with intra-epithelial neoplasia at these non-cervical sites and have raised queries to the British Society of Colposcopy and Cervical Pathology (BSCCP) about detection and management of non-cervical disease. There is no guidance currently available and we have no record of available services and expertise.

Methods: A confidential electronic survey was created to collect information on the availability of colposcopists who encounter patients (female and male) with non-cervical intraepithelial neoplasia and their access to appropriate training. Following approval from the BSCCP executive, this was sent to all BSCCP members who are registered colposcopists.

Results: An e-survey was sent to 1460 BSCCP accredited colposcopists in September 2019. The response rate was $15.2 \%$, with responses from every BSCCP region. 66.3\% stated that their MZIN patients were managed by colposcopists. $17.3 \%$ of respondents reported management in a specialised multizonal clinic. Only $16.4 \%$ answered that a combined service was offered in their hospital, while $83.6 \%$ felt that MZIN should be managed as an integrated service. Conclusions: $46.5 \%$ of colposcopists reported they were satisfied with current service provision. Further investigation of active specialised multizonal clinics could be useful for future planning of MZIN management. These survey results act as an update on current practice and could be used in the development of guidelines.

\section{Abstract \#26: The value of high-resolution anoscopy in the diagnosis of anal cancer precursor lesions among the general population in China}

\section{Yanyun $\mathrm{Li}$}

Department of Obstetrics and Gynaecology Hospital, Fudan University, China

Objective: To evaluate the value of high-resolution anoscopy (HRA) in the diagnosis of anal intraepithelial neoplasia (AIN) among the general population in China.

Methods: A retrospective cohort study was performed, which included 142 patients who underwent HRA at O\&G Hospital of Fudan University from January 2014 to December 2019. With the 
anal biopsy pathology as "gold standard", the diagnostic value of HRA patterns, anal cytology, anal HPV detection were evaluated.

Results: Agreement between HRA and anal pathology was $76.6 \%$ (perianal) and $70.0 \%$ (anal canal), and the strength Kappa were 0.604 (perianal) and 0.455 (canal), respectively. The sensitivity and specificity of HRA in the diagnosis of AIN were $>60 \%$ (Youdan Index $>0.5$ ). The positive predictive value of HRA in canal lesions $(50.8 \%)$ was lower than that in perianal lesions $(68.8 \%)$. HRA diagnosis was more overestimated than underestimated. The anal transformation zone was the predilection site of AIN. The thin acetowhite epithelium was the most common finding in AIN. The dense acetowhite epithelium and vascular patterns were only seen in canal lesions. $88.7 \%$ of AIN patients were HPV (+) and HPV16/18 were the most common subtypes $(\mathrm{P}<0.05)$. The sensitivity and specificity of cytology in predicting AIN were $30.5 \%$ and $95.2 \%$, and that of HPV detection were $88.7 \%$ and $37.7 \%$, respectively. Vulvar intraepithelial lesion was the risk factor of AIN $(\mathrm{P}<0.05)$.

Conclusion: HRA patterns seems reliable in the diagnosis of AIN, which is important for guiding biopsy. However, the diagnosis is more easily overestimated by observers and the canal lesions are more difficult to determine. Cytology and HPV detection are complementary in AIN screening.

\section{Vulval \& Vaginal Disease}

\section{Abstract \#30: Application of 2011 International Federation for Cervical Pathology and Colposcopy Terminology on Detection of Vaginal Intraepithelial Neoplasia}

Hongwei Zhang, Yanyun Li, Qi Zhou, Long Sui

Department of Obstetrics and Gynecology Hospital of Fudan University, China

Objectives: To evaluate the colposcopic accuracy on detection of vaginal intraepithelial neoplasia (VaIN) according to the colposcopic terminology on vagina of 2011 International Federation of Cervical Pathology and Colposcopy (IFCPC).

Method: A total of 467 women who were suspected VaIN and underwent colposcopy at O\&G Hospital of Fudan University from January to December 2018 were included in this retrospective cohort study. The 2011 IFCPC revised terminology on vagina was applied and the agreement between colposcopic diagnosis and vaginal biopsy pathology was analyzed.

Results: Agreement between colposcopy and pathology was $69.16 \%$, and the strength Kappa was $0.437(\mathrm{P}<0.001)$. The agreement was the lowest $(35.71 \%)$ in high-grade VaIN group, which was significantly different from other grade lesion groups $(\mathrm{P}<0.01)$. In grade 1 findings, thin acetowhite epithelium was most frequent $(80.51 \%)$. All the grade 2 findings and vascular patterns were rare. The positive predictive values of micropapillary pattern were $55.98 \%$ for lowgrade VaIN and $5.98 \%$ for high-grade. The specificity of Lugol's nonstaining were $10.92 \%$ for low-grade and $8.30 \%$ for high-grade. There were significant differences in the size of the maximum single lesion and numbers of lesion among different grade lesion groups. The upper third of vagina and posterior wall were the most common for all lesions.

Conclusions: According to 2011 IFCPC terminology, the agreement between colposcopy and vaginal pathology is moderate, and the findings for high-grade ValN may need more definite. The micropapillary pattern can be considered as an alternative low-grade finding, while iodine staining is nonspecific for all lesions. Scattered and spotty lesions suggest low-grade, while large single lesions suggest high-grade.
Abstract \#43: Anal, vulvar and vaginal pathology, in immunosuppressed patients

KristianRubio, Rafael Jose Navarro Avila, Lucia Trillo Rodriguez, Carmen Bellido Bel, Cecilia Villalain Gonzalez, Maria Reyes Oliver Perez

Hospital 12 de Octubre, Spain

Objectives: To evaluate the characteristics and treatment of immunosuppressed patients with HPV related patology in anal, vaginal and vulvar areas.

Methods: Descriptive study on a cohort of 309 patients, 159 with IS treatment, and 150 with HIV diagnosis in follow-up in the Cervical Pathology Unit of our center between 2009 and 2018. The Follow-up was annual according to the Spanish Assosiation of Cervical Pathology and Colposcocopy protocols.

Results: The average age was 47.72 years. Only 14 (4.5\%) had received some type of HPV vaccine. $34.88 \%$ were smokers, $22 \%$ had been diagnosed of an STD and $27.1 \%$ had no steady partner. 107 presented anal pathology of which 74.7 corresponded to ASCUS, $1.9 \%$ to AIN1 and $1.9 \%$ to AIN2. All the patients with ASCUS / AIN1 maintained a wait-and-see approach which showed resolution in all of them. As for the AIN 2, the treatment of choice was the laser in all cases. Vulvar pathology occurred in 39 patients, with HPV being present in $71.5 \%$ of them. Corresponding $84.6 \%$ to VIN $1,2.6 \%$ to VIN $2,7.7 \%$ to VIN 3 and $5.1 \%$ to vulvar carcinoma. 20 patients presented vaginal pathology, of which $100 \%$ presented HPV. $10 \%$ and $35 \%$ had VAIN 2 and VAIN 3 lesions. $66.7 \%$ underwent surgery and $33.3 \%$, with recurrent disease, were treated with brachytherapy. Conclusions: The vulvovaginal and anal pathology rate due to HPV seems higher than in the general population. Special attention should be paid to monitoring this since recurrence rates are especially high at the vaginal area.

\section{Abstract \#56: Prognostic Factors Affecting Outcome of Vulvar Malignancies-A Single Institution Experience}

Deepak Bose, Rema P, Suchetha S, Dhanya Dinesh

Regional Cancer Centre, Trivandrum, India

Background/Aims: To assess the clinical and histopathological features of surgically treated vulvar cancers and to assess overall and disease-free survival of vulvar cancers.

Methods: Retrospective study on women diagnosed with vulvar neoplasm and underwent surgical management from 1st January 2009 to 31st December 2019 in our institution. Clinicopathological features, treatment details, follow-up, recurrence and survival were collected from medical records. Overall and disease-free survival were calculated using Kaplan-Meier method.

Results: A total of 51 patients underwent surgery in our centre for vulvar neoplasms during the study period. Of these $32(62 \%)$ were vulvar cancers of SCC histology stage IA (18.75\%), IB (46.8\%), IIIA $(6.25 \%)$, IIIB $(6.25 \%)$, IIIC $(21.8 \%)$. Most common procedure performed was wide excision vulva $(58.8 \%)$, of which $66 \%$ required inguinofemoral node dissection. Recurrences were seen in 4 of 32 patients $(12.5 \%)$, two each of local and nodal recurrences. Survival was assessed in 27 patients with a median follow-up of 77 months. 3 year overall survival was $76 \%$ and disease-free survival of $75 \%$. Survival rates for tumour size (OS for $<2 \mathrm{~cm}$ vs $>2 \mathrm{~cm}$ : $88 \%$ vs70\%, $\mathrm{p}=0.627$ ), margin status (negative vs positive: OS $88 \%$ vs $50 \%, \mathrm{p}=0.028$ ), nodal status (negative vs positive: OS $81 \%$ vs $73 \%, \mathrm{p}=0.611$ ). 
Conclusion: Our cohort of vulvar cancer patients had an overall survival of $76 \%$, with major prognostic factors being, tumour size, margin and nodal status.

\section{Abstract \#171: Recurrence Patterns and Treatment Outcome of Surgically Treated Vulvar Cancers}

Deepak Bose, Rema P, Siva Ranjith J, Aleyamma Mathew

Regional Cancer Centre Thiruvananthapuram, Kerala, India

Objectives: To assess the recurrence patterns and survival outcomes following surgical management of vulvar cancers.

Methods: A retrospective study was done on patients with vulvar SCC who underwent surgical management from 1January 2009 to 31 December 2019 in our institution. Clinicopathological features, treatment details, follow-up, recurrence and survival were collected from medical records. Overall and disease-free survival were calculated using the Kaplan-Meier method.

Results: Of 33 patients, most common stages were I- $39.4 \%$ (majority of tumours $>4 \mathrm{~cm}$ ) and III- $33.4 \%$. Most common procedure performed was vulvar radical local excision (75.8\%), of which $66 \%$ required inguinofemoral node dissection. Major postoperative complications were wound breakdown $(40 \%)$ and lymphedema $(6.6 \%)$. Recurrences were seen in 13 of 33 patients (39.4\%) - 7 local, 2 groin nodal and 4 distant. Mean time to develop recurrence was 29.5 months. Logistic regression showed adjuvant RT (p-0,004) and stage 3 disease (p-0.042) to be significant prognostic factors for risk of recurrence. With a median follow-up of 75 months, 4 year overall survival was $73.9 \%$ and disease-free survival of $67 \%$.

Conclusions: Recurrences had a significant effect on progression-free survival, without impacting overall survival. Major prognostic factors affecting recurrence were adjuvant radiation therapy and stage of disease. Larger-sampled studies may throw more light onto factors affecting recurrences and survival.

\section{Abstract \#177: Is It Time to Reassign Staging of Pelvic Nodal Involvement in Vulvar Cancers?}

Deepak Bose, Rema P, Siva Ranjith J, Dhanya Dinesh

Regional cancer Centre, Thiruvananthapuram, Kerala, India

Objectives: Pelvic lymph node (PLN) involvement in vulvar cancers has been traditionally considered to be distant metastasis. However, controversy exists regarding the appropriateness of branding them as stage IVB along with other visceral metastasis.

To assess the stage-wise survival outcomes in a retrospective cohort of surgically managed vulvar cancers.

Methods: Women with surgically managed vulvar squamous cell cancers (SCC) over ten years from 2009 to 2019 were included in this retrospective analysis. Overall and disease-free survival rates were calculated using Kaplan-Meier curves.

Results: 33 cases of vulvar SCC underwent surgery from our centre. Stage-wise distribution was stage I- 39.4\%, II- $15.2 \%$, II- 36.4\%, IV with PLN positivity-6\%, IV with distant metastasis- $3 \%$. Median OS for stage IV with PLN was 144 months, which was close to that of stage III (147 months) as compared to that of other distant metastasis 5 months.

Conclusions: Pelvic node involvement in vulvar cancer may be considered as a separate subgroup of distant metastasis with a better prognosis than other hematogenous metastasis. Their survival patterns mirror those of groin node positivity and further analyses could be directed at this aspect of staging and treating vulvar cancer.
Abstract \#178: Adjuvant Radiotherapy in Surgically Treated Vulvar Cancers-A Ten-Year Single Institution Experience

Deepak Bose, Rema P, Suchetha S, Francis V James

Regional cancer Centre, Thiruvananthapuram, Kerala, India

Objectives: Vulvar cancer is an uncommon gynaecological malignancy. Treatment modalities, at times, tend to be non-standardized. We, at a tertiary care cancer centre in India, assessed the outcomes of surgery and adjuvant radiation therapy in our practice spanning over 10 years. To assess the effect of adjuvant radiation therapy in surgically managed vulvar cancers.

Methods: Retrospective study on women who underwent surgical management for vulvar squamous cell cancers (SCC) from $1^{\text {st }}$ January 2009 to $31^{\text {st }}$ December 2019. Clinicopathological features were analysed with respect to adjuvant radiation treatment (RT) details, recurrence patterns and survival rates.

Results: A total of 33 cases of vulvar SCC underwent surgery from our centre. Radiation to vulva was indicated for close or positive margins and groin irradiation for node positive patients. Of the 20 patients in whom RT was indicated, $50 \%$ undertook RT while the other half did not undergo radiotherapy due to personal or logistic reasons. Median PFS was 72 months vs 10 months in favour of patients who underwent adjuvant RT (p-0.002). However, there was no OS benefit. PFS benefit was 65 months for adjuvant RT for positive nodes and 51 months for margin involvement (P-0.006).

Conclusions: Adjuvant radiation therapy, when indicated, has to be administered following surgical management of vulvar cancer owing to its PFS benefit. Further studies may be warranted to tailor dosage of RT to bring in best results.

\section{Abstract \#190: Dysplasia at Postoperative Margins-An Upcoming Prognostic Factor in Vulvar Cancers}

Deepak Bose, Rema P, Suchetha S

Regional Cancer Centre, Thiruvananthapuram, Kerala India

Objectives: Margin positivity is considered to be an important prognostic factor for recurrences in vulvar cancers. There have been conflicting studies regarding the actual significance of close postsurgical margins $(<8 \mathrm{~mm})$ in their risk for recurrences. Dysplasis at margins has now been purported as an important prognostic factor. To assess the recurrence patterns and survival impact of margin involvement in a retrospective cohort of surgically treated vulvar cancers.

Methods: Women underwent surgery for vulvar cancers in our centre from 2009 to 2019. Recurrence patterns and survival rates were assessed with respect to margin status - as under negative, close, positive or dysplasia at margins. Our institutional protocol was to irradiate the vulva postoperatively if there were positive or close margins. Dysplasia was not considered an indication in our practice. Results: Thirty-three patients were surgically treated for vulvar SCC. There were no cases of positive margins. The distribution of negative, close, dysplastic margins was $66.7 \%, 24.2 \%$ and $9.1 \%$; 4-year OS rates of $76.1 \%, 71.4 \%$ and $66.7 \%(\mathrm{p}-0.62)$. Median PFS was 118 months, 56 months and 29 months, respectively. (p-0.10).

Conclusions: Dysplasia at margins, although non-significant for survival benefit, needs to be given importance. Larger sampled studies need to look into this entity and whether adjuvant radiotherapy would provide any treatment benefit in this subset of vulvar cancer patients. 


\section{Abstract \#199: The Outcomes of Ca Vulva Localising To} Clitoris-Single Institutional Study

Pratima Raj, Rupinder Sekhon

Rajeev Gandhi Cancer Institute, New Delhi, India

Objectives: Approximately $4 \%$ of all gynaecological cancers are vulvar carcinoma of which localizing to clitoris makes it a rarity. This study highlights the disease due to its malignant potential and treatment options available. They need aggressive care for better survival. Even with the aggressive therapy the prognosis is very poor in advanced diseases. To evaluate and assess the clinicopathologic characteristics, course of the disease and treatment modalities of patients with vulvar carcinoma localizing to clitoris.

Methods: A retrospective review of patients with carcinoma vulva was evaluated between 2014-2019 at Rajiv Gandhi cancer institute and research center New Delhi.

Results: $20 \%(\mathrm{~N}=14)$ of the 70 patients of vulvar cancer were localizing to clitoris. Mean age of the patients was 63.5 . 7\% were in stage $2,4 \mathrm{~A}, 4 \mathrm{~B}, 3 \mathrm{~A}$, respectively, and $15 \%$ in stage $3 \mathrm{~B}$ and $14 \% 3 \mathrm{C}$ and $43 \%$ in stage 1B. $100 \%$ SCC histology type, of which $28.57 \%$ were well differentiated, $57.15 \%$ were moderately differentiated, $14.28 \%$ were poorly differentiated.78. 57\% underwent radical surgery with bilateral inguinofemoral lymphnode dissection, $7.14 \%$ received primary radition therapy, $14.28 \%$ received palliative therapy. $14.28 \%$ experienced reccurence, 2-year disease-free survival is $80.81 \%$, 2-year over all survival is $58.95 \%$.

Conclusions: Any suspicious lesion needs to be biopsied early for good prognosis of the patient. Management of such cases is a challenge for the surgical oncologist, due to the late presentation, advanced stage at presentation and treatment should be individualised, carried out by a multi-disciplinary team in an experienced cancer centre.

\section{Abstract \#200: Vulvo-vaginal melanoma,10-year experience from a tertiary care center in India}

Aswathy G Nath, Sivarenjith J, Rema P, Suchetha S, Dhanya D, Jagathnath Krishna

Regional Cancer centre, Thiruvanthpuram, India

Background: The ideal management of vulvo vaginal melanoma is still unknown. As the incidences of these cancers are very less, there are limited numbers of studies to formulate an ideal treatment strategy for the same.

Objectives: The purpose of this study is to analyze the outcome of patients diagnosed with vulvo vaginal melanoma.

Methods: Retrospective analysis of case records of patients who underwent treatment for biopsy-proven vulvo vaginal melanoma from January 2006 to January 2016. Overall and disease-free survival was calculated using Kaplan-Meier method and log-rank test for statistical significance. Data was analyzed after grouping patients into 'metastatic group' and 'potentially curative group 'on the basis of status of disease presentation.

Results: Of the 12 patients in the potentially curative group, 7 had vaginal melanoma and 5 had vulval melanoma. Only one patient is alive and disease free among the entire cohort. Median overall survival of these patients was 15 months with a standard deviation of 13.6. Median disease-free survival was 8 months with a standard deviation of 5.9. Among five patients in the metastatic group, lung was the most common site of metastases, followed by liver. Median overall survival was only 11 months with a standard deviation of 3.8.
Conclusion: Vulvo-vaginal melanoma is an aggressive disease with poor prognosis. Major prognostic factors are stage of disease and lymph node status.

\section{Abstract \#285: Our Experience-Ca Vulva, A Single Institutional Study from a Tertiary Cancer Center-India}

Rupinder Sekhon, Pratima

Rajiv Gandhi Cancer Institute and Research Centre, Delhi, India

Objectives: Vulvar carcinoma (VC) is a rare malignancy with an incidence rate of 3-5\% of all gynecological malignancies. The aim of our retrospective study was to analyze the epidemiology, clinical characteristics, and treatment approach of patients with vulvar carcinoma (VC) treated at our cancer Centre.

Methods: We retrieved the information regarding patients' clinical details and treatment given from the case records of all the vulvar cancer patients who were treated during the year 2011-2015 at Rajiv Gandhi Cancer Institute and Research Centre, Delhi.

Results: A total of 85 case records were retrieved for this retrospective analysis. Of these 38 cases defaulted. Median age was 63 years. Squamous cell carcinoma most common histopathology type $(97 \%)$. The stage distribution was FIGO stage IA: $19.14 \%$, stage 1B:34.04\%, stage III A: $4.25 \%$, stage III B-2.12\%, stage IIIC: $25.53 \%$, stage IVB: $8.51 \%$. VIN $3: 6.38 \% .78 .72 \%$ Underwent radical vulvectomy with $\mathrm{B} / \mathrm{L}$ inguinofemoral node dissection, $5.40 \%$ simple vulvectomy, $5.40 \%$ hemivulvectomy, $5.50 \%$ wide local excision, $2.12 \%$ anterior exenteration, $23.40 \%$ received postoperative adjuvant therapy, $8.5 \%$ received palliative therapy. The recurrence rate was $25.54 \%$, most common site vulva $58.33 \%$, followed by inguinal node $33.33 \%$.

Conclusion: The treatment of carcinoma vulva should be individualized with multidisciplinary consultations. The low incidence rates and indolent morbidity of the vulvar disease have led to lack of standardized data regarding the prevalence and treatment of this disease.

\section{Abstract \#289: Vulvar carcinoma Localized To Clitoris-A single institutional study}

Pratima, Rupinder Sekhon

Rajiv Gandhi Cancer Institute and Research Centre, Delhi

Objectives: Approximately $4 \%$ of all gynecological cancers are vulvar carcinoma of which localizing to clitoris makes it a rarity. This study highlights the disease due to its malignant potential and treatment options available. They need aggressive care for better survival. Even with the aggressive therapy the prognosis is very poor in advanced diseases. To evaluate and assess the clinicopathologic characteristics, course of the disease and treatment modalities of patients with vulvar carcinoma localizing to clitoris.

Methods: A retrospective review of patients with carcinoma vulva was evaluated between 2014-2019 at Rajiv Gandhi cancer institute and research center New Delhi.

Results: $20 \%(\mathrm{~N}=14)$ of the 70 patients of vulvar cancer were localized to clitoris. Mean age of the patients was 63.5 years. Out of 14 patients, $7 \%$ were in stage $2,4 \mathrm{~A}, 4 \mathrm{~B}, 3 \mathrm{~A}$, respectively, and $15 \%$ in stage $3 \mathrm{~B}$ and $14 \% 3 \mathrm{C}$ and $43 \%$ in stage $1 \mathrm{~B}$. The histology was squamous cell cancer in all cases, of which $28.57 \%$ were well differentiated, $57.15 \%$ were moderately differentiated, $14.28 \%$ were poorly differentiated. $57 \%$ underwent radical surgery with bilateral inguinofemoral lymph node dissection, $7.14 \%$ received primary radiation therapy, $14.28 \%$ received palliative therapy. $14.28 \%$ 
experienced recurrence, 2-year disease-free survival was $80.81 \%$ and 2 year overall survival was $58.95 \%$.

Conclusion: Any suspicious lesion needs to be biopsied early for the good prognosis of the patient. Management of such cases is a challenge for the surgical oncologist, due to the late presentation, advanced stage at presentation and treatment should be individualized, carried out by a multi-disciplinary team in an experienced cancer Centre.

\section{Abstract \#151: Three Rings Vulvoscopy (TRIV) for Evaluation of Vulvar Disorders-A Pilot Study}

Chingbiaklun Shoute, Amita Suneja

Department of Obstetrics and Gynecology, UCMS \& GTB Hospital, Delhi, India.

Objectives: To test a new approach of Three Rings Vulvoscopy (TRIV) to diagnose vulvar disorders.

Methods: A cross-sectional and observational study was conducted between November 2018 to April 2020. 100 women with vulvar complaints and 100 asymptomatic. All were subjected to TRIV and Conventional vulvoscopy by two different observers. Documentation of specific and non-specific lesions was done in a ring-wise manner on TRIV. Biopsy was taken from pathological lesions found during Conventional vulvoscopy.

Results: The most common symptom among cases was Itching in the vulva $(82 \%)$ followed by burning sensation $(5 \%)$. Vulvar dermatoses depicted non-specific and specific lesions on TRIV in all three rings. VIN depicted only specific lesions mainly in the inner ring (63.64\%) followed by outer ring $(27.27 \%)$. Two more clinical entities were added after vulvoscopy of cases and controls in TRIV i.e., Vulvodynia and Impaired Vulvar skin. TRIV depicted that impaired Vulvar skin has non-specific lesions mostly present in inner ring (92\%). Vulvodynia was diagnosed in the absence of lesions and positive cotton swab test and mostly found in the inner ring. Time taken during TRIV and conventional vulvoscopy was comparable. Inter-rater Kappa agreement between TRIV and conventional vulvoscopy in symptomatic women was found to be a very good agreement.

Conclusions: TRIV is a feasible and systematic approach for interpretation of vulvar lesions. There is a specific pattern of distribution of lesions in the three vulvar rings in different vulvar disorders. TRIV was easier to interpret, more anatomical and systematic. It allows better documentation of vulvoscopy findings.

\section{STDs and HPV}

\section{HIV and HPV}

\section{Abstract \#25: Cervical cancer screening and treatment of cervical intraepithelial neoplasia incorporated in an HIV clinic platform in Pune, India}

Smita Joshi

Prayas, Pune, Maharasthra, India

\begin{abstract}
Aims/Objectives: We are reporting the findings of our cervical cancer screening program among HIV-infected women.

Methods: Screening with VIA, colposcopy, biopsy and treatment of pre-cancer with ablation or excision were provided at extremely affordable rates at Prayas, a non-profit organization, Pune, India. All women screened in the clinic underwent VIA, colposcopy, directed biopsy and ablative treatment during the same visit. Women requiring LLETZ were called at a later date. We also conducted free
\end{abstract}

community outreach clinics supported by donations and provided screening with VIA and treatment of pre-cancer to HIV-infected women. They were screened with VIA followed by immediate ablative treatment whenever eligible. Women not eligible for ablative treatment were called at Prayas for colpsocpy, biopsy and treatment. Results: A total of 2428 HIV-infected women aged 25 to 65 were screened between Nov 2011 and June 2019. The overall VIA positivity was $9.7 \%$ (95\% CI 8.6-11.0). Of the screen positive women, $32.5 \%$ of the women were treated with thermal ablation. Any CIN was diagnosed in $3.5 \%$ of the women $(86 / 2428)$. More than half of the women diagnosed with $\mathrm{CIN}(51 / 86,59.3 \%)$ had CIN 2/3 disease. There were 11 invasive cancers detected corresponding to 4.5 cancers per 1000 women screened.

Conclusions: The high burden of CIN as well as invasive cancer calls for urgent implementation of cervical cancer screening at all NACO ART centres. The detection rates of CIN in this screened population are an underestimate since many of the screen-positive women received ablative treatment without disease confirmation.

\section{Abstract \#37: A comparative study of prevalence of HPV infection, precancerous lesions of Cervix and Vulva in HIV- positive and negative women}

Shainy Peyyala, Reeta Mahey, Shachi Vashist, Naveet Wig, Sandeep Mathur, Pranay Tanwar

Department of Obstetrics and Gynaecology, All India Institute of Medical Sciences, New Delhi, India

Objectives: To detect the prevalence of HPV infection and the presence of abnormal vulvar and cervical lesions in HIV-positive and negative women. Also to see the association of vulvar and cervical HPV infection and comparison of various screening methods among HIV-positive women.

Methods: The present case-control study was conducted at AIIMS, New Delhi from December 2016 to October 2018. HIV-positive women $(n=110)$, aged 30-59 years recruited from Antiretroviral therapy Centre. Age-matched controls $(n=110)$ were recruited from Gynaecology OPD. After a structured interview questionnaire, samples were collected from the vulva and cervix for cytology and hrHPV DNA (Hybrid capture HC2) testing. Vulvoscopy and colposcopy were done using video colposcope. Biopsy was taken from suspicious areas.

Results: The prevalence of abnormal Pap smear was found to be $5.4 \%$ in HIV positive and $1.8 \%$ in HIV negative women. One HIVpositive woman was diagnosed with carcinoma cervix. Prevalence of cervical hrHPV was significantly higher among HIV positive $(20.9 \%)$ compared to HIV negative women $(3.6 \%)(\mathrm{p}<0.001)$. There was a significant association of hrHPV infection with low CD4 counts $<$ 500 cells $/ \mathrm{mm} 3(\mathrm{P}=0.003)$. Prevalence of vulvar hrHPV was more among HIV positive $(15.5 \%)$ compared to HIV negativewomen $(2.3 \%)(\mathrm{p}<0.001)$. Out of cervical hrHPV-positive cases, $43.5 \%$ were concurrently positive for vulvar hrHPV. HPV had highest sensitivity and NPV. Colposcopy had the highest PPV. Pap smear had highest specificity and diagnostic accuracy.

Conclusion: There was a significant difference in the prevalence of both cervical and vulvar hrHPV among HIV-positive and HIV-negative women. The present study proposes combined testing with cervical hrHPV DNA and vulvar hrHPV DNA in HIV-positive women as screening for HPV-related cancers. 
Abstract \#70: Epidemiology of cervical squamous intraepithelial lesions among HIV seropositive rural women

\section{Ganasala Keerthana}

Department of Obs and Gynae RDT Hospital, Bathalapalli, Ananthapur District, India.

Objectives: To study epidemiology and prevalance of cervical squamous intraepithelial lesions among HIV seropositive rural women. The aim of the present study was to perform Pap smear among age group between 21-65 yrs and to assess the prevalance of cervical squamous intraepithelial lesions in HIV seropositive rural women.

Methods: A cross-sectional study was conducted in the Department of Obstetrics and Gynaecology RDT hospital, Bathalapalli. 519 HIV seropositive women who attended care and support center and 519 seronegative women who attend obstetrics and gynaecology outpatient department over a period of 6 months. After $24-48 \mathrm{~h}$ of abstience from vaginal intercourse and usage of tampons and douchings, patient were examined in lithotomy position after wiping out of excess secretions. Two smears were taken one from ectocervix and one from endocervix by Ayre's spatula and by the cervical brush. Smears were spread on glass slide and then fixed in a fixative and results shall be intrepreted accordingly.

Results: Majority of dysplasias among seropositive women who underwent screening in the ae roup of $31-40$ yrs $69.23 \%$ dysplasias were found in seropositive women who married age less than $18 \mathrm{yrs}$. prevalance of study among seropositive women $5 \%$ and seronegative women $1.6 \%$

Conclusion: This study represents a relationship between HIV seropositivity and abnormal Pap smears and stresses the need for periodic pap smear screening in this high-risk population to decrease the global burden of cervical cancer.

\section{Abstract \#105: Assessment of Cervical Pap Smear findings among women with HIV in comparison with other patients attending Tertiary Hospital, Andhra Pradesh}

\section{A Sridevi, B Thirumala Rao, K.L.S Keerthi, K Madhuri \\ Government Medical College, Andhra Pradesh, India.}

Objectives: To assess the Cervical pap smear screening findings in HIV and AIDS patients in comparison with other patients attending Obstetrics and Gynecology Department.

Methods: This prospective study was carried out from September 2019 to January 2020 in the Government General, Hospital Ongole, Andhra Pradesh. We screened 163 sexually active women who were more than 20 years of age and among them 70 were suffering with HIV and AIDS. A detailed history was taken using a predetermined proforma and details were obtained from all women. A sample was taken from the ectocervix and quickly smeared onto a labeled glass slide and fixed with $95 \%$ ethyl alcohol. Laboratory results were reported according to the new Bethesda System for Reporting Cervical Cytology 2014.

Results: Most women were in the age range of 30-50 years in HIV (74.2\%) and non-HIV (56.3\%) Patients. Vaginal discharge was the common complaint ( $24.5 \%$ ), and irregular menstrual cycle (15.9\%) followed by excessive bleeding $(13.2 \%)$ in non-HIV cases. Whereas vaginal discharge noticed in $7.6 \%$ of HIV cases and associated with TB in $2.56 \%$ of cases. inflammatory smears found to be more in HIV Cases $(61 \%)$ in comparison with non-HIV cases, High-grade squamous intraepithelial lesion (HSIL), low-grade squamous intraepithelial lesion (LSIL), and malignant cells were detected in $11.42 \%, 5.71 \%$, and $1.42 \%$, respectively, among HIV cases.
Conclusions: Every woman above the age of 30 years should undergo routine cervical cancer screening, the postmenopausal period. The Pap test has been regarded as the gold standard of cervical screening programs.

\section{Abstract \#145: The Prevalence of abnormal cervical cytology and positivity of Human Papillomavirus DNA among HIV seropositive women}

Archana Mishra, Sunita Malik, Supriti, Harsha Gaikwad, Mausumi Bhardwaj

Department of Obstetrics and Gynaecology, Vardhman Mahavir Medical College and Safdarjung Hospital, New Delhi, India,

Objectives: HIV infection causes impaired cell mediated immunity which in turn is responsible for persistent HPV infection \& increased risk for abnormal PAP Test. The relationship among human immunodeficiency virus, human papillomavirus, and development of Cervical Intraepithelial Neoplasia is undoubtedly complex and incompletely understood. The aim of the current study was to determine the prevalence of abnormal cervical cytology and HPV infection among women with HIV and to compare them with HIV-negative women and the correlation between prevalence of human papillomavirus (HPV) infection and CD4 counts.

Methods: This was a cross-sectional study conducted on 95 HIV seropositive and equal number of seronegative women attending Gynecology OPD. After thorough history and examination specimen was collected from the cervix using cyto-brush for HPV DNA testing, subtyping and cytology.

Results: HPV DNA positivity was significantly higher in seropositive group (18.6\% vs $7.4 \%$ ]. HPV 16 and 18 co-infection was found only in seropositive group. Premalignant condition like AGC, ASCUS, LSIL, HSIL and cell suspicious of malignancy were found significantly higher in Seropositive group. AtCD4 count $<249$, HPV DNA positivity were $53 \%$ and when increased number of CD4 counts in HIV-positive women decrease the percentage of HPV DNA positivity (CD4 counts between 250-499, percentage of HPV DNA positivity were $31 \%$ and at CD4 count $>500$ HPV DNA positivity was $19 \%$ ). Conclusions: It is inferred that the prevalence of abnormal cytology and HPV DNA positivity is higher amongst HIV-positive women, and there was an association between HPV DNA positivity with lower CD4 counts.

\section{Abstract \#186: Epidemiology of Cervical Squamous Intraepithelial Lesions Among HIV Seropositive Rural Women}

Ganasala Keerthana, B. Sumathi

Department of Obs and Gynae RDT Hospital, Bathalapalli, Ananthapur District, India.

Objectives: To study epidemiology and prevalence of cervical squamous intraepithelial lesions among HIV seropositive rural women. The aim of the study was to perform pap smear among age group between 21-65 years and to assess the prevalence of cervical squamous intraepithelial lesions in HIV seropositive rural women.

Methods: A cross-sectional study over a period of 6 months. 519 HIV seropositive women who attended care and support Centre and 519 seronegative women who attend obstetrics and gynecology outpatient department we conduct cross-sectional study on 519 seropositive and 519 seronegative rural women at RDT hospital, after 24-48 h of abstinence from vaginal intercourse and usage of tampons and douching's, patient will be examined in lithotomy position after wiping out of excess secretions. Two smears will be taken one from 
ectocervix and one from endocervix by AYRES spatula and by cervical brush. smears are spread on glass slide and then fixed in a fixative and results shall be interpreted accordingly.

Results: Majority of dysplasia among seropositive women who underwent screening in the age group of 31-40yrs. 69.23\%dysplasias were found in seropositive women who married age less than $18 \mathrm{yrs}$. Prevalence of study among seropositive women $5 \%$ and seronegative women was $1.6 \%$.

Conclusion: This study represents a relationship between HIV seropositivity and abnormal pap smears and stresses the need for periodic pap smear screening in this high-risk population to decrease the global burden of cervical cancer.

\section{Abstract \#205: Comparative Study of Cervical Smear Cytology in HIV-Positive and HIV-Negative Women}

\section{Bhavya AB, Resmy CR}

Department of Obstetrics and Gynecology, Government Medical College, Thrissur, India

Objectives: Screening for cervical cancer is of particular concern to HIV-infected women and adolescents, since the incidence of cervical intraepithelial neoplasia (CIN), as confirmed by colposcopy, is four to five times higher in HIV-positive women compared to the HIV negative community according to various studies. This study attempts to compare the incidence of abnormalities in cervical cytology in HIVpositive women and normal population, risk factors for carcinoma cervix in HIV-positive women and age-matched HIV negative controls, to explore the correlation between cervical abnormalities and CD4 count in HIV-positive women and to determine the relationship with the abnormalities in cervical cytology in HIV-positive women and the duration of ART.

Methods: The study is a cross-sectional comparative study aiming at comparing the incidence of abnormalities in cervical smear cytology of HIV-positive women compared to HIV-negative women in the screening age group of $20-65$. The study was conducted at a tertiary care setting at the Government Medical College Thrissur, under the department of Obstetrics and Gynaecology in association with ART centre functioning under the department of General Medicine and also with the cooperation of the department of Pathology for a period of 1 year (2015-2016).

Sample size was calculated as 65 in each group. Those giving written consent after recruitment were included in the study. Pap smear was taken after proper history taking and examination.

Results: HIV infection was more prevalent in lower socioeconomic status with lower educational qualification as compared to age-matched controls. Delay in the age of marriage and lesser duration of marital life was also noted. BMI was also significantly lesser in HIVpositive group. Definite higher incidence of cervical smear abnormalities has been demonstrated (27.7\% compared to $13.8 \%$ ) in HIVpositive females, even though the result was not statistically significant. The incidence of epithelial cell abnormalities amounted to $7.6 \%$. In the study group only I out of 65 subjects had CD4 count less than 200. A definite relation with duration of ART and cervical abnormalities could not be established.

Conclusions: To achieve the goal of decreasing mortality due to cervical cancer, the HIV-positive population subset should be given more attention since they are studied to have significant higher risk. Steps should be taken to educate regarding periodic pap smear examination along with providing the necessary services and infrastructure.
Abstract \#206: The Association Between Cervical Cancer and HIV in Patients Admitted at Tertiary Care Centre

Naziya Parveen Ansari, Bharti Saxena, R.K Tanwar

Government Medical College, Kota (Rajasthan), India

Objectives: We present a case series of 6 cases of women with cervical cancer with HIV to assess their profile and outcome.

Methods: Retrospective observational study of case records of six cases of cancer cervix with HIV over a period of 9 years. The patients belonged to age group of 30-60 years and were multiparous. The FIGO stage as assessed was IIa to 4 . Most of patients presented with foul smelling discharge and Abnormal vaginal bleeding. The husbands of three of these patients were truck drivers, while other three were labors. All of them belongs to low scoio-economic class None of these patients know their HIV status before they presented to hospital but on testing and on taking history, there spouse found positive and patients herself tested positive for HIV.

Conclusion: Invasive cervical cancer is an AIDS defining condition and because many women in the developing countries present only in the late stages of the disease in absence of organized screening programs, it is often the very first sign of AIDS in these women. There is potential Role of offering HIV testing for selected High-Risk groups of women presenting with cervical neoplastic conditions.

\section{Abstract \#212: Prevalence of Premalignant Cervical Lesions in HIV-Seropositive Women In Rural Area}

Kavya Bahudodda, Sundari N, Jyoti Suchitra

RDT Hospital, Bathalapalli, Anantapur, Andhra Pradesh, India

Objectives: HIV-seropositive women have been found to be at higher risk of HPV infection due to their immune-compromised status and that they are 2 to 12 times more likely to develop cervical precancerous lesions. Aim of this study is to know the prevalence of premalignant lesions in HIV seropositive women in correlation with CD4counts in a rural area.

Design: It is a hospital-based retrospective and observational study. Method: All the HIV-seropositive women attending the RURAL DEVELOPMENT TRUST hospital, Bathalapalli, Andhra Pradesh from January 2017 till January 2020 were included in this study. After taking informed consent, Pap smears were taken from these patients and sent for cytology. The CD4 counts at the time of pap smear reporting were taken into consideration.

Results: There is no association of CD4 counts less than 200 and prevalence of premalignant lesions of cervix in seropositive patients; however, the prevalence of premalignant cervical lesions in seropositive patients increased when compared to normal population. Conclusion: Periodical cytological screening in seropositive patients in rural India is very useful in detection of cervical lesions and thus help in reducing the incidence of cervical cancer.

\section{Abstract \#228: Cervical Cancer Screening Among HIV-Positive Women: A Comparison of Outcome Between VIA and Pap Smear Among Women Accessing Care in South-Eastern Nigeria}

Umeh Uchenna Anthony, Ifebi Okechukwu Chidiebere, Chigbu Chibuike O

Department of Obstetrics and Gynaecology, College of Medicine, University of Nigeria, Ituku-Ozalla, Enugu, Nigeria.

Background: Cervical cancer is the second most common cancer in women especially in developing countries. Human immunodeficiency 
virus (HIV) is a co-factor in the association between human papillomavirus and cervical intraepithelial neoplasia.

Objectives: The study was done to determine the prevalence of premalignant cervical lesion in HIV-positive women accessing care in UNTH, Enugu; compare the sensitivity and specificity of VIA and that of Pap smear, and compare the positive predictive value (PPV) and negative predictive value (NPV) of VIA and Pap smear in detecting cervical premalignant lesions in HIV-positive women.

Methods: This was a cross-sectional comparative study of $438 \mathrm{HIV}$ infected women who attended the PEFAR clinic of the University of Nigeria Teaching Hospital Ituku-Ozalla, in Enugu state. The participants underwent conventional Pap smear, VIA, and Colposcopy. Pap smear and VIA were done by the researcher and the nurses. Colposcopy was then done by the consultant oncologist and was assisted by the researcher.

Results: Six $(1.4 \%)(6 / 438)$ women had unsatisfactory Pap smear results, $13(3.0 \%)$ women had atypical squamous cell of undetermined significance (ASCUS), 42 (9.6\%) women had low-grade squamous intraepithelial lesions (LSIL), $22(5.0 \%)$ of the participants had highgrade squamous intraepithelial lesion (HSIL), and 28 (6.4\%) participants had cervicitis. The study showed that $356(81.3 \%)$ of the participants had normal Pap smear results. 108 (24\%) participants were aceto-white positive, while 330 participants $(75.3 \%)$ were acetowhite negative. VIA had a sensitivity of $60.2 \%$, specificity $96.5 \%$, PPV $51.9 \%$ and NPV $88.8 \%$. For Pap smear, sensitivity was $75.3 \%$, specificity was $88.3 \%$, PPV was $85.4 \%$ and NPV was $93.5 \%$. The prevalence of premalignant cervical lesion as determined by colposcopy in this study was $21.2 \%$

Conclusion: The results from the study showed that VIA is a good screening tool that can be employed in cervical cancer screening especially in low-income countries. VIA is comparable to Pap smear and should be acceptable as a screening tool for HIV-infected women in resource-limited settings.

\section{Abstract \#252: Comparison of Pap Test (Liquid-Based Cytology) and Colposcopy for Detection of Pre-Invasive Lesions of the Cervix in HIV Seropositive Women}

Shalini Singh, Swati Agarwal

Lady Hardinge Medical College and SSKH, Delhi, India

Background: The prevalence of pre-invasive lesions of cervix in HIV seropositive women ranges from $12.5 \%-69 \%$. Although PAP test is the recommended screening method, it has concerns owing to high false-negative cytology in HIV-infected women and high attrition rates.

Aim: To compare the role of routine Colposcopy with Pap test at initial visit for screening of pre-invasive lesions of cervix in HIV seropositive women.

Methods: This was an observational cross-sectional study of 75 HIV seropositive women aged between age 25 to 50 years who were screened for pre-invasive lesion of cervix by PAP test and Colposcopy simultaneously. Colposcopy-directed biopsy was taken if swede score was $>/=5$ and the results were compared with the Histopathology findings.

Results: Out of the 75 women screened abnormal colposcopy was found in 15 women (20\%) out of which $6(40 \%)$ were positive for preinvasive lesion of cervix (4-HSIL and 2-LSIL) on biopsy. Abnormal Pap test were found in 3 women (4\%) and all had abnormal colposcopy and histopathological findings as well. Colposcopy was thus found to superior in detection of pre-invasive lesions of cervix in HIV seropositive women $($ Kappa co-efficient $=0.28$ ).

Conclusion: Depending solely on cytology may miss out on the preinvasive lesions of cervix in HIV seropositive women. Therefore, colposcopy should be the preferred screening modality in these women if facilities permit.

\section{Abstract \#263: Prevalence of Chlamydia and Gonorrhea in HIV Positive and in HIV Negative Outpatients}

Monisha Singh $^{1}$, Nisha Rani Agarwal ${ }^{1}$, Shilpa Agarwal ${ }^{1}$, Jaya Chakravarty $^{2}$, Uma Pandey ${ }^{1}$

${ }^{1}$ Department of Obstetrics and Gynecology, ${ }^{2}$ Department of Medicine, IMS, BHU, Varanasi, India

Objective: To detect prevalence of chlamydia and gonorrhea in HIV positive and HIV negative outpatients, to find correlation of chlamydia and gonorrhea with other sexually transmitted diseases and to assess and compare risk factors associated with STIs.

Methods: A randomized case-control study of duration of 2 years from December 2012 to June 2014, was carried out on patients attending the OPD of obstetrics and gynecology and ART Centre, Sir Sunder Lal Hospital, Institute of Medical Sciences, Banaras Hindu University. The DNA PCR for detection of microorganisms was taken from cervical scraping and was carried out in the department of Medicine. A total of 80 HIV seropositive cases and 20 HIV negative as controls.

Result: Out of 80 HIV-positive cases, 5\% tested positive for Chlamydia, none tested positive for gonorrhea, $8.8 \%$ tested positive for trichomonas vaginalis, $3.8 \%$ tested positive for syphilis, $27.5 \%$ tested positive for HPV. There was significant association of trichomonas with Chlamydia. In our study, CD4 is significantly associated with STD possibility.

Conclusion: Control of the genital chlamydial and gonorrheal infections may have positive implications in the control of HIV transmission and spread in India and vice versa. Prevalence of STDs is higher in HIV-positive population, and low CD4 counts increases the risk of HIV positive to acquire STIs.

\section{1: Training and Education in Colposcopy}

Abstract \#152: Use of Detailed Exam Statistics to Inform Areas for Concentration and Improvement.

Deirdre Lyons, Jennie Byrom, Elaine Radford, Sharon Parisi

BSCCP Certification and Training Committee, BSCCP, Birmingham

Objectives: BSCCP OSCE exam-exit exam that allows colposcopists to enter independent practice.

Aim of the Training Programme:

- Enables students to obtain core

- Develop necessary skills

- Gain professional attributes to enable competency in Colposcopy - OSCE Exit exam

Examination statistics undertaken after every examination to review performance of the exam overall and the individual questions. Previous statistics have shown challenges for candidates in terms of questions related to management of glandular disease. The BSCCP Certification and Training committee (CTC) has reviewed the glandular questions in the question bank and deemed them suitable for examination in terms of management of glandular disease.

Methods: Detailed analysis after each examination has shown candidates are challenged when undertaking written questions and also questions looking at management of glandular disease. The CTC review and update any questions that have shown a consistently high 
failure rate. The data outcomes are publicized at BSCCP Training the Trainers Meetings as part of informing trainers to ensure their trainees are taught the correct management.

Results: Through reviewing detailed examination statistics, the CTC has demonstrated that candidates still have challenges with written questions, especially glandular disease management. To address this the CTC has developed example written questions for trainees and will also hold the workshops at BSCCP Training the Trainers Meetings to help trainers with written question completion and also glandular disease management.

Conclusions: Use of detailed examination statistics can indicate areas of difficulty in practice and allow the CTC to create solutions to improve performance.

\section{Abstract \#216: Colposcopic Evaluation of Postmenopausal Women with Cervical Cytology Showing Inflammation/ Epithelial-Cell Abnormalities}

\section{Snigdha Pathak, G Gandhi, SL Jain, K Agarwal}

Department of Obstetrics and Gynaecology, Maulana Azad Medical College and LNJP Hospital, New Delhi, India

Objectives: To compare abnormal cytology with colposcopy and cervical biopsy in postmenopausal women.

Design: Prospective study.

Methods: The study was performed at a tertiary hospital over one year, and included 60 postmenopausal women with Pap smear showing inflammation/epithelial-cell abnormalities. VIA/VILI was done for all women followed by colposcopy and directed biopsy. When colposcopy was inadequate, vaginal estradiol cream was prescribed.

Results: Of the 60women studied, 23 had pap smear showing 'inflammation'; 18 had low-grade smear (ASCUS, LSIL) \& 19 had highgrade smear (ASC-H, HSIL). On cervical biopsy, high-grade CIN $(\mathrm{CIN} 2+)$ was found in $\mathbf{4 . 3 \%}$ of smears showing 'inflammation', $\mathbf{1 1 . 1 \%}$ of Low-Grade smears \& $\mathbf{7 3 . 7 \%}$ of High-grade smears. Overall sensitivity, specificity \& accuracy of abnormal pap smear (ASCUS \& above) for detecting CIN 2 + was $94.1 \%, \mathbf{5 1 . 2 \%} \& \mathbf{6 3 . 3 \%}$. All participants underwent colposcopy, of which $41.6 \%$ had Type $2 \mathrm{TZ}$ (Transformation Zone) and $21.7 \%$ had Type $3 \mathrm{TZ}$ and satisfactory colposcopic evaluation could be achieved after a course of vaginal estradiol. The sensitivity, specificity \& accuracy of colposcopy (Reid's score 3-8) for detecting CIN2 + was 94.1\%, $\mathbf{7 9 . 1 \% ~ \& ~}$ 83.3\%.

Conclusions: In postmenopausal women, colposcopy was observed to be a significantly better modality than cervical cytology for detection of high-grade CIN. Satisfactory colposcopy could be achieved after a course of vaginal estradiol in type 2,3 TZ.

\section{Abstract \#281: Can portable colposcopes replace standard colposcopes?-A crossover trial}

Jayashree Narajan $^{1}$, Neerja Bhatla ${ }^{1}$, Nimmi Ramanujam ${ }^{3}$, Shachi Vashist $^{1}$, Marlee Krieger ${ }^{3}$

${ }^{1}$ All India Institute of Medical Sciences, New Delhi, India, ${ }^{2}$ Cancer Institute (WIA), Adyar, Chennai, India, ${ }^{3}$ Duke University, NC, USA

Objective: Triage of screen positive women by colposcopy is a major challenge in low-middle-income countries. Portable colposcopes have been developed to overcome the limitations of standard colposcopes. This study aimed to compare the performance of two portable colposcopes with a standard-of-care colposcope in detecting cervical preinvasive lesions.
Design: Crossover randomized study.

Method: Study enrolled women aged 25 to 65 years with abnormal screening result or cervical symptoms. All women underwent colposcopy with two portable colposcopes (Gynocular ${ }^{\circledR}$, Gynius, Sweden and Pocket ${ }^{\circledR}$ transvaginal colposcope, Duke University, NC, USA), followed by standard video colposcope, followed by biopsy. Colposcopy findings were scored by Swede score. The optical characteristics of portable devices were compared to standard colposcopes. The agreement between the portable colposcopes and standard colposcope was calculated. Agreement of Swede score with cervical histology was calculated for each device.

Results: Among 250 subjects, 27 (10.8\%) CIN2 + lesions were detected. The portable devices and standard colposcope had a perfect agreement in Swede score calculation. Swede score with Gynocular and Pocket colposcope was similar to standard colposcope in 247 (98.8\%) and 248 (99.2\%) subjects, respectively (agreement scores 0.9954 and 0.9969 , respectively). At a Swede score cut-off of \&gt;5, all three devices had identical sensitivity, specificity, positive predictive value and negative predictive value of $95.8 \%, 92.1 \%, 57.5 \%$ and $99.5 \%$, respectively. The optical characteristics of portable colposcopes showed significant agreement with the standard colposcope $(\mathrm{P}<0.001)$.

Conclusions: Portable colposcopes performed well compared to the standard colposcope. They can be used in field setting as well as in hospital setup.

\section{Theme 12.0 Miscellaneous and case report}

\section{Miscellaneous}

\section{Abstract \#46: Cervical cancer screening in infertile women}

Kavitha Dhanasekaran, Latha Sriram, Tanuja Joshi, Vipin Kumar, Shalini Singh, Roopa Hariprasad

ICMR-National Institute of Cancer Prevention and Research, Noida, India

Objectives: Infertility among young Indian women is on a high note in recent years. The association of high-risk type HPV infection is being suggested as a major cause for altered fertility among women in recent studies. The objective of this abstract is to emphasize the importance of cervical cancer screening among infertile women.

Methods: ICMR-National Institute of Cancer Prevention and Research functions with the primary mandate of cancer prevention provides free comprehensive cancer screening services as per the operational framework guidelines including cervical cancer screening. All screen test positive women are further evaluated with a colposcopy examination, biopsy (if required) and treated with the ablative method or excisional method whichever is appropriate. A retrospective case review of all women of age $>21$ with a history of primary, secondary infertility and nulliparity who underwent cancer screening from February 2016-December 2019 was done.

Results: Among 435 infertile women underwent cervical cancer screening, 8\% (35/435) were VIA positive. Among all evaluated with a colposcopy examination, 54\% (19/35) had abnormal findings. Among the abnormal colposcopy examinations, 68\% (13/19) had cervical precancerous lesions of which $52 \%$ (10/19) were high-grade lesions; $15 \%$ (3/19) were low-grade lesions and 31\% (6/19) had other conditions like cervicitis, tuberculosis, etc. Of all precancerous lesions biopsied, 31\% (4/13) was CIN3, 23\% (3/13) was CIN2 and $46 \%(6 / 13)$.

Conclusion: Infertile women must not be exempted from screening. All women of age $>21$ attending infertility clinic has to be screened for cervical precancerous lesion along with the infertility workup. 
Abstract \#51: Role of the mode of delivery on the rate of regression of cytologic abnormalities during pregnancy

Ana Belén Bolívar, Rocío Bermejo, Leticia Muñoz, Cristina Almansa, Alvaro Diez, Fátima Martínez

Hospital 12 Octubre, Spain

Objectives: To determine the correlation between the role of delivery and the clearance of cervical cytologic abnormalities.

Methods: Retrospective unicentric study of 116 patients with cytologic abnormalities detected during pregnancy at Hospital 12 de Octubre (Madrid, Spain) between 2009-2018.

Results: The mean age at diagnosis was 29.7 years. $19.8 \%$ patients were smokers, 8 had a history of sexually transmitted infection (STI) and 5 were immunocompromised. $4.3 \%$ had been vaccinated against HPV before diagnosis. The most common cervical cytologic abnormality was ASC-US in $35.3 \%$. High-risk HPV types were detected in 53 patients. $85.3 \%$ of the patients underwent a colposcopy. In 29 cases, a biopsy was performed: LSIL-CIN1 in $17.2 \%$, HSIL-CIN2 in $13.8 \%$ and HSIL-CIN3 in $51.7 \%$ was found. $81.1 \%$ were delivered vaginally while the others by Caesarean section. A total of 90 patients underwent 6-week postpartum re-evaluation: clearance of HPV was $18.3 \%$ and the rate of regression of cytologic abnormalities was $44.4 \%$. No significant difference was found between the two groups (vaginal delivery:76 patients and Caesarean section:14) according to age, race, BMI, smoking, state of immunosuppression, history of STI or parity. Normalization of smears was shown in $45.3 \%$ women who delivered vaginally and in $40.0 \%$ women who underwent Caesarean section $(\mathrm{p}=0.70)$.

Conclusions: The role of the mode of delivery in the rate of regression of cytologic abnormalities is debated. Some reports find a higher rate of regression of cervical dysplasia in association with vaginal delivery compared to Caesarean section, while others do not. Large prospective studies are needed to confirm the hypothetical role of vaginal delivery in the clearance of cytologic abnormalities.

\section{Abstract \#76: Pattern of Cervico-vaginal infections in pregnant women in a tertiary care centre in Bihar}

Hemali Heidi Sinha

Department of Obstetrics and Gynecology, All India Institute of Medical Sciences, Bihar, India

Objectives: Cervico-vaginal infections during pregnancy can threaten the well-being of both mother and fetus. Early detection is important since maternal physiological changes may hamper identification of the infection. The study aimed to evaluate the pattern of cervicovaginal infections among pregnant women attending an antenatal clinic in a tertiary centre in Bihar.

Methods: This 1-year cross-sectional study recruited 200 pregnant women with complaints of vaginal discharge, itching, burning or splash dysuria. Women in both second and third trimesters were included and underwent a speculum examination after consent. The discharge was collected, and a high vaginal swab was also taken from each woman for isolation of pathologic organism. Wet mounts were prepared, gram staining done, Nugent's scoring, and cultures obtained.

Results: Of the 200 women recruited, $16.5 \%$ were found to have Bacterial vaginosis. Iincidence of candidiasis was $24.3 \%$ and trichomoniasis was found in $3 \%$ cases. There were no cases of chlamydia or syphilis, one case of gonorrhea and two cases were HIV seroreactive. Three cases had infection with more than one organism. Group B streptococcus was isolated in one woman. Most patients with infection had intermediate vaginal flora.
Conclusions: Pregnant women with symptoms of vaginal infection should be looked for and treated. Feasibility of routine screening in pregnancy may be explored in the future.

\section{Abstract \#127: HPV infection in male partner in an infertile couple and its correlation with abnormal semen parameters}

Aruna Nigam, Kausar Neyaz, Zeeba S Jairajpuri

Hamdard Institute of Medical Sciences and Research, Jamia Hamdard, Delhi, India

Objectives: The objective of the present study was to determine highrisk HPV infection prevalence in infertile males and correlation between HPV infection and male infertility.

Methods: It was a pilot study done in the infertile couple coming to infertile clinic after obtaining written informed consent. Semen was collected as a routine protocol during investigative workup. The study protocol was approved by the institutional ethical committee. The presence of high-risk HPV DNA in semen samples was investigated by real-time polymerase chain reaction (RT-PCR) using CervSure HPV Detection kit (DSS Imagetech Pvt. Ltd., New Delhi). And the presence of HPV DNA and its correlation with semen parameters was done. $\mathrm{P}<0.05$ was taken as statistically significant.

Results: In this pilot study, 56 semen samples were analysed for HPV DNA in semen samples. The overall rate of HPV positivity was $14.28 \%$ (8/56). Main limitations of our pilot study is relatively small sample size and the lack of a control group of normal fertile men and the lack of follow-up testing to check the clearance or the persistence of HPV DNA in semen after 6-12 months.

Conclusions: HPV prevalence in males is high in the current study and few of the abnormal semen parameters can be correlated with it, but there is a need of large population-based studies to establish a clear role for the same.

\section{Abstract \#191: Why Women with Advanced Carcinoma Cervix Do Not Seek Medical Help Early: A Tertiary Hospital-Based Indian Study}

Anoosha K Ravi, Aastha Raheja, Krishna Agarwal

Department of Obstetrics and Gynaecology, AIIMS, Rishikesh, India

Objectives: The aim of this study was to understand the reason for delayed approach to medical help in women with advanced carcinoma cervix.

Design: It was a cross-sectional study.

Methods: Information obtained from women with carcinoma cervix clinical stage IIb and above with proven histopathological diagnosis was recorded in the predetermined format.

Results: Almost all the subjects were illiterate and were from lower socioeconomic strata and rural areas. None of them knew about the disease and its symptomatology. About $82 \%$ of the patients were symptomatic since 5-10 years and, however, took no medical care during the period and 2/3rd of them did not even reveal their problem to any of the family members. About $79 \%$ of the subjects had never used any contraception, and none had knowledge about its role in preventing infection. Majority of them $(98 \%)$ had no idea about the role of Pap smear in screening carcinoma cervix. All of them said that if they had knowledge about the disease, they would have sought medical help much earlier.

Conclusions: The most important reason for delayed approach in our study was found to be lack of knowledge about the disease. Therefore, till such time that we have national screening program in place, these women who are at high risk of developing carcinoma of cervix should 
be targeted and educated through mass media so that these women could seek medical help in early stages.

\section{Abstract \#236: Impact of Covid 19 Pandemic on the Surgical Care of Patients with Gynecologic Cancers: Experience From a Tertiary Cancer Centre in South India}

Dhanya Dinesh

Regional cancer center, Thiruvananthapuram, Kerala, India

Objectives: To assess the impact of the COVID pandemic on gynecologic oncology surgical practice and postoperative morbidity in a tertiary cancer Centre as compared to the year before the pandemic. Method: Retrospective observational study of all patients undergoing surgery for gynecological malignancies in Regional Cancer Centre, Thiruvananthapuram during the first 6 months of the COVID pandemic between March 1, 2020, and August 31, 2020, compared to patients undergoing surgery during the same period in 2019. Clinical and demographic data including comorbidities, stage of cancer, COVID-19 status, surgical procedure, postoperative stay and morbidity related to surgery were obtained from hospital records.

Results: The total number of surgeries done during the study period in 2020 was 77 compared to 122 in 2019. Three patients had tested positive for COVID 19 and had their surgeries one month after testing negative. $2.6 \%$ of patients had minimally invasive surgeries compared to $5.7 \%$ in 2019 . Complex procedures comprised $23.4 \%$ in 2020 compared to $20.5 \%$ in 2019 . A higher percentage of patients with ovarian cancer underwent neoadjuvant chemotherapy in $2020(93.3 \%$ vs $80.6 \%$ ). Intraoperative blood transfusions were given for $14.3 \%$ vs 4.9\%. Post-operative ventilation was used for $5.2 \%$ vs $3.3 \%$. Unplanned readmissions were $5.2 \%$ vs $1.6 \%$. There was one postoperative mortality in 2020 who was a patient who tested positive in the preoperative period and had a bowel perforation and sepsis.

Conclusions: Even with all the restraints imposed by the pandemic, we continued to do complex surgeries including minimally invasive procedures. Although the postoperative complications were more in number in 2020 , a statistically significant difference was seen only in case of intraoperative blood transfusions and prolonged ileus.

\section{Abstract \#244: Feasibility of ERAS Protocols in Management of Patients with Gynaecological Cancers During COVID19 Pandemic}

Rema P, Suchetha S, Mallika Balakrishnan, Aswathi Nath Sivarenjith

Regional cancer center, Trivandrum, Kerala

Objective: Enhanced Recovery after Surgery (ERAS) is a multimodal perioperative care pathway designed to achieve early recovery after surgical procedures. This study aimed to analyze the feasibility of ERAS in the era of pandemic and to find its effect on the postoperative outcome of patients undergoing surgery for gynecological cancer during the COVID pandemic.

Methods: This observational study was done on patients who underwent gynecological cancer surgery during COVID pandemic in our institution. Data was collected including patient demographics, nature of surgery, adherence to each of the components of ERAS programme and outcomes. Post-operative complications were graded according to the Clavien-Dindo classification.

Results: A total of 112 patients were included in the study period from June to October 2020.65 patients had cancer ovary, 41 cancer endometrium, 5 cancer cervix and 1 cancer vulva. In the pre-operative component of ERAS protocols, $82 \%$ patients received pre-surgery counselling, $97 \%$ received thromboembolic prophylaxis, $94 \%$ received carbohydrate loading and none of the patients received mechanical bowel preparation. $8 \%$ received blood components during and after surgery. In the post-operative phase on Day 1, 62\% patients had urinary catheter removed, $88 \%$ received normal diet and $92 \%$ had early ambulation. The complication rate was $26 \%$ (29 patients), but majority $79 \%$ had grade 1 and 2 complications. There was one postoperative mortality due to sepsis. The mean hospital stay was 6.6 days.

Conclusions: The study confirms the feasibility and benefits of following ERAS pathway in enhancing patient recovery during COVID pandemic.

\section{Abstract \#267: Cervical Cancer Incidence and Mortality in South} East Asia: Evidence From Globocan 2020

Smita Asthana, Satyendra Singh Yadav, Shalini Singh

ICMR-National Institute of Cancer Prevention and Research, Noida, Uttar Pradesh, India

Objectives: Cervical cancer is one of the leading malignancies among females in South East Asian region (SEAR) as defined by WHO. This study aims to examine the cervical cancer burden in SEAR using recently released Globocan 2020 estimates.

Study Design: Ecological.

Methods: Age- and country-wise incidence and mortality estimates for cervical cancer were obtained for 11 SEAR countries using data available from Globocan 2020. Age-specific disease burden was analyzed using incidence, mortality and MI ratio (mortality/incidence). Data on human development index (HDI) was extracted from United Nations Development Programme report. Bivariate correlation analysis was done for HDI in relation to Incidence, Mortality and MI ratio separately.

Results: In SEAR cervical cancer, estimates for the year 2020 were 190,874 cases and 116,015 deaths. Age-standardized incidence rate (ASIR) was 18.1/100,000 and Age-standardized mortality rate (ASMR) was $11.1 / 100,000$. India was the leading country with 123,907 cases and 77,348 deaths. ASIR was highest among 50-55 years age group whereas ASMR was maximum in 55-59 years age group. Association between HDI and MI ratio was negative with significant correlation $(r=-0.664, p=0.026)$. Association between ASIR and HDI as well as ASMR and HDI though negative but was found to be non-significant.

Conclusion: To reduce cervical cancer burden in Southeast Asia region, main challenges are lack of cancer awareness, lack of systematic screening, late stage at disease presentation and poor treatment facilities. Mortality is higher in older age group. There was an inverse relationship between HDI and cervical cancer incidence and mortality in the SEAR.

\section{Abstract \#308: Change in practice in cervical cancer screening during COVID 19 Pandemic}

Chandrima Ray, Ranajit Mandal, Puja Chatterjee

Department of Gynaecological Oncology, Chittaranjan National Cancer Institute, Kolkata

Objective: This study aims to analyse changes in practice for cervical cancer screening in rural districts of West Bengal before and after the COVID-19 pandemic led to disruption of preventive healthcare services.

Design: Retrospective cross-sectional study.

Method: Data from March 2019-March 2020 and April 2020-March 2021 was retrospectively analysed from women aged 30-59 years 
who underwent screening in rural districts of West Bengal as part of Integrated Programme on Non-Communicable Diseases Prevention project, which has been implemented at our institute since March 2017. After onset of nationwide lockdown due to the pandemic, screening services were withheld for 2 months. From June 2020, HPV testing kits (QIAGEN) were distributed and collected by our team of health workers, while maintaining all precautions. Women who tested positive underwent colposcopy with or without treatment (by thermal ablation or LEEP) at their nearby primary health centre by our clinicians.

Results: From April 2019-March 2020, 6748 women were screened using either HPV DNA testing (Hybrid Capture 2) or VIA. Cervical samples for $\mathrm{HC} 2$ testing were collected from 5581 women, either by provider $(57.28 \%, \mathrm{n}=3197)$ or self-sampling $(42.71 \%, \mathrm{n}=2384)$. VIA was done in 1167 women, 25 were positive $(2.14 \%)$. From the 200 women $(3.58 \%)$ who were HC2 test positive, colposcopy was performed on 113 women $(56.5 \%)$. Treatment was done in 87 (76.99\%) women. Thermocoagulation was the most common method used $(77.02 \%)$ followed by LEEP $(21.8 \%)$. From June 2020-March 2021, 5875 women were screened using only self-sampling method for HPV testing, of which 268 were $\mathrm{HC} 2$ positive $(4.56 \%)$, of whom $183(68.28 \%)$ underwent colposcopy. Treatment was done in 149 women $(81.4 \%)$, thermocoagulation being the most common $(81.87 \%)$ procedure followed by LEEP $(16.77 \%)$.

Conclusions: HPV self-sampling can be promoted during COVID-19 pandemic as it curtails travel and reduces human contact.

\section{Abstract \#314: Are infertile women more prone to abnormal cervical cytology than fertile women?}

Sarita Singh, Priyanka Naik, Geetika Khanna

VMMC and Safdarjung Hospital, Delhi, India

Objectives: To compare the frequency of abnormal cervical cytology in infertile and fertile women. We tested the hypothesis that women with infertility have a higher risk of abnormal cervical cytology as compared to fertile women.

Design: It was a prospective cross-sectional study conducted in the Department of Obstetrics \& Gynecology at a tertiary teaching hospital, Delhi. Conventional cervical cytology was used for the study as it is easily available and cost-effective.

Methods: Women attending Gynecology OPD, and infertility clinic were recruited after taking informed consent. A total of 370 women were included in the study (185 in each group). The Pap smear was done and reported as per Bethesda classification 2020. The participants details were entered in the study proforma. Chi-square and unpaired student t-test were used for data analysis.

Results: The infertile women were younger $(\mathrm{p}<0.05)$. The age of first intercourse and sexual contact was earlier infertile women. Both groups had awareness about RTI and STI. Women with infertility had higher frequency of post-coital bleeding $(p=0.006)$. In fertile women, incidence of ectopy was higher as compared to infertile women (0.002). The ASCUS frequency was comparable and no CIN was reported in both groups.

Conclusions: Infertility does not increase the risk of abnormal cervical cytology. The study did not evaluated incidence of HPV. We recommend that infertile women should also undergo cervical cancer screening as frequently as fertile women.

\section{Case reports}

\section{Abstract \#35: A case report of carcinoma vulva}

\section{BKN Sudha}

RDT Hospital, Anantapur, India

Case Report: Mrs. R, 38 years multiparous woman, belongs to low socioeconomic status, known HIV positive, on ART from 18 years, came to RDT Hospital, Anantapur, with history of progressive lesions over vulva from 6 months, treated as Herpes infection outside, no contributing family or personal history. On examination she has good performance status. Right inguinal lymph node enlarged $3 \times 3 \mathrm{~cm}$, hard, nontender and fixed. Ulcerated growth over the whole right labia majora and minora extending laterally $4 \mathrm{~cm}$ from midline, vertically $2 \mathrm{~cm}$ below clitoris, up to fouchette. Pink in colour, irregular elevated margins, minimal serosanguinous discharge from the growth. Base indurated, firm in consistency, irregular, tender, friable, does not bleed on touch. Clitoris and urethra are not involved. Right lateral vaginal wall lower $1 / 3$ and rectovaginal septum lower $1 / 3$ feels indurated.

Diagnosis: 38 years multi-parous woman HIV positive on ART, vulvar cancer, Stage $4 \mathrm{~A}$. Biopsy from the lesion showed squamous cell carcinoma, grade 3. As she was Stage 4A she was referred for radiotherapy.

Discussion: This case is Type 1 cancer as she is young, associated HIV, sexually transmitted diseases. Vulvar malignancies account for $3-5 \%$ of all cancers of genital tract. $90 \%$ are squamous cell carcinomas. Lymphatic spread of cancer is to inguinal and femoral lymph nodes.

\section{Abstract \#39: Vulvar Müllerianosis: case report and literature review}

Leticia Muñoz Hernando, Lucía Trillo Rodriguez, Beatriz Risco Montemayor, Jara Montero Olmeda, Leticia Muñoz Hernando, Kristian Rubio Lorenzo

Hospital Universitario 12 de Octubre, Spain

Objectives: Description of a case of vulvar müllerianosis, a rare entity, and literature review.

Case report: A 44-year-old woman without any history of gynecologic nor pelvic procedures, came to our clinic with a nodular and painful lesion in external genitalia, next to clitoris that appeared three years ago. She referred an increase in the size and pain in the days before menstruation, as well purulent and blood-stained spontaneous drainage. In the examination, we observed a $1.5 \mathrm{~cm}$ nodule in the superior section of right labia minora, next to the clitoral hood, with two red patches in its surface and without apparent drainage hole. Due to the characteristics of the lesion, its growth and the progressive aggravation of the associated symptoms we decided to perform a biopsy. While we waited for the result we began with a continuous regimen of combined estrogen-progestin oral contraceptives. After a month of treatment, the patient's symptomatology got worse. The result of the anatomical pathology analysis showed "scamous mucosa which stroma and glands without atypia and no evidence of endometrial stroma, suggesting Müllerianosis as diagnosis". We surgically removed the lesion, including its capsule, confirming that it did not affect the clitoris.

Conclusions: Müllerianosis is the term used to describe those lesions formed by müllerian glands in ectopic localizations. It differs from endometriosis in the presence of ciliated glandular elements and no endometrial stroma. The recommended treatment is surgical removal of the lesion with free margins. 
Abstract \#40: Expression of HPV-associated disease in an immunocompromised woman: multifocal and multicentre HSIL lesions with genital leishmaniasis

Alejandro OlloquiEscalona, ReyesOliver Pérez, Cristina AlmansaGonzález, Alvaro Diez Alvarez, Ana Belén Bolivar de Miguel

Hospital Universitario 12 de Octubre, Spain

Objectives: To show how HPV infection expresses in an immunocompromised woman, often as a multicentric and multifocal disease with difficulties on treatment and follow-up, and with a high rate of recurrence and disease progression.

Methods: This is a case report of a 41-year-old HIV-positive woman non-receiving antiretroviral therapy. Viral load 221,386 copies $/ \mathrm{mL}$, CD4 count 1 cell/microL (2\%), associating visceral and cutaneal leishmaniasis. She complained of genital ulcers and vulvar pruritus from several months ago.

Results: Clinical examination showed multifocal and multicentric extensive lesions with abnormal colposcopic findings grade 2, in vagina, vulva, perianal region, anus and cervix (photography will be provided). All the biopsies showed HSIL and leishmaniasis. Treatment consisted in conization, excision of lesions in vagina, vulva, anus and perianal region and laser vaporization of the small lesions. Recurrence of HSIL in all locations occurred six months after treatment, and one lesion in vulva was diagnosed as a squamous cell carcinoma. She presented also an adenopathic conglomerate in inguinal region, which biopsy showed metastases of squamous cell carcinoma. Staging showed disseminated lymph node metastases.

Conclusion: This case shows how HPV infection can affect immunocompromised women, resulting in a more extensive and aggressive disease, with more recurrences, often needing multiple treatments and with a rapid progression to invasive carcinoma. In immunocompromised women it is important to optimize the immunitary system, giving antiretroviral therapy in HIV-positive women, and to consider examination of all the genital area, as it is frequent to find multifocal and multicentric HPV-associated disease.

\section{Abstract \#60: An unusual presentation of Cervical Intraepithelial Neoplasia 3}

\section{Seetha Panicker, Jeeva S, Chitra Bhat TV}

PSG Institute of Medical Sciences \& Research, Coimbatore, Tamilnadu, India

Objectives: Screening and diagnosing pre-invasive and invasive lesions in a postmenopausal woman is a difficult problem.

Case report: Mrs. X, 61 yrs, a known hypertensive, Ischemic Heart disease patient, postmenopausal since 20 yrs, Nullipara, had Colposcopic examination. The colposcopic findings were warty proliferative growth on cervix extending to right fornix and a dense acetowhite epithelium at 6 ' $O$ clock position. The findings were suggestive of invasive carcinoma cervix. But, cervical biopsy report came as papillary carcinoma in situ (CIN III). Subsequent LLETZ and vaginal biopsy were also reported as non-invasive papillary carcinoma in situ (CIN III)

Conclusion: Warty proliferative growth suggesting invasive cancer may sometimes be only CIN III. This is a challenging situation as it may be misclassified in post-menopausal women and sometimes may be difficult to exclude invasive carcinoma.
Abstract \#77: Tuberculosis of uterine cervix presenting with recurrent leucorrhea in guise of carcinoma of cervix

Priyanka Singh

Datta Meghe Institute of Medical Sciences, Wardha, Maharashtra

Objective: Leucorrhea is a common gynecologic symptom and affects quality of life of women when it becomes recurrent. Tuberculosis as cause of abnormal-looking Cervix or growth on cervix is not commonly thought of. We present a rare case who presented with recurrent leucorrhea having growth on cervix clinically appearing like cervical malignancy but on histopathology was found to be tuberculosis of cervix.

Results: Tests for HIV antibody and VDRL infections were carried out and were negative, and the chest $\mathrm{x}$-ray was normal. Pelvic ultrasonography showed a regular-sized uterus and normal bilateral adnexa lacking any masses or collection. Sputum, urine and cervical secretions were negative for acid-fast bacilli and culture was negative. The erythrocyte sedimentation rate (ESR) at $2 \mathrm{~h}$ was $85 \mathrm{~mm} / \mathrm{hour}$ and Mantoux test was positive. Patient was not willing for further investigations such as CBNAAT and TB PCR for diagnosis due to financial constraints. She was then registered for DOTS (directly observed and started on anti-tubercular drugs as advised byrespiratory physician. She has completed intensive phase of Anti tubercular treatment and is currently in follow-up with remarkable improvement of symptoms.

Conclusions: Tuberculosis should be ruled out in cases of recurrent leucorrhoea or unhealthy non-malignant cervix and in low-resource settings. Diagnosis can be based on clinical and histopathology findings as seen in our case for timely initiation of treatment.

\section{Abstract \#156: A Rare Case Report of Synchronous Cervical Cancer with Cancer Ovary}

S. Spattnaik, J Parija, BL Nayak, MR Mohapatra, J J Mohapatra, A, K Padhy, S Samantray, R Das, N Panda, S Panda

\section{AHPGIC, Cuttack, India}

Case Report: A 45-year-old female presents with pain abdomen and post-menopausal bleeding for last 5 months, menopause attained, nulliparous. On examination a suprapubic mass of 20 weeks size, firm, and restricted mobility. On per speculum examination a growth was seen arising from the endocervix. On per vaginal examination uterine height could not be elicited, lower pole of mass felt, restricted mobility, fornices were full. Her routine investigations were normal. Her CA 125 was 393 IU/ml, CEA- 2.9 IU/ML Inhibin-4.5 PG/ML, her smear showed presence of metaplastic cell with nuclear atypia. Her Diagnostic hysteroscopy, endometrial biopsy and endocervical curettage was done. Intraoperatively the growth was seen filling the lower endometrial and endocervical cavity. Histopathology was suggestive of adenocarcinoma with squamous metaplasia. Her CECT showed presence of bulky uterus expanded cavity with a large necrotic mass extending down to endocervix, left side parametrial involvement seen with a globular solid necrotic deposit left para uterine position. Adnexal region a mass of $145 \times 83 \mathrm{~mm}$. Similar deposits in the para colonic gutter in right iliac fossa. Tiny nodules in lung field. Moderate ascites was present.

Discussion: The endocervical growth stained negative for vimentin, and p16 + ve confirming endocervical growth. The plasma CEA was raised to $2.9 \mathrm{IU} / \mathrm{ml}$, favoring the endocervical growth. The pap smear shows atypia is also suggestive of cervical cancer. One of the important features which rules out metastasis is that for endometrial cancer without myo-invasion and absent LVSI to have a para uterine and ovarian involvement, i.e., in favor of synchronous cervical 
cancer. The HPE of ovary showed unilateral well-differentiated endometroid without involvement of capsule, LVSI -ve, elevated CA125 in postmenopausal age group. The HPE of ovarian carcinoma is as that of a primary. Moreover, ovarian cancer stains + ve for pax8. Thus, asynchronous ovarian carcinoma with cervical cancer is a challenging entity and needs careful evaluation for accurate diagnosis and prognostication.

\section{Abstract \#207: Cervical Cavernous Hemangioma: An Enigma in A Postmenopausal Woman}

\section{Sonam Sharma}

Department of Pathology, Kalpana Chawla Government Medical College, Karnal, Haryana, India

Objective: Cervical hemangioma is an unusual female genital tract pathology, which is seldom encountered. It is a great masquerader of cervical malignancy and can lead to a diagnostic dilemma as well as unnecessary aggressive interventions. One such rare case in a postmenopausal female is being described to create awareness among the clinicians about this enigmatic entity.

Case Report: A 63-year-old female presented with an intermittent postmenopausal bleeding of 6 months duration. Per speculum examination revealed a spongy and bulky cervix, which bled on touch. Bimanual pelvic and rectal examination was unremarkable. Provisionally, a cervical malignancy was suspected. The patient underwent total abdominal hysterectomy with bilateral salpingooophorectomy. On histopathological evaluation, grossly, the uterus with cervix measured $8 \mathrm{~cm} \times 5 \mathrm{~cm} \times 3 \mathrm{~cm}$. On cut section, cervix had hyperemic portions and visible livid areas. Microscopically, multiple thin-walled vascular channels of varying sizes having erythrocytes within the lumen were seen in the cervical stroma. No features suggestive of malignancy were present. Based on these findings, a final diagnosis of cervical benign vascular tumor-cavernous hemangioma was made.

Discussion: Cervical hemangiomas are very uncommon with about 60 cases reported worldwide. They mainly involve the women in their reproductive age and are rarely seen in the postmenopausal period. Majority are asymptomatic and are detected incidentally; nevertheless, they can cause severe obstetric and gynecological complications which can be life-threatening. They can also be misdiagnosed as malignancy especially in postmenopausal women as in the present case. Therefore, a high index of clinical suspicion and meticulous histopathological examination is the mainstay for establishing the diagnosis.

Conclusion: Cervical hemangioma, although a rare entity, should always be considered as a differential diagnosis for postmenopausal bleeding.

\section{Abstract \#208: Recurrent Squamous Cell Cervical Cancer Presenting with Cutaneous Metastases}

Pratima Raj, Rupinder Sekhon

Department of Gynae Oncology, RGCIRC, India

Objective: Squamous cell carcinoma of cervix frequently metastasizes to the pelvic lymph nodes and a distant metastasis is unusual which often noted in liver, lung, and bone. Cutaneous metastasis is an infrequent presentation. The treatment is typically palliative and has a worse prognosis.

Case report: A 58-year-old woman was diagnosed cervical squamous cell carcinoma stage 3 . She was treated with concurrent chemoradiation and brachytherapy. On routine follow-up 12th year, she developed recurrence in bilateral inguinal lymph nodes and received 4 cycles chemotherapy. During treatment, she noticed many nodular lesions on her anterior lower abdominal wall. Biopsy from the skin showed metastatic PDC. PET scan showed no evidence of metabolically active lesion anywhere in the body. She received two cycles of palliative chemotherapy. She developed hemiparesis after completion of second cycle chemotherapy. NCCT head was suggestive of large area of infarction involving left parietal and temporal lobes. She is due for third cycle.

Discussion: The commonest site of cutaneous metastasis is anterior abdominal wall. The interval between cutaneous metastasis and initial diagnosis 0-19 years. Any skin lesion in these patients should be biopsied. Till date, there is no clear guidelines for the management due to its rarity.

Conclusion: Metastases to skin is a rare manifestation of cervical carcinoma with poor prognosis necessitating early recognition and to include skin examination during follow-up. The treatment is palliative and includes radiotherapy, chemotherapy, wide local excision a combination or either of these.

\section{Abstract \#222: Post-hysterectomy Vaginal Cuff Cancer Secondary to CIN: A Case Report}

Zohra Fathima, Vidya A Thobbi

Al ameen Medical College Hospital, Bijapur, Karnataka

Objectives: The incidence of vaginal intraepithelial neoplasia and vaginal cancer is very low, at 0.2 to 0.3 and 0.42 per 10,000 . Conclusive evidence is there to prove that cancer cervix occurs secondary to HPV infection Women over 60y of age who has CIN lesions are at increased risk and should be followed up closely.

Case report: A 58y old P4L4, who had come with complaints of intermittent vaginal bleeding since past 6 months. On investigation, she had multiple fibroids. Also, Pap smear was taken as routine screening procedure. It revealed low-grade squamous intraepithelial lesion of the cervix. No HPV testing was done due to lack of resources. She underwent total abdominal hysterectomy. The HPE confirmed diagnosis of localized low-grade CIN 1. After 4 months she presented with complaints of WDPV. Vaginal cytology test was done, and it showed dyskaryosis, with grade 2 high-grade VAIN. Patient opted for radiotherapy to avoid second surgery. Two months after radiotherapy, follow-up colposcopy and biopsies showed no tumor tissue or recurrence, and she is doing well.

Discussion: We all know that HPV which can cause ca cervix can also cause other cancers like ca vagina. This case shows the importance of screening for HPV as a primary method of screening also as follow-up method.

Conclusion: This case proves the importance of HPV testing as a screening method and postoperatively for follow-up.

\section{Abstract \#225: Limb Amputation: An Unacceptable Sequela of Radiation Therapy For Cervical Cancer}

Reetu Hooda, Hemant More, Anjali Gupta

Department of Obstetrics and Gynecology, Postgraduate Institute of Medical Sciences Rohtak, Haryana, India.

Objectives: To highlight a rare and distressing complication (Radiation induced occlusive artery disease) of radiation therapy for cervical cancer.

Case Report: A 44-year-old $\mathrm{P}_{4} \mathrm{~L}_{2}$ with advanced cervical cancer who had undergone radiation therapy as primary treatment. Although on regular follow-up, she failed to report the subtle numbness she was experiencing in the lower limb 3-yrs postradiotherapy. 
Discussion: Radiation arteritis with gangrene of lower limb had already ensued when the patient sought medical advice for sudden onset acute pain in the limb, 5-yrs post-treatment. Amputation was inevitable by then.

Conclusion: Radiation arteritis must be kept in mind as a sequela of radiotherapy. Early recognition of warning signs (like numbness) might prove instrumental in salvaging a limb.

\section{Abstract \#226: Two rare cases of vaginal STUMPs-A Surgical Challenge}

Deepak Bose, Suchetha S, Siva Ranjith J

Department of Gynecological Oncology and Surgical Oncology, Regional Cancer Centre Thiruvananthapuram, India

Objectives: STUMPs (or Smooth muscle Tumors of Uncertain Malignant Potential) are smooth muscle tumors that are not unequivocally benign or malignant on histopathological basis. Their evaluation depends on degree of cellular atypia, mitotic figures, and tumor cell necrosis. Although smooth muscle tumors are commonest variety of the rare entity of vaginal mesenchymal tumors, vaginal STUMPs have not been reported yet in literature. The treatment of choice for vaginal smooth muscle tumors is surgical excision. However, this is usually a surgical challenge owing to the proximity of these of tumors to sensitive organs.

Case report: We report two cases of vaginal STUMPs that are presumably the first in literature. These cases involved vaginal masses that grew paravaginal and were diagnosed after excision to be STUMPs. Both patients were premenopausal parous women in their mid-forties. Both presented with feelings of pelvic pressure progressing to perineal swellings over a period of 5-20 years. MR imaging showed a large $\mathrm{T} 2$ hyperintense lesions arising from vaginal wall and growing paravaginal to ischiorectal and ischio-anal fossa, compressing lower urethra and anal sphincters. Core biopsy depicted a spindle cell neoplasm. They underwent surgery from our centre on December 2019 and January 2020. The former underwent a wide excision via abdominal and perineal incisions, whereas the latter had a paravaginal excision through a perineal approach alone. Excisions could be completed without urinary, bowel or sphincter injuries. Owing to the histological diagnosis of STUMP, they have been under follow-up and are disease-free.

\section{Abstract \#234: Vaginal Myomectomy of an Endocervical Submucous Myoma}

Shravani Mohite

MGM Women's and Children's Hospital, Kalamboli, Navi Mumbai

Myoma (also called fibroids) are benign tumors composed partly of muscle tissue. Cervical myomas are smooth, benign tumors in the cervix and are closer to organs such as bladder, ureter, and rectum. Ultrasound (TAS \& TVS) and MRI are the effective diagnostic tools for assessment of myoma and plan the management accordingly. Myomectomy or hysterectomy is indicated in cases of persistent bleeding with infertility despite medical therapy. This case came with c/o menorrhagia with postcoital bleeding with anemia with USG suggestive of $4.5 \times 4.2 \mathrm{~cm}$ submucosal fibroid in endocervix. Vaginal myomectomy is the route of choice in pedunculated submucosal myomas along with accurate preoperative assessment of myoma's size, localization, and vascularization. In this case, myoma impacted in cervix and its prolapse on pressure with opportunistic tracing of its stalk was the indication for vaginal myomectomy. To reduce recurrence of myoma, reduce post-surgical endometriosis, improve dysmenorrhea and dyspareunia, improve hematocrit in anemic patient, a GnRH analogue (leuprolide acetate) is recommended.

\section{Abstract \#257: Immunohistochemistry as an ancillary aid in differentiating Endometrial from Cervical Adenocarcinoma}

Charu Sharma $^{1}$, VikarnVishwajeet ${ }^{2}$, Shashank Shekhar ${ }^{1}$, Payal Patwa $^{3}$, Pratibha Singh ${ }^{1}$

${ }^{1}$ Department of Obstetrics \&Gynecology, ${ }^{2}$ Department of Pathology AIIMS Jodhpur, ${ }^{3}$ AIIMS, Delhi

Objective: Cervical cancer is the most common gynecological malignancy in India. At times, differentiating endometrial adenocarcinoma from cervical adenocarcinoma becomes challenging only on histopathology. In such cases, immunohistochemistry (IHC) brings us out of the maze.

Case report: A 45-year-old female presented with heavy menstrual bleeding for past 6 months. Examination revealed a midline 18-week size, hard suprapubic lump. Cervix was apparently normal looking. Ultrasonography suggested an increased endometrial thickness of $19.5 \mathrm{~mm}$ along with AGC-NOS (atypical glandular cell—not otherwise specified) on pap smear. Endometrial Biopsy was suggestive of adenocarcinoma. On MRI Pelvis, a polypoid mass involving lower body and cervical stroma extending superiorly into endometrial cavity and with $>50 \%$ myometrial invasion was observed. Internal iliac and paraaortic lymph nodes were enlarged. Clinical diagnosis of endometrial cancer stage III $\mathrm{C}_{2}$ was made. Patient was planned for radical hysterectomy. Luckily, before surgery we got IHC done which revealed the cells immune reactive for p16 and CEA (carcino-embryonic antigen) and negative for Vimentin, ER and p40 confirming endocervical adenocarcinoma, and the treatment plan was revisited. Patient was started on radical chemo-radiation therapy followed by ICBT.

Discussion: Selected antibody panel on IHC must be tailored according to the specific diagnostic context. Endometrioid adenocarcinomas are usually strongly Vimentin positive and CEA negative, while endocervical Carcinomas are only rarely and very focally Vimentin and CEA positive. Similar findings have been supported by the literature.

Conclusion: Our case highlights the fact that IHC acts as an ancillary aid in the management of such difficult cases where unknowingly we could have landed up in a wrong decision of operating a higher stage cervical carcinoma.

\section{Abstract \#262: Rhabdomyosarcoma Cervix-Unusual tumor in young girl}

Shuchi Agrawal, Seema Mehrotra, Vandana Solanki

Department of Obstetrics \& Gynaecology, KGMU, Lucknow

Objective: To present unusual case of Rhabdomyosarcoma Cervix in young girl.

Case Report: A 15-year-old girl presented with complaint of discharge, irregular bleeding per vaginum and pain in abdomen for last 1 year. Examination revealed a non-tender suprapubic mass of 14-16 weeks size, with irregular margin and restricted mobility. On local examination dirty blood mixed discharge seen. On per rectal examination, there was an irregular mass of around $7 \times 6 \mathrm{~cm}$ felt to be arising from cervix, rectal mucosa free, parametrium free. CT scan showed bulky uterus with large heterogeneously enhancing SOL in lower uterine segment involving cervix with multiple necrotic areas of around $9 \times 8$ with no metastasis elsewhere. On examination under anesthesia, vagina was filled with necrotic friable growth arising from postlip of cervix and biopsy revealed an embryonal Rhabdomyosarcoma (botryoid variant). Patient received IRS-IV protocol neo- 
adjuvant chemotherapy followed by excision of cervical growth. Patient has received 28 cycles of chemotherapy and is planned for further radiotherapy. Presently patient is relieved of her symptoms.

Conclusion: Cervical RMS is a rare tumor occurring in young females and should be included in the differential diagnosis of a patient presenting with recurrent vaginal discharge and irregular bleeding per vaginum refractory to treatment.

\section{Abstract \#273: Atypical Presentation of Cervical Cancer in Postmenopausal Woman-A Case Report}

Swayamprava Behera, Puspanjali Khuntia

SCB Medical College, Cuttack, India.

Objective: Early detection and diagnosis of cervical cancer with atypical presentation.

Case Report: A 62-year-old woman, P4L4 presented with symptoms of weight loss, anorexia, night sweats, and cough. She had no history of postmenopausal bleeding. she was admitted in the hospital. After few days she developed abdominal pain, vomiting and brown vaginal discharge. Computed tomography scan showed a pyometra extending to the umbilicus, with an intrauterine contraceptive device noted inside. After retaking thorough history, she told copper- T 380 A was inserted 25 years back. The patient was not given information regarding insertion of cu-T. The pyometra was drained and the cu-T was removed. Cervical cancer was suspected during the pyometra drainage and biopsies taken. The patient sepsis improved after pyometra drainage but histology subsequently confirmed stage1B squamous cell cervical carcinoma. She was referred to oncologist for a radical hysterectomy.

Conclusion: Postmenopausal women should be routinely screened for cervical cancer. Cervical cancer can present with nonspecific symptoms. Good history, thorough examination to be done to diagnose cervical cancer. There is a correlation between intra-uterine contraceptive device and carcinoma cervix. Thus, women with intra-uterine contraceptive devices need to be informed (verbally and preferably in written) for appropriate follow-up.

\section{Abstract \#286: Bowen's disease of vulva \\ Pratima Raj, Rupinder Sekhon \\ Rajiv Gandhi Cancer Institute and Research Centre, Delhi, India}

Background: Bowen's disease is a type of squamous cell carcinoma in situ. It can develop on any part of the skin surfaces or mucous membranes, but vulvar lesions are rare. The diagnosis is based on the histopathological examination. If untreated, $10 \%-12 \%$ can progress to invasive carcinoma. Treatment modality can be either wide local excision, simple vulvectomy, electrocoagulation and $\mathrm{CO}_{2}$ laser ablation or medical therapies such as 5-fluorouracil or 5\% imiquimod, systemic cidofivir, and photodynamic therapy. Surgery is the standard treatment, due to the multifocal nature of disease. Photodynamic therapy is being evaluated as an alternative for cosmetic purposes.

Case Report: A 41-year-old hypertensive, obese, diabetic lady presented with 9 months history of itching and white plaque lesion over vulva. She was undergoing treatment for primary infertility. On physical examination $2.5 \times 2.5 \mathrm{cms}$ discolored plaque-like lesion on lower one third of right labia majora extending onto the left labia majora. Per speculum / vaginal and rectal examination was normal. Biopsy of the vulvar lesion showed Bowenoid type squamous cell carcinoma in situ. Simple vulvectomy was done taking $2 \mathrm{~cm}$ margin of healthy tissue. Final histopathology confirmed the previous biopsy report of Bowen disease of vulva with typical Bowen cells. Three years postoperation, the patient is undergoing routine regular followup with no signs of recurrence.

Conclusion: The clinical Characteristics of vulvar Bowen's disease are mostly pigmented patches over vulva associated with itching. Diagnosis is based on histopathological uniqueness of Bowen cells and surgery is the preferred treatment.

\section{Abstract \#288: Recurrent Cervix Uteri Cancer Presenting with Cutaneous Metastases-A Case Report}

Rupinder Sekhon, Pratima

Rajiv Gandhi Cancer Institute and Research Centre, Delhi, India

Background: Squamous cell carcinoma of cervix frequently metastasizes to the pelvic lymph nodes and a distant metastasis is unusual which often noted in liver, lung, and bone. Cutaneous metastasis is an infrequent presentation. The commonest site of cutaneous metastasis is anterior abdominal wall. The interval between cutaneous metastasis and initial diagnosis varies from $0-19$ years. The treatment is typically palliative and has a worse prognosis.

Case report: A 58-year-old woman was diagnosed cervical squamous cell carcinoma stage 3 . she was treated with concurrent chemoradiation and brachytherapy. On routine follow-up 12th year, she developed recurrence in bilateral inguinal lymphnodes and received 4 cycles chemotherapy. During the course of treatment, she noticed many nodular lesions on her anterior lower abdominal wall. Biopsy from the skin showed metastatic PDC. PET scan showed no evidence of metabolically active lesion anywhere in the body. She recieved two cycles of palliative chemotherapy. She developed hemipareseis after completion of second cycle chemotherapy. NCCT head was suggestive of large area of infarction involving left parietal and temporal lobes. She is due for third cycle.

Conclusion: Metastases to skin is a rare manifestation of cervical carcinoma with poor prognosis necessitating early recognition and to include skin examination during follow-up. Any skin lesion in these patients should be biopsied. Till date, there is no clear guidelines for the management due to its rarity. The treatment is palliative and includes radiotherapy, chemotherapy, wide local excision a combination or either of these.

\section{Abstract \#293: Misoprostol for Pyometra Drainage in Cervical Cancer: A Case Report}

Lakshmi, Poonam Sachdeva, Y.M. Mala, Shakun Tyagi

Department of Obstetrics and Gynecology, Maulana Azad Medical College \& associated Lok Nayak hospital, New Delhi

Objective: To assess misoprostol as a sole agent for pyometra drainage in cervical cancer with distorted cervical anatomy.

Case report: A 45-year female, diagnosed as carcinoma cervix (large cell keratinizing squamous cell) stage IIIb was referred to us for further management.

On examination cervix was replaced by an irregular growth measuring $5 \times 6 \mathrm{~cm}$ involving both lips and upper vagina. Cervical os couldn't be made out. Uterus was enlarged to $10-12$ weeks size, soft in consistency, bilateral parametrium was thickened just short of pelvic wall. CEMRI abdomen \& pelvis showed a growth of $4.6 \times 5.8 \mathrm{~cm}$ involving endocervical region and extending into upper1/3rd of vagina. Pyometra measuring $4.2 \times 2.9 \mathrm{~cm}$ was present. Patient was planned for pyometra drainage followed by concurrent chemoradiation (CCRT). Considering the distorted cervical anatomy with large growth 200ug misoprostol was inserted pervaginally. Patient gave history of soakage of pad and ultrasonography revealed a decreased volume of pyometra $(2 \times 1.5 \mathrm{~cm})$. Same dose of 
misoprostol was repeated after $24 \mathrm{~h}$. Ultrasonography performed after $48 \mathrm{~h}$ showed negligible amount of intra-uterine fluid.

Discussion: Misoprostol may be used per vaginum to facilitate cervical dilatation and drainage of pyometra. The dose may be repeated based on ultrasonography findings.

Conclusion: Pyometra drainage is challenging surgical procedure in distorted cervical anatomy \& friable growth. There is a high risk of false passage creation by cervical dilators, causing injury to adjacent organs like bladder \& rectum. In such cases misoprostol may be tried before contemplating a cervical dilation to reduce major complications.

\section{Abstract \#305: Title: 'Double trouble'-Cytologic diagnosis of concurrent dual infections on cervical cytology}

\section{Parikshaa Gupta, Nalini Gupta}

Postgraduate Institute of Medical education and Research, Chandigarh, India.

Objective: Diagnosis of polymicrobial infections on cervical cytology has been sparsely documented in the literature. We present 2 cases where the diagnosis of dual infection could be rendered on cervical Papanicolaou smears.

\section{Case Reports:}

Case 1: A 32-year-old woman presented with a history of frothy discharge per vaginum associated with itching. Per speculum examination revealed inflamed and erythematous cervix. A cervical sample was collected. The Papanicolaou-stained SurePath ${ }^{\mathrm{TM}}$ cervical preparation showed predominance of superficial and intermediate squamous epithelial cells with inflammatory changes. There were numerous flagellated organisms conforming to the morphology of Trichomonas vaginalis in the smear. In addition, a few squamous epithelial cells with multinucleation, nuclear molding, chromatin margination and prominent dense eosinophilic intranuclear inclusions surrounded by a clear halo were seen, consistent with Herpes simplex virus (HSV) infection. Case 2: A 27-year-old, retrovirus-positive woman presented with a history of white discharge per vaginum. Per speculum examination revealed cervical erosions. A cervical sample was collected. Sur$e$ Path $^{\mathrm{TM}}$ cervical preparation showed many scattered koilocytes indicating human papillomavirus infection. Additionally, there were scattered metaplastic cells showing multinucleation with nuclear molding, chromatin margination and dense eosinophilic intranuclear inclusions surrounded by a clear halo, indicating HSV infection.

Conclusion: The index cases highlight the diagnostic utility of inexpensive and non-invasive cervical cytology in the detecting such polymicrobial infections, besides being the most successful screening test for cervical cancer.

\section{Abstract \#306: Atypical Presentation of Cervical Cancer in Postmenopausal Woman}

\section{Swayamprava Behera}

Department of Obstetrics and Gynecology, SCB Medical College, Cuttack

Background: Globally cervical cancer kills millions of women every year. Cervical cancer screening routinely in post-menopausal women decrease the development of cervical cancer.

Objectives: Early detection and diagnosis of cervical cancer with atypical presentation.

Case Report: A 62-year-old woman, P4L4 presented with symptoms of weight loss, anorexia, night sweats, and cough. She had no history of postmenopausal bleeding. She was admitted in the hospital. After few days, she developed abdominal pain, vomiting and brown vaginal discharge. Then she had computed tomography scan which showed a pyometra extending to the umbilicus, with an intrauterine contraceptive device noted inside. After retaking thorough history, she told copper- T 380 A was inserted 25 years back. The patient was not given information regarding insertion of cu-T. The pyometra was drained and the cu-T was removed. Cervical cancer was suspected during the pyometra drainage and biopsies taken. The patient sepsis improved after pyometra drainage but histology subsequently confirmed stage1B squamous cell cervical carcinoma. She was referred to oncologist for a radical hysterectomy.

Discussion: There is a correlation between intra-uterine contraceptive device and carcinoma cervix. Thus, women with intra-uterine contraceptive devices need to be informed (verbally and preferably in written) for appropriate follow-up.

Conclusion: Postmenopausal women should be routinely screened for cervical cancer. Cervical cancer can present with nonspecific symptoms. Good history, thorough examination to be done to prevent the cervical cancer.

\section{Abstract \#307: Gastric type adenocarcinoma of cervix with bilateral ovarian masses: a case report}

Atul Sharma, Rajesh Kumari, Sarita Kumari, Anjali R, Romey Rai, Sandeep Mathur, Neerja Bhatla

Department of Obstetrics and Gynecology, All India Institute of Medical Sciences, New Delhi

Objective: Gastric type, endocervical adenocarcinoma is a rare subtype of cervical carcinoma. It is HPV-independent and very aggressive. It can present in unconventional ways and usual symptoms of cancer cervix may be absent.

Case report: We report a case of gastric type, endocervical adenocarcinoma in a 62-year old patient presenting as low backache and bilateral ovarian masses. On evaluation, the clinic-radiologic diagnosis of stage IIIc1r cancer cervix with involvement of bilateral parametria was made and patient was planned for laparotomy to further evaluate the ovarian masses. Laparotomy revealed disseminated intraperitoneal disease with omental involvement in addition to ovarian masses. Final histopathology of intraperitoneal disease and ovarian masses was reported as metastatic endocervical adenocarcinoma, gastric type, stage IVB. The patient was then started on palliative chemotherapy. Conclusion: Gastric type adenocarcinomas of cervix are known for their aggressiveness and show unusual metastatic patterns compared to usual type endocervical adenocarcinoma. This case highlights the unusual presentation, aggressive behavior and poor prognosis associated with gastric type cervical adenocarcinoma. In addition, it also brings attention to the non-conventional route of metastasis in carcinoma cervix.

\section{Abstract \#319: Role of IHC for accurate diagnosis of CIN: A Case report}

Bharati Abhyankar, Tanvee Kulkarni Patel

Purva hospital, Kolhapur, Maharashtra

Cytology is still very important method of primary screening as well as follow-up screening.

The present case highlights that even in limited-resource settings as that of the author, proper screening, treatment and follow-up and advanced methods like IHC can be used for accurate diagnosis.

Case Report: Fifty-two-year-old, post-menopausal lady presented with complaint of pain in abdomen on and off and insisted on cervical cancer screening. In 2017 Pap smear report was negative for 
intraepithelial lesion or malignancy (NILM), with severe inflammation and reactive cellular changes. In 2020 repeat smear was ASC-H cannot exclude HSIL. HPV DNA test was negative, colposcopy was atrophic cervicitis. LEEP was performed and histopathology showed CIN1/IMMATURE METAPLASIA consistent with complete excision. IHC was also consistent with CIN1 and repeat cytology after one year i.e., in 2021 was NILM.

Conclusion: In post-menopausal women colposcopy does not help in diagnosing the premalignant lesion. Cytology still has place in proper diagnosis. Even if HPV is negative but cytology is positive, one must proceed and manage the case with the help of colposcopy doing LEEP OR LEETZ. Metaplasia is confusing finding, IHC helps in differentiating between CIN lesion and metaplasia.

\section{Abstract \#324: Port Site Metastasis After Robotic-Hysterectomy for Uterine Cervical Cancer-A Case Report}

Amita Naithani, Rupinder Sekhon, Pratima

Department of Gynaecologic Oncology, Rajiv Gandhi Cancer Institute and Research Centre, New Delhi

Background: Squamous cell carcinoma of cervix commonly metastasizes to the lymph nodes of the pelvis. Incidence of port site metastasis from carcinoma cervix is $0.1-2 \%$. The treatment is usually palliative. Here, we report the case of a patient who suffered port site metastasis after robotic hysterectomy for stage IB uterine cervical cancer.

Case report: A 43-year-old premenopausal woman was diagnosed cervical squamous cell carcinoma. She underwent robotic type 3 radical hysterectomy with bilateral salpingoophorectomy with bilateral pelvic lymphadenectomy. Postop histopathology showed poorly diiferentiated carcinoma (PDC), stage $1 \mathrm{~b}$, with no lymphovascular space invasion but deep stromal invasion. She received adjuvant EBRT followed by brachytherapy. 20 months after finishing the therapy, single nodular lesion appeared on her abdomen at port site. PET CT showed metabolically active cutaneous soft tissue enhancing lesion in supraumbilical region $2.6 \times 2.3 \mathrm{cms}$, and left axillary lymphnodes $3.3 \times 2.6 \mathrm{cms}$. FNAC from the lesions suggestive of metastatic poorly differentiated carcinoma. She received 5 cycles of chemotherapy and is due for 6th cycle.

Conclusion: Port site metastasis of cervical cancer is infrequent. Guidelines concerning treatment are not available in literature due to uncommonness of cases. The management must be individualized and mostly depends upon the extent of disease. Biopsy is obligatory to prove. Close follow-up including examination and imaging with special consideration to incisional, port, or drain sites to detect recurrence. Treatment of port site recurrences remains palliative chemotherapy, radiotherapy, or wide surgical excision.

\subsection{Abstracts from MBBS students}

\section{MBBS \#1: Aparajita Kale, Asmita Suri, Shambhavi Varma}

\section{Lady Hardinge Medical College, New Delhi, India}

A Web-based Survey on Awareness about Cervical Cancer and its Vaccine.

Introduction: Cervical cancer is one of the most common cancers in the world, caused by human papillomavirus (HPV), a sexually transmitted pathogen. Cervical cancer vaccine offers protection against HPV infection; however, its uptake is poor in the target age group.
Objective: To assess awareness amongst college students about cervical cancer, its screening, and prevention.

Design: Online Google Forms-based survey $(\mathrm{N}=24$ multiple choice questions).

Methods: From 7th April 2021, the survey was circulated using social media platforms. 110 responses were recorded through a Google spreadsheet as of 14th April 2021.

Result: Mean age of the respondents was 21 years (range: 17 to 25 years); majority were aware about the existence of cervical cancer $(94.5 \%)$, the causative agent $(80.9 \%)$, effective screening methods $(85.5 \%)$ and that it is a preventable cancer $(68.2 \%)$. A substantial proportion of them were unaware of the existence $(41.8 \%)$ or types of vaccines $(81.8 \%)$. Nearly $50 \%$ had a misconception that cervical cancer is a genetic disease. There was a lack of knowledge regarding age for regular screening (84.6\%), best age for vaccination $(55.5 \%)$ and requirement of post-vaccination screening (40\%). Only $10 \%$ of them had taken the HPV vaccine.

Conclusion: The study highlights limited knowledge of college students regarding cervical cancer prevention. There is an urgent need for creating awareness in target age groups, which is possible through social media and education programs to eliminate misconceptions.

\section{MBBS \#2: 'CervPRThaM' (सर्वप्रथम)—An App as a Complete One-Step Solution for Cervical Cancer.}

Ishan Gupta, 2nd Professional MBBS Student, AIIMS, New Delhi

Background: India alone accounts for $1 / 5$ th of the total cervical cancer burden of the world. We envisage the development of a National Portal by the Government of India with a mobile-based application for prevention \& treatment of cervical cancer in India. This is an attempt to increase acceptance of HPV vaccination \& screening tests among women.

Methods: A mobile-based application by the name of 'CervPRThaM'-Cervical Cancer Prevention Repository, Therapeutics and Management (सर्वप्रथम) whose meaning in Hindi is - 'First \& Foremost' highlighting how our daughters \& mothers should be put first and foremost in our fight against cancer is to be developed. The app will have cervical cancer awareness videos, will send reminders to the users whenever their vaccination or screening is due. For screening, medical personnel will upload pictures of VIA and/or Pap Smear tests \& results of HPV tests on the app. Gynaecologists and/or pathologists will review these. The next steps to be taken as recommended for management will be recorded in the app.

Results: The database formation will help in preparation of future strategies of prevention and treatment of cervical cancer. The mobile application will provide all information of a patient at one place. We will roll out the app on a larger population and see how well it is taken up by the people.

Conclusions: This app will have comprehensive information of all the preventive strategies for a woman at one place and will act as a one-step solution for prevention of cervical cancer. 
Title - 'CerwPRThaM' (सर्वप्रथम) - An App as a Complete One-Step Solution for Cervical Cancer

Ishan Gupta, $2^{\text {nd }}$ Professional MBBS Student, AIIMS, New Delhi

\section{Abstract}

Background: India alone accounts for $1 / 5^{\text {th }}$ of the total cervical cancer burden of the world. We envisage the development of a National Portal by the Govemment of India with a mobile-based application for prevention \& treatment of cervical cancer in India. This is an attempt to increase acceptance of HPV vaccination \& screening tests among women.

Methods: A mobile-based application by the name of 'CervPRThaM' - Cervical Cancer Prevention Repository, Therapeutics and Management (सर्वप्रथम) whose meaning in Hindi is - 'First \& Foremost' highlighting how our daughters \& mothers should be put first and foremost in our fight against cancer is to be developed. The app will have cervical cancer awareness videos, will send reminders to the users whenever their vaccination or screening is due. For screening medical persomel will upload pictures of VIA and/or Pap Smear tests \& results of HPV tests on the app. Gynaecologists and/or pathologists will review these. The next steps to be taken as recommended for management will be recorded in the app.

Results: The database formation will help in preparation of future strategies of prevention and treatment of cervical cancer. The mobile application will provide all information of a patient at one place. We will roll out the app on a larger population and see how well it is taken up by the people.

Conchusions: This app will have comprehensive information of all the preventive strategies for a woman at one place and will act as a One-Step Solution for prevention of cervical cancer.

Publisher's Note Springer Nature remains neutral with regard to jurisdictional claims in published maps and institutional affiliations. 